Geomicrobiology of the Ocean Crust: the Phylogenetic Diversity, Abundance, and Distribution of Microbial Communities Inhabiting Basalt and Implications for Rock Alteration Processes

By

CARA M. SANTELLI

B.S. Geology, University of Wisconsin, Madison, 2000

Submitted in partial fulfillment of the requirements for the degree of

Doctor of Philosophy

\begin{abstract}
at the
MASSACHUSETTS INSTITUTE OF TECHNOLOGY

and the

WOODS HOLE OCEANOGRAPHIC INSTITUTION

June 2007
\end{abstract}

(C)Cara M. Santelli, 2007

All rights reserved.

The author hereby grants to WHOI permission to reproduce and to distribute paper and electronic copies of this thesis in whole or in part.

Signature of the author

Jont Program in Oceanography, Massachusetts Institute of Technology and Woods Hole Oceanographic Institution March 23, 2007

Certified by

Wolfgang Bach, Thesis Co-Supervisor

Certified by

Katrina J. Edwards, Thesis Co-Supervisor

Accepted by

Timothy I. Eglinton, Chair, Joint Committee for Chemical Oceanography, Woods Hole Oceanographic Institution 


\title{
Geomicrobiology of the Ocean Crust: the Phylogenetic Diversity, Abundance, and Distribution of Microbial Communities Inhabiting Basalt and Implications for Rock Alteration Processes
}

By

\author{
Cara M. Santelli \\ Submitted in partial fulfillment of the requirements for the degree of \\ Doctor of Philosophy \\ to the \\ MIT/WHOI JOINT PROGRAM IN OCEANOGRAPHY \\ on March 9, 2007
}

\begin{abstract}
Basaltic ocean crust has the potential to host one of the largest endolithic communities on Earth. This portion of the biosphere, however, remains largely unexplored. In this study, we utilize molecular biological, microscopic, and geochemical tools to gain a better understanding of the geomicrobiology of the ocean crust. Specifically, we examine the phylogenetic diversity of microorganisms inhabiting basaltic lavas, the activities and abundances of these microorganisms, the spatial extent of the biosphere, and the potential effect that microbial activity has on the geochemistry of the ocean crust and overlying water column.

Our study demonstrates that young, fresh volcanic lavas near mid-ocean ridges host an incredibly diverse and dense population of microorganisms dominated by Bacteria, quite distinct from the microbial communities found in surrounding deep seawater and hydrothermal vents. Furthermore, these communities may contribute to the elemental cycling of $\mathrm{Fe}, \mathrm{S}, \mathrm{Mn}, \mathrm{N}$, and $\mathrm{C}$ in this environment. The inability to definitively identify microorganisms in drill-cores of old ( $>15 \mathrm{Ma}$ ) ocean crust, however, implies that these once prolific communities may become scarce as the crust ages and moves further away from the ridge axis. Finally, we provide evidence suggesting that these communities are fueled by oxidative alteration reactions occurring in the basaltic crust.
\end{abstract}

Thesis Co-Advisor: Wolfgang Bach

Adjunct Scientist, Woods Hole Oceanographic Institution Professor, University of Bremen, Germany

Thesis Co-advisor: Katrina J. Edwards Associate Professor, University of Southern California 


\section{Acknowledments}

Funding for this project came from: RIDGE 2000 Grant \#OCE-0241791 awarded to Katrina J. Edwards and Wolfgang Bach, the NASA Astrobiology Institute - Cycle 3 CAN-02-OSS-02 awarded to Katrina J. Edwards, USSSP ODP Post-cruise funding Grant \#TX A\&M F001724 awarded to Cara M. Santelli and Katrina J. Edwards, and a Schlanger Ocean Drilling Fellowship awarded to Cara M. Santelli.

I thank my advisors, Katrina Edwards and Wolfgang Bach, for their endless patience and support. You have taught me to think critically and to see "the big picture". Environmental science means very little if you can't put it into context. I also thank you for providing me with numerous opportunities to pursue so many different aspects of our field. I was never limited, either scientifically, academically or socially, as a graduate student. My thesis committee (Karen Casciotti, Fred Frey, and David Smith) has been incredibly accommodating and has given me complete freedom to carry out my thesis project as my advisors and I see fit...even the last minute additions and changes. Their experience, insight, and feedback are very much appreciated.

This project would not have been possible without the support of so many incredible individuals. For every chapter of my thesis, I had the opportunity to collaborate with amazingly talented and inspiring scientists...Virginia Edgcomb, Brandy Toner, Craig Moyer, and Neil Banerjee have all been an integral part of this project. I have learned more from you than I can possibly thank you for. Everyone in the Edwards' lab (Dan Rogers, Sheri Simmons, Erin Banning, Peter Canovas, Olivier Rouxel, Ouafae Rafie, Clara Chan, Ed Leadbetter) has contributed scientifically and emotionally to this project...you all made my grad school experience so enjoyable and memorable! I am also indebted to the Mitch Sogin Lab at the Marine Biological Laboratories for giving me access to all of your resources thus making my research (and life) so much easier. I especially thank Hilary Morrison, David Mark Welch, Julie Huber, Rich Fox, and Leslie for being incredibly patient and helpful with all of the sequencing and phylogenetic analyses for this project. Louie Kerr at the MBL has also been great in teaching me and helping me with all of the microscopy performed in this thesis. Matthew Marcus and Sirine Fakra have been instrumental in teaching me the fine art of XAS/XRD and Beamline 10.3.2. Dan Fornari, Maurice Tivey, and Hans Schouten allowed me to participate in their research cruise AT11-7 and were incredibly accommodating...not too many people would graciously sacrifice their beautiful lava drips in the way of microbiology! I also appreciate all of the great work by the captain and crew of the $R V$ Atlantis and DSV Alvin.

Thank you to the department of Marine Chemistry and Geochemistry for giving me a chance and not giving up on me. Meg Tivey has been so supportive of me from the day I started...I especially enjoy your lab/office chats! I also appreciate all the help that Margaret Sulanowska and Brian Schroeder have given me along the way. I need to thank 
so many people in the Geology and Geophysics Department for sharing their lab equipment with me and because you generally made my graduate school adventure a much richer experience - Greg Hirth, Henry Dick, Glenn Gaetani, Susan Humphris, Stan Hart, Karen Hanghoj, and Peter Clift deserve a special thanks! Eric Webb and Stefen Sievert from the Biology Department also gave me access to their lab equipment, which was very much appreciated. I also can't forget Nan Trowbridge for helping maintain morale.

The Joint Program would not be the amazing graduate program that it is without the dedication of the Academic Programs Office. I especially thank Julia Westwater and Marsha Gomes for making my life so much easier and for just generally taking care of all of us students. Thanks to all the JP students who have shared this experience with me...especially all you of you in the Marine Chemistry program and the Geology program. My friendships with so many great people have always been appreciated and will never be forgotten - Anna Michel, Paul Craddock, Kristy Dahl, Rachel Wisniewski, Helen White, Mike Jakuba, Dave Lund, Mea Cook, Heidi Fuchs, Rose Came, Jessica Warren, Clare Williams, Rachel Stanley, Brian DeMartin, Matt Jackson, Nick Drenzek, Carlos Moffat, Mike Braun, Adam Soule, and so many more. Jeff Standish, Rhea Workman, Linda Kalnejais, Margaret Boettcher, Brandy Toner, Dylan Millet, and Leo Toner-Millet have been the greatest housemates ever! I cherish your friendships and all the fantastic times we've had together.

Jeff, thank you for everything you have given me over the past several years - you have helped me through this experience in so many ways. I know it wasn't easy to just stand there and hold my hand without offering your experience, but I'm extremely grateful for your support and understanding and the perks that came along with it (making me dinner, taking me skiing or surfing, going for walks, listening to me rant and rave, cleaning the apartment, listening to my favorite music of the day, or just making me laugh)! We've had so many fantastic adventures together and I look forward to many more (although you could lay easy on the quadruple black diamond runs with excessive cliffs!).

I could not have started or finished my graduate school career without the never-ending love and support from my family. Mom and Dad - you two have always supported me in every decision (OK, well almost every decision) and truly made me believe that I could do anything and become anyone I wanted with a little blood, sweat, and tears...ahh, the great mid-western mentality! You two are my greatest inspiration in life. Thank you for always believing in me. Patrick - we've been through a lot in the past couple years, and I am grateful for how strong our friendship has become in reflection of that. And to my grandparents, aunts, uncles, cousins (and again my parents and brother)...I know that you have no idea why I would possibly want to stay in school this long (especially for something that will not make me rich or famous), but I very much appreciate your support, interest, humor, and guidance in so many aspects of my life!

I dedicate this dissertation to my parents, because they deserve it! 


\section{TABLE OF CONTENTS}

ABSTRACT .................................................................................................... 3

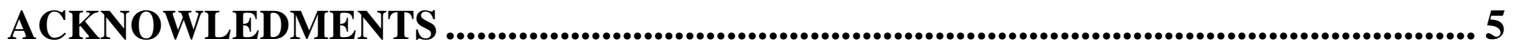

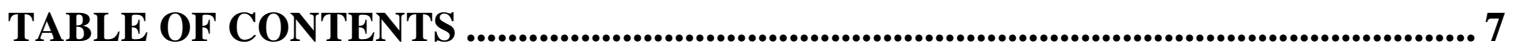

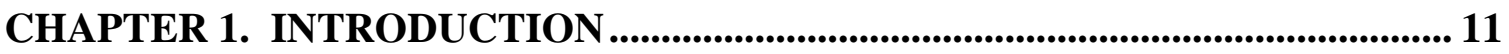

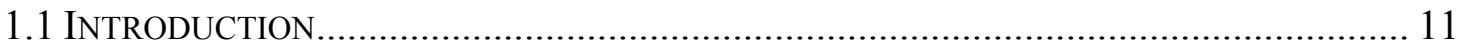

1.2 FIGURES

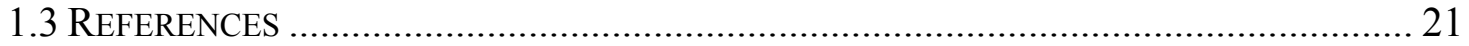

\section{CHAPTER 2. THE ABUNDANCE AND DIVERSITY OF MICROBIAL LIFE IN}

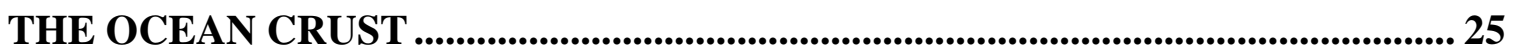

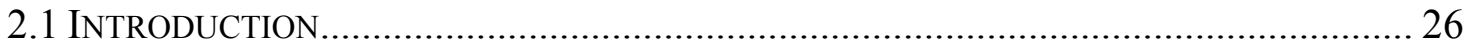

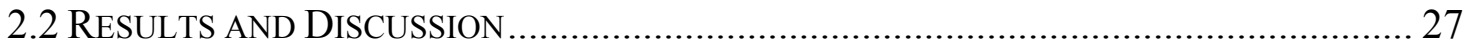

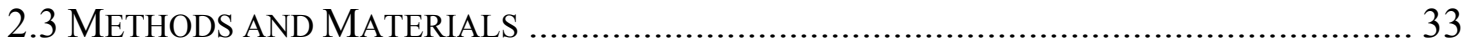

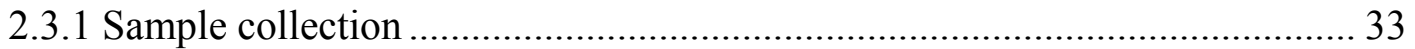

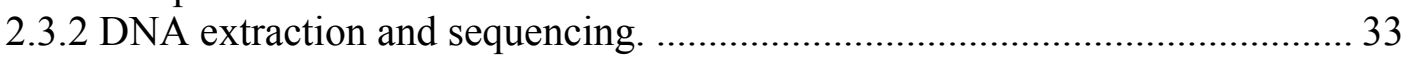

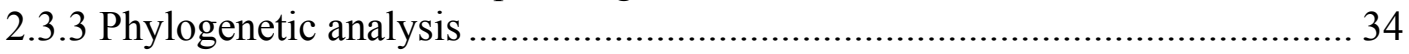

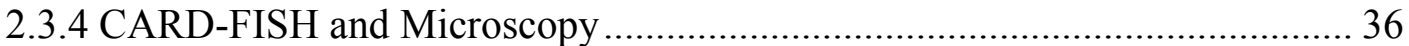

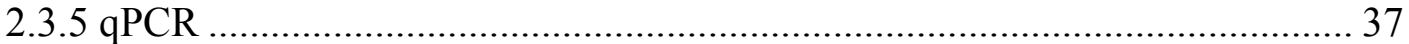

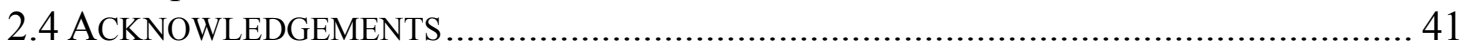

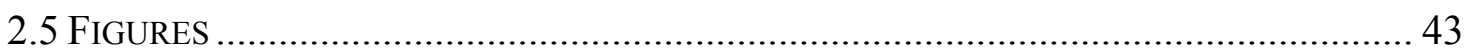

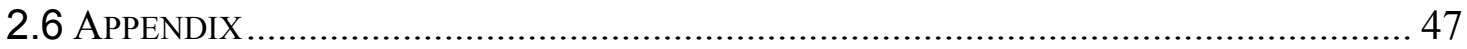

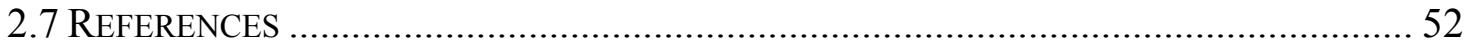

CHAPTER 3. THE DIVERSITY AND ABUNDANCE OF BACTERIA INHABITING SEAFLOOR LAVAS POSITIVELY CORRELATE WITH THE

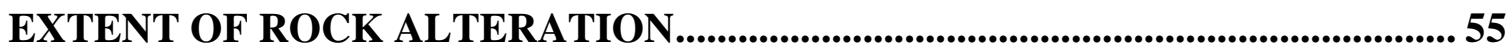

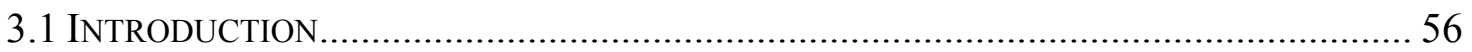

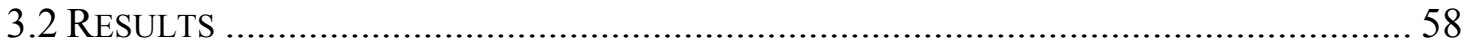

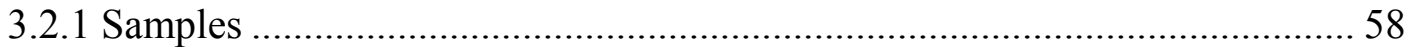

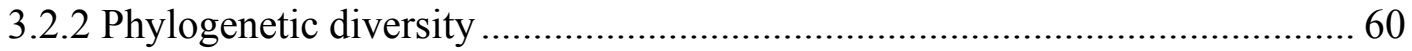

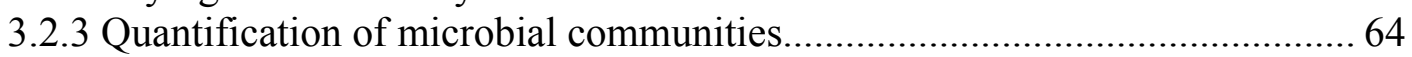

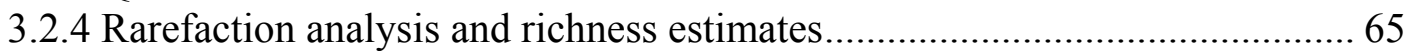

3.2.5 Bacterial community overlap and distribution estimates .................................. 66

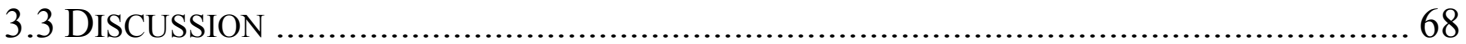

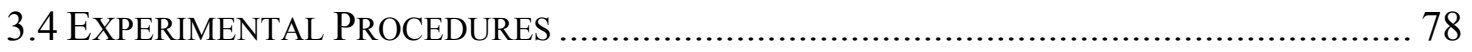

3.4.1 Study site and sample collection ............................................................... 78

3.4.2 Clone libraries and phylogenetic analyses ................................................. 78

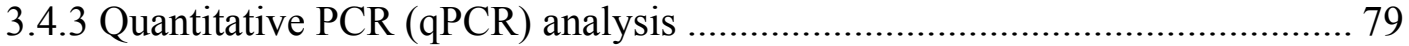

3.4.4 Rarefaction analysis and Diversity comparisons ........................................... 80 


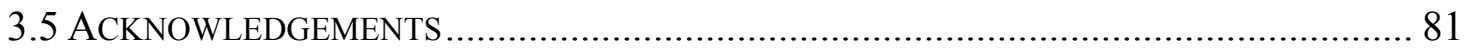

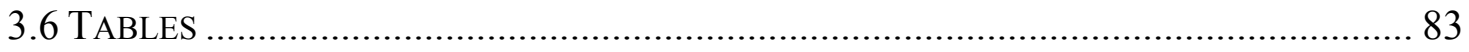

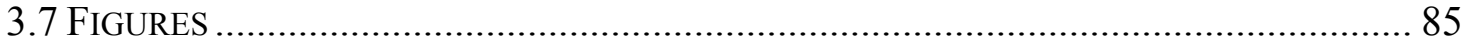

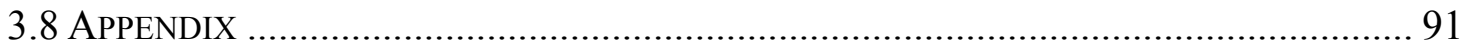

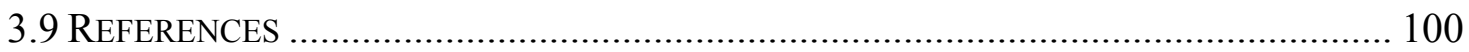

CHAPTER 4. THE SUBSURFACE OCEAN CRUST BIOSPHERE: EVIDENCE SUGGESTING LOW BIOMASS IN OLD (>10 MA) OCEAN CRUST ................... 105

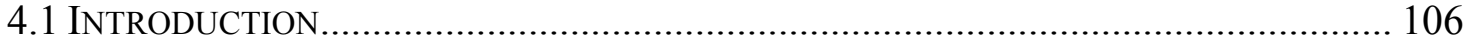

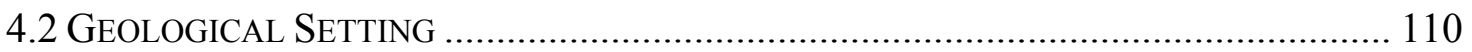

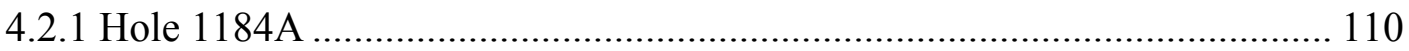

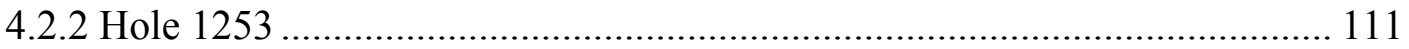

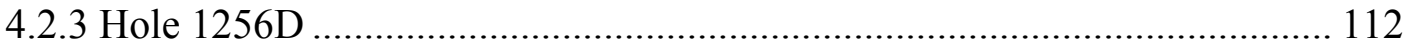

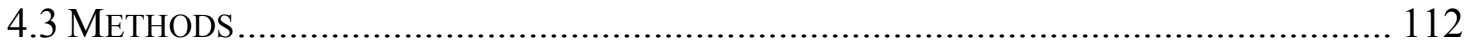

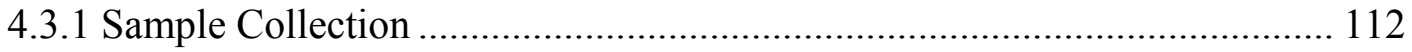

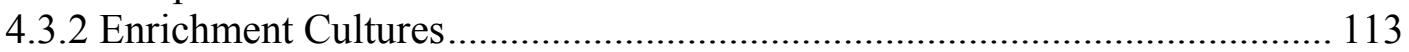

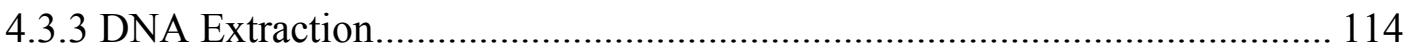

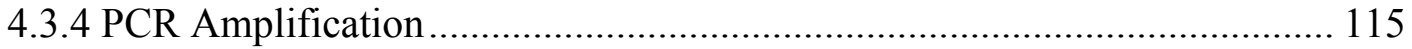

4.3.5 Extraction/Amplification Efficiency Test .................................................. 116

4.3.6 Clone Library Construction and Sequencing ............................................. 117

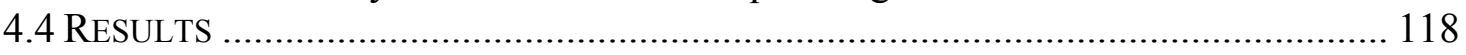

4.4.1 DNA extraction/amplification efficiency experiments ………………….... 118

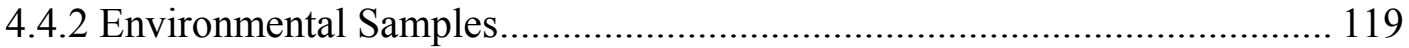

4.4.3 Phylogenetic Analyses ....................................................................... 119

4.4.4 Enrichment cultures.............................................................................. 121

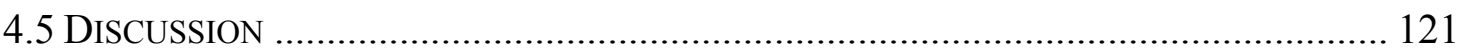

4.5.1 Protocol Development and Extraction Method Experiments........................ 121

4.5.2 Phylogenetic Analyses ........................................................................... 123

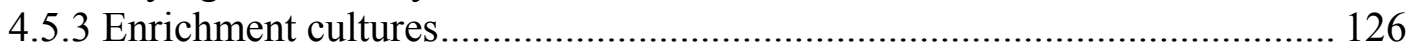

4.5.4 Other evidence suggesting minimal biomass ........................................... 127

4.5.5 Unique approaches to access the deep subsurface ....................................... 128

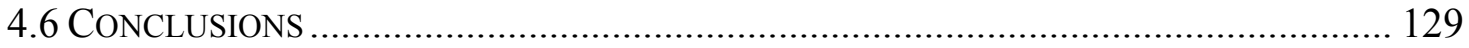

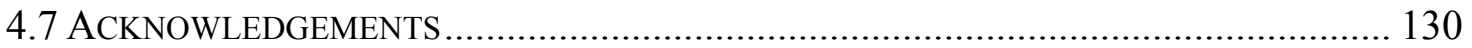

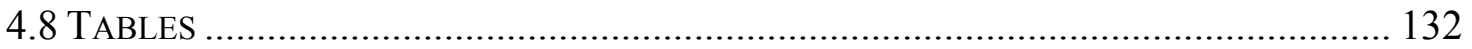

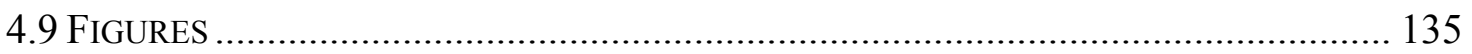

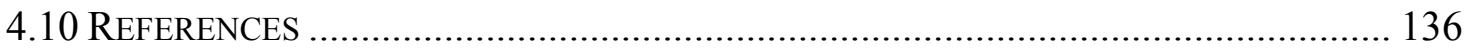

CHAPTER 5. CHARACTERIZATION OF LOW-TEMPERATURE

ALTERATION PRODUCTS ASSOCIATED WITH YOUNG BASALTIC

GLASSES FROM THE EAST PACIFIC RISE AT 9 ${ }^{\circ} \mathrm{N}$ : RESULTS OF

SYNCHROTRON-BASED $\mu$ XRF AND $\mu$ XAS STUDIES............................................ 141

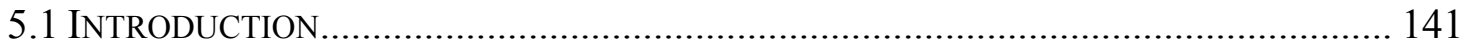

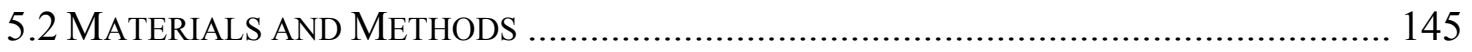

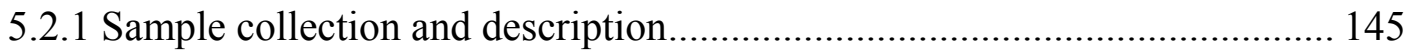


5.2.2 Synchrotron-based micro X-ray fluorescence ( $\mu$-SRXF) .......................... 146

5.2.3 Micro X-ray absorption spectroscopy ( $\mu$ XAS) .......................................... 147

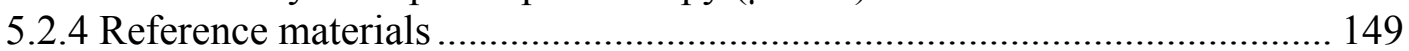

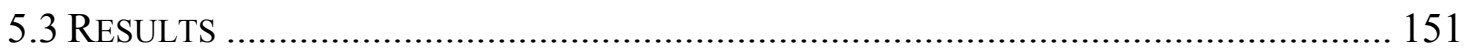

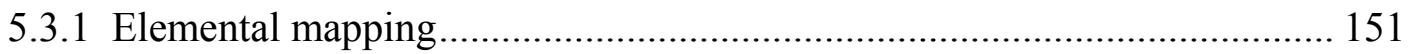

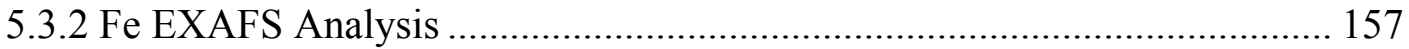

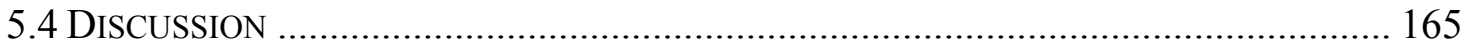

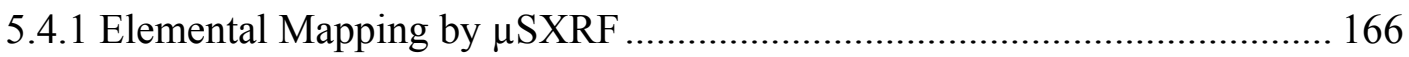

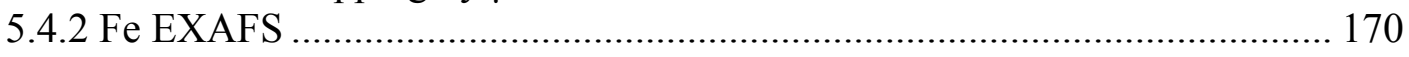

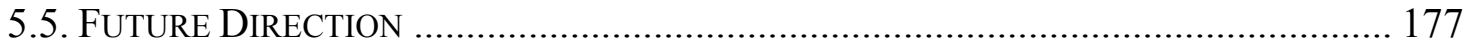

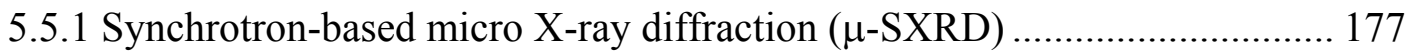

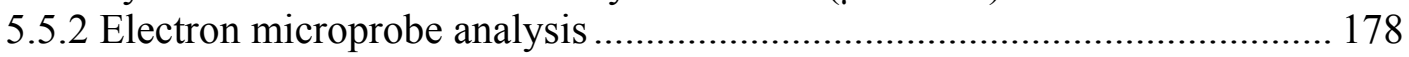

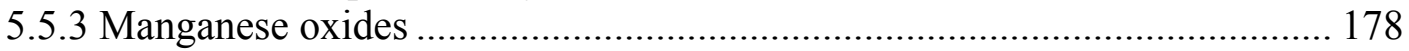

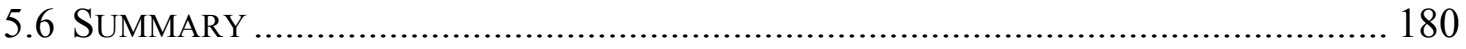

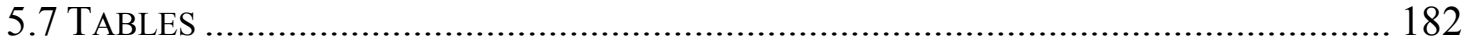

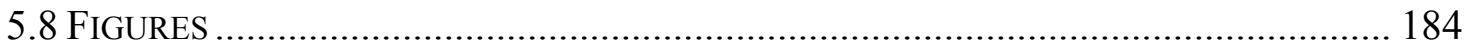

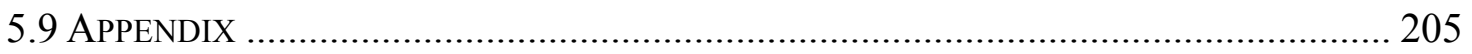

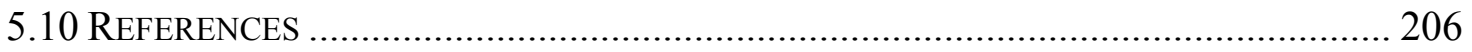

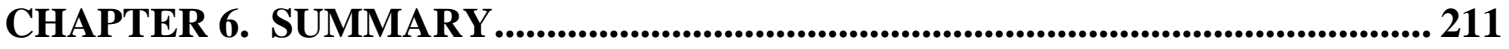

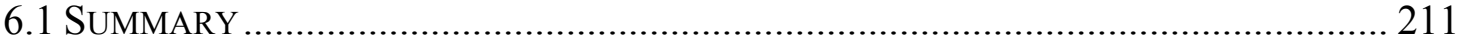

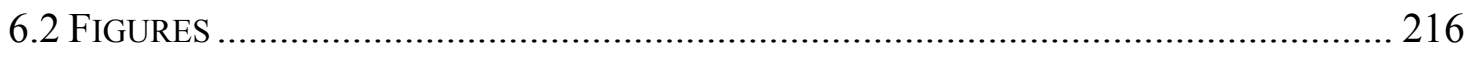

6.3 REFERENCES ......................................................................................... 217 


\section{Chapter 1. Introduction}

\subsection{Introduction}

The deep-sea biosphere is a topic that has garnered significant interest in the past years. Studies exploring the microbial communities that inhabit deep-sea environments have largely concentrated on deeply buried sediments (Biddle et al., 2006; D'Hondt et al., 2004; Kormas et al., 2003; Newberry et al., 2004; Schippers and Neretin, 2006; Teske, 2006) and mid- to high-temperature hydrothermal systems (Cowen et al., 1999; Nakagawa et al., 2004; Nercessian et al., 2003; Reysenbach et al., 2000; Schrenk et al., 2003; Sievert et al., 1999). Other studies also suggest that an ocean crust-hosted biosphere exists that could extend laterally beyond the MOR environment and extend deep into the basaltic crust (Bach and Edwards, 2003; Banerjee and Muehlenbachs, 2003; Cowen et al., 2003; Fisk et al., 1998; Furnes and Staudigel, 1999; Furnes et al., 2001;

Giovannoni et al., 1996; Huber et al., 2003; Huber et al., 2006; Lysnes et al., 2004a; Thorseth et al., 1992; Thorseth et al., 2003; Torsvik et al., 1998). Despite the large number of studies on the ocean crust biosphere, only a handful of these (Cowen et al., 2003; Huber et al., 2006; Lysnes et al., 2004a; Lysnes et al., 2004b) have provided direct evidence of the microbial commuities inhabiting seafloor and subseafloor crust. In fact, this biotope remains largely unexplored with respect to the types (phylogenetically and physiologically) of microorganisms, the activity and abundances of microorganisms, the lateral and vertical extent of the biosphere, and the effect that microbial activity has on the geochemistry of the ocean crust and overlying water column. This is suprising, 
considering the ocean crust covers $>60 \%$ of the Earth's surface and has the potential to host one of the largest endolithic communities on the planet.

The majority of evidence supporting the existence of an endolithic community in ocean crust has been limited to petrographic studies of basalt glass from drill cores of volcanic crust, with supporting evidence from isotopic and microbeam chemical analyses. In short, studies using transmitted light microscopy and scanning electron microscopy (SEM) have revealed either irregular granular or tubular, segmented channels observed throughout the glass alteration front and extending into fresh interiors (Banerjee and Muehlenbachs, 2003; Fisk et al., 1998; Furnes et al., 2001; Thorseth et al., 1995). These features have been observed in a wide variety of geographical settings and geological environments (depth, crustal age, etc). Geochemical analyses of these alteration features commonly reveal an enrichment of biologically relevant elements such as $\mathrm{C}, \mathrm{N}, \mathrm{P}, \mathrm{S}$, and $\mathrm{K}$ relative to fresh, unaltered glass. Additionally, stable carbon isotope analyses of disseminated carbonate within the altered bulk rock reveal $\delta^{13} \mathrm{C}$ values more depleted than either fresh basalt or expected values for inorganically precipitated marine carbonate. These anomalous alteration patterns and geochemical signatures have been interpreted to be a consequence of microbial activity within the ocean crust (Banerjee and Muehlenbachs, 2003; Fisk et al., 1998; Furnes and Staudigel, 1999; Furnes et al., 2001; Giovannoni et al., 1996; Thorseth et al., 1992; Thorseth et al., 2003; Torsvik et al., 1998). This hypothesis, however, is subject to debate as there is a lack of direct evidence (for example, laboratory based studies) linking any microbe with the capability of producing such alteration textures or signatures in basalt glass. Neither abiotically nor biologically 
mediated laboratory experiments carried out thus far have been successful at replicating these long (tens to hundreds of micrometers long), tubular alteration features (Staudigel et al., 1995; Thorseth et al., 1995) in glass.

Furthermore, few reports have indisputably determined the phylogenetic diversity of the seafloor and subseafloor ocean crust environments using molecular biological techniques. One of the few successful subsurface rock-hosted biosphere studies of the Ocean Drilling Program was ODP Leg 187 at the Southeast Indian Ridge (Lysnes et al., 2004b), in which the authors cultured bacteria and archaea as well as extracted and amplified bacterial DNA from 18-28 Ma ocean crust for phylogenetic analyses. Several additional studies have examined subsurface microbial communities by analyzing the microorganisms recovered from fluids emanating from young, ridge flank ocean crust (Cowen et al., 2003; Huber et al., 2003; Huber et al., 2006). Yet, very few studies have directly examined the communities existing in young, fresh seafloor lavas. One study (Lysnes et al., 2004a) recovered a somewhat diverse population of bacteria and archaea from a variety of young, seafloor basalts from the Arctic spreading ridges. A separate study (Templeton et al., 2005) was able to culture a variety of Mn-oxidizing bacteria from lavas at the Loihi Seamount. Still, very little is known about the abundances of microorganisms existing on the surfaces and within the fractures and pore spaces of the ocean crust, and even less is know about whether these communities are active in situ. Additionally, the mechanisms for providing the energy to fuel these ecosystems are poorly understood - what are the metabolic underpinnings of these rock-hosted communities? 
Ocean crust near mid-ocean ridges (MORs) and on ridge flanks is composed largely of glassy, basaltic lava flows that are highly enriched in reduced species of elements such as $\mathrm{Fe}, \mathrm{S}$, and $\mathrm{Mn}$. Upon exposure to oxygenated deep seawater, a variety of oxidation and hydration (hydrogen generating) reactions occur on the surfaces and within the fractures and void spaces of the basalt, particularly in the glassy portions (Alt, 1995). These oxic conditions prevail in recharge zones on the ridge flanks at the surface and subsurface out to nearly 10-20 Ma crust (as illustrated in Figure 1.1 by panel (b)). Chemolithoautotrophic microorganisms can potentially exploit the free energy changes associated with these low-temperature redox reactions in the ocean crust for their catabolic requirements. Indeed, a recent study by Bach and Edwards (2003) has demonstrated the potential for chemolithoautotrophic biomass production through the oxidation of $\mathrm{Fe}, \mathrm{S}$, and $\mathrm{H}_{2}$ in basaltic ocean crust. They estimate that these oxidation reactions, if entirely mediated by microorganisms, can support the production of nearly $10^{12} \mathrm{~g} \mathrm{C} \mathrm{yr}^{-1}$. We hypothesize that chemosynthetic communities could serve as the trophic base for ecosystems in this bare-rock environment. This chemosynthetic relationship has previously been demonstrated in a number of terrestrial subsurface environments (Edwards et al., 2000; Lovley and Chapelle, 1995; Pedersen et al., 1997), but this potential has only rarely been empirically examined in the deep-sea (Eberhard et al., 1995; Wirsen et al., 1993).

In addition to basalt oxidation reactions, other potential energy sources capable of sustaining microbial life exist in this bare-rock environment. The magmatic/hydrothermal input of hydrogen, methane, hydrogen sulfide, and carbon 
dioxide into the system could invoke microbial growth through a variety of metabolisms near the vent fields and off-axis seamounts as reduced vent fluids mix with oxygenated seawater (e.g., (a) and (c) of Figure 1.1). Dissolved organic carbon (DOC) in seawater or hydrothermal fluids (Lang et al., 2006) could also provide energy for slow-growing heterotrophic microorganisms at the seafloor. It is suggested, however, that most DOC in the deep-sea is recalcitrant to microbial oxidation (Barber, 1968), but this remains largely unconstrained. Further off-axis the input of sedimentary organic matter could sustain heterotrophic respiration in the ocean crust (as in panel (d) of Figure 1.1). Only phototrophy can be entirely ruled out as a potential source of metabolic energy.

One of the over-arching reseach objectives of this thesis was to test our hypothesis that chemosynthetic communities driven by basalt-related alteration reactions are fueling the ocean crust biotope. To do so first required the determination of the major types, abundances, and activities of microorganisms that exist in ocean crust. Chapter 2 describes how a number of molecular biological techniques were used to establish these unknowns in young $(<20 \mathrm{kyr})$, seafloor ocean crust from the East Pacific Rise (EPR) at $9^{\circ} \mathrm{N}$. Near ull-length $16 \mathrm{~S}$ rRNA gene sequencing was first used to identify the major phylogenetic groups of Bacteria inhabiting several variably altered basaltic lavas, as well as the surrounding deep seawater. These efforts were combined with quantitative polymerase chain-reaction (qPCR) and catalyzed reporter deposition-fluorescent in situ hybridization (CARD-FISH) to estimate the total and active prokaryotic communities in this environment. These analyses allowed us to statistically compare our findings to relevant, well-studied oceanic environments, such as hydrothermal vents and seawater 
(both surface and bottom), for which near full-length 16S rDNA sequences are publicly available.

One of the goals of chapter 3 was to more closely examine the species diversity and abundances of bacterial communities recovered from the seafloor lavas analyzed in Chapter 2. These efforts took advantage of there being a variety of different alteration states and mineralogical oxidation products exhibited in these lavas to determine the relationship between microbial growth and basalt alteration processes. Here, the phylotype richness, membership, and community structure for each basalt sample were compared using statistical tools to determine patterns of association between taxa, microbial abundances, and extent of basalt glass alteration. Additionally, the potential physiologies of microorganisms existing on glass surfaces and within the fractures and pores spaces were identified through phylogenetic inference. This provides preliminary evidence for the existence of chemolithoautotrophic microorganisms and allows for the identification of a number of microbial metabolisms that could impact the geochemical cycling of elements in the deep sea.

It has been hypothesized that microbial communities can exist within ocean crust as long as hydrothermal circulation is supported (Staudigel et al., 2004). Heat flow measurements suggest that fluid circulation occurs in crust less than or equal to $\sim 65 \pm \mathrm{Ma}$ (Stein et al., 1995), and porosity and permeability measurements infer possible circulation to depths up to $200-500 \mathrm{~m}$, e.g. (Alt, 1995). Unfortunately, current drilling technologies preclude the sampling of subsurface lavas in very young ocean crust, for example at the East Pacific Rise. However, older subsurface crust formed at the EPR 
millions of years ago (and now capped by a thick sediment cover, e.g., as shown in Figure 1.1, panel d) has been collected during several Ocean Drilling Program (ODP) research cruises. For Chapter 4, a number of basaltic rock and glass drill-core samples were collected from three Pacific Ocean drilling sites for both culture dependent and culture-independent 16S rRNA phylogenetic analyses. These samples represent a variety of different environments (age, likely fluid flux, extent of alteration, depth, etc.) within the ocean crust and allow us to further elucidate the extent of the subsurface endolithic microbial biotope.

If microorganisms inhabiting ocean crust are supported by basalt alteration reactions, then it is possible that these communities impact the geochemical evolution of the crust. The role that these microorganisms play in the early stages of basalt weathering, however, is poorly constrained, principally because the initial processes and mechanisms controlling low-temperature alteration in the environment are not well studied. The majority of studies examining basalt glass dissolution and alteration have either been laboratory based experiments that typically do not incorporate biological influences (Crovisier et al., 2003 and references therein; Gislason and Arnorsson, 1993; Gislason and Oelkers, 2000; Seyfried Jr. and Bischoff, 1979; Seyfried Jr. and Bischoff, 1981) or studies of naturally weathered basaltic rocks and glasses that utilize primarily electron-based methods and analytical techniques (Alt and Mata, 2000; Alt and Teagle, 2003; Banerjee and Muehlenbachs, 2003; Giorgetti et al., 2001; Storrie-Lombardi and Fisk, 2004). While these studies have significantly progressed our understanding of glass alteration processes, the early stages of in situ basalt alteration (most relevant for 
biological processes) are not well documented, especially regarding the production of secondary mineralogical products (which are often poorly crystalline and notoriously difficult to characterize using electron microscopy or diffraction) and speciation of elements like $\mathrm{Fe}, \mathrm{Mn}$, and $\mathrm{S}$ across the alteration front. In chapter 5, synchrotron based X-ray fluorescence (SXRF) and X-ray absorption spectroscopy (XAS) was used for spatially-resolved, micrometer scale analyses of low-temperature volcanic glass alteration. Our goal was to identify and characterize the minerals produced in the early stages of basalt glass weathering in geologically young $(<20 \mathrm{kyrs})$ volcanic glasses collected from the seafloor at the EPR.

The goal of this thesis was to establish the microbial diversity, abundance, and activity of microbial communities inhabiting the ocean crust while providing direct evidence in support of our hypothesis that this ecosystem is underpinned by chemosynthetic communities fueled by basalt alteration reactions. Because of the interdisciplinary nature of the subject matter, a wide variety of molecular and microbiological methods in combination with different mineralogical, geochemical, and microscopic techniques were utilized to obtain our objectives. The results of these studies greatly contribute our understanding of the geomicrobiology of ocean crust and highlight the significance of this biosphere in a global context. 


\subsection{Figures}

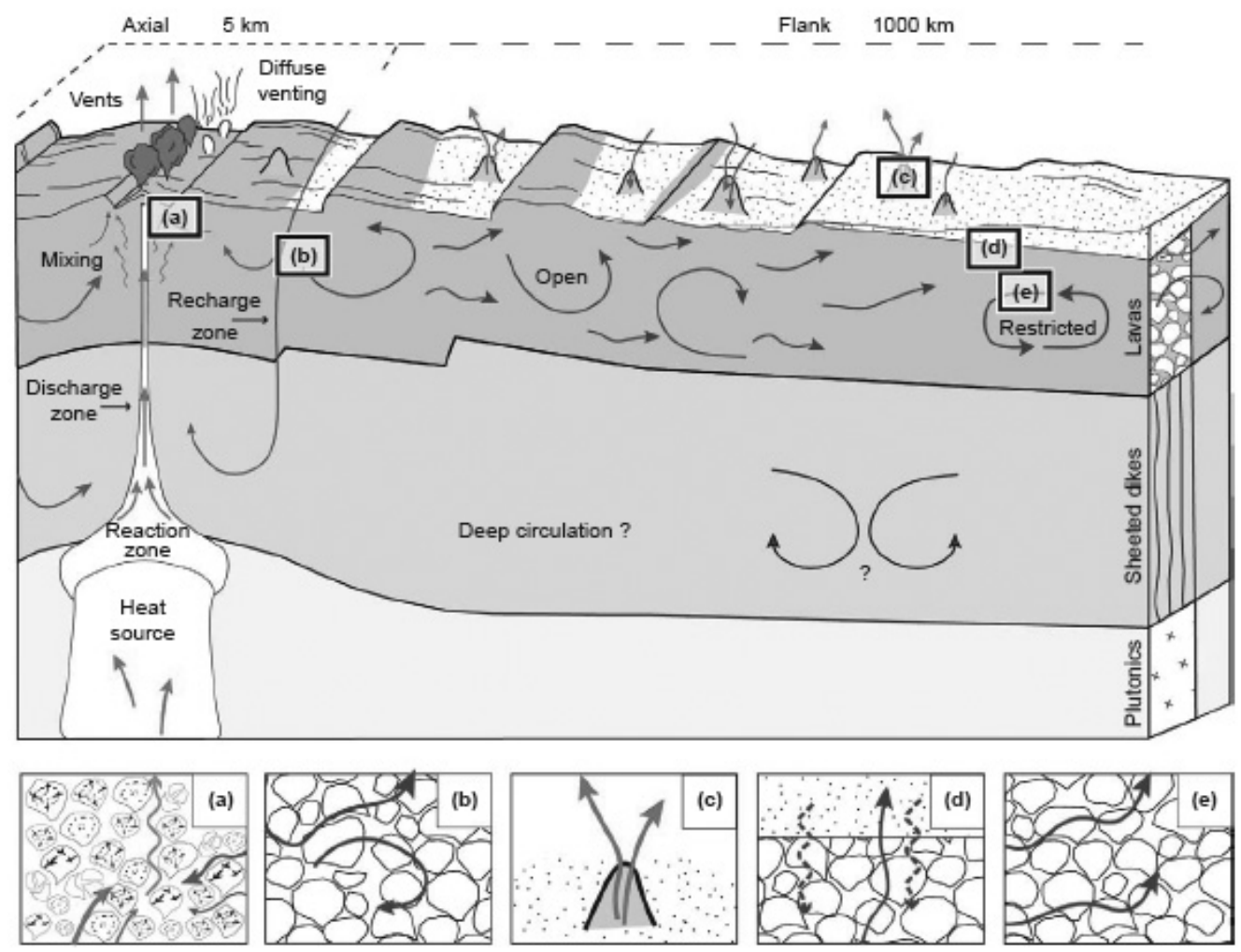

Figure 1.1 Schematic cross-section of the ocean crust showing fluid flow regimes (from Edwards et al., (2005)). (a) Axis of the mid-ocean system where hot, reducing fluids mix with cold oxygenated seawater. (b) Recharge zones and young ridge flanks where cold, oxygenated seawater interacts with basaltic rock. (c) Off-axis discharge at seamounts. (d) Ocean crust capped by thick sediment layer. (e) Old ocean crust where hydrothermal circulation is restricted. 


\subsection{References}

Alt, J.C., 1995. Subseafloor processes in mid-ocean ridge hydrothermal systems. In: S.E. Humphris, R.A. Zierneberg, L.S. Mullineaux and R.E. Thomson (Editors), Seafloor hydrothermal systems: physical, chemical, biological, and geological interactions. AGU Monograph. American Geophysical Union, Washington, DC, pp. 85-114.

Alt, J.C. and Mata, P., 2000. On the role of microbes in the alteration of submarine basaltic glass: a TEM study. Earth and Planetary Science Letters, 181(301-313).

Alt, J.C. and Teagle, D.A.H., 2003. Hydrothermal alteration of upper oceanic crust formed at a fast-spreading ridge: mineral, chemical, and isotopic evidence from ODP Site 801. Chem. Geol., 201: 191-211.

Bach, W. and Edwards, K.J., 2003. Iron and sulfide oxidation within the basaltic ocean crust: Implications for chemolithoautotrophic microbial biomass production. Geochim. Cosmochim. Acta, 67(20): 3871-3887.

Banerjee, N.R. and Muehlenbachs, K., 2003. Tuff life: Bioalteration in volcaniclastic rocks from the Ontong Java Plateau. Geochemistry Geophysics Geosystems, 4: Art. No. 1037(art. no. 1037).

Barber, R.T., 1968. Dissolved organic carbon from deep water resists microbial oxidation. Nature, 220(274-275).

Biddle, J.F. et al., 2006. Heterotrophic archaea dominate sedimentary subsurface ecosystems off Peru. Proc. Natl. Acad. Sci., 103(10): 3846-3851.

Cowen, J.P. et al., 2003. Fluids from aging ocean crust that support microbial life. Science, 299(5603): 120-123.

Cowen, J.P. et al., 1999. Microbial biomass in the hydrothermal plumes associated with the 1998 Axial Volcano eruption. Geophys. Res. Lett., 26(24): 3637-3640.

Crovisier, J.-L., Advocat, T. and Dussossoy, J.-L., 2003. Nature and role of natural alteration gels formed on the surface of ancient volcanic glasses (Natural analogs of waste containment glasses). Journal of Nuclear Materials, 321: 91-109.

D'Hondt, S. et al., 2004. Distributions of microbial activities in deep subseafloor sediments. Science, 306: 2216-2221.

Eberhard, C., Wirsen, C.O. and Jannasch, H.W., 1995. Oxidation of polymetal sulfides by chemolithoautotrophic bacteria from deep-sea hydrothermal vents. Geomicrobiology Journal, 13(3): 145-164.

Edwards, K.J., Bach, W. and McCollom, T.M., 2005. Geomicrobiology in oceanography: microbe-mineral interactions at and below the seafloor. TRENDS in Microbiology, 13(9).

Edwards, K.J., Bond, P.L., Gihring, T.M. and Banfield, J.F., 2000. An archaeal ironoxidizing extreme acidophile important in acid mine drainage. Science, 287(5459): 1796-1799.

Fisk, M.R., Giovannoni, S.J. and Thorseth, I.H., 1998. Alteration of Oceanic Volcanic Glass? Textural Evidence of Microbial Activity. Science, 281(978-980).

Furnes, H. and Staudigel, H., 1999. Biological mediation in ocean crust alteration: how deep is the deep biosphere. Earth and Planetary Science Letters, 166: 97-103. 
Furnes, H. et al., 2001. Bioalteration of basaltic glass in the oceanic crust. Geochemistry, Geophysics, Geosystems, 2: Art. No. 2000GC000150.

Giorgetti, G., Marescotti, P., Cabella, R. and Lucchetti, G., 2001. Clay mineral mixtures as alteration products in pillow basalts from the eastern flank of Juan de Fuca Ridge: a TEM-AEM study. Clay Minerals, 36(75-91).

Giovannoni, S.J., Fisk, M.R., Mullins, T.D. and Furnes, H., 1996. Genetic evidence for endolithic microbial life colonizing basaltic glass/sea water interfaces.

Proceedings of the Ocean Drilling Program, Scientific Results, 148: 207-213.

Gislason, S.R. and Arnorsson, S., 1993. Dissolution of primary basaltic minerals in natural waters: saturation state and kinetics. Chem. Geol., 105: 117-135.

Gislason, S.R. and Oelkers, E.H., 2000. Chemical Weathering rate of basaltic glass as a function of temperature, $\mathrm{pH}$, organic acids and solution composition. Journal of Conference Abstracts, 5(2): 443.

Huber, J., Butterfield, D. and Baross, J., 2003. Bacterial diversity in a subseafloor habitat following a deep-sea volcanic eruption. FEMS Microbiol. Ecol., 43(3): 393-409.

Huber, J.A., Johnson, H.P., Butterfield, D.A. and Baross, J.A., 2006. Microbial life in ridge flank crustal fluids. Appl. Environ. Microbiol., 8(1): 88-99.

Kormas, K.A., Smith, D.C., Edgcomb, V. and Teske, A., 2003. Molecular analysis of deep subsurface microbial communities in Nankai Trough sediments (ODP Leg 190, Site 1176). FEMS Microbiology Ecology, 45: 115-125.

Lang, S.Q., Butterfield, D.A., Lilley, M.D., Johnson, H.P. and Hedges, J.I., 2006. Dissolved organic carbon in ridge-axis and ridge-flank hydrothermal systems. Geochim. Cosmochim. Acta, 70: 3830-3842.

Lovley, D.R. and Chapelle, F.H., 1995. Deep subsurface microbial processes. Reviews of Geophysics, 33(3): 365-381.

Lysnes, K. et al., 2004a. Microbial community diversity in seafloor basalt from the Arctic spreading ridges. FEMS Microbiology Ecology, 50: 213-230.

Lysnes, K., Torsvik, T., Thorseth, I.H. and Pedersen, R.B., 2004b. Microbial populations in ocean floor basalt: Results from ODP Leg 187. In: R.B. Pedersen, D.M. Christie and D.J. Miller (Editors), Proceedings of the Ocean Drilling Program, Scientific Results.

Nakagawa, T., Nakagawa, S., Inagaki, F., Takai, K. and Horikoshi, K., 2004. Phylogenetic diversity of sulfate-reducing prokaryotes in active deep-sea hydrothermal vent chimney structures. FEMS Microbiology Letters, 232: 145152.

Nercessian, O., Reysenbach, A., Prieur, D. and Jeanthon, C., 2003. Archaeal diversity associated with in situ samplers deployed on hydrothermal vents on the East Pacific Rise ( $\left.13^{\circ} \mathrm{N}\right)$. Environmental Microbiology, 5(6): 492-502.

Newberry, C.J. et al., 2004. Diversity of prokaryotes and methanogenesis in deep subsurface sediments from the Nankai Trough, Ocean drilling Program Leg 190. Environmental Microbiology, 6(3): 274-287.

Pedersen, K. et al., 1997. Evidence of ancient life at $207 \mathrm{~m}$ depth in a granitic aquifer. Geology, 25(9): 827-830. 
Reysenbach, A.-L., Longnecker, K. and Kirshtein, J., 2000. Novel bacterial and archaeal lineages from an in situ growth chamber deployed at a Mid-Atlantic ridge hydrothermal vent. Appl. Environ. Microbiol., 66(9): 3798-3806.

Schippers, A. and Neretin, L.N., 2006. Quantification of microbial communities in nearsurface and deeply buried marine sediments on the Peru continental margin using real-time PCR.

Schrenk, M.O., Kelley, D.S., Delaney, J.R. and Baross, J.A., 2003. Incidence and diversity of microorganisms within the walls of an active deep-sea sulfide chimney. Appl. Environ. Microbiol., 69(6): 3580-3592.

Seyfried Jr., W.E. and Bischoff, J.L., 1979. Low temperature basalt alteration by seawater: an experimental study at $70^{\circ} \mathrm{C}$ and $150^{\circ} \mathrm{C}$. Geochimica et Cosmochimica Acta, 43: 1937-1947.

Seyfried Jr., W.E. and Bischoff, J.L., 1981. Experimental seawater-basalt interaction at $300^{\circ} \mathrm{C}, 500$ bars, chemical exchange, secondary mineral formation and implication for the transport of heavy metals. Geochimica et Cosmochimica Acta, 45(2): 135-147.

Sievert, S.M., Brinkhoff, T., Muyzer, G., Ziebis, W. and Kuever, J., 1999. Spatial heterogeneity of bacterial populations along an environmental gradient at a shallow submarine hydrothermal vent near Milos Island (Greece). Applied and Environmental Microbiology, 65(9): 3834-3842.

Staudigel, H., Chastain, R.A., Yayanos, A. and Bourcier, W., 1995. Biologically mediated dissolution of glass. Chemical Geology, 126: 147-154.

Staudigel, H. et al., 2004. The ocean crust as a bioreactor. In: W.S.D. Wilcock, E.F. DeLong, D.S. Kelley, J.A. Baross and S.C. Cary (Editors), The Subseafloor Biosphere at Mid-Ocean Ridges. American Geophysical Union, pp. 325-341.

Stein, C.A., Stein, S. and Pelayo, A., 1995. Heat flow and hydrothermal circulation. In: S.E. Humphris, R.A. Zierenberg, L.S. Mullineaux and R.E. Thomson (Editors), Seafloor Hydrothermal Systems: Physical, Chemical, Biological, and Geological Interactions. American Geophysical Union, pp. 425-445.

Storrie-Lombardi, M.C. and Fisk, M.R., 2004. Elemental abundance distributions in suboceanic basalt glass: Evidence of biogenic alteration. Geochemistry, Geophysics, Geosystems, 5(10).

Templeton, A.S., Staudigel, H. and Tebo, B.M., 2005. Diverse Mn(II)-oxidizing bacteria isolated from submarine basalts at Loihi Seamount. Geomicrobiology Journal, 22: 127-139.

Teske, A.P., 2006. Microbial communities of deep marine subsurface sediments: Molecular and cultivation surveys. Geomicrobiology Journal, 23: 357-368.

Thorseth, I.H., Furnes, H. and Heldal, M., 1992. The importance of microbiological activity in the alteration of natural basaltic glass. Geochim. Cosmochim. Acta, 56: 845-850.

Thorseth, I.H., Furnes, H. and Tumyr, O., 1995. Textural and chemical effects of bacterial activity on basaltic glass: an experimental approach. Chemical Geology, 119: 139-160. 
Thorseth, I.H., Pedersen, R.B. and Christie, D.M., 2003. Microbial alteration of 0-30-Ma seafloor and sub-seafloor basaltic glasses from the Australian Antarctic Discordance. Earth and Planetary Science Letters, 215: 237-247.

Torsvik, T., Hurnes, H., Muehlenbachs, K., Thorseth, I.H. and Tumyr, O., 1998.

Evidence for microbial activity at the glass-alteration interface in oceanic basalts. Earth and Planetary Science Letters, 162: 165-176.

Wirsen, C.O., Jannasch, H.W. and Molyneaux, S.J., 1993. Chemosynthetic microbial activity at Mid-Atlantic Ridge hydrothermal vent sites. Journal of Geophysical Research-Solid Earth, 98(B6): 9693-9703. 


\title{
Chapter 2. The abundance and diversity of microbial life in the ocean crust
}

\author{
Cara M. Santelli ${ }^{1,2}$, Wolfgang Bach ${ }^{3}$, Craig L. Moyer ${ }^{4}$, Katrina J. Edwards ${ }^{2,5^{*}}$ \\ ${ }^{I}$ MIT/WHOI Joint Program in Oceanography and Ocean Engineering \\ ${ }^{2}$ Geomicrobiology Group, Woods Hole Oceanographic Institution, MS \#52, Woods Hole, \\ Massachusetts, 02543, USA \\ ${ }^{3}$ Fachbereich Geowissenschaften, Universität Bremen, Postfach 3304 40, D-28334 \\ Bremen, Germany \\ ${ }^{4}$ Department of Biology, Western Washington University, MS\#9160 \\ Bellingham, WA 98225, USA \\ ${ }^{5 *}$ Geomicrobiology Group, Department of Biological Sciences, Marine Environmental \\ Biology, University of Southern California, 3616 Trousdale Blvd, Los Angeles, CA, \\ 90089-0371 USA
}

\begin{abstract}
Oceanic lithosphere exposed at the seafloor undergoes seawater-rock reactions that may supply metabolic energy to support microbial growth. While it is recognized that deep-sea sediments (D'Hondt et al., 2004; Ravenschlag et al., 1999) and hightemperature hydrothermal systems (Reysenbach et al., 2000; Schrenk et al., 2003) support a range of microbial ecosystems that occupy specific environmental niches, the bare rock ocean crust microbial biotope is nearly unexplored. Here we show that exposed basaltic lavas at the East Pacific Rise (EPR) harbor microbial communities that are characterized by exceptionally diverse populations dominated by Bacteria. The overall species diversity is comparable to, but phylogenetically distinct from, soil communities, which are arguably considered to be the most diverse microbial ecosystems on Earth (Torsvik et al., 1990). Additionally, rock-hosted microorganisms are distinct
\end{abstract}


and more abundant than those present in the surrounding environments; quantitative polymerase chain reaction (qPCR), in situ hybridizations, and microscopy demonstrate cell abundances in the basalt ecosystem that are 3-4 orders of magnitude greater than in overlying deep-sea water. We hypothesize that these communities are underpinned by alteration reactions (oxidation and hydration) in and on basaltic ocean crust. That is, chemolithoautotropic microorganisms constitute the trophic base and further stimulate biomass production in this habitat. The degree to which these microbes participate in and promote oxidative alteration reactions is not known, but they may significantly affect the rates and pathways of chemical exchange between the basaltic crust and seawater.

\subsection{Introduction}

To elucidate the role of microorganisms in alteration reactions associated with ocean crust seafloor weathering, we assessed the phylogenetic diversity, species richness, and abundance of endo- and epi-lithic microbial communities inhabiting young, unsedimented ocean crust at the seafloor. Basaltic seafloor lavas representing various ages (up to 20 kyrs), alteration states, and local environments were collected from the EPR (Fig. 2.1a-c and Appendix 2 Fig. A2.1) for microscopic (Fig. 2.1d,e) and molecular biological analyses. Full-length $16 \mathrm{~S}$ rRNA clone libraries were generated and sequenced to identify the bacterial community inhabiting ocean crust, as well as the surrounding deep seawater. These efforts were combined with catalyzed reporter depositionfluorescent in situ hybridization (CARD-FISH) and qPCR to estimate the total and active communities in this environment. The methods used here allow us to statistically 
compare our findings to relevant, well-studied oceanic environments for which near fulllength 16S rDNA sequences are publicly available.

\subsection{Results and Discussion}

Statistical approaches to evaluate species richness, the number of operational taxonomic units (OTUs), of the seafloor basalts as a collective ecosystem reveal a diverse bacterial community. In this study, we define an OTU at $97 \%$ sequence similarity, which is an accepted, although controversial, taxonomic distinction for species level considerations (Schloss and Handelsman, 2006). Rarefaction (Fig. 2.2) compares the bacterial richness of the ocean crust habitat relative to deep seawater from the EPR, the upper water column of the Sargasso Sea (Venter et al., 2004), moderate temperature hydrothermal vent fluids from the Mid-Atlantic Ridge (MAR) captured in an in situ growth chamber (Reysenbach et al., 2000), and a terrestrial farm soil environment (Tringe et al., 2005). Although neither environment is sampled to saturation, our analyses reveal that EPR basalts harbor bacterial diversity comparable to that of soil. This result may be unexpected given that soil communities are considered to support some of the most diverse microbial communities on Earth (e.g, Moyer et al., 1998; Torsvik et al., 1990). Furthermore, it is apparent that the seafloor lavas harbor substantially more bacterial diversity than the other marine habitats.

The relative diversity of these microbial communities revealed by rarefaction analysis is corroborated by nonparametric based estimates of species richness and diversity (Appendix 2 Table A2.1). Because nonparametric estimators produce widely 
varying results and can arguably underestimate true diversity of microbial ecosystems (Hong et al., 2006; Hughes et al., 2001), we do not interpret these results as accurate predictions of true species richness. Rather, we use these indicators to support our rarefaction observations of the relative differences between the communities. This secondary comparison is informative because rarefaction curves can intersect and cross with increased sampling effort, particularly in high diversity communities where curves do not reach an asymptotic high (Hughes and Hellmann, 2005). Results show that the relative estimated diversity is consistent amongst the different environments, regardless of the estimator or index used, further supporting our observations that deep-ocean barerock basalt habitats harbor enormous genetic diversity and thus may be one of the most significant reservoirs of diversity in the oceans. One important caveat of all comparative measurements is that, other than the samples from the EPR, the specific methods used to generate sequences (i.e., DNA extraction protocol, PCR amplification, clone library construction) from the other studies differ slightly, which could influence the observed and predicted phylogenetic diversity. Our conclusions concerning the relative diversity of basalts compared with other habitats, however, seem unlikely to be altered by these differences.

Examination of the taxonomic groups obtained from various environments (Fig. 2.3) further reveals the considerable phylogenetic diversity of Bacteria in seafloor basalts, which is proportional to, yet distinct from, farm soil. The 17 taxonomic groups recovered from basalts were dominated by sequences belonging to the Proteobacteria division (including all subdivisions: $\alpha, \beta, \gamma, \delta$, and $\varepsilon$ ), which account for $68 \%$ of all 
sequences. A number of sequences were recovered from non-Proteobacteria groups such as the Plantomycetes (8\%), Actinobacteria (7\%), Bacteroidetes (4\%), Acidobacteria (3\%), and Verrucomicrobia (2\%). The other phyla recovered represent $<1 \%$ each of the total clone library. Approximately $6 \%$ of the sequences could not be assigned a taxonomic group and represent possible novel microorganisms.

In contrast to the ocean crust habitat, the phylogenetic distribution of Bacteria in the other deep-sea environments is rather low. The EPR deep seawater clone library reveals only three distinct taxonomic groups all belonging to the Proteobacteria ( $\gamma$ Proteobacteria, which account for $89 \%$ of the total library, $\alpha$-Proteobacteria, and $\beta$ Proteobacteria). Other deep seawater studies (Huber et al., 2003; Huber et al., 2006) have recovered additional taxa belonging to $\delta$-Proteobacteria, Bacteroidetes, and Planctomycetes. Subsequent rarefaction analysis including these sequences (Appendix 2 'deep seawater analysis' and Fig. A2.2), however, show that our interpretation is unaffected. The clone library of an in situ growth chamber placed above a hydrothermal vent at the MAR (Reysenbach et al., 2000), Fig. 2.3d, depicts a significantly different community representing fewer taxonomic groups. Particularly, numerous clones belonging to Aquificales and $\varepsilon$-Proteobacteria, organisms typically found in hydrothermal environments, were recovered from the growth chamber. These clones are virtually absent in the other two deep-sea libraries (basalts and seawater).

We also compared differences in total cell numbers observed in deep seawater with the biomass associated with basalt surfaces. qPCR measurements of EPR basalts determined total prokaryote cell densities ranging from $\sim 3 \times 10^{6}$ to $\sim 1 \times 10^{9}$ cells $\mathrm{g}^{-1}$ 
$\left(10^{7}\right.$ to $3 \times 10^{9}$ gene copies $\left.\mathrm{g}^{-1}\right)$ rock for samples with increasing basalt alteration (Appendix 2 Table A2.2). Additionally, the results show that Bacteria dominate (8896\%) the prokaryotic community for the range of altered basalt samples. CARD-FISH analyses confirm that bacterial cells are significantly more dense on basalt surfaces, and only a small proportion of the metabolically active cells can be accounted for by Archaea (Fig. 2.4a-c). By comparison, cell densities in the upper ocean $(<150 \mathrm{~m})$ are estimated at $2-3 \times 10^{5}$ cells $\mathrm{mL}^{-1}$ dominated $(90 \%)$ by Bacteria (Karner et al., 2001). In the deep ocean $(>1000 \mathrm{~m})$, Archaea account for approximately half of the total prokaryotic population of $8 \times 10^{3}-9 \times 10^{4}$ cells $/ \mathrm{mL} \mathrm{mL}^{-1}$ seawater (Cowen et al., 2003; Karner et al., 2001). These low cell numbers in the deep ocean waters contrast starkly with the high biomass associated with volcanic glass detected in this study, and this raises questions about what energy source(s) fuel this diverse bacterial community inhabiting basalt.

We hypothesize that alteration reactions occurring on basalt surfaces contribute to biomass production within the basalt-hosted biotope, and examine the possibility from a theoretical perspective. Lava surfaces are composed predominantly of volcanic glass, which is a highly reactive rock component enriched in reduced species of elements such as $\mathrm{Fe}, \mathrm{S}$, and $\mathrm{Mn}$. Oxygen and nitrate in deep-sea water oxidizes these reduced constituents of the rock, and chemolithoautotrophic microorganisms could potentially exploit the free energy changes associated with these redox reactions for their metabolic requirements.

Support for this hypothesis is provided by previous laboratory studies which demonstrate that chemolithoautotrophic Fe-oxidizing bacteria isolated from the seafloor 
are able to use solid rock and minerals, including basalt glass, for metabolism and growth (Edwards et al., 2003). Furthermore, biomass production calculations (Bach and Edwards, 2003) suggest that the observed oxidation of Fe and S in the upper ocean crust, if entirely mediated by microorganisms, can support the fixation of up to $\sim 5 \times 10^{11} \mathrm{~g} \mathrm{C}$ $\mathrm{yr}^{-1}$. Assuming $\sim 4 \times 10^{15} \mathrm{~g}$ of fresh basalt $\mathrm{yr}^{-1}$ are subject to oxidation reactions (Bach and Edwards, 2003) and a single cell contains 200 fg C (Whitman et al., 1998), we estimate that effectively $6 \times 10^{7}$ cells $\mathrm{g}^{-1}$ rock can be supported through alteration reactions (supposing the microbial growth efficiency is 0.1 and the biomass turns over every year). These parameters can be adjusted for more conservative estimates preferring a more oligotrophic, slower growing environment in which more energy goes into cell maintenance rather than cell growth, as in deep sea sediments (Biddle et al., 2006). For example if carbon content is $20 \mathrm{fg} \mathrm{C}$ cell $^{-1}$, growth efficiency is 0.01 , and biomass turnover is 100 years, nearly $6 \times 10^{9}$ cells $\mathrm{g}^{-1}$ basalt could be produced from basalt oxidation reactions. Cell densities in volcanic glasses from the EPR established through qPCR measurements, $\sim 10^{6}$ to $10^{9}$ cells $\mathrm{g}^{-1}$ basalt, are in close agreement with predicted cell densities of $\sim 10^{7}$ to $10^{9}$ cells $\mathrm{g}^{-1}$ basalt based on $\mathrm{Fe}$ and $\mathrm{S}$ oxidation reactions.

These preliminary calculations strongly suggest that alteration reactions in the upper ocean crust indeed fuel microbial ecosystems at the seafloor and contribute to biomass production and diversity in these systems. Otherwise, the ocean crust biotope would be expected to more closely match that of the surrounding environment(s), i.e. seawater in the case of unsedimented mid-ocean ridges like the EPR. The microorganisms colonizing basalt surfaces, however, are distinct from the deep ocean 
ecosystem in terms of: 1 . biomass - cell densities are several orders of magnitude higher on lavas, 2. biomass composition - bacterial cells are more prevalent on basalt surfaces compared to equal proportions of Bacteria and Archaea in deep seawater, and 3. species diversity - rock-hosted bacterial communities are significantly more diverse than in deep seawater. The observed rise in biomass on increasingly altered basalts provides additional support for our hypothesis. The most altered sample was erupted at least several thousand years ago, while the least altered lava was erupted relatively recently. Even a more conservative estimate of turnover times could not account for a 3 fold increase in biomass if seawater components were providing the bulk of the energy.

In addition to basalt oxidation reactions, other potential energy sources capable of sustaining microbial life exist in this bare-rock environment. The magmatic/hydrothermal input of hydrogen, methane, and hydrogen sulfide into the system could support microbial growth through a variety of metabolisms, even at some distance to the vent fields. Dissolved organic carbon (DOC) in seawater or hydrothermal fluids (Lang et al., 2006) could also provide energy for slow-growing heterotrophic microorganisms at the seafloor. It is suggested, however, that most DOC in the deep-sea is recalcitrant to microbial oxidation (Barber, 1968), but this remains largely under examined. Finally, the complexity observed in the basalt-hosted communities is not necessarily a reflection of diversity purely within the chemolithoautotrophic foundation of the biotope. Rather, this complexity potentially results from the establishment of micro-environments within/on rock cavities and surfaces during alteration processes, secondary mineral precipitation, and biofilm formation. The niches created would allow 
for a wide variety of chemical and redox reactions at close spatial scales, including organic, anaerobic or reduction reactions that could support heterotrophic and mixotrophic communities, all which expectedly contribute to the observed diversity, abundance, and dynamics of the basalt-hosted biotope.

\subsection{Methods and Materials}

\subsubsection{Sample collection.}

Samples were collected from the East Pacific Rise, $9^{\circ} \mathrm{N}$ (Appendix 2 Fig. A2.1) during research cruises AT11-7 and AT11-20 on the R/V Atlantis in February and November, 2004, respectively. The DSV Alvin was used to obtain lava from the seafloor from the axial summit trough up to approximate $3 \mathrm{~km}$ off-axis on the ridge flanks at a water depth of $\sim 2500-2700 \mathrm{~m}$. Rock samples were placed in bioboxes containing sterile freshwater, allowed to fill with ambient bottom seawater, sealed to minimize contamination from the upper water column, and brought to the surface for immediate processing. Ambient deep seawater was collected at the seafloor during sampling operations using Niskin bottles.

\subsubsection{DNA extraction and sequencing.}

Samples were crushed to mm-sized fractions using a sterile mortar and pestle immediately following sample collection. These subsamples were either processed at once or were stored at $-80^{\circ} \mathrm{C}$ for less than 24 hours until DNA extractions could be performed. Total community DNA was extracted directly after collection from $\sim 1 \mathrm{~g}$ 
crushed basalt using the Ultraclean soil DNA kit (MoBio Laboratories Inc.) following a slightly modified manufacturer's protocol. Prior to bead beating, the mixture was incubated for 10 minutes at $70^{\circ} \mathrm{C}$ after which $200 \mu \mathrm{g}$ of polyadenylic acid (poly A) was added (Webster et al., 2003). For the deep seawater sample, $50 \mathrm{~mL}$ of fluid was filtered $(0.2-\mu \mathrm{m}$-pore-size $)$, and extractions were performed directly on the filter using the protocol outlined above. The 16S rRNA region of environmental DNA was amplified using the bacteria-specific primer 8F (5'-AGAGTTTGATCCTGGCTCAG-3') and universal primer 1492R (5'-GGTTACCTTGTTACGACTT-3'). Negative controls were executed at all steps beginning with the DNA extractions (reagents only).

Positively amplified PCR products were purified using the QIAquick ${ }^{\circledR}$ nucleotide removal kit (Qiagen) following the manufacturer's instructions for the cleanup of nonradiogenic samples using centrifugation. Negative controls yielded no amplification products, verifying sterile technique. Purified amplification products were cloned using the $\mathrm{pCR} \otimes 2.1-\mathrm{TOPO}{ }^{\circledR}$ vector (Invitrogen), and clone libraries were constructed as described in a previous publication (Rogers et al., 2003). Plasmid extractions were done by alkaline lysis at the Keck Facility of the Josephine Bay Paul Center (JBPC), Marine Biological Laboratories (MBL). Sequencing reactions were carried out using the primer set M13F (5'- GTAAAACGACGGCCAG) and M13 R (5'-CAGGAAACAGCTATGAC) with the ABI v 3.1 BigDye terminator sequencing kit (Applied Biosystems). Reactions were analyzed on an ABI3730XL capillary sequencer at the JBPC.

\subsubsection{Phylogenetic analysis}


Sequence reads were edited and assembled using an automated pipeline, straw, from the JBPC at the Marine Biological Laboratories (MBL), Woods Hole, MA (http:// jbpc.mbl.edu/computing-seqinformatics.html). The automated pipeline utilizes the programs: phred (base-calling), cross-match (vector screening), and phrap (contig assembly). Finalized sequences were aligned using the ARB software package (http://www.arb-home.de/) and the most recent 16S ARB database (corrected version of ssujan04.arb). Chimeric sequences were identified using the program Bellerophon (Huber et al., 2004). Putative chimeras were checked against the sequences of several known sequences using ARB as well as with the chimera detection program (Cole et al., 2003) so only true chimeric sequences were removed.

Clone libraries of lava samples ranging from fresh (little sign of oxidative alteration) to slightly altered (Fe-oxides, Mn-oxides, palagonite, clays evident upon examination) were selected for further phylogenetic analyses in this study. Rather than treat each library individually, the sequences from all five lava samples were pooled to represent a single environment for further analyses. In order to determine phylogenetic affiliation, sequence data were submitted to the BLAST (Basic Local Alignment Search Tool) program to be compared against the GenBank 16S rRNA database (Altschul et al., 1997). The computer program (http://www.plantpath.wisc.edu/fac/joh/DOTUR.html; (Schloss and Handelsman, 2005)) was used to construct rarefaction curves and calculated species richness estimators. A $97 \%$ sequence similarity definition was selected to compare sequences at the species level for both rarefaction and diversity estimates. Sequences from the hydrothermal vent 
in-situ growth chamber (Reysenbach et al., 2000) were accessed from GenBank. The Minnesota farm soil sequences were kindly provided with permissions from the authors (Tringe et al., 2005). Sequence data from the Sargasso Sea (Venter et al., 2004) was previously analyzed by Schloss and Handelsman (2005) in which they extracted 16S rRNA oligonucleotides from the metagenome study and performed DOTUR analyses, was downloaded from the DOTUR website (address above).

\subsubsection{CARD-FISH and Microscopy}

Upon retrieval, rock samples for CARD-FISH analyses were immediately fixed in 4\% (wt/vol) paraformaldehyde for several hours, rinsed, and stored in PBS:ethanol $(1: 1)$ at $-20^{\circ} \mathrm{C}$ for subsequent analysis. Hybridizations were performed on mm-sized basalt chips using previously outlined protocols (Pernthaler et al., 2002; Sekar et al., 2003) that have been adapted for rock fragments. Successive treatments of lysozyme and achromopeptidase were used to permeabilize the cell walls so that both archaea and bacteria could be sufficiently represented by CARD-FISH analyses. All hybridizations were performed at $35^{\circ} \mathrm{C}$ with $55 \%$ formamide concentration. Bacteria or Archaea were targeted using horseradish peroxidase (HRP)-linked oligonucleotide probes EUB338-I, II, and -III (Daims et al., 1999) or ARCH915 (Raskin et al., 1994), respectively, with Cy3-tyramides synthesized by Biomers.net (Ulm, Germany). Dual hybridizations for Bacteria and Archaea were not performed on the same basalt chip, but were performed individually on subsets for each sample. SYBR Green I DNA stain (Invitrogen) was used as a counterstain. An LSM510-META laser scanning confocal microscope (Carl Zeiss, 
Germany) based on an Axioskop 2FS fixed-stage upright was used with a $\mathrm{HeNe}$ laser (543 nm wavelength) and an Ar ion laser (488 $\mathrm{nm}$ wavelength) at the MBL Central Microscopy Center to image cells directly on the rock surfaces by collecting dozens of vertical image slices (z-stacks). An Achroplan IR 63x/0.90W (water immersion)

objective was used for all imaging purposes. Very minimal to zero background fluorescence was observed in our samples. The LSM Image Browser version 3.5 (Zeiss) was used to create 3-dimensional image projections from the z-stacks. Adobe Photoshop was used for final image processing (cropping and adjusting contrast/brightness), which was minimal as to not affect the interpretation of the results.

A subset of the basaltic lava samples fixed in paraformaldehyde for CARD-FISH analyses was prepared for scanning electron microscopy (SEM). Samples were rinsed in sterile water to remove salts, dehydrated, and sputter coated with a gold/palladium mix. Images were obtained at the MBL Microscopy Center with a JEOL 840 SEM (JEOL Ltd., Japan) operated at $15 \mathrm{kV}$. Electronic images were processed and finalized in Adobe Photoshop.

\subsection{5 qPCR}

Real-time PCR (qPCR) assays were performed to assess the domain level microbial community structure of seafloor basalts. qPCR using the Taqman approach (Applied Biosystems, Foster City, CA) was used to quantify bacterial, archaeal, and total prokaryotic 16S rDNA gene copies, from which cell densities were approximated. 
Assays were performed on a subset of the genomic DNA extracted using the methods described above.

The qPCR assay to estimate Archaea used the primer set Arch349F (5'GYGCASCAGKCGMGAAW-3"), and Arch806R (5'-GGACTACVSGGGTATCTAAT3') with the TaqMan probe Arch516F ((6-FAM)-5'-TGYCAGCCGCCGCGG TAAHACCVGC-3'-(Iowa Black FQ)). To estimate total prokaryotes, the primer set Uni340F (5'-CCTACGGGRBGCASCAG-3') and Uni806R 5'-(GGACTACN NGGGTATCTAAT-3') with the TaqMan probe Uni516F ((6-FAM)-5'-TGYC AGCMGCCGCGGTAAHACVNRS-3'-(Iowa Black FQ)) was used. Both the archaeal and total prokaryote primer/probe sets were previously designed and tested by Takai and Horikoshi (2000). These primers and probes were synthesized by Integrated DNA Technologies (IDT, Coralville, IA). Primer/probe sets designed by Nadkarni et al. (2002) were used for bacterial DNA quantification. Primers 331F (5'TCCTACGGGAGGCAGCAGT-3) and 797R (5'-GGACTACCAGGGTATCTAAT CCTGTT-3') used in conjunction with TaqMan probe, (6-FAM)-5'CGTATTACCGCGGCTGCTGGCAC-3'-(TAMRA) were purchased from Thermo Electron Corporation.

All qPCR assays were performed on an ABI Prism 7000 Sequence Detection System (Applied Biosystems) in 96-well optical grade plates. Each PCR reaction contained the following reagents at final concentrations: 1x TaqMan Universal PCR Master Mix (Applied Biosystems) , 1 unit Platinum Taq (Invitrogen), 1x ROX (Invitrogen), $800 \mathrm{nM}$ forward primer, $800 \mathrm{nM}$ reverse primer, $200 \mathrm{nM}$ Taqman probe, 
template ( $3 \mu \mathrm{L}$ regardless of concentration), and molecular grade water to bring the reaction to a total volume of $30 \mu \mathrm{L}$. Extra ROX fluorescent dye and Platinum Taq (final concentrations shown above) had been added to the concentrations already present in the TaqMan Universal PCR Master Mix to improve the signal:noise ratio. The amplification protocol was similar for all assays, except for the annealing/extension temperature, which depended on the primer/probe set being used. The reaction conditions for amplification were $50^{\circ} \mathrm{C}$ for $2 \mathrm{~min}, 95^{\circ} \mathrm{C}$ for $10 \mathrm{~min}$, and 45 cycles of $95^{\circ} \mathrm{C}$ for $15 \mathrm{sec}$ and either $57^{\circ} \mathrm{C}$ (total prokaryotes) or $59^{\circ} \mathrm{C}$ (archaea and bacteria) for $3 \mathrm{~min}$. All reactions (standards, samples, and blanks) were performed in triplicate, and all PCR runs include a no template control.

Standard curves (regression line of threshold cycle $(\mathrm{Ct})$ versus log concentration) for quantifying gene copies were determined using a mixture of archaeal and bacterial plasmid DNA of known concentration $(10 \mathrm{ng} / \mu \mathrm{L}$ each) orginally isolated (and cloned) from microbial mats at hydrothermal vents along the Mariana Island Arc (Davis and Moyer, 2005). For bacterial DNA quantification, serial dilutions $\left(1 \mathrm{X}-10^{-6} \mathrm{X}\right.$ concentration) of non-linearized plasmids were used to construct the standard curve, which was linear $\left(r^{2}=0.99\right)$ with a minimum detection concentration of $1.5 \times 10^{-5} \mathrm{ng}$ DNA reaction $^{-1}$ and an amplification efficiency (E) of 94\%. However when used for estimating archaeal and total prokaryotic DNA, the non-linearized plasmids had poor amplification efficiencies (70\% and $65 \%$ respectively) and were linear for a shorter range of the dilution series. For example, the detection limit of the prokaryotic DNA was $1.5 \times 10^{-3} \mathrm{ng}$ DNA reaction ${ }^{-1}$. To increase sensitivity and efficiency, purified PCR products of the 
initial bacterial and archaeal plasmids were attempted with the primer/probe sets. PCR efficiency and sensitivity both increased using the PCR products as standards to quantify Archaea $\left(\mathrm{E}=76 \%, \mathrm{r}^{2}=0.99, \mathrm{~min} .=1.5 \times 10^{-5} \mathrm{ng}\right.$ DNA reaction $\left.{ }^{-1}\right)$ and total prokaryotes $(\mathrm{E}$ $=85 \%, \mathrm{r}^{2}=0.99, \min .=1.5 \times 10^{-5}$ ng DNA reaction $\left.{ }^{-1}\right)$.

Serial dilutions of samples from $\left(1 \mathrm{X}-10^{-3} \mathrm{X}\right)$ were also performed to ensure that the PCR efficiency was similar to that of the standards. Most samples amplified with approximately the same efficiencies as the standards for each respective primer/probe set. However for many samples, the undiluted sample (1X dilution concentration) was the only measurable sample of the series. Sample DNA concentrations were calculated based on the line equation of the standards and the threshold cycle $(\mathrm{Ct})$ of each PCR reaction using the equation: Conc. (sample) $=(\mathrm{Ct}$ (sample) $-\mathrm{y}$ intercept (standards))/(slope (standards)). DNA concentrations were converted to gene copy number using the assumption that a single double-stranded DNA molecule has a mass of $660 \mathrm{~g} \mathrm{~mol}^{-1}$. A conversion factor of $4.1\left(16 \mathrm{~S}\right.$ rDNA gene copies cell $\left.{ }^{-1}\right)$ was used to determine bacterial and total prokaryote cell abundances, whereas 1.6 was used to convert archaeal gene copies to cell numbers (Klappenbach et al., 2001). Final DNA concentrations and cell densities were normalized to $1 \mathrm{~g}$ basalt, which was used for the initial genomic DNA extractions. Biomass (\% archaea or bacteria) was quantified by summing bacterial and archaeal cell abundances to obtain total cell numbers, rather than using cell numbers determined by the total prokaryote qPCR assay because this assay had a very poor efficiency when run with standards (potentially due to poor primer/probe design). 


\subsection{Acknowledgements}

The authors thank Dan Fornari, Maurice Tivey, and Hans Schouten for allowing C.M.S. and W.B. to participate in their research cruise AT11-7, Luis Kerr at the Central Microscope Facility at the MBL for instruction and guidance on the SEM and confocal microscope, Sheri Simmons for support and instruction on qPCR, and Erin Banning for instruction with CARD-FISH, and Edward Leadbetter for advice on the manuscript. This research was supported by a Ridge $2 \mathrm{~K}$ grant awarded to K.J.E. and W.B. and also in part by a Project Development Award from WWU's Office of Research and Sponsored Programs to C.L.M. 


\subsection{Figures}
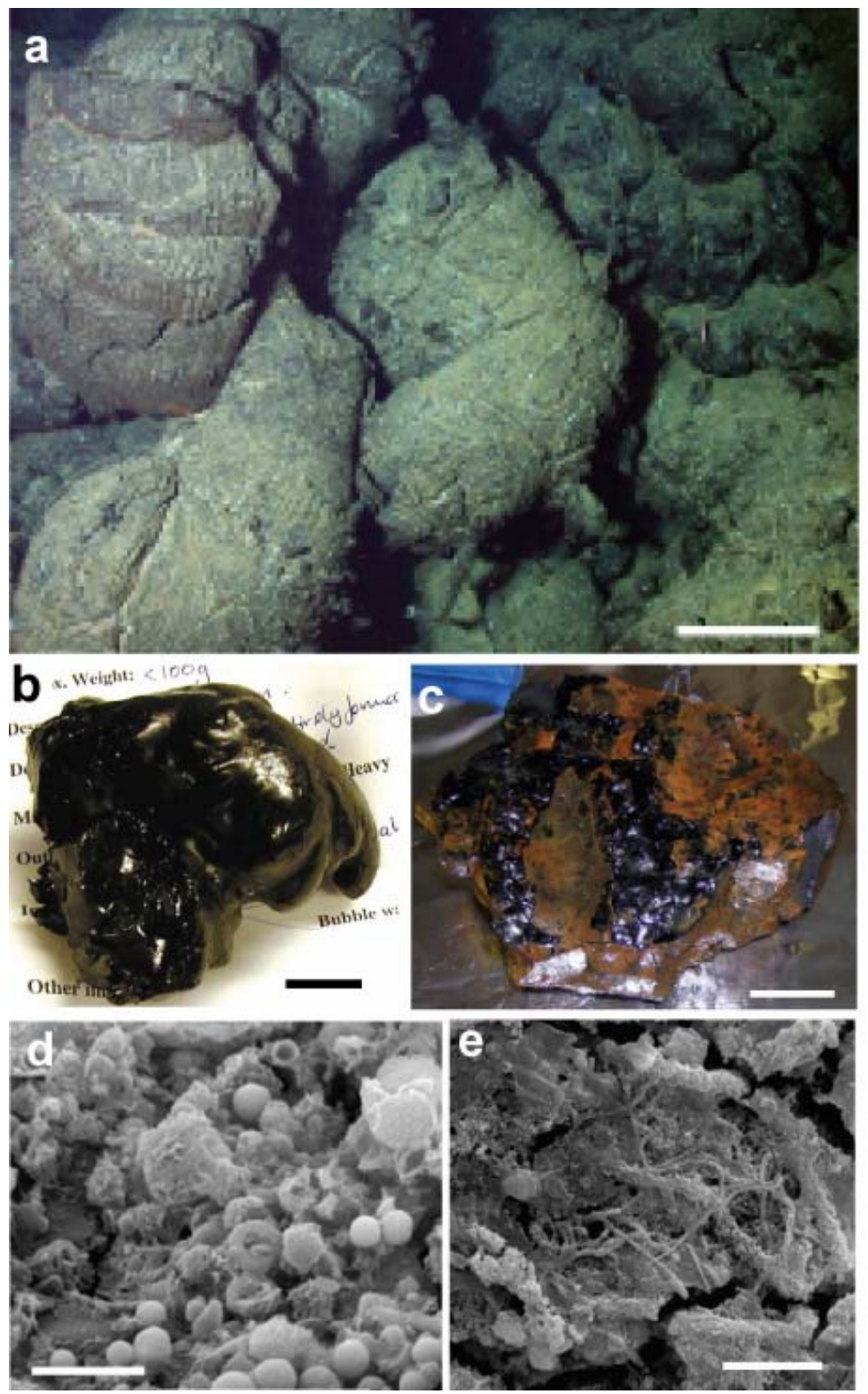

Figure 2.1. A highly diverse and abundant epi- and endo-lithic microbial community exists on basaltic lavas from the East Pacific Rise. a, The East Pacific Rise at $9^{\circ} \mathrm{N}$ is characterized by lava flows, such as pillow basalts shown here, directly exposed at the seafloor. Scale bar, $40 \mathrm{~cm}$. b,c, Photographs showing the range of volcanic samples used in this study from fresh and glassy (b) to more altered and oxide coated (c). Scale bars, 2 $\mathrm{cm}$ (b) and $4 \mathrm{~cm}$ (c). d,e, SEM images of different presumed cellular morphologies, such as coccoidal (d) and filamentous (e) structures that were observed on ferromanganese oxide encrusted and Fe-oxide-coated samples. Scale bars, $5 \mu \mathrm{m}$. 


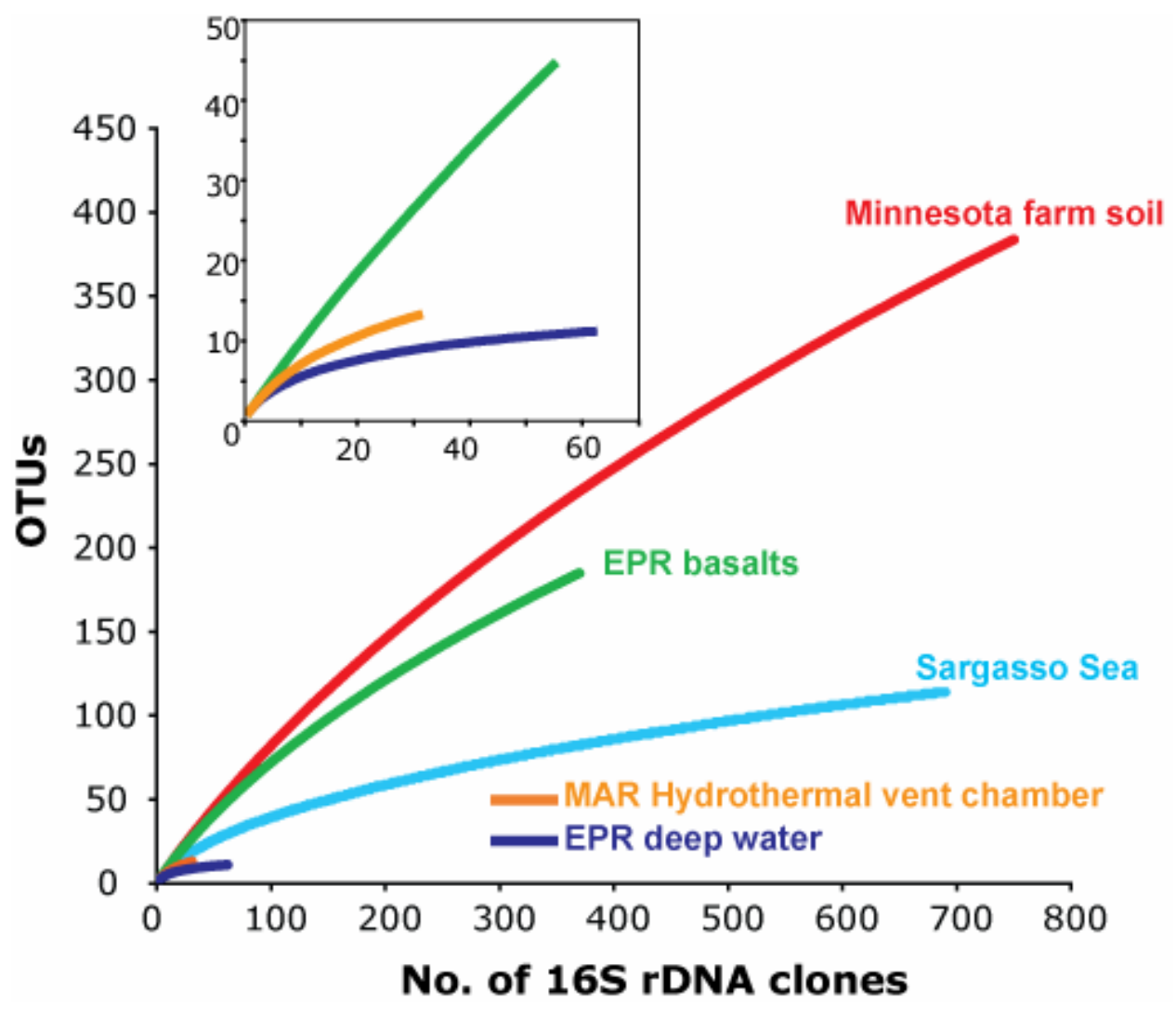

Figure 2.2. Relative bacterial diversity from several environmental studies shown through rarefaction analyses. The observed species diversity of Bacteria inhabiting seafloor lavas from the EPR is compared to the diversity found in clone library surveys from a farm soil, the Sargasso Sea, a MAR hydrothermal vent in situ growth chamber, and ambient bottom seawater from the EPR. Only approximately one-half of the entire rarefaction curve is shown for the farm soil survey for visualization purposes. The projection of this curve does change with increased sampling effort. Inset, close-up of rarefaction curves for the hydrothermal chimney and EPR deep seawater studies (libraries with fewer clones) relative to the EPR basalts survey. Each study is based on near fulllength $16 \mathrm{~S}$ rRNA gene sequences. OTUs are defined at a sequence similarity of $\geq 97 \%$. 
a

\section{EPR seafloor basalts}

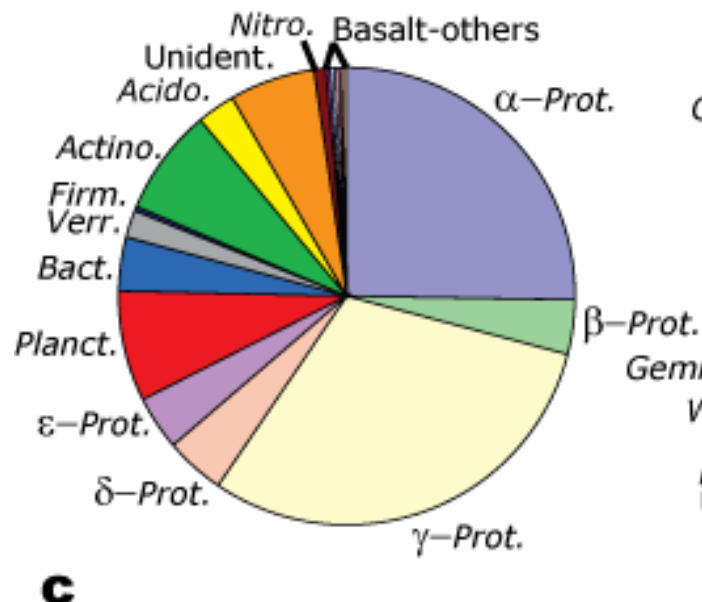

EPR deep seawater

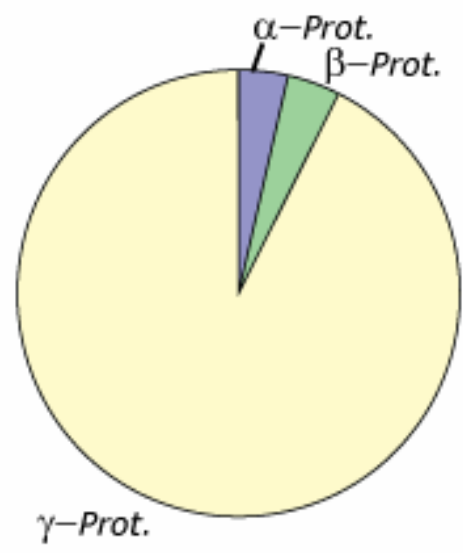

b

MN farm soil

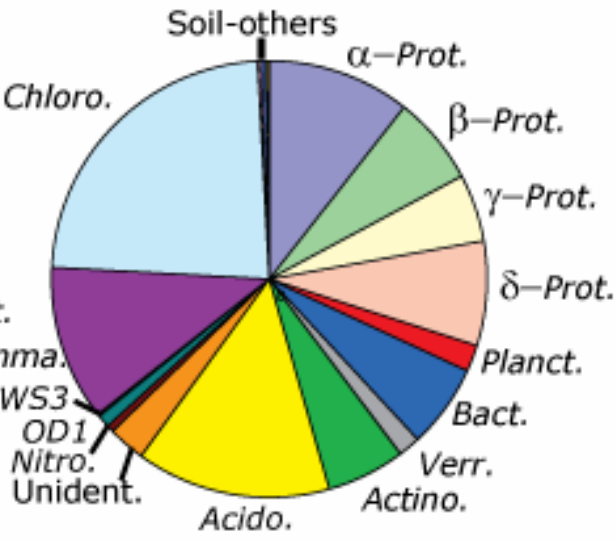

d

MAR hydrothermal vent in situ growth chamber

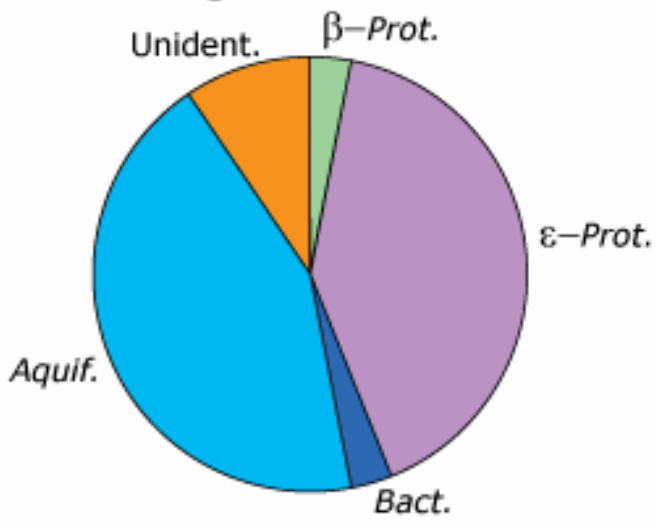

Figure 2.3. Phylogenetic identity of Bacteria from environmental studies. a-d, Distribution of bacterial 16S rRNA sequences into major taxonomic groups from the cumulative EPR basalt clone library (a), Minnesota farm soil (b), ambient bottom seawater from the EPR (c), and a MAR hydrothermal vent in situ growth chamber (d). Basalt-others $(<0.5 \%$ each): Gemmatimonadetes, Spirochaetes, Candidatus Scalindua brodae, and Cand. div. OP11. Soil-others ( $<0.5 \%$ each): Firmicutes, Cyanobacteria, Deinococcus-Thermus, Cand. divs. OP11, OP10, SPAM, WS6, TM7, and BRC1. Abbreviations: Prot., Proteobacteria; Bact., Bacteroidetes; Firm, Firmicutes; Verr., Verrucomicrobia; Planct., Planctomycetes; Chloro, Chloroflexi; Actino., Actinobacteria; Acido., Acidobacteria; Gemma., Gemmatimonadetes; Aquif., Aquificae; Nitro., Nitrospira; Unident., Unidentified. 


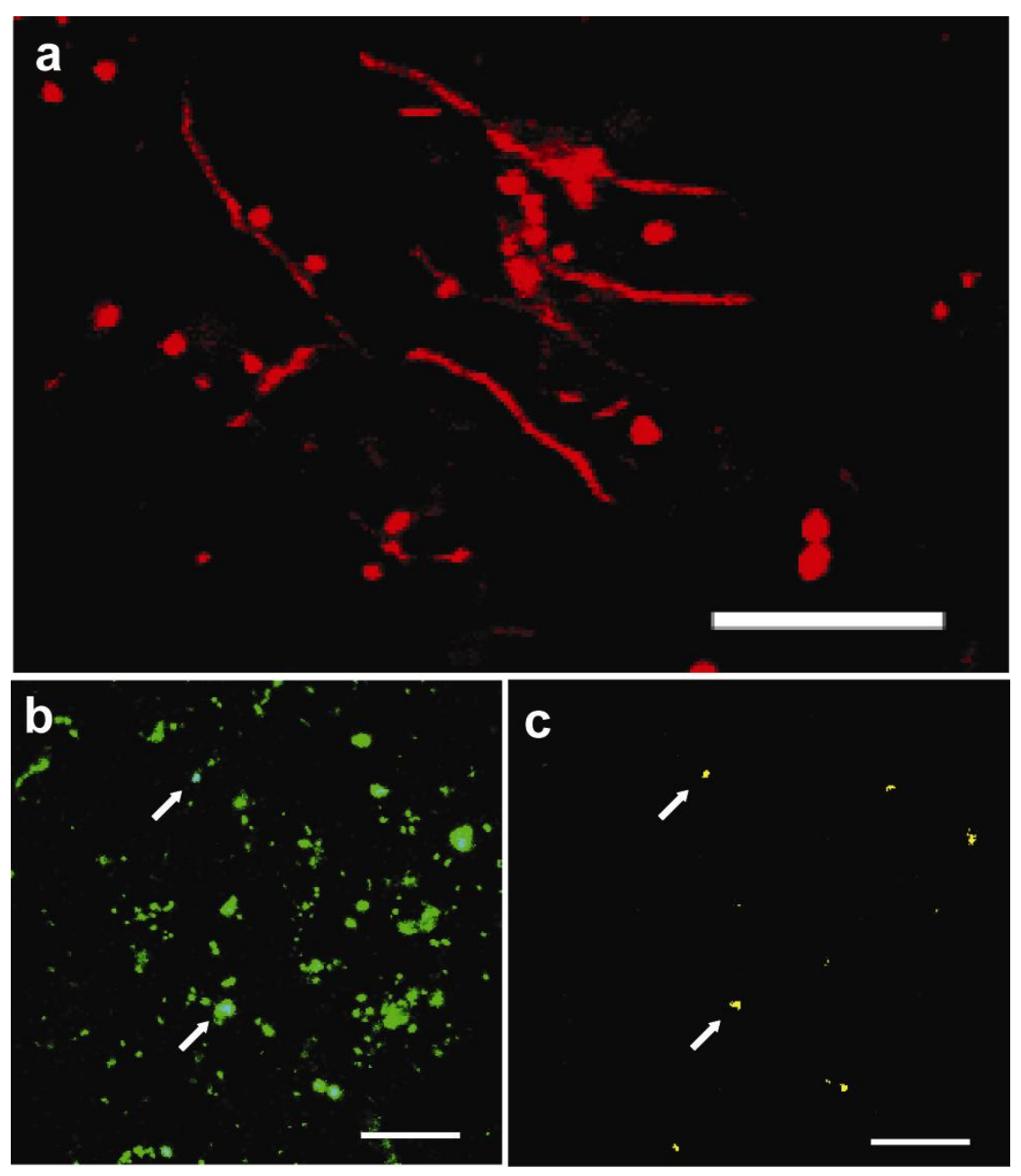

Figure 2.4. Phylogenetic identity of Bacteria from environmental studies. a,b,c, Confocal laser scanning micrographs depicting CARD-FISH on lava surfaces. Prokaryotic cells were hybridized with either probe EUB338(I-III) mix (a) or probe ARCH915 (b) to target Bacteria, or Archaea respectively. A variety of bacterial cell morphologies such as filaments, cocci, and rods were confirmed with hybridizations (a). Side-by-side comparisons of Archaea (b) versus total cells (c) reveals that Archaea account for only a small portion of the total cells. Total cells were identified with the general DNA stain SYBR Green. The arrows point to some cells that overlap in each frame. Scale bars, $10 \mu \mathrm{m}$ (a) and $20 \mu \mathrm{m}$ (b,c). 


\subsection{Appendix}

\section{Deep seawater analysis}

The distinctly low diversity of Bacteria and dominance of $\alpha$ - and $\gamma$ Proteobacteria phylotypes in the deep seawater clone library was also observed by Huber et al. $(2003 ; 2006)$ at the Juan de Fuca Ridge. These authors, however, also recovered $\delta$-Proteobacteria, Bacteroidetes, and Planctomycetes in their library. This suggests that our clone library may be missing a portion of the total diversity of Bacteria found in the deep seawater environment, possibly due to under-sampling or optimization of our DNA extraction and amplification protocols for rocks rather than fluids. A direct comparison of rarefaction plots for our EPR deep seawater clone library versus a cumulative ambient bottom seawater environment including our library in addition to the two Huber et al. libraries (Appendix 2 Fig. A2.2) indicates that the relative difference in species richness with respect to the EPR basalt richness remains the same. Both data sets

hence demonstrate that the phylogenetic diversity of ocean floor basalt is considerably greater than in surrounding seawater. 
Table A2.1 Richness estimators and diversity indices for Bacteria calculated using

DOTUR compared at a distance level of 0.03. Randomized subsets (OTU's $=370$ ) of the MN soil and Sargasso Sea microbial communities were also analyzed to directly compare the diversity to the EPR basalt community diversity with the same level of sample effort.

\begin{tabular}{|c|c|c|c|c|c|c|c|c|}
\hline & $\begin{array}{c}\text { \# of } \\
\text { clones }\end{array}$ & $\begin{array}{c}\text { OTU's } \\
\text { sampled }\end{array}$ & & $\mathrm{ACE}^{1}$ & Chao1 & $\begin{array}{l}\text { Jack- } \\
\text { knife }\end{array}$ & $\begin{array}{l}\text { Shannon } \\
\text {-Weaver }\end{array}$ & Simpson \\
\hline \multirow[t]{3}{*}{ MN soil } & 1749 & 655 & Index & 1441 & 1410 & 1894 & 5.84 & 0.007 \\
\hline & & & $\mathrm{LCl}^{2}$ & 1356 & 1223 & 1621 & 5.78 & $N P^{4}$ \\
\hline & & & $\mathrm{UCl}^{3}$ & 1536 & 1658 & 2166 & 5.91 & $N P$ \\
\hline \multirow[t]{3}{*}{ MN soil subset } & 370 & 241 & Index & 784 & 706 & 804 & 5.24 & 0.005 \\
\hline & & & $\mathrm{LCl}$ & 599 & 540 & 663 & 5.13 & $N P$ \\
\hline & & & $\mathrm{UCl}$ & 1064 & 965 & 945 & 5.34 & $N P$ \\
\hline \multirow[t]{3}{*}{ EPR Basalts } & 370 & 185 & Index & 487 & 440 & 567 & 4.82 & 0.011 \\
\hline & & & $\mathrm{LCl}$ & 380 & 338 & 445 & 4.7 & $N P$ \\
\hline & & & $\mathrm{UCl}$ & 609 & 609 & 688 & 4.93 & $N P$ \\
\hline \multirow[t]{3}{*}{ Sargasso Sea } & 692 & 140 & Index & 205 & 198 & 214 & NC & 0.06 \\
\hline & & & $L C I$ & $N C^{4}$ & 187 & NC & NC & $N P$ \\
\hline & & & $\mathrm{UCl}$ & NC & 211 & NC & NC & $N P$ \\
\hline Sargasso Sea & 370 & 88 & Index & 180 & 146 & 145 & $\mathrm{NC}$ & 0.07 \\
\hline \multirow{2}{*}{ subset } & & & $\mathrm{LCl}$ & $N C$ & 137 & $N C$ & $N C$ & $N P$ \\
\hline & & & UCl & $N C$ & 156 & NC & $N C$ & $N P$ \\
\hline Hydrothermal vent & 31 & 13 & Index & 20 & 16 & 19 & 2.3 & 0.095 \\
\hline \multirow[t]{2}{*}{ cap } & & & $\mathrm{LCl}$ & 15 & 14 & 12 & 2 & $N P$ \\
\hline & & & UCl & 43 & 31 & 26 & 2.6 & $N P$ \\
\hline \multirow[t]{3}{*}{ Deep seawater } & 62 & 8 & Index & 14 & 12 & 14 & 1.91 & 0.19 \\
\hline & & & $\mathrm{LCl}$ & 11 & 11 & 9 & 1.67 & $N P$ \\
\hline & & & $\mathrm{UCl}$ & 27 & 22 & 19 & 2.14 & NP \\
\hline
\end{tabular}

Abundance-based Coverage Estimator

${ }^{2}$ Lower bound of $95 \%$ confidence interval

${ }^{3}$ Upper bound of $95 \%$ confidence interval

${ }^{4}$ Not possible to calculate

${ }^{5}$ Could not be calculated 
Table A2.2. qPCR results showing the proportion of Bacteria and Archaea extracted from basalt samples ranging from fresh to moderately altered. Cell concentrations were determined using the approximations of 4.1 or 1.6 bacterial or archaeal cells per gene copy, respectively.

\begin{tabular}{|c|c|c|c|c|c|c|}
\hline \multirow[b]{2}{*}{ Sample } & \multicolumn{2}{|c|}{ 16S Gene Copies g ${ }^{-1}$ Basalt } & \multicolumn{2}{|c|}{ Cells g ${ }^{-1}$ Basalt } & \multirow{2}{*}{$\begin{array}{c}\% \\
\text { Bac. }\end{array}$} & \multirow{2}{*}{$\begin{array}{c}\% \\
\text { Arc. }\end{array}$} \\
\hline & Bacteria & Archaea & Bacteria & Archaea & & \\
\hline Fresh, glassy & $1.13 \pm 0.09 \times 10^{7}$ & $6.09 \pm 0.71 \times 10^{5}$ & $2.76 \pm 0.23 \times 10^{6}$ & $3.80 \pm 0.45 \times 10^{5}$ & 87.87 & 12.13 \\
\hline $\begin{array}{l}\text { Slight } \\
\text { alteration }\end{array}$ & $2.36 \pm 0.18 \times 10^{8}$ & $6.69 \pm 0.23 \times 10^{6}$ & $5.77 \pm 0.43 \times 10^{7}$ & $4.18 \pm 0.15 \times 10^{6}$ & 93.23 & 6.77 \\
\hline $\begin{array}{l}\text { Moderate } \\
\text { alteration }\end{array}$ & $5.70 \pm 0.42 \times 10^{9}$ & $1.21 \pm 0.36 \times 10^{8}$ & $1.39 \pm 0.10 \times 10^{9}$ & $7.54 \pm 2.28 \times 10^{7}$ & 94.85 & 5.15 \\
\hline
\end{tabular}




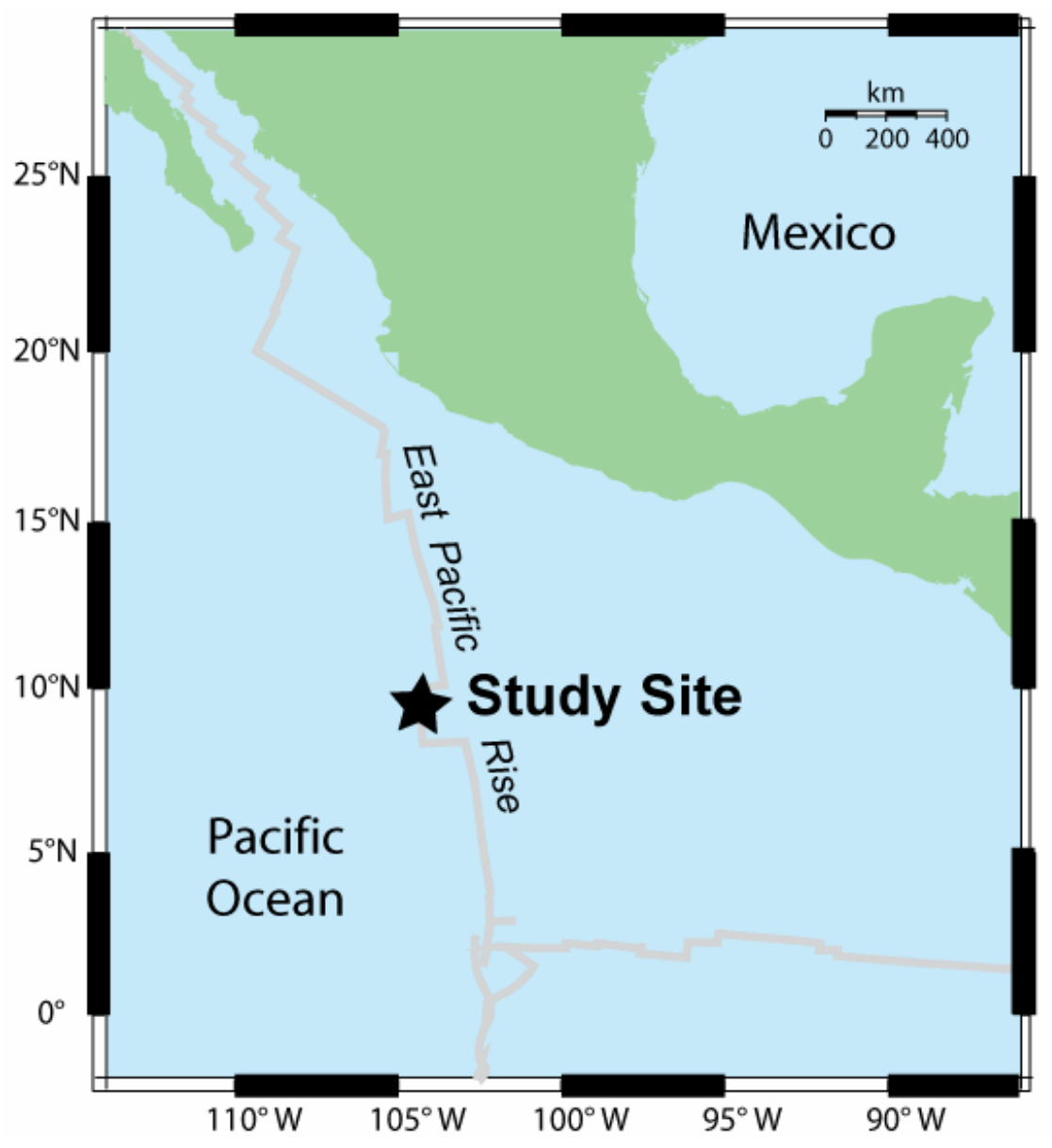

Figure A2.1. Map of the equatorial Eastern Pacific Ocean showing the location of the study site where seafloor lavas were collected. Basalt samples were collected on and near the ridge axis (gray lines) up to $3 \mathrm{~km}$ off-axis between approximately $9^{\circ} 28^{\prime} \mathrm{N}$ and $9^{\circ} 50^{\prime} \mathrm{N}$ 


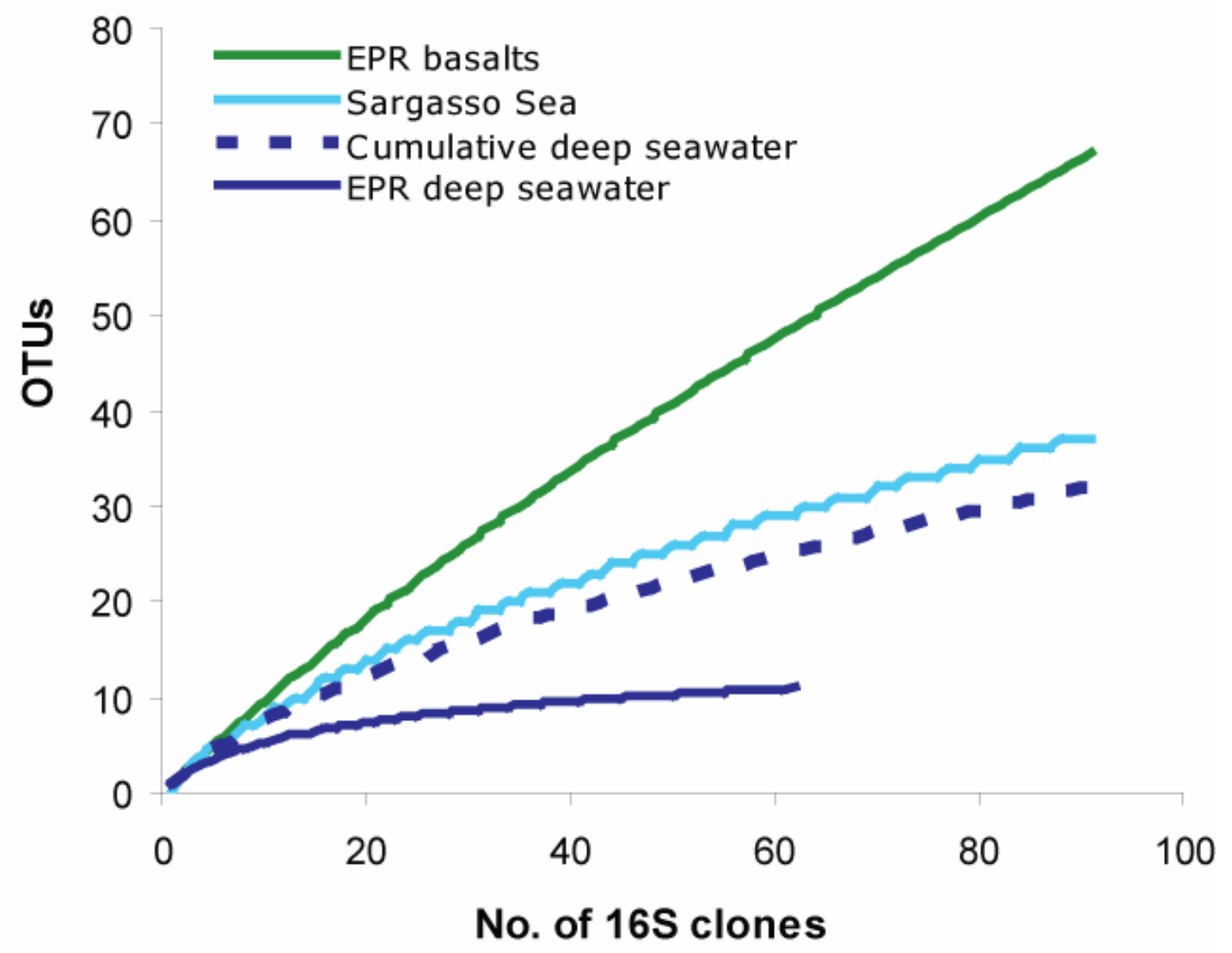

Figure A2.2. Rarefaction analyses comparing the diversity between EPR deep seawater and cumulative deep seawater (combination of clone libraries from 3 different studies including EPR deep seawater and two studies by Huber et al. (2003; 2006). OTU's are defined at a distance level of 0.03 as assigned by DOTUR. Analyses show that the addition of clones from the Huber et al. deep seawater libraries increases species diversity approximately equal to that of Sargasso Sea surface waters. However, the bacterial diversity found in deep seawater relative to that found in EPR basalt is still the same. 


\subsection{References}

Altschul, S.F. et al., 1997. Gapped BLAST and PSI-blast: a new generation of protein database search programs. Nucl. Acids Res., 25: 3389-3402.

Bach, W. and Edwards, K.J., 2003. Iron and sulfide oxidation within the basaltic ocean crust: Implications for chemolithoautotrophic microbial biomass production. Geochim. Cosmochim. Acta, 67(20): 3871-3887.

Barber, R.T., 1968. Dissolved organic carbon from deep water resists microbial oxidation. Nature, 220(274-275).

Biddle, J.F. et al., 2006. Heterotrophic archaea dominate sedimentary subsurface ecosystems off Peru. Proc. Natl. Acad. Sci., 103(10): 3846-3851.

Cole, J.R. et al., 2003. The ribosomal database project (RDP-II): previewing a new autoaligner that allows regular updates and the new prokaryotic taxonomy. Nucl. Acids Res., 31(1): 442-443.

Cowen, J.P. et al., 2003. Fluids from aging ocean crust that support microbial life. Science, 299(5603): 120-123.

Daims, H., Bruhl, A., Amann, R., Schleifer, K.-H. and Wagner, M., 1999. The domainspecific probe EUB338 is insufficient for the detection of all Bacteria: development and evaluation of a more comprehensive probe set. Syst. Appl. Microbiol., 22: 434-444.

Davis, R.E. and Moyer, C.L., 2005. Extreme spatial variability in microbial mat communities from submarine hydrothermal vents located at multiple volcanoes along the Mariana Island Arc. Abstr. Fall Mtg. Eos. Trans. AGU, 86(V51C-1509).

D'Hondt, S. et al., 2004. Distributions of microbial activities in deep subseafloor sediments. Science, 306: 2216-2221.

Edwards, K.J., Rogers, D.R., Wirsen, C.O. and McCollom, T.M., 2003. Isolation and characterization of novel psychrophilic, neutrophilic, Fe-oxidizing, chemolithoautotrophic alpha- and gamma-proteobacteria from the deep sea. Appl. Environ. Microbiol., 69(5): 2906-2913.

Hong, S.-H., Bunge, J., Jeon, S.-O. and Epstein, S.S., 2006. Predicting microbial species richness. Proc. Natl. Acad. Sci., 203(1): 117-122.

Huber, J., Butterfield, D. and Baross, J., 2003. Bacterial diversity in a subseafloor habitat following a deep-sea volcanic eruption. FEMS Microbiol. Ecol., 43(3): 393-409.

Huber, J.A., Johnson, H.P., Butterfield, D.A. and Baross, J.A., 2006. Microbial life in ridge flank crustal fluids. Appl. Environ. Microbiol., 8(1): 88-99.

Huber, T., Faulkner, G. and Hugenholtz, P., 2004. Bellerophon: a program to detect chimeric sequences in multiple sequence alignments. Bioinformatics, 20(14): 2317-2319.

Hughes, J.B. and Hellmann, J.J., 2005. The application of rarefaction techniques to molecular inventories of microbial diversity. Methods Enzymol., 397: 292-308.

Hughes, J.B., Hellmann, J.J., Ricketts, T.H. and Bohannan, B.J.M., 2001. Counting the uncountable: statistical approaches to estimating microbial diversity. Appl. Environ. Microbiol., 67(10): 4399-4406. 
Karner, M.B., DeLong, E.F. and Karl, D.M., 2001. Archaeal dominance in the mesopelagic zone of the Pacific Ocean. Nature, 409: 507-510.

Klappenbach, J.L., Saxman, P.R., Cole, J.R. and Schmidt, T.M., 2001. rrndb: the ribosomal RNA operon copy number database. Nucl. Acids Res., 29: 181-184.

Lang, S.Q., Butterfield, D.A., Lilley, M.D., Johnson, H.P. and Hedges, J.I., 2006. Dissolved organic carbon in ridge-axis and ridge-flank hydrothermal systems. Geochim. Cosmochim. Acta, 70: 3830-3842.

Moyer, C.L., Tiedge, J.M., Dobbs, F.C. and Karl, D.M., 1998. Diversity of deep-sea hydrothermal vent Archaea from Loihi Seamount, Hawaii. Deep-Sea Res. II, 45: 303-317.

Nadkarni, M.A., Martin, F.E., Jacques, N.A. and Hunter, N., 2002. Determination of bacterial load by real-time PCR using a broad-range (universal) probe and primers set. Microbiology, 148: 257-266.

Pernthaler, A., Pernthaler, J. and Amann, R., 2002. Fluorescence in situ hybridization and catalyzed reporter deposition for the identification of marine bacteria. Appl. Environ. Microbiol., 2002(68): 6.

Raskin, L., Stromley, J.M., Rittmann, B.E. and Stahl, D.A., 1994. Group-specific 16S ribosomal-RNA hybridization probes to describe natural communities of methanogens. Appl. Environ. Microbiol., 60(1232-1240): 1232-1240.

Ravenschlag, K., Sahm, K., Pernthaler, J. and Amann, R., 1999. High bacterial diversity in permanently cold marine sediments. Appl. Environ. Microbiol., 65(9): 39823989.

Reysenbach, A.-L., Longnecker, K. and Kirshtein, J., 2000. Novel bacterial and archaeal lineages from an in situ growth chamber deployed at a Mid-Atlantic ridge hydrothermal vent. Appl. Environ. Microbiol., 66(9): 3798-3806.

Rogers, D.R., Santelli, C.M. and Edwards, K.J., 2003. Geomicrobiology of deep-sea deposits: estimating community diversity from low-temperature seafloor rocks and minerals. Geobiology, 1: 109-117.

Schloss, P.D. and Handelsman, J., 2005. Introducing DOTUR, a computer program for defining operational taxonomic units and estimating species richness. Appl. Environ. Microbiol., 71(3): 1501-1506.

Schloss, P.D. and Handelsman, J., 2006. Introducing SONS, a tool for operational taxonomic unit-based comparisons of microbial community memberships and structures. Appl. Environ. Microbiol., 72(10): 6773-6779.

Schrenk, M.O., Kelley, D.S., Delaney, J.R. and Baross, J.A., 2003. Incidence and diversity of microorganisms within the walls of an active deep-sea sulfide chimney. Appl. Environ. Microbiol., 69(6): 3580-3592.

Sekar, R. et al., 2003. An improved protocol for quantification of freshwater Actinobacteria by fluorescence in situ hybridization. Appl. Environ. Microbiol., 69(5): 2928-2935.

Takai, K. and Horikoshi, K., 2000. Rapid detection and quantification of members of the archaeal community by quantitative PCR using fluorogenic probes. Appl. Environ. Microbiol., 66(11): 5066-5072. 
Torsvik, V., Goksoyr, J. and Daae, F.L., 1990. High diversity in DNA of soil bacteria. Appl. Environ. Microbiol., 65(3): 782-787.

Tringe, S.G. et al., 2005. Comparative metagenomics of microbial communities. Science, 22: 554-557.

Venter, J.C. et al., 2004. Environmental genome shotgun sequencing of the Sargasso Sea. Science, 304: 66-74.

Webster, G., Newberry, C.J., Fry, J.C. and Weightman, A.J., 2003. Assessment of bacterial community structure in the deep sub-seafloor biosphere by $16 \mathrm{~S}$ rDNAbased techniques: a cautionary tale. Journal of Microbiological Methods, 55: 155164.

Whitman, W.B., Coleman, D.C. and Wiebe, W.J., 1998. Prokaryotes: The unseen majority. Proc. Natl. Acad. Sci., 95: 6578-6583. 


\title{
Chapter 3. The diversity and abundance of bacteria inhabiting seafloor lavas positively correlate with the extent of rock alteration
}

\author{
Cara M. Santelli ${ }^{1}$, Virginia P. Edgcomb ${ }^{2}$, Wolfgang Bach ${ }^{3}$, Katrina J. Edwards ${ }^{4 *}$ \\ ${ }^{I}$ MIT/WHOI Joint Program in Oceanography and Ocean Engineering \\ ${ }^{2}$ Geology and Geophysics Department, Woods Hole Oceanographic Institution, MS \#52, \\ Woods Hole, Massachusetts, 02543, USA \\ ${ }^{3}$ Fachbereich Geowissenschaften, Universität Bremen, Postfach 3304 40, D-28334 \\ Bremen, Germany \\ ${ }^{4 *}$ Geomicrobiology Group, Department of Biological Sciences, Marine Environmental \\ Biology, University of Southern California, 3616 Trousdale Blvd, Los Angeles, CA, USA
}

\begin{abstract}
Young, basaltic ocean crust near mid-ocean ridges (MORs) presents the potential to harbor a vast, endolithic microbial ecosystem. Geochemical energy released during basalt alteration reactions could support chemosynthetic communities, which we hypothesize represent the trophic base for the ocean crust biotope. To establish the relationship between microbial growth and basalt alteration processes, we compare the phylogenetic diversity, richness, and abundance of bacteria existing in several young, seafloor lavas from the East Pacific Rise (EPR) at $\sim 9^{\circ} \mathrm{N}$ that are variably affected by oxidative seawater alteration. The results of 16S rRNA gene analyses and real-time, quantitative polymerase chain reaction (qPCR) measurements show that the abundance of prokaryotic communities, dominated by the bacterial domain, directly correlates with the extent of rock alteration - the oldest, most altered basalt harbors the greatest microbial
\end{abstract}


biomass. The species richness and the bacterial community overlap and structure relative to alteration state is less explicit, however the diversity and community complexity broadly corresponds to the basalt characteristics (type of alteration products and general alteration state). Furthermore, phylogenetic analyses suggest that the seafloor lavas host a variety of species that may be contributing to the geochemical cycling of S, Fe, Mn, C, and $\mathrm{N}$ in the deep-sea environment.

\subsection{Introduction}

Ocean crust near mid-ocean ridges (MORs) is composed largely of glassy, basaltic lava flows that are highly enriched in reduced species of elements such as $\mathrm{Fe}, \mathrm{S}$, and $\mathrm{Mn}$. Upon exposure to oxygenated deep seawater, a variety of oxidation and hydration (hydrogen generating) reactions occur on the surfaces and within the fractures and void spaces of the basalt, particularly in the glassy portions (Alt, 1995). These lowtemperature rock alteration processes dominate sparsely sedimented, seafloor exposed MOR environments and young ridge flank crust (an estimated 1,000,000 $\mathrm{km}^{2}$ of the Earth's surface), and extend several hundred meters into the subsurface where large volumes of seawater circulate within this highly fractured, permeable basalt aquifer. Additionally at MORs, hydrothermal fluids rich in reduced compounds such as $\mathrm{H}_{2} \mathrm{~S}, \mathrm{H}_{2}$, and $\mathrm{CH}_{4}$ are continually flowing out of the crust. Microbial communities could catalyze redox reactions of species/compounds within or emanating from this rocky habitat for metabolic growth, rendering the MOR system the potentially largest, continuous endolithic habitat on the planet. 
Indeed, a number of studies have recently demonstrated the subsistence of diverse and prolific microbial communities on young, basaltic ocean crust (Chapter 2, Lysnes et al., 2004a) and in fluids emanating from the crust (Cowen et al., 2003; Huber et al., 2003; Huber et al., 2006). Phylogenetic analyses (Chapter 2) show that seafloor lavas from the East Pacific Rise (EPR) harbor substantially more bacterial diversity in relation to several other comparable ocean habitat studies, e.g. ambient bottom seawater at the EPR, the Sargasso Sea (Venter et al., 2004), and a hydrothermal vent in situ growth chamber from the Mid-Atlantic Ridge (Reysenbach et al., 2000). In fact, the bacterial richness of the ocean crust biotope is comparable to that recovered in a terrestrial soil study (Tringe et al., 2005), an environment that has generally been accepted to harbor the greatest phylogenetic diversity on Earth (Moyer et al., 1998; Torsvik et al., 1990). Though the energetic underpinnings of this biotope are not fully elucidated, it has been suggested through both observation (Chapter 2) and thermodynamic/bioenergetic calculations (Bach and Edwards, 2003) that chemolithoautotrophy could potentially serve as the trophic base for ecosystems in this oligotrophic, bare-rock environment - the growth of chemolithoautrophic microorganisms could be supported by the release of chemical energy during fluid-rock alteration reactions. Furthermore, it has been demonstrated that chemosynthetic communities represent the trophic base in a number of terrestrial subsurface environments (Edwards et al., 2000; Lovley and Chapelle, 1995; Pedersen et al., 1997), but this potential has only rarely been empirically examined in the deep sea (Eberhard et al., 1995; Wirsen et al., 1993). 
Here we examine the changes in phylogenetic diversity and abundance of bacteria inhabiting young, basaltic seafloor lavas from the $9^{\circ} \mathrm{N}$ region of the EPR, a portion of the MOR system characterized by a fast spreading rate $\left(\sim 11 \mathrm{~cm} \mathrm{yr}^{-1}\right.$ full spreading rate $)$ (Carbotte and Macdonald, 1992), active hydrothermal venting, and unsedimented seafloor lava flows directly exposed to seawater. In an initial study of this biotope (Chapter 2), we demonstrated that the EPR basalts are dominated by a phylogenetically diverse group of actively metabolizing bacterial communities. In this study, we focus on the phylogenetic diversity (near full-length 16S rRNA gene sequence analyses) and abundance (quantitative PCR analyses) of bacteria detected in each variably altered seafloor lava sample. We also compare the phylotype richness, membership, and community structure for each basalt sample using statistical tools that enable us to determine patterns of association between taxa, microbial abundances, and rock alteration. Our end goal is to discern the contribution of microbial activity to alteration processes and geochemical cycling in this rocky habitat.

\subsection{Results}

\subsubsection{Samples}

Five seafloor lava samples were selected out of several dozen samples collected from the EPR as 'representative types' for the analyses presented here. These represent a variety of flow morphologies, ages (up to 20 kyrs based on the crustal spreading rate and distance to the ridge axis), and extent of alteration. Table 3.1 describes the location, site, and characteristics for each basaltic rock sample and one deep seawater sample. The 
youngest lava samples were collected from within the axial summit trough (AST), an elongate volcanic collapse feature at the spreading axis where lava is erupted and hydrothermal venting is focused (Fornari et al., 1998). The majority of samples were collected from the ridge flanks out to $\sim 3 \mathrm{~km}$ from the AST and represent a variety of lava flows that differ in age and morphology. All lavas have a normal mid-ocean ridge basaltic (N-MORB) composition (Sims et al., 2002) and are commonly encrusted in a rind (mm- to $\mathrm{cm}$ - thick) of basaltic glass, a result of the rapid cooling of lava in cold seawater. These basalts are generally fresh, as characterized by the abundance of unaltered glass. The representative "fresh glass" sample collected from within the AST, sample FG (Table 3.1), is a glass nodule that appears to be the youngest (near zero-age) based on location and "freshness", or lack of visible alteration products. In the majority of samples, however, the glassy portions directly exposed to seawater on the surfaces and in fractures have been replaced by palagonite (a mixture of amorphous, hydrated glass) and poorly crystallized secondary minerals such as clays and Fe-oxyhydroxides (Stroncik and Schmincke, 2001). Mineralogical analyses of the secondary mineral assemblages using microscopic and spectroscopic techniques will be presented in a forthcoming communication. Three of the analyzed samples were classified as "slightly altered", for instance samples $\mathrm{FeO} 1$ and $\mathrm{FeO} 2$ (lavas with thin Fe-oxide crusts) and sample $\mathrm{AlO}$ (lava coated in a thin layer of primarily aluminum hydroxide). Older lavas exhibit more extensive glass alteration, such as clay and Fe-oxide filled veins and mm-thick palagonite rims. Some of the samples are encrusted in mm-thick Fe and Mn-oxide minerals. The 
oldest, most altered lava with a ferromanganense oxide crust used in this study is sample $\mathrm{MnO}$.

\subsubsection{Phylogenetic diversity}

Clones from the five different seafloor lavas and deep seawater sample represent a total of 17 distinct bacterial taxonomic groups, and the results showing the relative proportions of the different groups in each clone library (sample) are summarized in Fig. 3.2. Clones from all five subdivisions of Proteobacteria $(\alpha, \beta, \gamma, \delta$, and $\varepsilon)$ have been recovered and account for the majority of sequences in each clone library, but not all subdivisions are present in every sample. With the exception of the aluminum oxide coated sample (AlO), sequences belonging to $\gamma$-Proteobacteria dominate all clone libraries and account for $22-37 \%$ of each basaltic library and $89 \%$ of the DSW library. The $\gamma$-Proteobacteria clones for each sample are quite diverse, distributed amongst at least 15 different families (Fig. 3.3). Many of the clones cluster within groups that are metabolically diverse and ubiquitous in marine environments, such as Alteromonadaceae, Halomonadaceae, and Pseudomonadaceae. Clones belonging to families that are more physiologically constrained include Methylococcaceae, methane oxidizing bacteria, and Ectothiorhodospiraceae, bacteria that predominately use sulfide as an electron donor. Species most closely aligning with Colwelliaceae, predominantly psychrophilic bacteria, and Idiomarinaceae, aerobic chemoorganotrophs primarily isolated from deep-sea environments, were also recovered in the clone libraries. The majority of the sequences isolated from seafloor lavas are most closely related to other clones from a wide variety 
of environments (see Appendix 3.1), including Japan Trench cold seep sediments (Li et al., 1999), gas-hydrate associated sediments (Knittel et al., 2003), and deep continental crust aquifer (Lin et al., 2006a) based on BLAST analyses (Altschul et al., 1997). As seen in the phylogenetic tree (Fig. 3.3), many of these uncultured species (including those from this study) form their own monophyletic group and are only distantly related to any cultured microorganisms.

$\alpha$-Proteobacteria sequences dominate $\mathrm{AlO}$, the $\mathrm{Al}$ hydroxide encrusted lava clone library (46\%), and comprise $8-30 \%$ of the others (Fig. 3.2). Many of the $\alpha-$ Proteobacteria clones (Fig. 3.4) are related to members of the family Sphingomonadaceae, physiologically and ecologically diverse bacteria that are predominantly aerobic chemoheterotrophs, as well as the family Hyphomicrobiaceae, a group characterized by hyphal, prosthecate and budding microorganisms with either chemoorganotrophic (e.g. methane or manganese oxidation) or autotrophic capabilities that are often found in oligotrophic environments. A number of the $\alpha$-Proteobacteria clones from one of the Fe oxide coated samples $(\mathrm{FeO} 2)$, the ferromanganese enrusted sample $(\mathrm{MnO})$, and sample AlO are most similar (Fig. 3.4 and Appendix 3.1) to various uncultured clones obtained from basaltic lavas near the Juan de Fuca mid-ocean ridge (GenBank acc. DQ070827 and DQ070833).

$\beta$-Proteobacteria are most abundant (Fig. 3.2) in the freshest sample, FG, representing $16 \%$ of that clone library. $\beta$-Proteobacteria clones were obtained from only 3 libraries (FG, FeO1, and deep seawater). Many of these sequences cluster with the family Comamonadaceae (Fig. 3.4), which are a physiologically diverse group of 
bacteria able to grow heterotrophically or chemolithotrophically such as through hydrogen oxidation. Clones representing the $\delta$-Proteobacteria were recovered from all seafloor lava samples, but not the deep seawater sample (Fig 3.2). The highest proportion (16\%) of $\delta$-Proteobacteria are observed in one of the Fe-oxide encrusted samples (FeO1). The $\delta$-Proteobacteria contain a number of species that are known for their dissimilatory sulfate and sulfur reducing capabilities, such as Desulfovibrio, however most sequences from this study do not cluster with these organisms (Fig. 3.4) or any cultured bacteria. Several clones from libraries FG were most similar (Appendix 3.1) to clones obtained from warm ridge-flank fluids emanating from basaltic ocean crust (Huber et al., 2006), and several other from this library and $\mathrm{FeO} 1$ are most closely related to the basaltic glass clones from the Juan de Fuca ridge mentioned earlier (GenBank acc. DQ070830). Sequences belonging to $\varepsilon$-Proteobacteria were recovered only from sample FG and make up $18 \%$ of the total library. Unlike any other phylogenetic groups, all clones that are members of the $\varepsilon$-Proteobacteria class (13 total) are dominated by a single phylotype, which is $99 \%$ similar to another clone isolated from basaltic glass found at the EPR in a separate study (GenBank acc. DQ070790).

In addition to Proteobacteria, clones belonging to a total of 11 other bacterial groups were recovered from the clone libraries. Planctomycetes and Actinobacteria sequences have been recovered from all basalt samples, ranging from 1-12\% and 6-11\% respectively (Fig. 3.2). Planctomycetes are budding bacteria that are ubiquitous in the environment but poorly understood with regard to physiology; however, species such as P. bekefii have been observed to accumulate Mn and Fe oxide particles (Schmidt et al., 
1982), suggesting the formation of these minerals could be microbially catalyzed. Most Planctomycetes clones from this study (Fig. 3.5), not surprisingly, are more closely related to clones from other deep marine environments, for example from warm basement fluids from the eastern Juan de Fuca ridge flank (Huber et al., 2006) and young basaltic lavas (GenBank acc. DQ070834) as well as sediments from the Antarctic continental shelf (Bowman and McCuaig, 2003). Although a few Actinobacteria clones are closely related (97-99\%) to cultured species of Micrococcus and Nesterenkonia, many of the clones form their own monophyletic groups with other uncultured microorganisms. This includes clones obtained from ridge flank fluids (Huber et al., 2006), an inactive deep-sea hydrothermal chimney (Suzuki et al., 2004), and hydrothermal sediment (Lopez-Garcia et al., 2003). The CFB group (Cytophaga-Flavobacteria-Bacteroides), although not represented in the fresh glass sample (FG), accounts for $2-11 \%$ of the clone libraries for the "slightly altered" samples. The CFB group includes mostly Flexibacteraceae, unicellular gliding bacteria with varying physiologies, and Flavobacteriaceae, heterotrophic bacteria that cover widely diverse ecological niches and physiologies.

A small proportion $(0-6 \%)$ of the clones belongs to one or more of the following phyla (Fig. 3.2): Verrucomicrobia, Firmicutes, Acidobacteria, Nitrospirae, Gemmatimonadetes, Spirochaetes, Chlymdiaea, Candidatus Scalindua brodae, and Candidate Division OP11. It is difficult to place most of these clones in any specific rank past the phylum level with any confidence because they are related primarily to other unclassified environmental clones. Two exceptions are the Nitrospirae clones (Fig. 3.5) in which sequences are $95 \%$ similar to Nitrospira marina (Teske et al., 1994), a 
chemolithoautotrophic nitrite oxidizer, and Candidatus Scalindua brodae (Schmid et al., 2003), an anaerobic ammonium oxidizing (ANAMMOX) bacterium. A significant number of clones, $2-13 \%$, from each library obtained from lava samples could not be assigned a phyla (Unclassified; Fig. 3.2) because they have confidence thresholds lower than $80 \%$ as assigned by the Ribosomal Database Project, RDP (Cole et al., 2003).

\subsubsection{Quantification of microbial communities}

Real time, quantitative PCR is used to estimate the domain level abundance of epi- and endolithic prokaryotic communities inhabiting each sample by targeting the $16 \mathrm{~S}$ rRNA gene for bacteria and archaea. Gene copy numbers and estimated cell biomass are summarized in Table 3.2. Bacterial gene copies range from $\sim 10^{7}$ to $5 \times 10^{9}$ copies per gram crushed basalt, which is equivalent to approximately $3 \times 10^{6}$ to $10^{9}$ cells $^{-1}$ rock. The lowest DNA concentration, $1 \times 10^{7}$ copies $\mathrm{g}^{-1}$, was detected in the fresh, glassy lava sample (FG). DNA concentrations for samples showing slight alteration, the Al hydroxide (AlO) and Fe oxide (FeO1) encrusted samples, show an approximately 10-fold increase. The ferromanganese oxide encrusted sample (MnO), showing the most pervasive alteration, contains the highest concentration of DNA by over 2 orders of magnitude compared to sample FG. In all analyzed seafloor basalts, the gene copy numbers for the bacterial domain are 1 to 2 orders of magnitude higher than those for the archaeal domain. Therefore, bacteria dominate the prokaryotic population accounting for $88-96 \%$ of the total. These results are confirmed by an independent qPCR assay (data not shown) using universal qPCR primer sets targeting the $16 \mathrm{~S}$ gene for the total prokaryotic 
population, which show prokaryote gene copies approximately equal (on the same order of magnitude) to that measured for bacteria. The DNA concentrations for sample FeO1 and DSW could not be determined due to insufficient quantities of DNA extract.

\subsubsection{Rarefaction analysis and richness estimates}

The relative species richness of bacteria observed within each sample is determined through rarefaction analysis. For rarefaction, the number of observed operational taxonomic units (OTUs) assigned by DOTUR (Schloss and Handelsman, 2005) is compared to the sequencing effort for each clone library. In this study, OTUs are defined by a 0.03 distance level $\left(\mathrm{OTU}_{0.03}\right)$, so sequences with $\geq 97 \%$ similarity are designated to a single OTU. This taxonomic distinction is often used to indicate species level variation (Ley et al., 2006; Schloss and Handelsman, 2005). Rarefaction curves (Fig. 3.3) show that the greatest richness (number of OTUs), based on the slope of each line, is observed in the slightly altered, Fe oxide coated sample FeO1. Approximately 46 different OTUs are observed from only 52 sequenced clones. The other Fe hydroxide coated sample, $\mathrm{FeO} 2$, and the oldest, most altered ferromanganese encrusted sample, $\mathrm{MnO}$, exhibit indistinguishable rarefaction curves, in which approximately 38 phylotypes are detected from 52 sequences. More clones have been successfully obtained for these two samples, which show an OTU/total clones ratio of 54/81 for $\mathrm{FeO} 2$ and 55/84 for MnO. Based on overlapping standard errors ( $95 \%$ confidence intervals not shown), the rarefaction curves for the slightly altered, Al hydroxide coated sample (AlO) and the fresh glass sample (FG) also can not be differentiated. These two basalt samples reveal 
the lowest relative species richness with $\sim 34 \pm 4$ and $30 \pm 4$ OTUs, respectively, at the comparative sequencing effort of 52 clones. All of the seafloor lava samples have steep, near linear trends, indicating that more OTUs would be obtained with a greater sequencing effort. The deep seawater sample (DSW), however, reaches a near asymptotic value of 11 clones.

A similar pattern of relative richness is estimated for the different samples using nonparametric richness estimators, which consider unsampled organisms (Table 3.3). The standard error (95\% CI), however, is large for these samples. Chao1 (Chao, 1984) richness estimates for $\mathrm{AlO}$ and $\mathrm{FG}$, the samples with the lowest observed diversity, predict 74 and 84 different OTUs respectively for a fully sampled community. Samples $\mathrm{FeO} 2$ and $\mathrm{MnO}$ have Chao1 estimates of 145 and 153, whereas sample FeO1 has 157 predicted phylotypes. It is noted that rarefaction curves of the Chaol estimates (not shown) do not reach a clear asymptotic value for any of the basalt samples, similar to the rarefaction curves of observed richness, and should be regarded as minimum estimates.

\subsubsection{Bacterial community overlap and distribution estimates}

The similarities in the OTUs detected and their abundance and distribution in each sample are compared using a variety of statistical analyses implemented in the program SONS (Schloss and Handelsman, 2006) that are based on estimated species richness (Chao1). The number of shared OTUs $\left(\mathrm{OTU}_{0.03}\right)$ between each sample, calculated by the shared Chaol richness estimator (Chao et al., 2006), is shown in Table 3.3. The results show that samples FeO1 and FeO2 (the two Fe-oxide coated samples) share the greatest 
number of OTUs (42) compared to any other two samples. Sample FeO2 also shares a large number of OTUs (40) with sample $\mathrm{MnO}$ (a Mn and Fe-oxide encrusted sample). Shared richness is observed in all of the basalt samples, with the lowest number of shared OTUs generally found relative to the fresh glass sample (FG) with the exception of sample FeO1, which shares approximately 23 types. In contrast, the deep seawater sample (DSW) only shared richness with two samples, FG and FeO1, of which the majority (7) was shared with FG.

The overlap in bacterial community membership between two samples, based on the fraction of sequences that belong to shared OTUs, is estimated by the abundancebased Jaccard $\left(\mathrm{J}_{\text {abund }}\right)$ similarity index (Chao et al., 2005) as shown in Table 3.4 for an OTU definition of 0.03 . The overlap measurements represent the probability that an OTU recovered in one community will also be recovered in the other community, so an overlap index of 1.00 means there is a $100 \%$ chance that a randomly chosen OTU will be represented in both communities. The trends in bacterial community overlap between the several basalt-hosted and deep seawater samples are similar to what is observed for the shared richness estimates, however the $\mathrm{J}_{\text {abund }}$ calculated for samples $\mathrm{MnO}$ and $\mathrm{FeO} 2$ (0.27) is slightly greater than between $\mathrm{FeO} 1$ and $\mathrm{FeO} 2(0.24)$. The overlap between $\mathrm{AlO}$, and $\mathrm{MnO}$ and $\mathrm{FeO} 2$ is similarly high (0.20 and 0.19 respectively) but is, however, surprisingly low compared with the other Fe-oxide sample (0.05). The $\mathrm{J}_{\text {abund }}$ calculated for fresh glass and deep seawater is 0.17 .

Community overlap differs from community structure in that overlap considers only membership (OTUs) in each sample, whereas structure accounts for similarities in 
both membership and abundance. Estimates of community structure similarity $\left(\theta_{\mathrm{YC}}\right)$ shown in Table 3.4 were determined using the nonparametric maximum likelihood estimator by Yue and Clayton (2005) at an $\mathrm{OTU}_{0.03}$ definition. The similarity $\left(\theta_{\mathrm{YC}}\right)$ measured for $\mathrm{FeO} 1$ and $\mathrm{FeO} 2$ is $0.65(\mathrm{SE}=0.04)$. In comparison, the next highest value is $0.19(\mathrm{SE}=0.06)$ for samples $\mathrm{MnO}$ and $\mathrm{FeO}$, and in general the values comparing the basalt samples range from 0.02 to 0.19 with the above exception. The basalt samples FG and $\mathrm{FeO} 1$ have similar $\theta_{\mathrm{YC}}$ values of 0.07 when compared to the deep seawater sample.

\subsection{Discussion}

Two other studies have examined the phylogenetic diversity of young crust at the ocean floor (Lysnes et al., 2004a; Thorseth et al., 2001); however, the bacterial diversity observed in EPR basalts from this study is substantially larger. Previous studies obtained sequences belonging to 3 (Thorseth et al., 2001) and 8 (Lysnes et al., 2004a) major taxonomic groups, whereas this study recovered sequences belonging to 17 different groups of the same rank, as seen in Fig. 3.2. This discrepancy could be a result of either location (previous studies examined basalts from the Arctic spreading ridges) or differences in methodology (short fragment 16S rRNA sequences obtained by denaturing gradient gel electrophoresis fingerprinting versus full-length $16 \mathrm{~S}$ rRNA clone library construction). In both this study and the Lysnes et al (2004) study, most of the recovered sequences are from a diverse population of $\gamma$-Proteobacteria, only a few of which are closely related to sequences recovered from deep seawater, despite the dominance of this group in the DSW clone library. It is important to note here that although we discuss the 
proportions of clones belonging to specific taxonomic groups throughout the study (e.g. $\gamma$ -Proteobacteria comprise $89 \%$ of the analyzed DSW clone library), we do not suggest that these ratios correspond to the abundances of these microorganisms in the ocean crust habitat - cellular abundances need to be determined by alternative analyses such as qPCR or fluorescent in situ hybridization, which were only performed at the domain level in this study. However, sequences in this study were obtained from each sample using the same methodology (from DNA extraction through sequencing) for each sample and PCR primer sets do not appear to favor a certain taxonomic rank, so technique biases are of little concern in comparing the relative proportions of each clone library in this study. Interestingly, the number of observed sequences within a specific group typically correlates with the number of different OTUs within that group because the species richness in this study is so high (Fig 3.6). This relationship holds true for all groups except the $\varepsilon$-Proteobacteria, for which a single OTU represents 13 clones.

Because $\gamma$-Proteobacteria sequences are most prevalent in many of the clone libraries recovered from EPR basalts and because they do not closely match those found in ambient bottom seawater, we hypothesize that many of these microorganisms are indigenous to the basalt habitat - bacteria that may be obtaining energy from rock alteration processes. This observation also holds true for the group with the second highest relative proportion of clones, the $\alpha$-Proteobacteria. Indeed, the dichotomy in bacterial diversity observed between the EPR basalts and surrounding seawater is rather large for all groups. Furthermore, many of the sequences are most similar to uncultured environmental clones and isolates obtained from other seafloor lavas (Appendix 3.1), 
further suggesting that much of the biotope is specific to this bare-rock environment. A number of closest BLAST matches to the EPR basalts are sequences belonging to a number of different groups ( $\alpha$ - and $\delta$-Proteobacteria and Planctomycetes) obtained from Juan de Fuca mid-ocean ridge basalts (GenBank pop. set DQ070815-DQ070835) as well as $\varepsilon$-Proteobacteria and Planctomycetes obtained from EPR basalts in an independent study (GenBank pop. set DQ070789-DQ070814). Several sequences belonging to $\delta$-Proteobacteria, Planctomycetes, Actinobacteria, and Acidobacteria are most similar to uncultured bacteria found in warm fluids emanating from young basaltic crust at the eastern Juan de Fuca Ridge flank (Huber et al., 2006).

If the basalt-hosted biotope is specific to this habitat and underpinned by chemolithoautotrophs, it is expected that some of the recovered phylotypes exhibit aerobic lithoautotrophic metabolisms linked to chemical redox reactions occurring as a direct result of basalt oxidation and hydration reactions or from the input of hydrothermal fluids, for example sulfide, iron, manganese, hydrogen, and methane oxidation (Edwards et al., 2005). Redox gradients developing on basalt surfaces may also be influenced by the formation of biofilms and secondary mineral precipitates (from hydrothermal input or as a result of basalt oxidation) encrusting the lavas. These processes may create niches capable of supporting chemosynthetic microorganisms with anaerobic metabolic pathways (e.g., sulfate reduction, methanogenesis, anaerobic ammonia oxidation, or anaerobic iron oxidation). We note here that the physiologies of uncultured clones inferred through phylogenetic relationships are only speculative at this point. Nevertheless, some genetically constrained groups of microorganisms, such as the sulfur- 
oxidizing bacteria that belong to the family Ectothiorhodospiraceae, exhibit more defined metabolic capabilities; and many of the phylogenetically related microorganisms demonstrate metabolisms that are bioenergetically favorable in this environmentallowing for more confident inferences of potential physiologies. For example, one of the $\mathrm{MnO}$ clones is $92 \%$ similar to Thioalkalivibrio thiocyanodenitrificans (GenBank acc. AY360060), a sulfur oxidizing, dentrifying $\gamma$-Proteobacteria (Sorokin et al., 2004) that is a member of the Ectothiorhodospiraceae family which generally uses sulfide as an electron donor and produces external sulfur globules. The presence of several other closely related species also suggest that microbially catalyzed sulfur oxidation is occurring on the basalt surfaces, which could initially be colonized by hydrothermal vent bacteria. A sulfur-oxidizing bacterium isolated from a hydrothermal vent, species NDII1.1 (GenBank acc. AF170424), was related (92\% similar) to a clone in the fresh glass sample. Additionally, the single phylotype recovered within the $\varepsilon$-Proteobacteria clusters near Helicobacteraceae and is $95 \%$ similar to a chemolithoautrophic hydrogenand sulfur-oxidizing bacterium isolated from a hydrothermal vent polychaete nest (Takai et al., 2006). Previous studies have demonstrated that a number of chemolithoautotrophic sulfur-oxidizing bacteria from deep-sea hydrothermal vents can use a variety of metal sulfide minerals for metabolic growth (Eberhard et al., 1995; Wirsen et al., 1993). In addition to sulfur cycling, many related species suggest that some microbes inhabiting the ocean crust obtain energy for metabolic growth through the oxidation of methane and intermediate 1-C compound species. For instance, several $\gamma$-Proteobacteria sequences in sample $\mathrm{FeO} 2$ were $95 \%$ similar to a methanotrophic bacterium Methylobacter luteus 
(Gulledge et al., 2001), and an $\alpha$-Proteobacteria sequence recovered from sample FG is 99\% similar to the methylotroph Methylobacterium fujisawaens (GenBank acc. AJ250801).

Previously, some of the other authors (Bach and Edwards, 2003; Edwards et al., 2005) demonstrated that a significant amount of metabolic energy can be gained by chemolithoautotrophs via aerobic Fe (II) oxidation (approximately $65 \mathrm{~kJ} \mathrm{~mol}^{-1}$ ) reaction on basalt glass. Furthermore, laboratory experiments with Fe-oxidizing bacteria obtained from the seafloor (Edwards et al., 2004; Edwards et al., 2003) demonstrate that these microorganisms greatly enhance the dissolution rate of basalt glass under seafloor conditions. Many of the seafloor lavas collected for this study are encrusted in a thin layer of Fe hydroxide (samples $\mathrm{FeO} 1$ and $\mathrm{FeO} 2$ ), which we hypothesize result from lowtemperature alteration processes potentially catalyzed by microbial activity. Although sample $\mathrm{MnO}$ is encrusted in a thick layer of Fe and $\mathrm{Mn}$ oxides, it is likely that these precipitates did not result from rock weathering but are the result of distant hydrothermal input and subsequent oxidation in the water column. None of the sequences obtained from any of the EPR basalts, however, could be clearly regarded as potential Feoxidizers. This is not entirely surprising: Bacteria capable of Fe-oxidation do not form tightly constrained families or classes based on 16S rRNA phylogenetic analysis, unlike many microorganisms with metabolisms based on sulfur or nitrogen redox cycling. Rather, many of the cultured organisms that exhibit Fe-oxidation are phylogenetically related to groups that utilize a wide variety of substrates for metabolic growth. For instance, the neutrophilic Fe-oxidizing species used for glass dissolution experiments 
cluster most closely with Hyphomicrobiaceae (Edwards et al., 2003), a group that includes denitrifiers, nitrogen fixers, methylotrophs, and chemoorganotrophs. Many strains are also capable of more than one type of metabolism (e.g. Sulfobacillus acidophilus can oxidize inorganic sources of Fe or S for growth (Norris et al., 1996)).

If chemolithoautotrophic bacteria supported by basalt alteration reactions compose the trophic base for ocean crust communities, then phylogenetic analyses should also convincingly identify heterotrophic microorganisms. Indeed, a number of sequences recovered from the basalt clone libraries indicate that microbes likely capable of mixotrophic or chemoorganotrophic metabolisms are also present in seafloor lavas. An Actinobacterial clone from the fresh glass sample is $99 \%$ similar to a heterotrophic Micrococcaceae sp. isolated from young Hawaiian volcanics (DQ490457), and another is 98\% similar to Nesterenkonia abyssinica, a heterotrophic, moderate halophile isolated from an Ethiopian soda lake (Delgado et al., 2006). Another clone from sample FG is most similar to a polycyclic aromatic hydrocarbon degrading bacterium, Kordiimonas gwangyangensis (Kwon et al., 2005). Additionally, many clones belong to families that are generally demonstrated to have heterotrophic metabolisms, such as Idiomarinaceae ( $\gamma$ -Proteobacteria) and Flavobacteriaceae (CFB group). Although dissolved organic carbon concentration in the deep-sea is present $(\sim 40 \mu \mathrm{M})$, it has been demonstrated that most of this carbon pool is refractory to microbial oxidation (Barber, 1968). These observations provide further support that chemolithoautotrophic primary biomass production is fueling the ocean crust biotope. 
In addition to microbially influenced $\mathrm{Fe}, \mathrm{S}$, and $\mathrm{CH}_{4}$ redox cycling occurring in the ocean crust, it is suggested that nitrogen cycling bacteria, such as nitrate reducers (Bach and Edwards, 2003; Cowen et al., 2003), could also exist in this environment. Phylogenetic analyses of the EPR basalts imply that several different nitrogen redox reactions are occurring in bacteria inhabiting these seafloor lavas. For example, a $\beta-$ Proteobacteria clone recovered from the fresh glass sample is $97 \%$ similar to a lithotrophic Nitrosospira sp. (Purkhold et al., 2003) that gains energy through ammoniaoxidation. Another clone from that sample is $98 \%$ similar to a denitrifying Acidovorax sp. isolated from activated sludge (Khan et al., 2002). The Candidatus Scalindua brodae clone recovered from one of the Fe-oxide encrusted samples is $97 \%$ similar to a recently identified anaerobic ammonia oxidizing (ANAMMOX) bacterium (Schmid et al., 2003). Additionally, a couple of clones from $\mathrm{FeO} 2$ and the ferromanganese oxide encrusted sample belong to the phylum Nitrospira, including one that is $95 \%$ similar to Nitrospira marina, a chemolithoautotrophic nitrite oxidizer (Teske et al., 1994). Indirect evidence for nitrate reduction is provided by the clones that cluster with Pseudomonas, of which a number of these species are nitrate reducers. Interestingly, a number of nitrate reducing bacteria also have Fe oxidizing capabilities (Straub et al., 2004). The extent to which nitrogen cycling is directly linked to the ocean crust habitat is not entirely understood as only trace amounts of dissolved $\mathrm{N}$ exist in basalts. Regardless, the results presented here suggest that the metabolic capabilities of bacteria in this habitat could potentially play an important role in the biogeochemical cycling of this element. 
The extent to which microbial communities inhabiting the ocean crust influence the geochemical cycles of elements like $\mathrm{S}, \mathrm{Fe}, \mathrm{Mn}, \mathrm{C}$, and $\mathrm{N}$ is dependent on a number of factors other than the metabolic capabilities of the microbes (e.g. lateral and vertical extent of biosphere, cell density, and microbial activity). We previously demonstrated (Chapter 2) through catalyzed reporter deposition-fluorescent in situ hybridization (CARD-FISH) analyses that metabolically active prokaryotic communities, heavily dominated by Bacteria, abundantly colonize seafloor basalts. Compared to seawater cell densities of $\sim 10^{4}$ cells $\mathrm{mL}^{-1}$ (Karner et al., 2001), qPCR measurements show that the prokaryotic biomass in seafloor lavas is significantly more dense, $\sim 3 \times 10^{6}$ to $1 \times 10^{9}$ cells $\mathrm{g}^{-1}$ rock. These cell densities are consistent with the predicted biomass, assuming chemolithoautotrophic microorganisms are catalyzing Fe and S oxidation reactions in the basalts (Chapter 2, Bach and Edwards, 2003), serving as the trophic base for this microbial ecosystem. Relative to the youngest, freshest sample (FG), the 10 -fold to 1000-fold increase in bacterial biomass observed in the slightly altered samples (A1O and FeO2) and the oldest, most altered sample $(\mathrm{MnO})$, respectively, demonstrates that there is a direct correlation with microbial abundance and basalt alteration. Although the spatial extent of the ocean crust biosphere is not yet elucidated, results of our qPCR and CARDFISH analyses suggest that the potential for bacteria to impact the geochemical evolution of the ocean crust and deep seawater near the MOR system is substantial.

Although the bacterial biomass abundance clearly correlates with the increase in age and alteration state of the rock, the species richness (Figs. 3.6 and Table 3.2, respectively) and community structure analyses for each lava sample, though broadly 
correlating positively with alteration, portrays more complicated relationships. As expected, the least species richness determined using rarefaction analysis is in the young, fresh glass sample (FG). The number of observed OTUs, however, is essentially indistinguishable from the slightly altered, Al hydroxide coated sample (AlO), despite the order of magnitude increase in cell density between the two. It is unlikely that microorganisms are actively participating in the formation of the $\mathrm{Al}$ hydroxide crust as $\mathrm{Al}$ is not a redox reactive element in Earth surface environments. The presence of the $\mathrm{Al}$ oxides is likely due to abiotic, low-temperature hydrothermal precipitation. This nonredox active crust could passivate some of the basaltic glass surface with respect to microbial activity. Therefore, it is not surprising that the species diversity in sample AlO is not as high as that found in the other altered samples (FeO1, FeO2, and $\mathrm{Mn}$ ). This interpretation, however, does not adequately explain the increase in biomass. Interestingly, all three community distribution analyses $\left(\mathrm{S}_{\mathrm{Chao} 1}, \mathrm{~J}_{\mathrm{abund}}\right.$, and $\left.\theta_{\mathrm{YC}}\right)$ suggest that there is very little overlap or similarity in the membership and structure between samples $\mathrm{AlO}$ and $\mathrm{FG}$, but the microbial communities in $\mathrm{AlO}$ are more like those found in the other slightly altered samples (FeO1 and FeO2). So although fewer OTUs were obtained from the Al hydroxide coated sample relative to the other oxide coated samples, the types and abundances of OTUs recovered from these lavas imply that the microbial community is evolving to match the availability and variety of electron donors and acceptors present on the basalt surfaces.

The correlation between basalt alteration state and species richness also does not hold for the oldest, most altered sample $(\mathrm{MnO})$, even though the bacterial density is 100 - 
1000 times greater than in any other basalt sample analyzed in this study. Both rarefaction analysis and the Chaol estimator show that the slightly altered, Fe oxide encrusted sample $\mathrm{FeO} 1$ harbors the greatest number of types of bacteria. Because rarefaction curves failed to reach an asymptote for all basalt clone libraries, indicating that the microbial community has not been fully sampled, it is possible that the true relative diversity may change with greater sequencing. The observed richness, however, does not explain differences in community complexity with respect to each sample, therefore comparisons of community overlap and structure may be more beneficial to discern the relationship with basalt geochemistry. For instance, the composition (structure and membership) of the bacterial communities recovered from sample $\mathrm{FeO} 2$ is most similar to both the other Fe oxide coated sample and the ferromanganese encrusted sample. This may suggest that the bacteria inhabiting these samples are in some way controlled by the geochemical reactions occurring on the basalts, furthermore contributing to the formation of these oxide crusts. This interpretation is somewhat complicated by the observation that the community similarity measurements are low between samples FeO1 and $\mathrm{MnO}$. This, however, could largely be due to the undersampling of the clone libraries, particularly FeO1.

This study clearly exposes a positive correlation between basalt alteration state and both the abundance and structure of the bacterial communities existing in this barerock environment. The foundation for this relationship, however, cannot be established with the data presented here. In other words, we cannot definitely distinguish through these analyses if the microorganisms are directly contributing to the basalt alteration 
processes or if the microbial communities are controlled by redox gradients formed during abiotic basalt oxidation/hydration reactions, hydrothermal input, and/or secondary mineral precipitation. Although difficult to resolve, we suspect that both factors contribute to the phylogenetic and physiological diversity observed in the seafloor lavas from the EPR. Further studies, and larger data sets, are needed to resolve these possibilities. Nonetheless, it is becoming increasingly apparent that the ocean crust biosphere is extensive and may significantly affect the geochemical exchange between crust and seawater.

\subsection{Experimental Procedures}

\subsubsection{Study site and sample collection}

Seafloor lavas were sampled for this study from the East Pacific Rise (EPR) between $9^{\circ} 28^{\prime} \mathrm{N}$ and $9^{\circ} 50^{\prime} \mathrm{N}$ (Fig. 3.1). Exposed basalt samples were collected during cruises AT11-7 and AT11-20 on the R/V Atlantis in February and November, 2004, respectively. The DSV Alvin was used to sample rocks samples from in-situ volcanic flows at the seafloor. Rock samples were placed in bio-boxes containing sterile freshwater, allowed to fill with ambient bottom seawater, sealed to minimize contamination from the upper water column, and brought to the surface. Deep seawater was collected using Niskin bottles that were fired at the seafloor.

\subsubsection{Clone libraries and phylogenetic analyses}


Environmental DNA from seafloor lavas and deep seawater was extracted, amplified, cloned, and sequenced using the protocols and methods outlined in Chapter 2, All sequences used in this study will be submitted to the GenBank Database pending publication of Chapter 2. Sequence data were submitted to the online BLAST (Basic Local Alignment Search Tool) (Altschul et al., 1997) program to be compared against the GenBank 16S rRNA database for identifying closely related environmental clones and cultured isolates. The phylogenetic affiliation of each clone was assigned using an $80 \%$ confidence threshold by the taxonomical "Classifier" program available through the Ribosomal Database Project, RDP (Cole et al., 2003).

Aligned sequences from the clone libraries, along with closely related environmental clones and cultures, were selected for phylogenetic tree construction. Tree topology and branch lengths were determined using GARLI (www.bio.utexas.edu/ faculty/antisense/garli/ Garli.html; (Zwickl, 2006)), which uses maximum likelihood criterion for distance analysis. MODELTEST (Posada and Crandall, 1998) confirmed the use of a General Time Reversible (GTR) model with gamma rate heterogeneity and an estimated proportion of invariable sites. Maximum likelihood bootstrapping was carried out with 1,000 replicates for the non-proteobacterial tree, and 500 replicates for the other trees. Bootstrap proportions were obtained by reading all trees into PAUP* to obtain a majority rule consensus. Phylogenetic trees were visualized with TREEVIEW (Page, 1996).

\subsubsection{Quantitative PCR (qPCR) analysis}


qPCR analyses to estimate bacterial and archaeal biomass was carried out on subaliquots of environmental DNA extracted for phylogenetic analyses. Previously designed domain level primer and probe sets were used for quantification of bacterial (Nadkarni et al., 2002) and archaeal (Takai and Horikoshi, 2000) 16S rRNA genes. Protocols for amplification and quantification are outlined in Chapter 2. A conversion factor of 4.1 (16S rDNA gene copies cell ${ }^{-1}$ ) was used for determine bacterial and total prokaryote cell abundances, whereas 1.6 was used to convert archaeal gene copies to cell numbers (Klappenbach et al., 2001). Biomass (\% archaea or bacteria) was quantified by summing bacterial and archaeal cell abundances to obtain total cell numbers.

\subsubsection{Rarefaction analysis and Diversity comparisons}

The computer program DOTUR (http://www.plantpath.wisc.edu/fac/joh/ DOTUR.html; (Schloss and Handelsman, 2005)) was used to describe the diversity of microbial communities inhabiting variably altered basaltic lavas. A distance matrix generated in ARB from the aligned sequence data for each sample was used as the input. The program assigned sequences to operational taxonomic units (OTUs) using the furthest-neighbor algorithm, which were then used to construct rarefaction curves and calculate species richness estimators such as the bias-corrected Chaol richness estimator (Chao, 1984) along with $95 \%$ confidence intervals. A distance level of 0.03 was selected to compare sequences for both rarefaction and richness indicators.

The newly created computer program SONS (Schloss and Handelsman, 2006); http://www.plantpath.wisc.edu/fac/joh/sons.html) was used to compare the bacterial 
community structure and OTU overlap amongst the different seafloor lavas. This program was selected because the analyses are performed at discrete phylogenetic levels. Specifically for this study, we compared the community structure at an OTU definition of 0.03 , as was used in the rarefaction analyses and richness estimators. The program used the "list" output file from the previous DOTUR analyses to calculate the similarity $\left(\theta_{\mathrm{YC}}\right)$ of the community structures (Yue and Clayton, 2005), the abundance-based Jaccard

( $\left.\mathrm{J}_{\text {abund }}\right)$ similarity index (Chao et al., 2005), and the shared Chaol $\left(\mathrm{S}_{1,2}\right.$ Chao $)$ richness estimates (Chao et al., 2006).

\subsection{Acknowledgements}

The authors thank Dan Fornari, Maurice Tivey, and Hans Schouten (WHOI) for accommodating C.M.S. and W.B. on research cruise AT11-7. Everyone at the Josephine Bay Paul Center at the Marine Biological Laboratories, especially Hilary Morrison and David Mark Welch, have been tremendously helpful and resourceful with sequencing and phylogenetic analyses. Eric Webb and Stefan Sievert (WHOI) shared their lab space and equipment for sequencing. Patrick Schloss (U. Wisconsin-Madison/U. MassachusettsAmherst) has been of great assistance regarding DOTUR and SONS analyses. This research was supported by RIDGE 2000 Grant \#OCE-0241791awarded to K.J.E. and W.B. and NASA Astrobiology Institute Award NNA04CC04A to KJE. 


\subsection{Tables}

Table 3.1. Description of seafloor basalts sampled from the EPR.

\begin{tabular}{|c|c|c|c|c|c|}
\hline Sample $^{\mathrm{a}}$ & $\begin{array}{l}\text { Latitude } \\
\left({ }^{\circ} \mathrm{N}\right)\end{array}$ & $\begin{array}{l}\text { Longtitude } \\
\left({ }^{\circ} \mathrm{W}\right)\end{array}$ & $\begin{array}{l}\text { Depth } \\
\text { (m) }\end{array}$ & Field site description & Sample description \\
\hline $\begin{array}{l}\text { FG } \\
\text { (EPR3967-O2) }\end{array}$ & $9^{\circ} 49^{\prime} 42^{\prime \prime}$ & $104^{\circ} 16^{\prime} 32^{\prime \prime}$ & 2539 & $\begin{array}{l}\text { Pillow mound from within } \\
\text { the AST \& away from } \\
\text { active hydrothermal activity }\end{array}$ & $\begin{array}{l}\text { Finger-like protrusion of } \\
\text { entirely fresh glass, no } \\
\text { visible alteration }\end{array}$ \\
\hline $\begin{array}{l}\mathrm{AlO} \\
\text { (EPR4059-B2) }\end{array}$ & $9^{\circ} 50^{\prime} 38^{\prime \prime}$ & $104^{\circ} 17^{\prime} 86^{\prime \prime}$ & 2516 & $\begin{array}{l}\text { Lobate lava flow } \sim 0.8 \mathrm{~km} \\
\text { west of the AST, no } \\
\text { evidence of current venting }\end{array}$ & $\begin{array}{l}\text { Glassy crust of basalt with } \\
\text { yellow-brown Al-oxide } \\
\text { staining, glass relatively } \\
\text { fresh }\end{array}$ \\
\hline $\begin{array}{l}\mathrm{FeO} 1 \\
\text { (EPR3965-I2) }\end{array}$ & $9^{\circ} 49^{\prime} 41^{\prime \prime}$ & $104^{\circ} 16^{\prime} 32^{\prime \prime}$ & 2546 & $\begin{array}{l}\text { Large pillow lava mound } \sim 1 \\
\mathrm{~km} \text { east of the AST, not } \\
\text { near venting }\end{array}$ & $\begin{array}{l}\text { Interior of basalt } \\
\text { (microcrystalline) with } \\
\text { prevalent Fe-oxide staining }\end{array}$ \\
\hline $\begin{array}{l}\mathrm{FeO} 2 \\
\text { (EPR3968-O8a) }\end{array}$ & $9^{\circ} 28^{\prime} 48^{\prime \prime}$ & $104^{\circ} 15^{\prime} 0^{\prime \prime}$ & 2674 & $\begin{array}{l}\text { Lobate lava flow } \sim 0.5 \mathrm{~km} \\
\text { west of the AST near } \\
\text { diffuse hydrothermal } \\
\text { venting }\end{array}$ & $\begin{array}{l}\text { Thick glassy crust on lava } \\
\text { with Fe-oxide staining on } \\
\text { surface and fractures, glass } \\
\text { shows slight alteration }\end{array}$ \\
\hline $\begin{array}{l}\mathrm{MnO} \\
\text { (EPR3970-MO1A) }\end{array}$ & $9^{\circ} 30^{\prime} 36^{\prime \prime}$ & $104^{\circ} 13^{\prime} 48^{\prime \prime}$ & 2612 & $\begin{array}{l}\text { Lobate lava flow } \sim 0.5 \mathrm{~km} \\
\text { east of the AST }\end{array}$ & $\begin{array}{l}\text { Thick ferromanganese oxide } \\
\text { crust coating basalt with a } \\
\text { thin, altered glassy layer }\end{array}$ \\
\hline $\begin{array}{l}\text { DSW } \\
\text { (EPR4055-N3) }\end{array}$ & $9^{\circ} 28^{\prime} 89^{\prime \prime}$ & $104^{\circ} 14^{\prime} 78^{\prime \prime}$ & 2572 & $\sim 0.5 \mathrm{~km}$ west of the AST & Deep Ocean Seawater \\
\hline
\end{tabular}

Table 3.2. qPCR analyses (modified after Chapter 1).

\begin{tabular}{|c|c|c|c|c|c|c|}
\hline \multirow[b]{2}{*}{ Sample Name } & \multicolumn{2}{|c|}{ 16S Gene Copies $\mathrm{g}^{-1}$ rock } & \multicolumn{2}{|c|}{ Cells $\mathrm{g}^{-1}$ rock } & \multirow[b]{2}{*}{$\begin{array}{c}\% \\
\text { Bacteria }\end{array}$} & \multirow[b]{2}{*}{$\begin{array}{c}\% \\
\text { Archaea }\end{array}$} \\
\hline & Bacteria & Archaea & Bacteria & Archaea & & \\
\hline FG & $1.13 \pm 0.09 \times 10^{7}$ & $6.09 \pm 0.71 \times 10^{5}$ & $2.76 \pm 0.23 \times 10^{6}$ & $3.80 \pm 0.45 \times 10^{5}$ & 87.87 & 12.13 \\
\hline $\mathrm{AlO}$ & $9.06 \pm 0.18 \times 10^{7}$ & $1.58 \pm 0.09 \times 10^{6}$ & $2.21 \pm 0.04 \times 10^{7}$ & $9.89 \pm 0.57 \times 10^{5}$ & 95.72 & 4.28 \\
\hline FeO1 & $\mathrm{NM}^{\mathrm{a}}$ & NM & NM & NM & $\mathrm{NM}$ & NM \\
\hline $\mathrm{FeO} 2$ & $2.36 \pm 0.18 \times 10^{8}$ & $6.69 \pm 0.23 \times 10^{6}$ & $5.77 \pm 0.43 \times 10^{7}$ & $4.18 \pm 0.15 \times 10^{6}$ & 93.23 & 6.77 \\
\hline $\mathrm{MnO}$ & $5.70 \pm 0.42 \times 10^{9}$ & $1.21 \pm 0.36 \times 10^{8}$ & $1.39 \pm 0.10 \times 10^{9}$ & $7.54 \pm 2.28 \times 10^{7}$ & 94.85 & 5.15 \\
\hline DSW & NM & NM & NM & NM & NM & $\mathrm{NM}$ \\
\hline
\end{tabular}


Table 3.3. Observed and shared richness between bacterial communities as determined by the Chaol and shared Chaol $\left(\mathrm{S}_{1,2}\right.$ Chao $)$ estimators, respectively.

\begin{tabular}{lcccccccc}
\hline & & \multicolumn{5}{c}{ Shared Richness $\left(\mathrm{S}_{1,2}\right.$ Chao } \\
\cline { 4 - 8 } Sample & Chao1 & $95 \%$ CI & FG & AlO & FeO1 & FeO2 & MnO & DSW \\
\hline FG & 84 & $56-157$ & NA & 6 & 23 & 7 & 14 & 7 \\
$\mathrm{AlO}$ & 74 & $53-131$ & & NA & 9 & 15 & 18 & 0 \\
$\mathrm{FeO} 1$ & 157 & $91-320$ & & & NA & 42 & 14 & 3 \\
$\mathrm{FeO} 2$ & 145 & $93-268$ & & & & $\mathrm{NA}$ & 40 & 0 \\
$\mathrm{MnO}$ & 153 & $96-290$ & & & & & NA & 0 \\
$\mathrm{DSW}$ & 12 & $11-20$ & & & & & & NA \\
\hline
\end{tabular}

Table 3.4. Bacterial community overlap $\left(\mathrm{J}_{\mathrm{abund}}\right)$ and structure $\left(\theta_{\mathrm{YC}}\right)$ similarity estimates.

\begin{tabular}{|c|c|c|c|c|c|c|c|c|c|c|c|c|}
\hline \multirow[b]{2}{*}{ Sample } & \multicolumn{2}{|c|}{ FG } & \multicolumn{2}{|r|}{$\mathrm{AlO}$} & \multicolumn{2}{|r|}{$\mathrm{FeO} 1$} & \multicolumn{2}{|r|}{$\mathrm{FeO} 2$} & \multicolumn{2}{|r|}{$\mathrm{MnO}$} & \multicolumn{2}{|r|}{ DSW } \\
\hline & $\mathrm{J}_{\text {abund }}$ & $\Theta_{\mathrm{YC}}$ & $J_{\text {abund }}$ & $\Theta_{\mathrm{YC}}$ & $\overline{J_{\text {abund }}}$ & $\Theta_{\mathrm{YC}}$ & $\overline{J_{\text {abund }}}$ & $\Theta_{\mathrm{YC}}$ & $\mathrm{J}_{\text {abund }}$ & $\Theta_{\mathrm{YC}}$ & $\overline{J_{\text {abund }}}$ & $\Theta_{\mathrm{YC}}$ \\
\hline FG & 1.00 & 1.00 & 0.04 & $0.02(0.01)$ & 0.20 & $0.10(0.05)$ & 0.10 & $0.10(0.04)$ & 0.09 & $0.04(0.02)$ & 0.17 & $0.07(0.03)$ \\
\hline $\mathrm{AlO}$ & & & 1.00 & 1.00 & 0.05 & $0.03(0.03)$ & 0.19 & $0.14(0.05)$ & 0.20 & $0.12(0.04)$ & 0.00 & 0.00 \\
\hline $\mathrm{FeO} 1$ & & & & & 1.00 & 1.00 & 0.24 & $0.65(0.04)$ & 0.08 & $0.06(0.04)$ & 0.03 & $0.07(0.01)$ \\
\hline $\mathrm{FeO} 2$ & & & & & & & 1.00 & 1.00 & 0.27 & $0.19(0.06)$ & 0.00 & 0.00 \\
\hline $\mathrm{MnO}$ & & & & & & & & & 1.00 & 1.00 & 0.00 & 0.00 \\
\hline DSW & & & & & & & & & & & 1.00 & 1.00 \\
\hline
\end{tabular}




\subsection{Figures}

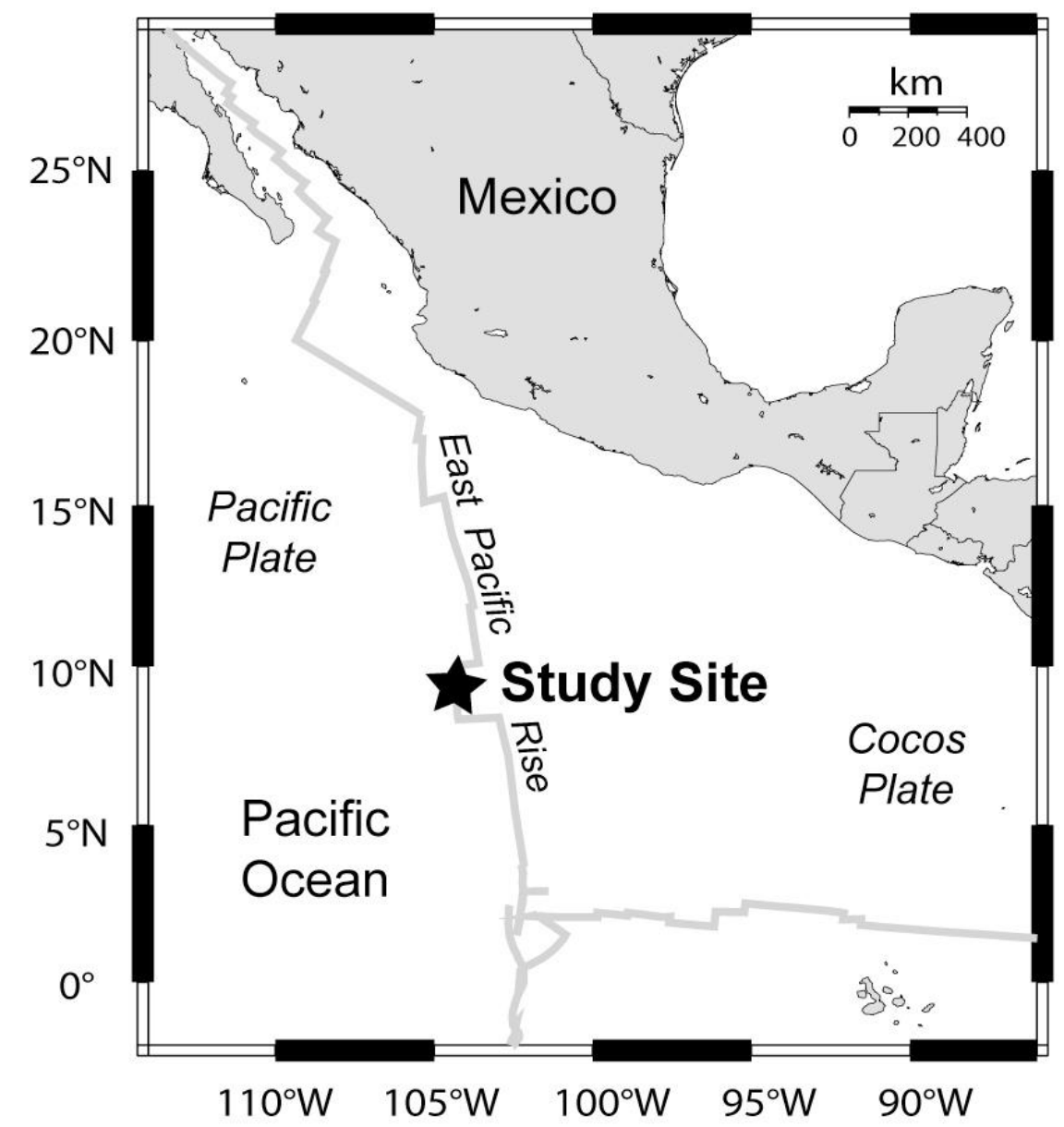

Figure 3.1. Map of the Eastern equatorial Pacific Ocean showing the study site location. Basaltic seafloor lavas were collected at or near the ridge-axis at the East Pacific Rise at $\sim 9^{\circ} \mathrm{N}$. 


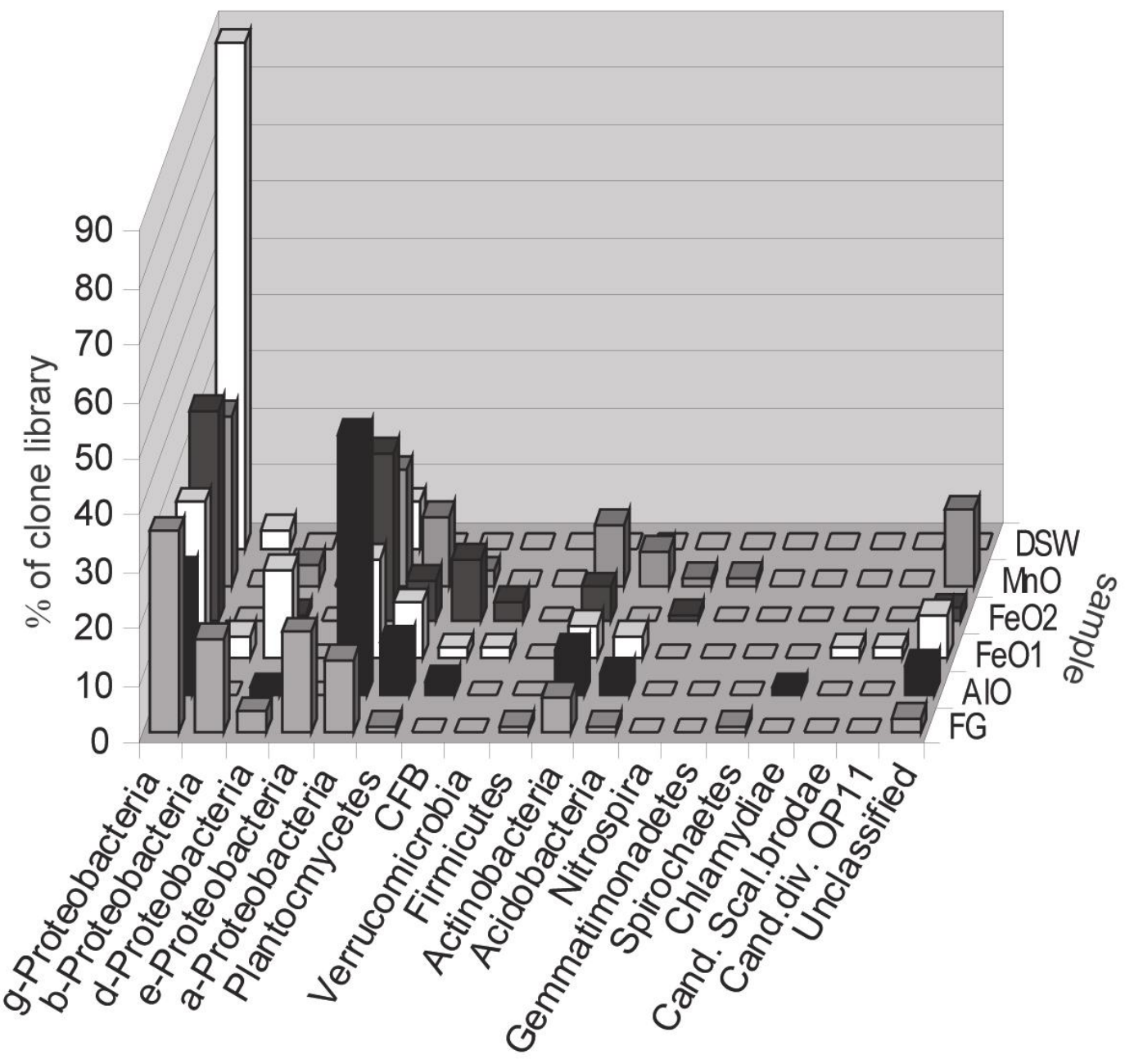

Figure 3.2. Chart showing the distribution of phyla recovered from each sample. Phylogenetic groups not represented in a specific clone library are denoted by unfilled boxes. 


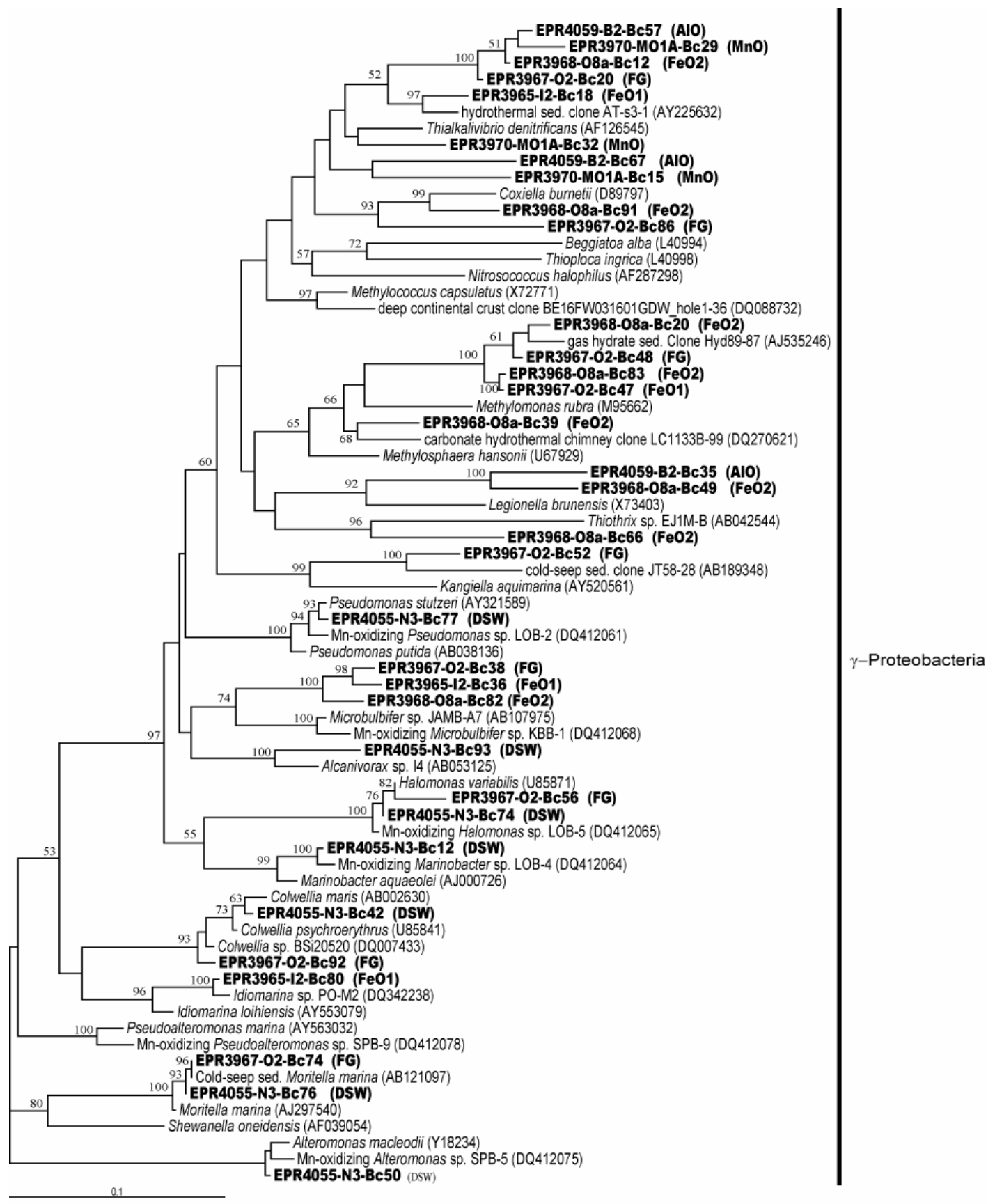

Figure 3.3. Phylogenetic trees showing the relationships of select $\gamma$-Proteobacteria sequences recovered from EPR basalts and deep seawater as determined by maximum likelihood analysis. Clones from this study are indicated in bold (full clone names as EPR Alvin dive \# - sample \# - bacterial clone \#, e.g. EPR3965-I2-Bc10) with their short sample indicator (e.g. FeO1). Bootstrap values for nodes with $>50 \%$ support, based on 500 replicates, are displayed as percentages. The scale bar represents 0.1 substitutions per nucleotide position. 


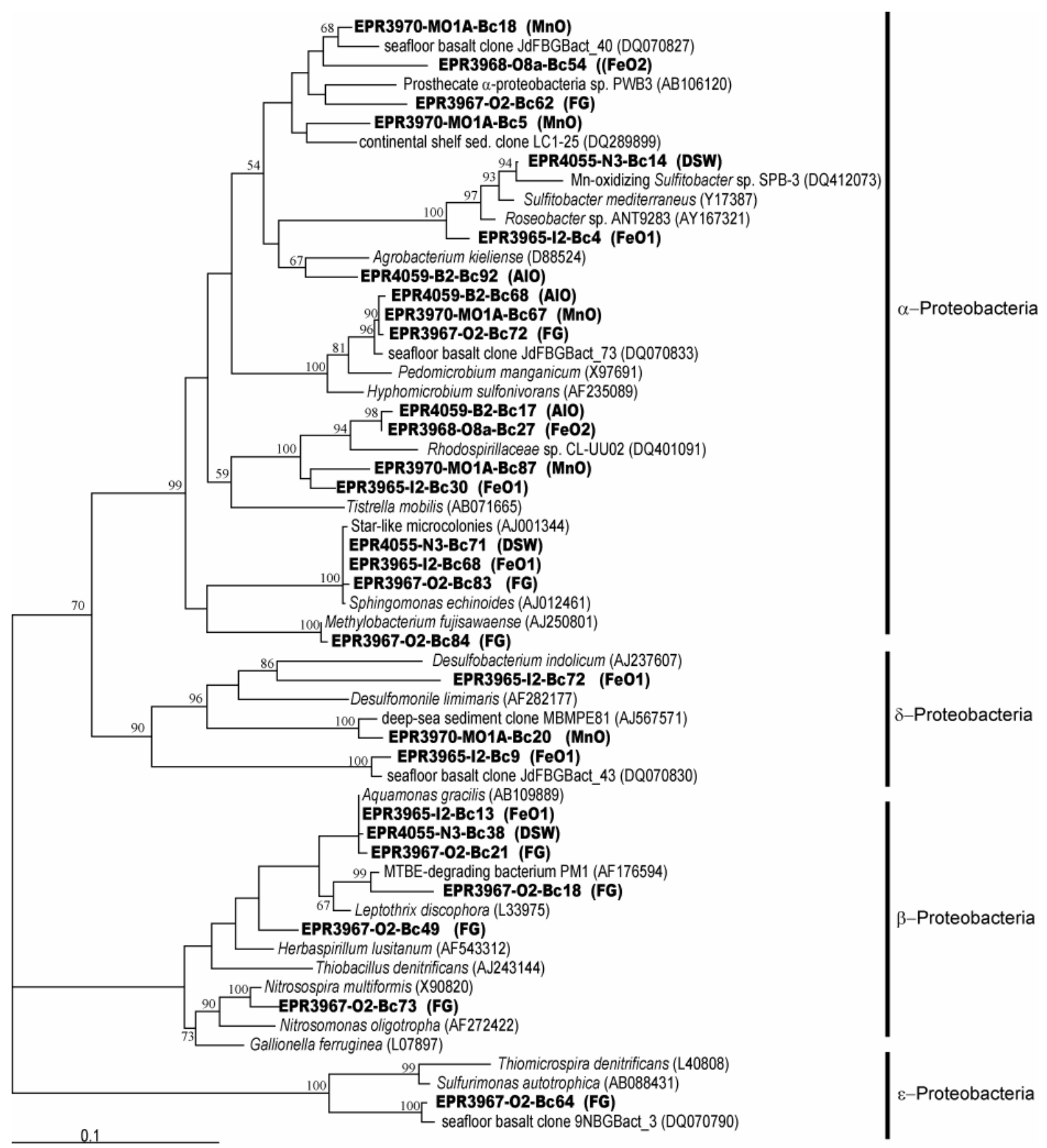

Figure 3.4. Phylogenetic trees showing the relationships of select $\alpha-, \beta-, \delta-$, and $\varepsilon$-Proteobacteria sequences recovered from EPR basalts and deep seawater as determined by maximum likelihood analysis. Clones from this study are indicated in bold with their short sample indicator. Bootstrap values for nodes with $>50 \%$ support, based on 500 replicates, are displayed as percentages. The scale bar represents 0.1 substitutions per nucleotide position. 


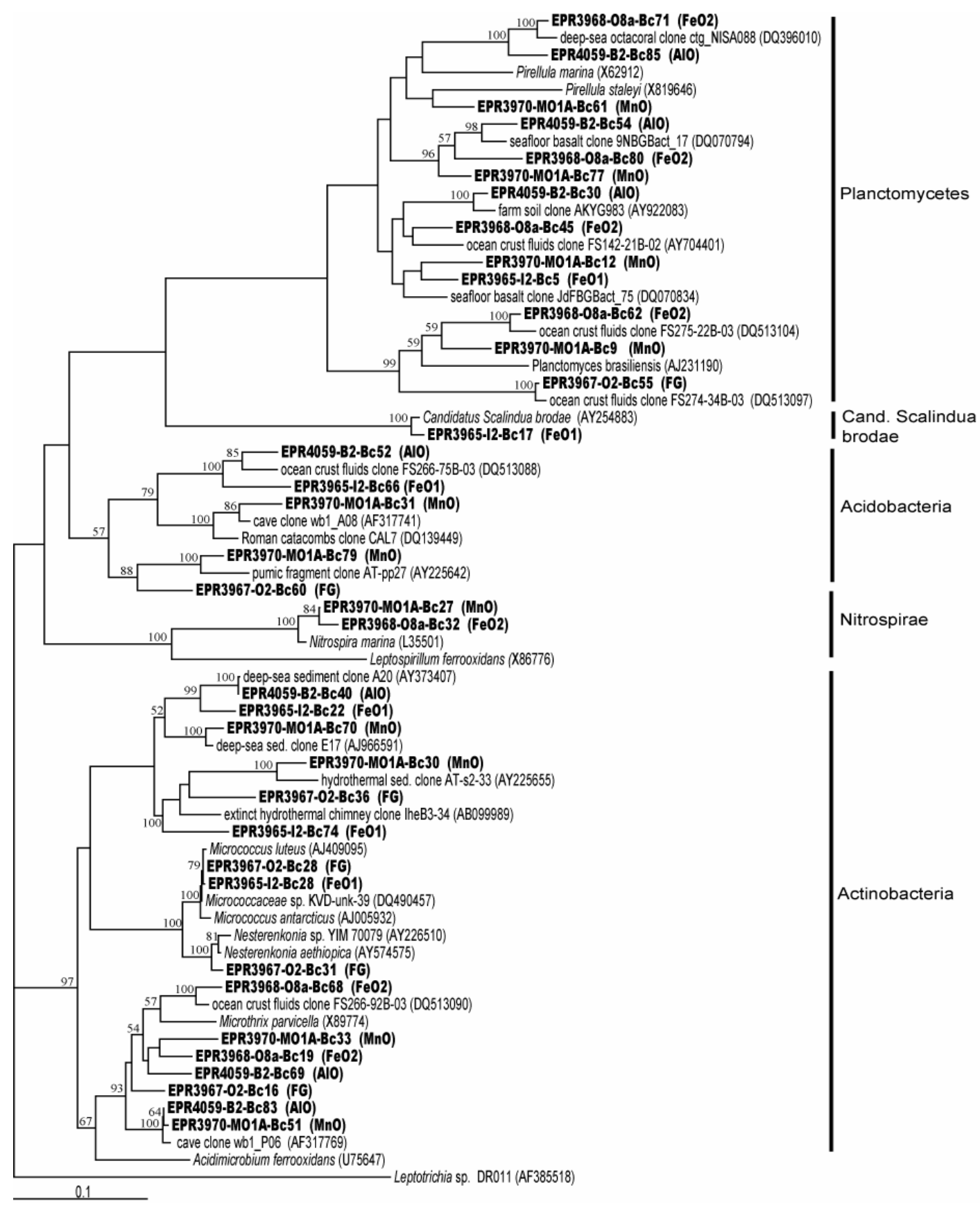

Figure 3.5. Phylogenetic trees showing the relationships of select non-Proteobacteria sequences (recovered from EPR basalts and deep seawater as determined by maximum likelihood analysis. Clones from this study are indicated in bold (full clone names as EPR Alvin dive \# - sample \# - bacterial clone \#, e.g. EPR3965-I2-Bc10) with their short sample indicator (e.g. FeO1). Bootstrap values for nodes with $>50 \%$ support, based on 1000 replicates, are displayed as percentages. The scale bar represents 0.1 substitutions per nucleotide position. 


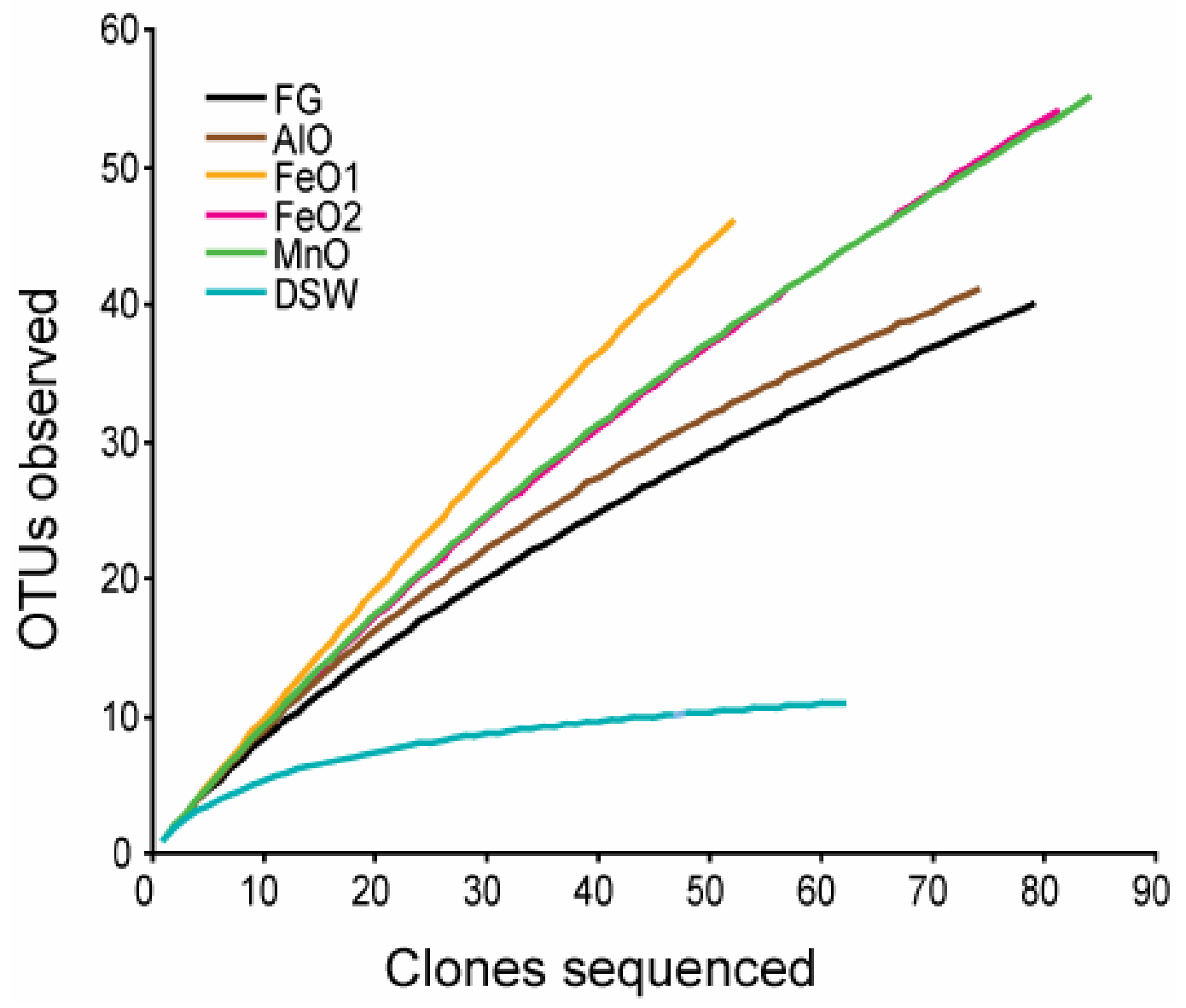

Figure 3.6. Rarefaction analysis comparing the relative diversity among the variably altered seafloor basalt samples and a deep seawater sample. 


\subsection{Appendix}

Table A1. Classification of all clones and closest BLAST match.

\begin{tabular}{|c|c|c|c|}
\hline Clone & $\begin{array}{l}\text { Phylogenetic } \\
\text { Affiliation }\end{array}$ & Closest 16S rRNA BLAST match (accession number) & $\begin{array}{c}\text { Identity } \\
(\%)\end{array}$ \\
\hline EPR3965-I2-Bc1 & delta-Proteobacteria & Uncultured delta proteobacterium clone JdFBGBact_10 (DQ070818) & 96 \\
\hline EPR3965-I2-Bc2 & delta-Proteobacteria & Uncultured bacterium clone Hast1_15 (DQ256632) & 94 \\
\hline EPR3965-I2-Bc4 & alpha-Proteobacteria & Uncultured bacterium clone B175 16S (AY375057) & 98 \\
\hline EPR3965-I2-Bc5 & Planctomycetes & Uncultured planctomycete clone JdFBGBact_75 (DQ070834) & 94 \\
\hline EPR3965-I2-Bc8 & beta-Proteobacteria & Curvibacter gracilis (AB109889) & 100 \\
\hline EPR3965-I2-Bc9 & delta-Proteobacteria & Uncultured delta proteobacterium clone JdFBGBact_43 (DQ070830) & 98 \\
\hline EPR3965-I2-Bc12 & Planctomycetes & Uncultured organism clone ctg_CGOAB92 (DQ395415) & 91 \\
\hline EPR3965-I2-Bc13 & beta-Proteobacteria & Curvibacter gracilis gene (AB109889) & 100 \\
\hline EPR3965-I2-Bc17 & Cand. Scalindua brodae & Candidatus Scalindua brodae clone EN 8 (AY254883) & 98 \\
\hline EPR3965-I2-Bc18 & gamma-Proteobacteria & Uncultured gamma proteobacterium clone AT-s3-1 (AY225632) & 96 \\
\hline EPR3965-I2-Bc19 & gamma-Proteobacteria & Uncultured gamma proteobacterium clone MBMPE11 (AJ567537) & 99 \\
\hline EPR3965-I2-Bc20 & Planctomycetes & Uncultured bacterium clone FS275-22B-03 (DQ513104) & 98 \\
\hline EPR3965-I2-Bc21 & gamma-Proteobacteria & $\begin{array}{l}\text { Uncultured gamma proteobacterium clone Belgica2005/10-120-14 } \\
\text { (DQ351746) }\end{array}$ & 97 \\
\hline EPR3965-I2-Bc22 & Actinobacteria & Uncultured bacterium clone Kazan-1B-42/BC19-1B-42 (AY592119) & 96 \\
\hline EPR3965-I2-Bc25 & alpha-Proteobacteria & Rickettsia canada (L36104) & 87 \\
\hline EPR3965-I2-Bc27 & Planctomyetes & Uncultured bacterium clone FS142-21B-02 (AY704401) & 93 \\
\hline EPR3965-I2-Bc28 & Actinobacteria & Micrococcaceae bacterium KVD-unk-39 (DQ490457) & 100 \\
\hline EPR3965-I2-Bc30 & alpha-Proteobacteria & Uncultured bacterium clone A56 (AY373412) & 97 \\
\hline EPR3965-I2-Bc32 & Verrucomicrobia & Uncultured marine bacterium clone SJC3.21 (DQ071119) & 97 \\
\hline EPR3965-I2-Bc34 & Planctomyetes & Uncultured soil bacterium clone UC2 (DQ298013) & 92 \\
\hline EPR3965-I2-Bc35 & delta-Proteobacteria & Uncultured delta proteobacterium clone EB1052 (AY395371) & 94 \\
\hline EPR3965-I2-Bc36 & gamma-Proteobacteria & Uncultured marine bacterium clone AntCL1D1 (DQ906720) & 96 \\
\hline EPR3965-I2-Bc38 & alpha-Proteobacteria & Uncultured organism clone ctg_CGOAA09 (DQ395453) & 99 \\
\hline EPR3965-I2-Bc40 & Cand. division OP11 & $\begin{array}{l}\text { Uncultured candidate division OP11 bacterium MERTZ_21CM_109 } \\
\text { (AF424448) }\end{array}$ & 92 \\
\hline EPR3965-I2-Bc43 & Unidentified & Uncultured bacterium clone CV106 (DQ499326) & 92 \\
\hline EPR3965-I2-Bc45 & Unidentified & Uncultured bacterium clone AKYG548 (AY921936) & 95 \\
\hline EPR3965-I2-Bc47 & gamma-Proteobacteria & $\begin{array}{l}\text { Uncultured gamma proteobacterium clone Belgica2005/10-140-24 } \\
\text { (DQ351796) }\end{array}$ & 98 \\
\hline EPR3965-I2-Bc49 & gamma-Proteobacteria & Marine gamma proteobacterium HTCC2180 (AY386343) & 97 \\
\hline EPR3965-I2-Bc51 & Unidentified & Uncultured bacterium partial (AJ966584) & 93 \\
\hline EPR3965-I2-Bc52 & CFB group & Uncultured Cytophaga clone: BD2-17 (AB015545) & 99 \\
\hline EPR3965-I2-Bc55 & Unidentified & Uncultured Verrucomicrobia bacterium clone Nubeena196 (AY50005£ & 93 \\
\hline EPR3965-I2-Bc56 & gamma-Proteobacteria & $\begin{array}{l}\text { Uncultured gamma proteobacterium clone Belgica2005/10-130-12 } \\
\text { (DQ351758) }\end{array}$ & 97 \\
\hline EPR3965-I2-Bc59 & gamma-Proteobacteria & Uncultured gamma proteobacterium clone DS036 (DQ234120) & 92 \\
\hline EPR3965-I2-Bc64 & alpha-Proteobacteria & Uncultured organism clone ctg_CGOAA09 (DQ395453) & 100 \\
\hline EPR3965-I2-Bc65 & gamma-Proteobacteria & Uncultured proteobacterium clone SIMO-2184 (AY711550) & 98 \\
\hline EPR3965-I2-Bc66 & Acidobacteria & Uncultured Acidobacteria clone JG36-GS-72 (AJ582048) & 97 \\
\hline EPR3965-I2-Bc67 & alpha-Proteobacteria & Uncultured alpha proteobacteriumclone MBMPE50 (AJ567563) & 96 \\
\hline EPR3965-I2-Bc68 & alpha-Proteobacteria & Sphingomonas sp. oral clone AV069 (AF385529) & 100 \\
\hline EPR3965-I2-Bc69 & gamma-Proteobacteria & $\begin{array}{l}\text { Uncultured gamma proteobacterium clone Belgica2005/10-140-1 } \\
\text { (DQ351774) }\end{array}$ & 93 \\
\hline EPR3965-I2-Bc72 & delta-Proteobacteria & Uncultured bacterium clone s2uc28 (DQ416322) & 96 \\
\hline EPR3965-I2-Bc73 & gamma-Proteobacteria & Uncultured gamma proteobacterium clone AT-s3-1 (AY225632) & 96 \\
\hline EPR3965-I2-Bc74 & Actinobacteria & Uncultured bacterium clone Kazan-1B-43/BC19-1B-43 (AY592120) & 97 \\
\hline EPR3965-I2-Bc75 & Unidentified & Uncultured organism clone ctg_BRRAA88 (DQ395368) & 99 \\
\hline EPR3965-I2-Bc80 & gamma-Proteobacteria & Idiomarina sp. PO-M2 (DQ342238) & 98 \\
\hline EPR3965-I2-Bc82 & delta-Proteobacteria & Uncultured delta proteobacterium clone HMMVPog-8 (AJ704690) & 94 \\
\hline EPR3965-I2-Bc83 & alpha-Proteobacteria & Uncultured alpha proteobacterium clone AT-s3-44 (AY225603) & 98 \\
\hline
\end{tabular}




\begin{tabular}{|c|c|c|c|}
\hline Clone & $\begin{array}{l}\text { Phylogenetic } \\
\text { Affiliation }\end{array}$ & Closest 16S rRNA BLAST match (accession number) & $\begin{array}{c}\text { Identity } \\
(\%)\end{array}$ \\
\hline EPR3965-I2-Bc84 & gamma-Proteobacteria & $\begin{array}{l}\text { Uncultured gamma proteobacterium clone Belgica2005/10-130-4 } \\
\text { (DQ351753) }\end{array}$ & 95 \\
\hline EPR3965-I2-Bc85 & alpha-Proteobacteria & Uncultured alpha proteobacteriumnclone MBMPE50 (AJ567563) & 97 \\
\hline EPR3965-I2-Bc87 & delta-Proteobacteria & Uncultured delta proteobacterium clone JdFBGBact_10 (DQ070818) & 94 \\
\hline EPR3965-I2-Bc89 & gamma-Proteobacteria & $\begin{array}{l}\text { Uncultured gamma proteobacterium clone Belgica2005/10-130-4 } \\
\text { (DQ351753) }\end{array}$ & 95 \\
\hline EPR3965-I2-Bc90 & delta-Proteobacteria & Uncultured delta proteobacterium clone AKYH1423 (AY921676) & 90 \\
\hline EPR3967-O2-Bc1 & gamma-Proteobacteria & Uncultured gamma proteobacterium clone Hyd89-87 (AJ535246) & 97 \\
\hline EPR3967-O2-Bc3 & epsilon-Proteobacteria & Uncultured epsilon proteobacterium clone 9NBGBact_3 (DQ070790) & 100 \\
\hline EPR3967-O2-Bc6 & beta-Proteobacteria & Curvibacter gracilis (AB109889) & 100 \\
\hline $\begin{array}{l}\text { EPR3967-O2-Bc7 } \\
\text { EPR3967-O2-Bc8 }\end{array}$ & $\begin{array}{l}\text { delta-Proteobacteria } \\
\text { epsilon-Proteobacteria }\end{array}$ & $\begin{array}{l}\text { Uncultured delta proteobacterium clone JdFBGBact_10 (DQ070818) } \\
\text { Uncultured epsilon proteobacterium clone 9NBGBact_3 (DQ070790) }\end{array}$ & $\begin{array}{c}94 \\
100\end{array}$ \\
\hline EPR3967-O2-Bc9 & gamma-Proteobacteria & Halomonas sp. IB-559 (AJ309560) & 99 \\
\hline EPR3967-O2-Bc10 & beta-Proteobacteria & Curvibacter gracilis (AB109889) & 99 \\
\hline EPR3967-O2-Bc11 & beta-Proteobacteria & Curvibacter gracilis (AB109889) & 100 \\
\hline EPR3967-O2-Bc12 & beta-Proteobacteria & Curvibacter gracilis (AB109889) & 100 \\
\hline EPR3967-O2-Bc14 & Spirochaetes & Uncultured spirochete clone SIMO-2146 (AY711512) & 90 \\
\hline EPR3967-O2-Bc15 & delta-Proteobacteria & Uncultured bacterium isolate JH10_C65 (AY568817) & 92 \\
\hline EPR3967-O2-Bc16 & Actinobacteria & Uncultured organism clone ctg_CGOAA22 (DQ395502) & 94 \\
\hline EPR3967-O2-Bc18 & beta-Proteobacteria & Uncultured bacterium DSSD55 (AY328753) & 99 \\
\hline EPR3967-O2-Bc19 & gamma-Proteobacteria & $\begin{array}{l}\text { Uncultured gamma proteobacterium clone Belgica2005/10-120-6 } \\
\text { (DQ351741) }\end{array}$ & 97 \\
\hline EPR3967-O2-Bc20 & gamma-Proteobacteria & $\begin{array}{l}\text { Uncultured gamma proteobacterium clone Belgica2005/10-120-9 } \\
\text { (DQ351744) }\end{array}$ & 99 \\
\hline EPR3967-O2-Bc21 & beta-Proteobacteria & Curvibacter gracilis (AB109889) & 100 \\
\hline EPR3967-O2-Bc23 & gamma-Proteobacteria & Uncultured Methylobacter clone: JT58-28 (AB189348) & 94 \\
\hline EPR3967-O2-Bc24 & gamma-Proteobacteria & $\begin{array}{l}\text { Uncultured gamma proteobacterium clone Belgica2005/10-140-20 } \\
\text { (DQ351793) }\end{array}$ & 97 \\
\hline EPR3967-O2-Bc25 & gamma-Proteobacteria & Uncultured gamma proteobacterium clone Hyd89-87 (AJ535246) & 97 \\
\hline EPR3967-O2-Bc26 & alpha-Proteobacteria & $\begin{array}{l}\text { Uncultured Methylobacteriaceae bacterium clone 10-3Ba02 } \\
\text { (AY360526) }\end{array}$ & 100 \\
\hline EPR3967-O2-Bc27 & beta-Proteobacteria & Uncultured bacterium DSSD55 (AY328753) & 99 \\
\hline EPR3967-O2-Bc28 & Actinobacteria & Micrococcaceae bacterium KVD-unk-39 (DQ490457) & 99 \\
\hline EPR3967-O2-Bc29 & alpha-Proteobacteria & alpha proteobacterium endosymbion clone Ileu1-2-I-8 (AJ890099) & 94 \\
\hline EPR3967-O2-Bc30 & Unidentified & Uncultured bacterium clone HF130_A6_P1 (DQ300591) & 91 \\
\hline EPR3967-O2-Bc31 & Actinobacteria & Nesterenkonia abyssinica (AY574575) & 98 \\
\hline EPR3967-O2-Bc32 & gamma-Proteobacteria & Uncultured Methylobacter clone: JT58-28 (AB189348) & 93 \\
\hline EPR3967-O2-Bc33 & alpha-Proteobacteria & Uncultured alpha proteobacterium clone LiUU-9-26 (AY509403) & 90 \\
\hline EPR3967-O2-Bc34 & alpha-Proteobacteria & Uncultured bacterium clone PDM-OTU5 (AY700635) & 99 \\
\hline EPR3967-O2-Bc35 & beta-Proteobacteria & Curvibacter gracilis (AB109889) & 100 \\
\hline EPR3967-O2-Bc36 & Actinobacteria & Uncultured bacterium clone Kazan-2B-20/BC19-2B-20 (AY592144) & 92 \\
\hline EPR3967-O2-Bc38 & gamma-Proteobacteria & Uncultured marine bacterium clone AntCL1D1 (DQ906720) & 97 \\
\hline EPR3967-O2-Bc39 & gamma-Proteobacteria & $\begin{array}{l}\text { Uncultured gamma proteobacterium clone Belgica2005/10-140-5 } \\
\text { (DQ351778) }\end{array}$ & 99 \\
\hline EPR3967-O2-Bc40 & beta-Proteobacteria & Curvibacter gracilis (AB109889) & 100 \\
\hline EPR3967-O2-Bc41 & Unidentified & Uncultured Clostridiales bacterium clone: MgMjD-018 (AB234447) & 86 \\
\hline EPR3967-O2-Bc43 & gamma-Proteobacteria & Uncultured gamma proteobacterium clone: BD2-13 (AB015541) & 95 \\
\hline EPR3967-O2-Bc44 & epsilon-Proteobacteria & Uncultured epsilon proteobacterium clone 9NBGBact_3 (DQ070790) & 99 \\
\hline EPR3967-O2-Bc45 & epsilon-Proteobacteria & Uncultured epsilon proteobacterium clone 9NBGBact_3 (DQ070790) & 100 \\
\hline EPR3967-O2-Bc46 & gamma-Proteobacteria & $\begin{array}{l}\text { Uncultured gamma proteobacterium clone Belgica2005/10-140-24 } \\
\text { (DQ351796) }\end{array}$ & 96 \\
\hline EPR3967-O2-Bc47 & gamma-Proteobacteria & Uncultured bacterium clone SZB76 (AM176844) & 97 \\
\hline EPR3967-O2-Bc48 & gamma-Proteobacteria & Uncultured hydrocarbon seep bacterium BPC036 (AF154089) & 97 \\
\hline EPR3967-O2-Bc49 & beta-Proteobacteria & Uncultured bacterium clone: RB041 (AB240293) & 99 \\
\hline EPR3967-O2-Bc51 & beta-Proteobacteria & Acidovorax sp. 'smarlab 133815' (AY093698) & 99 \\
\hline
\end{tabular}




\begin{tabular}{|c|c|c|c|}
\hline Clone & $\begin{array}{l}\text { Phylogenetic } \\
\text { Affiliation }\end{array}$ & Closest 16S rRNA BLAST match (accession number) & $\begin{array}{c}\text { Identity } \\
(\%)\end{array}$ \\
\hline EPR3967-O2-Bc52 & gamma-Proteobacteria & Uncultured Methylobacter clone: JT58-28 (AB189348) & 94 \\
\hline EPR3967-O2-Bc53 & Actinobacteria & Uncultured organism clone ctg_CGOAA22 (DQ395502) & 94 \\
\hline EPR3967-O2-Bc54 & beta-Proteobacteria & Uncultured bacterium DSSD55 (AY328753) & 99 \\
\hline EPR3967-O2-Bc55 & Planctomycetes & Uncultured bacterium clone FS274-34B-03 (DQ513097) & 99 \\
\hline EPR3967-O2-Bc56 & gamma-Proteobacteria & Uncultured organism clone ctg_NISA373 (DQ396023) & 100 \\
\hline EPR3967-O2-Bc57 & delta-Proteobacteria & Uncultured bacterium clone FS266-91B-03 (AY869686) & 96 \\
\hline EPR3967-O2-Bc58 & alpha-Proteobacteria & Kordiimonas gwangyangensis strain GW14-5 (AY682384) & 90 \\
\hline EPR3967-O2-Bc59 & epsilon-Proteobacteria & Uncultured epsilon proteobacterium clone 9NBGBact_3 (DQ070790) & 100 \\
\hline EPR3967-O2-Bc60 & Acidobacteria & uncultured Holophaga/Acidobacterium Sva0515 (AJ241004) & 95 \\
\hline EPR3967-O2-Bc62 & alpha-Proteobacteria & $\begin{array}{l}\text { Uncultured alpha proteobacterium clone Belgica2005/10-140-4 } \\
\text { (DQ351777) }\end{array}$ & 93 \\
\hline EPR3967-O2-Bc64 & epsilon-Proteobacteria & Uncultured epsilon proteobacterium clone 9NBGBact_3 (DQ070790) & 99 \\
\hline EPR3967-O2-Bc65 & gamma-Proteobacteria & $\begin{array}{l}\text { Uncultured gamma proteobacterium clone Belgica2005/10-140-8 } \\
\text { (DQ351781) }\end{array}$ & 95 \\
\hline EPR3967-O2-Bc66 & gamma-Proteobacteria & Uncultured gamma proteobacterium clone:JTB254 (AB015253) & 97 \\
\hline EPR3967-O2-Bc67 & epsilon-Proteobacteria & Uncultured epsilon proteobacterium clone 9NBGBact_3 (DQ070790) & 100 \\
\hline EPR3967-O2-Bc68 & gamma-Proteobacteria & $\begin{array}{l}\text { Uncultured gamma proteobacterium clone Belgica2005/10-140-24 } \\
\text { (DQ351796) }\end{array}$ & 97 \\
\hline EPR3967-O2-Bc69 & epsilon-Proteobacteria & Uncultured epsilon proteobacterium clone 9NBGBact_3 (DQ070790) & 100 \\
\hline $\begin{array}{l}\text { EPR3967-O2-Bc70 } \\
\text { EPR3967-O2-Bc71 }\end{array}$ & $\begin{array}{l}\text { epsilon-Proteobacteria } \\
\text { gamma-Proteobacteria }\end{array}$ & $\begin{array}{l}\text { Uncultured epsilon proteobacterium clone 9NBGBact_3 (DQ070790) } \\
\text { Oligobrachia mashikoi endosymbiont (AB252051) }\end{array}$ & $\begin{array}{l}99 \\
96\end{array}$ \\
\hline EPR3967-O2-Bc72 & Firmicutes & Thermalkalibacillus uzonensis (DQ221694) & 98 \\
\hline EPR3967-O2-Bc73 & beta-Proteobacteria & Uncultured bacterium clone: BS169 (AB240278) & 97 \\
\hline EPR3967-O2-Bc74 & gamma-Proteobacteria & Moritella marina (AB121097) & 100 \\
\hline EPR3967-O2-Bc75 & epsilon-Proteobacteria & Uncultured epsilon proteobacterium clone 9NBGBact_3 (DQ070790) & 100 \\
\hline EPR3967-O2-Bc77 & gamma-Proteobacteria & Uncultured gamma proteobacterium, clone: BD3-1 (AB015547) & 90 \\
\hline EPR3967-O2-Bc78 & gamma-Proteobacteria & Uncultured gamma proteobacterium clone Hyd89-87 (AJ535246) & 97 \\
\hline EPR3967-O2-Bc79 & gamma-Proteobacteria & Uncultured bacterium clone SZB76 (AM176844 & 97 \\
\hline EPR3967-O2-Bc80 & epsilon-Proteobacteria & Uncultured epsilon proteobacterium clone 9NBGBact_3 (DQ070790) & 99 \\
\hline EPR3967-O2-Bc81 & alpha-Proteobacteria & Uncultured organism clone ctg_CGOAB55 (DQ395481) & 97 \\
\hline EPR3967-O2-Bc82 & epsilon-Proteobacteria & Uncultured epsilon proteobacterium clone 9NBGBact_3 (DQ070790) & 100 \\
\hline EPR3967-O2-Bc83 & alpha-Proteobacteria & Sphingomonas sp. oral clone AV069 (AF385529) & 100 \\
\hline EPR3967-O2-Bc84 & alpha-Proteobacteria & $\begin{array}{l}\text { Uncultured Methylobacteriaceae bacterium clone } 10-3 \mathrm{Ba} 02 \\
\text { (AY360526) }\end{array}$ & 99 \\
\hline EPR3967-O2-Bc85 & gamma-Proteobacteria & Colwellia sp. BSi20520 (DQ007433) & 98 \\
\hline EPR3967-O2-Bc86 & gamma-Proteobacteria & Uncultured gamma proteobacterium clone:JTB256 (AB015255) & 91 \\
\hline EPR3967-O2-Bc88 & epsilon-Proteobacteria & Uncultured epsilon proteobacterium clone 9NBGBact_3 (DQ070790) & 99 \\
\hline EPR3967-O2-Bc89 & alpha-Proteobacteria & Uncultured organism clone ctg_CGOAB55 (DQ395481) & 96 \\
\hline EPR3967-O2-Bc90 & gamma-Proteobacteria & Oligobrachia mashikoi endosymbiont (AB252051) & 96 \\
\hline EPR3967-O2-Bc91 & epsilon-Proteobacteria & Uncultured epsilon proteobacterium clone 9NBGBact_3 (DQ070790) & 100 \\
\hline EPR3967-O2-Bc92 & gamma-Proteobacteria & Colwellia sp. BSi20520 (DQ007433) & 98 \\
\hline EPR3968-O8a-Bc1 & Verrucomicrobia & Uncultured bacterium clone CV35 (DQ499289) & 94 \\
\hline EPR3968-O8a-Bc2 & gamma-Proteobacteria & Uncultured bacterium clone LC1133B-99 (DQ270621) & 95 \\
\hline EPR3968-O8a-Bc3 & gamma-Proteobacteria & $\begin{array}{l}\text { Uncultured gamma proteobacterium clone Belgica2005/10-130-9 } \\
\text { (DQ351756) }\end{array}$ & 96 \\
\hline EPR3968-O8a-Bc5 & gamma-Proteobacteria & Uncultured gamma proteobacterium clone Hyd89-87 (AJ535246) & 97 \\
\hline EPR3968-O8a-Bc6 & gamma-Proteobacteria & $\begin{array}{l}\text { Uncultured gamma proteobacterium clone Belgica2005/10-140-5 } \\
\text { (DQ351778) }\end{array}$ & 99 \\
\hline EPR3968-O8a-Bc7 & gamma-Proteobacteria & Uncultured gamma proteobacterium clone MBMPE13 (AJ567539) & 99 \\
\hline EPR3968-O8a-Bc8 & Unidentified & Uncultured organism clone ctg_CGOCA20 (DQ395515) & 98 \\
\hline EPR3968-O8a-Bc10 & gamma-Proteobacteria & Uncultured bacterium clone LC1133B-99 (DQ270621) & 95 \\
\hline EPR3968-O8a-Bc11 & alpha-Proteobacteria & Uncultured organism clone ctg_CGOAB13 (DQ395499) & 95 \\
\hline EPR3968-O8a-Bc12 & gamma-Proteobacteria & $\begin{array}{l}\text { Uncultured gamma proteobacterium clone Belgica2005/10-120-9 } \\
\text { (DQ351744) }\end{array}$ & 98 \\
\hline EPR3968-O8a-Bc13 & gamma-Proteobacteria & Uncultured gamma proteobacterium clone:JTB255 (AB015254) & 97 \\
\hline
\end{tabular}




\begin{tabular}{|c|c|c|c|}
\hline Clone & $\begin{array}{l}\text { Phylogenetic } \\
\text { Affiliation }\end{array}$ & Closest 16S rRNA BLAST match (accession number) & $\begin{array}{c}\text { Identity } \\
(\%)\end{array}$ \\
\hline EPR3968-O8a-Bc14 & gamma-Proteobacteria & Uncultured bacterium clone SZB76 (AM176844) & 97 \\
\hline EPR3968-O8a-Bc15 & alpha-Proteobacteria & Uncultured organism clone ctg_CGOAB13 (DQ395499) & 95 \\
\hline EPR3968-O8a-Bc16 & gamma-Proteobacteria & Uncultured gamma proteobacterium clone AT-s75 (AY225637) & 97 \\
\hline EPR3968-O8a-Bc17 & gamma-Proteobacteria & $\begin{array}{l}\text { Uncultured gamma proteobacterium clone Belgica2005/10-120-9 } \\
\text { (DQ351744) }\end{array}$ & 98 \\
\hline EPR3968-O8a-Bc18 & gamma-Proteobacteria & Uncultured bacterium clone SZB76 (AM176844) & 97 \\
\hline EPR3968-O8a-Bc19 & Actinobacteria & Uncultured marine bacterium (DQ071068) & 97 \\
\hline EPR3968-O8a-Bc20 & gamma-Proteobacteria & Uncultured gamma proteobacterium clone Hyd89-87 (AJ535246) & 97 \\
\hline EPR3968-O8a-Bc21 & gamma-Proteobacteria & Uncultured gamma proteobacterium clone:JTB255 (AB015254) & 98 \\
\hline EPR3968-O8a-Bc22 & Verrucomicrobia & Uncultured Verrucomicrobia bacterium clone LD1-PB3 (AY114335) & 93 \\
\hline EPR3968-O8a-Bc23 & CFB group & Formosa algae clone SE60 (AY771766) & 95 \\
\hline EPR3968-O8a-Bc24 & alpha-Proteobacteria & Uncultured bacterium clone Napoli-1B-13 (AY592570) & 98 \\
\hline EPR3968-O8a-Bc25 & gamma-Proteobacteria & Uncultured bacterium clone LC1133B-99 (DQ270621) & 95 \\
\hline EPR3968-O8a-Bc27 & alpha-Proteobacteria & Mucus bacterium 23 (AY654769) & 95 \\
\hline EPR3968-O8a-Bc28 & gamma-Proteobacteria & Uncultured gamma proteobacterium clone AT-s3-1 (AY225632) & 91 \\
\hline EPR3968-O8a-Bc30 & alpha-Proteobacteria & Uncultured alpha proteobacterium clone LC1-25 (DQ289899) & 93 \\
\hline EPR3968-O8a-Bc31 & alpha-Proteobacteria & Uncultured alpha proteobacterium clone:JTB359 (AB015247) & 98 \\
\hline EPR3968-O8a-Bc32 & Nitrospirae & Uncultured organism clone ctg_CGOAB70 (DQ395470) & 99 \\
\hline EPR3968-O8a-Bc34 & CFB group & Mucus bacterium 117 (AY654753) & 96 \\
\hline EPR3968-O8a-Bc36 & alpha-Proteobacteria & Uncultured bacterium clone SZB47 (AM176849) & 97 \\
\hline EPR3968-O8a-Bc37 & alpha-Proteobacteria & Uncultured alpha proteobacterium clone LC1-25 (DQ289899) & 92 \\
\hline EPR3968-O8a-Bc38 & alpha-Proteobacteria & $\begin{array}{l}\text { Uncultured alpha proteobacterium clone Belgica2005/10-140-4 } \\
\text { (DQ351777) }\end{array}$ & 99 \\
\hline EPR3968-O8a-Bc39 & gamma-Proteobacteria & Uncultured bacterium clone LC1133B-99 (DQ270621) & 95 \\
\hline EPR3968-O8a-Bc41 & CFB group & Uncultured Cytophaga clone: JT75-329 (AB189382) & 96 \\
\hline EPR3968-O8a-Bc42 & gamma-Proteobacteria & $\begin{array}{l}\text { Uncultured gamma proteobacterium clone Belgica2005/10-140-5 } \\
\text { (DQ351778) }\end{array}$ & 98 \\
\hline EPR3968-O8a-Bc43 & gamma-Proteobacteria & Uncultured gamma proteobacterium clone JdFBGBact_18 (DQ070821 & 100 \\
\hline EPR3968-O8a-Bc44 & Actinobacteria & Uncultured bacterium clone CTD005-31B-02 (DQ513065) & 94 \\
\hline EPR3968-O8a-Bc45 & Planctomycetes & Uncultured organism clone ctg_CGOAB92 (DQ395415) & 94 \\
\hline EPR3968-O8a-Bc46 & CFB group & Uncultured Bacteroidetes bacterium clone Nubeena127 (AY500022) & 99 \\
\hline EPR3968-O8a-Bc47 & alpha-Proteobacteria & Uncultured organism clone ctg_CGOAB13 (DQ395499) & 94 \\
\hline EPR3968-O8a-Bc48 & Verrucomicrobia & Uncultured Verrucomicrobia Arctic95D-9 (AY028220) & 94 \\
\hline EPR3968-O8a-Bc49 & gamma-Proteobacteria & Uncultured bacterium clone DBBB-38 (AY226290) & 95 \\
\hline EPR3968-O8a-Bc51 & alpha-Proteobacteria & Uncultured bacterium clone G74 (DQ480486) & 94 \\
\hline EPR3968-O8a-Bc52 & CFB group & Bacteroidetes bacterium O-014 (DQ812543) & 94 \\
\hline EPR3968-O8a-Bc53 & alpha-Proteobacteria & Uncultured organism clone ctg_CGOAB13 (DQ395499) & 94 \\
\hline EPR3968-O8a-Bc54 & alpha-Proteobacteria & Uncultured alpha proteobacterium clone LC1-25 (DQ289899) & 92 \\
\hline EPR3968-O8a-Bc55 & gamma-Proteobacteria & Uncultured gamma proteobacterium clone:JTB255 (AB015254) & 99 \\
\hline EPR3968-O8a-Bc56 & alpha-Proteobacteria & Uncultured bacterium clone G74 (DQ480486) & 95 \\
\hline EPR3968-O8a-Bc57 & Planctomycetes & Uncultured planctomycete MERTZ_21CM_21 (AF424479) & 93 \\
\hline EPR3968-O8a-Bc58 & alpha-Proteobacteria & Uncultured Hyphomicrobium sp. clone JdFBGBact_73 (DQ070833) & 99 \\
\hline EPR3968-O8a-Bc59 & CFB group & Uncultured Lewinella clone: BJS81-142 (AB239037) & 98 \\
\hline EPR3968-O8a-Bc60 & Actinobacteria & Uncultured organism clone ctg_CGOAA79 (DQ395467) & 97 \\
\hline EPR3968-O8a-Bc61 & gamma-Proteobacteria & $\begin{array}{l}\text { Uncultured bacterium clone BE16FW031601GDW_hole1-36 } \\
\text { (DQ088732) }\end{array}$ & 89 \\
\hline EPR3968-O8a-Bc62 & Planctomycetes & Uncultured bacterium clone FS275-22B-03 (DQ513104) & 98 \\
\hline EPR3968-O8a-Bc64 & CFB group & Uncultured marine bacterium clone PB1.23 (DQ071072) & 95 \\
\hline EPR3968-O8a-Bc65 & alpha-Proteobacteria & Uncultured alpha proteobacterium clone LC1-25 (DQ289899) & 93 \\
\hline EPR3968-O8a-Bc66 & gamma-Proteobacteria & Uncultured bacterium clone LC1133B-23 (DQ270615) & 94 \\
\hline EPR3968-O8a-Bc67 & delta-Proteobacteria & Uncultured bacterium clone FS142-22B-02 (DQ513027) & 93 \\
\hline EPR3968-O8a-Bc68 & Actinobacteria & Uncultured bacterium clone FS266-92B-03 (DQ513090) & 97 \\
\hline EPR3968-O8a-Bc70 & gamma-Proteobacteria & Uncultured gamma proteobacterium clone:JTB256 (AB015255) & 93 \\
\hline
\end{tabular}




\begin{tabular}{|c|c|c|c|}
\hline Clone & $\begin{array}{l}\text { Phylogenetic } \\
\text { Affiliation }\end{array}$ & Closest 16S rRNA BLAST match (accession number) & $\begin{array}{c}\text { Identity } \\
(\%)\end{array}$ \\
\hline EPR3968-O8a-Bc71 & Planctomycetes & Uncultured organism clone ctg_NISA088 (DQ396010) & 97 \\
\hline EPR3968-O8a-Bc72 & alpha-Proteobacteria & Uncultured Hyphomicrobium sp. clone JdFBGBact_73 (DQ070833) & 99 \\
\hline EPR3968-O8a-Bc73 & CFB group & Uncultured marine bacterium clone PB1.23 (DQ071072) & 95 \\
\hline EPR3968-O8a-Bc74 & alpha-Proteobacteria & Uncultured bacterium clone G74 (DQ480486) & 95 \\
\hline EPR3968-O8a-Bc75 & alpha-Proteobacteria & Uncultured alpha proteobacterium clone MBAE27 (AJ567586) & 99 \\
\hline EPR3968-O8a-Bc76 & alpha-Proteobacteria & Uncultured bacterium clone: SRRB46 (AB240517) & 92 \\
\hline EPR3968-O8a-Bc77 & alpha-Proteobacteria & Uncultured alpha proteobacterium clone LiUU-9-26 (AY509403) & 90 \\
\hline EPR3968-O8a-Bc78 & gamma-Proteobacteria & Uncultured gamma proteobacterium MERTZ_0CM_58 ( & 99 \\
\hline EPR3968-O8a-Bc79 & gamma-Proteobacteria & Uncultured bacterium clone LC1133B-23 (DQ270615) & 93 \\
\hline EPR3968-O8a-Bc80 & Planctomycetes & planctomycete str. 292 (AF424134) & 94 \\
\hline EPR3968-O8a-Bc81 & gamma-Proteobacteria & Uncultured bacterium clone DBBB-38 (AY226290) & 97 \\
\hline EPR3968-O8a-Bc82 & gamma-Proteobacteria & Uncultured bacterium clone FS142-64B-02 (DQ513020) & 96 \\
\hline EPR3968-O8a-Bc83 & gamma-Proteobacteria & Uncultured bacterium clone SZB76 (AM176844) & 97 \\
\hline EPR3968-O8a-Bc84 & Actinobacteria & Uncultured organism clone ctg_CGOAA22 (DQ39550) & 98 \\
\hline EPR3968-O8a-Bc86 & Planctomycetes & Uncultured planctomycete clone LC1-32 (DQ289903) & 91 \\
\hline EPR3968-O8a-Bc87 & alpha-Proteobacteria & Uncultured alpha proteobacterium clone:JTB359 (AB015247) & 97 \\
\hline EPR3968-O8a-Bc88 & Unidentified & Unidentified bacterium clone: BD7-2 (AB015578) & 98 \\
\hline EPR3968-O8a-Bc89 & alpha-Proteobacteria & Uncultured alpha proteobacterium clone:JTB359 (AB015247) & 98 \\
\hline EPR3968-O8a-Bc90 & CFB group & Uncultured bacterium clone ELB16-214 (DQ015814) & 90 \\
\hline EPR3968-O8a-Bc91 & gamma-Proteobacteria & Legionella sp. OA32 (AB058916) & 96 \\
\hline EPR3968-O8a-Bc92 & alpha-Proteobacteria & Uncultured bacterium clone Napoli-1B-13 (AY592570) & 98 \\
\hline EPR3970-MO1A-Bc2 & alpha-Proteobacteria & $\begin{array}{l}\text { Uncultured alpha proteobacterium clone Belgica2005/10-140-4 } \\
\text { (DQ351777) }\end{array}$ & 99 \\
\hline EPR3970-MO1A-Bc3 & alpha-Proteobacteria & Uncultured alpha proteobacterium clone JdFBGBact_40 (DQ070827) & 97 \\
\hline EPR3970-MO1A-Bc4 & CFB group & Uncultured organism clone ctg_BRRAA93 (DQ395351) & 99 \\
\hline EPR3970-MO1A-Bc5 & alpha-Proteobacteria & Uncultured alpha proteobacterium clone LC1-25 (DQ289899) & 94 \\
\hline EPR3970-MO1A-Bc6 & gamma-Proteobacteria & Uncultured gamma proteobacterium clone:JTB255 (AB015254) & 98 \\
\hline EPR3970-MO1A-Bc8 & Unidentified & Unidentified bacterium clone: BD7-2 (AB015578) & 98 \\
\hline EPR3970-MO1A-Bc9 & Planctomycetes & Uncultured bacterium clone JG35+U1-AG121 (AM114433) & 94 \\
\hline EPR3970-MO1A-Bc10 & gamma-Proteobacteria & $\begin{array}{l}\text { Uncultured gamma proteobacterium clone Belgica2005/10-140-23 } \\
\text { (DQ351795) }\end{array}$ & 97 \\
\hline EPR3970-MO1A-Bc11 & alpha-Proteobacteria & Uncultured alpha proteobacterium clone JdFBGBact_40 (DQ070827) & 98 \\
\hline EPR3970-MO1A-Bc13 & gamma-Proteobacteria & $\begin{array}{l}\text { Uncultured gamma proteobacterium clone Belgica2005/10-140-5 } \\
\text { (DQ351778) }\end{array}$ & 99 \\
\hline EPR3970-MO1A-Bc14 & CFB group & Uncultured Cytophaga sp. clone JdFBGBact_34 (DQ070826) & 100 \\
\hline EPR3970-MO1A-Bc15 & gamma-Proteobacteria & Uncultured gamma proteobacterium clone E01-9C-26 (AJ581351) & 91 \\
\hline EPR3970-MO1A-Bc16 & gamma-Proteobacteria & Ifremeria nautilei gill symbiont (AB189713) & 93 \\
\hline EPR3970-MO1A-Bc17 & Actinobacteria & Uncultured bacterium clone: IheB3-34 (AB099989) & 93 \\
\hline EPR3970-MO1A-Bc18 & alpha-Proteobacteria & Uncultured alpha proteobacterium clone JdFBGBact_40 (DQ070827) & 97 \\
\hline EPR3970-MO1A-Bc19 & alpha-Proteobacteria & Uncultured organism clone ctg_CGOAB55 (DQ395481) & 92 \\
\hline EPR3970-MO1A-Bc20 & delta-Proteobacteria & Uncultured delta proteobacterium clone MBMPE81 (AJ567571) & 97 \\
\hline EPR3970-MO1A-Bc21 & gamma-Proteobacteria & $\begin{array}{l}\text { Uncultured gamma proteobacterium clone Belgica2005/10-140-5 } \\
\text { (DQ351778) }\end{array}$ & 99 \\
\hline EPR3970-MO1A-Bc23 & alpha-Proteobacteria & Uncultured organism clone ctg_CGOAB13 (DQ395499) & 95 \\
\hline EPR3970-MO1A-Bc24 & gamma-Proteobacteria & $\begin{array}{l}\text { Uncultured gamma proteobacterium clone Belgica2005/10-ZG-6 } \\
\text { (DQ351802) }\end{array}$ & 94 \\
\hline EPR3970-MO1A-Bc25 & alpha-Proteobacteria & Uncultured Hyphomicrobium clone JdFBGBact_73 (DQ070833) & 99 \\
\hline EPR3970-MO1A-Bc26 & Planctomycetes & Uncultured planctomycete clone LD1-PA40 (AY114326) & 97 \\
\hline EPR3970-MO1A-Bc27 & Nitrospirae & $\begin{array}{l}\text { Uncultured Nitrospiraeles bacterium clone Belgica2005/10-ZG-15 } \\
\text { (DQ351808) }\end{array}$ & 99 \\
\hline EPR3970-MO1A-Bc28 & alpha-Proteobacteria & Uncultured organism clone ctg CGOAB13 (DQ395499) & 95 \\
\hline EPR3970-MO1A-Bc29 & gamma-Proteobacteria & $\begin{array}{l}\text { Uncultured gamma proteobacterium clone Belgica2005/10-140-23 } \\
\text { (DQ351795) }\end{array}$ & 97 \\
\hline EPR3970-MO1A-Bc30 & Actinobacteria & Uncultured actinobacterium clone AT-s2-33 (AY225655) & 96 \\
\hline
\end{tabular}




\begin{tabular}{|c|c|c|c|}
\hline Clone & $\begin{array}{l}\text { Phylogenetic } \\
\text { Affiliation }\end{array}$ & Closest 16S rRNA BLAST match (accession number) & $\begin{array}{c}\text { Identity } \\
(\%)\end{array}$ \\
\hline EPR3970-MO1A-Bc31 & Acidobacteria & Unidentified bacterium wb1_A08 (AF317741) & 93 \\
\hline EPR3970-MO1A-Bc32 & gamma-Proteobacteria & Uncultured gamma proteobacterium clone AT-s80 (AY225635) & 99 \\
\hline EPR3970-MO1A-Bc33 & Actinobacteria & Uncultured Gram-positive bacterium clone JdFBGBact_23 (DQ07082: & 99 \\
\hline EPR3970-MO1A-Bc34 & gamma-Proteobacteria & Uncultured bacterium clone B185 (AY375060) & 99 \\
\hline EPR3970-MO1A-Bc35 & Planctomycetes & Uncultured Pirellula sp. clone 9NBGBact_17 (DQ070794) & 92 \\
\hline EPR3970-MO1A-Bc36 & gamma-Proteobacteria & $\begin{array}{l}\text { Uncultured gamma proteobacterium clone Belgica2005/10-ZG-21 } \\
\text { (DQ351813) }\end{array}$ & 96 \\
\hline EPR3970-MO1A-Bc37 & gamma-Proteobacteria & Uncultured bacterium clone B185 (AY375060) & 99 \\
\hline EPR3970-MO1A-Bc38 & gamma-Proteobacteria & Alcanivorax sp. I4 (AB053125) & 90 \\
\hline EPR3970-MO1A-Bc39 & Unidentified & Uncultured Verrucomicrobia bacterium clone Sylt 22 (AM040118) & 92 \\
\hline EPR3970-MO1A-Bc41 & gamma-Proteobacteria & Uncultured gamma proteobacterium clone MBMPE27 (AJ567545) & 97 \\
\hline EPR3970-MO1A-Bc42 & alpha-Proteobacteria & Uncultured organism clone ctg_CGOAB55 (DQ395481) & 92 \\
\hline EPR3970-MO1A-Bc43 & Planctomycetes & Uncultured planctomycete clone HPDOMS1G05 (AY852002) & 98 \\
\hline EPR3970-MO1A-Bc44 & Planctomycetes & Uncultured bacterium MERTZ_21CM_74 (AF424485) & 97 \\
\hline EPR3970-MO1A-Bc45 & Unidentified & Uncultured actinobacterium clone AT-s3-3 (AY225656) & 93 \\
\hline EPR3970-MO1A-Bc46 & Unidentified & Uncultured organism clone ctg_CGOCA20 (DQ395515) & 99 \\
\hline EPR3970-MO1A-Bc47 & gamma-Proteobacteria & $\begin{array}{l}\text { Uncultured gamma proteobacterium clone Belgica2005/10-ZG-6 } \\
\text { (DQ351802) }\end{array}$ & 97 \\
\hline EPR3970-MO1A-Bc48 & delta-Proteobacteria & Uncultured delta proteobacterium clone:JTB38 (AB015243) & 97 \\
\hline EPR3970-MO1A-Bc49 & alpha-Proteobacteria & Uncultured Hyphomicrobium sp. clone JdFBGBact_73 (DQ070833) & 99 \\
\hline EPR3970-MO1A-Bc50 & Unidentified & Uncultured organism clone ctg_BRRAA88 (DQ395368) & 100 \\
\hline EPR3970-MO1A-Bc51 & Actinobacteria & Unidentified bacterium wb1_P06 (AF317769) & 98 \\
\hline EPR3970-MO1A-Bc52 & Gemmatimonadetes & Uncultured bacterium clone KS77 (AF328213) & 95 \\
\hline EPR3970-MO1A-Bc53 & alpha-Proteobacteria & Uncultured bacterium clone Napoli-1B-13 (AY592570) & 98 \\
\hline EPR3970-MO1A-Bc54 & gamma-Proteobacteria & Uncultured gamma proteobacterium clone AT-s80 (AY225635) & 99 \\
\hline EPR3970-MO1A-Bc55 & Actinobacteria & Uncultured bacterium clone AKIW984 (DQ129354) & 95 \\
\hline EPR3970-MO1A-Bc56 & gamma-Proteobacteria & Pseudomonas sp. 3A_6 (AY689025) & 90 \\
\hline EPR3970-MO1A-Bc57 & gamma-Proteobacteria & Uncultured bacterium clone B185 (AY375060) & 98 \\
\hline EPR3970-MO1A-Bc58 & Actinobacteria & Uncultured bacterium clone FS266-92B-03 (DQ513090) & 95 \\
\hline EPR3970-MO1A-Bc59 & Planctomycetes & Uncultured planctomycete MERTZ_21CM_26(AF424480) & 96 \\
\hline EPR3970-MO1A-Bc60 & alpha-Proteobacteria & Uncultured soil bacterium clone M43_Pitesti (DQ378260) & 92 \\
\hline EPR3970-MO1A-Bc61 & Planctomycetes & Uncultured planctomycete clone Sylt 12 (AM040108) & 94 \\
\hline EPR3970-MO1A-Bc63 & Acidobacteria & Uncultured Acidobacteria bacterium clone CAL7 (DQ139449) & 92 \\
\hline EPR3970-MO1A-Bc64 & Actinobacteria & Uncultured bacterium partial (AJ966584) & 99 \\
\hline EPR3970-MO1A-Bc65 & Unidentified & Uncultured bacterium clone CV90 (DQ499320) & 89 \\
\hline EPR3970-MO1A-Bc66 & Unidentified & Uncultured organism clone ctg_CGOCA20 (DQ395515) & 99 \\
\hline EPR3970-MO1A-Bc67 & alpha-Proteobacteria & Uncultured Hyphomicrobium sp. clone JdFBGBact_73 (DQ070833) & 99 \\
\hline EPR3970-MO1A-Bc68 & Unidentified & Uncultured organism clone ctg_BRRAA88 (DQ395368) & 100 \\
\hline EPR3970-MO1A-Bc69 & alpha-Proteobacteria & Uncultured organism clone ctg_CGOAB13 (DQ395499) & 95 \\
\hline $\begin{array}{l}\text { EPR3970-MO1A-Bc70 } \\
\text { EPR3970-MO1A-Bc71 }\end{array}$ & $\begin{array}{l}\text { Actinobacteria } \\
\text { Acidobacteria }\end{array}$ & $\begin{array}{l}\text { Uncultured bacterium clone E17 (AJ966591) } \\
\text { uncultured Holophaga/Acidobacterium Sva0515 (AJ241004) }\end{array}$ & $\begin{array}{l}98 \\
97\end{array}$ \\
\hline EPR3970-MO1A-Bc73 & alpha-Proteobacteria & Uncultured bacterium gene clone: IheB2-3 (AB099936) & 95 \\
\hline EPR3970-MO1A-Bc74 & Acidobacteria & Uncultured soil bacterium clone M49_Pitesti (DQ378265) & 94 \\
\hline EPR3970-MO1A-Bc75 & Actinobacteria & Uncultured bacterium clone E19 (AJ966592) & 95 \\
\hline EPR3970-MO1A-Bc76 & gamma-Proteobacteria & Uncultured bacterium clone Napoli-1B-61 (AY592616) & 96 \\
\hline EPR3970-MO1A-Bc77 & Planctomycetes & Uncultured Pirellula sp. clone 9NBGBact_17 (DQ070794) & 93 \\
\hline EPR3970-MO1A-Bc78 & Planctomycetes & Uncultured bacterium clone aab55f08 (DQ814931) & 95 \\
\hline EPR3970-MO1A-Bc79 & Acidobacteria & Uncultured Acidobacteriaceae bacterium clone AT-s3-59 (AY225642) & 95 \\
\hline EPR3970-MO1A-Bc80 & gamma-Proteobacteria & Uncultured gamma proteobacterium clone AT-s80 (AY225635) & 99 \\
\hline EPR3970-MO1A-Bc81 & gamma-Proteobacteria & $\begin{array}{l}\text { Uncultured gamma proteobacterium clone Belgica2005/10-120-9 } \\
\text { (DQ351744) }\end{array}$ & 98 \\
\hline EPR3970-MO1A-Bc82 & delta-Proteobacteria & Unidentified bacterium wb1_A15 (AF317744) & 93 \\
\hline EPR3970-MO1A-Bc83 & gamma-Proteobacteria & Uncultured bacterium clone B185 (AY375060) & 99 \\
\hline
\end{tabular}




\begin{tabular}{|c|c|c|c|}
\hline Clone & $\begin{array}{l}\text { Phylogenetic } \\
\text { Affiliation }\end{array}$ & Closest 16S rRNA BLAST match (accession number) & $\begin{array}{c}\text { Identity } \\
\text { (\%) }\end{array}$ \\
\hline EPR3970-MO1A-Bc84 & gamma-Proteobacteria & $\begin{array}{l}\text { Uncultured gamma proteobacterium clone Belgica2005/10-ZG-6 } \\
\text { (DQ351802) }\end{array}$ & 94 \\
\hline EPR3970-MO1A-Bc85 & gamma-Proteobacteria & Uncultured gamma proteobacterium clone MBMPE27 (AJ567545) & 97 \\
\hline EPR3970-MO1A-Bc86 & Unidentified & Uncultured Verrucomicrobia bacterium clone LD1-PA34 (AY114322) & 95 \\
\hline EPR3970-MO1A-Bc87 & alpha-Proteobacteria & Uncultured bacterium clone A56 (AY373412) & 95 \\
\hline EPR3970-MO1A-Bc88 & Unidentified & Uncultured organism clone ctg_CGOCA20 (DQ395515) & 99 \\
\hline EPR3970-MO1A-Bc89 & Unidentified & Uncultured organism clone ctg_CGOCA20 (DQ395515) & 99 \\
\hline EPR3970-MO1A-Bc90 & gamma-Proteobacteria & Uncultured gamma proteobacterium clone:JTB255 (AB015254) & 98 \\
\hline EPR4059-B2-Bc1 & alpha-Proteobacteria & Uncultured bacteriumclone SZB47 (AM176849) & 97 \\
\hline EPR4059-B2-Bc3 & gamma-Proteobacteria & Uncultured bacterium clone DBBB-38 (AY226290) & 96 \\
\hline EPR4059-B2-Bc5 & alpha-Proteobacteria & Amaricoccus tamworthensis (U88044) & 95 \\
\hline EPR4059-B2-Bc6 & Actinobacteria & Uncultured organism clone ctg_CGOAA79 (DQ395467) & 97 \\
\hline EPR4059-B2-Bc7 & alpha-Proteobacteria & Uncultured organism clone ctg_CGOAB13 (DQ395499) & 95 \\
\hline EPR4059-B2-Bc8 & alpha-Proteobacteria & Uncultured alpha proteobacterium clone LC1-34 (DQ289904) & 92 \\
\hline EPR4059-B2-Bc9 & alpha-Proteobacteria & Uncultured organism clone ctg_CGOAA09 (DQ395453) & 99 \\
\hline EPR4059-B2-Bc10 & alpha-Proteobacteria & Uncultured bacterium gene clone: IheB2-3 (AB099936) & 99 \\
\hline EPR4059-B2-Bc11 & gamma-Proteobacteria & Dechloromarinus chlorophilus (AF170359) & 90 \\
\hline EPR4059-B2-Bc12 & Unidentified & Uncultured organism clone ctg_BRRAA88 (DQ395368) & 98 \\
\hline EPR4059-B2-Bc13 & alpha-Proteobacteria & Uncultured bacterium clone Napoli-1B-13 (AY592570) & 99 \\
\hline EPR4059-B2-Bc14 & gamma-Proteobacteria & Uncultured bacterium clone DBBB-38 (AY226290) & 96 \\
\hline EPR4059-B2-Bc15 & alpha-Proteobacteria & Uncultured alpha proteobacterium clone LC1-34 (DQ289904) & 93 \\
\hline EPR4059-B2-Bc16 & alpha-Proteobacteria & Uncultured Hyphomicrobium sp. clone JdFBGBact_73 (DQ070833) & 99 \\
\hline EPR4059-B2-Bc17 & alpha-Proteobacteria & Rhodospirillaceae bacterium CL-UU02 (DQ401091) & 96 \\
\hline EPR4059-B2-Bc18 & gamma-Proteobacteria & $\begin{array}{l}\text { Uncultured gamma proteobacterium clone Belgica2005/10-130-9 } \\
\text { (DQ351756) }\end{array}$ & 99 \\
\hline EPR4059-B2-Bc20 & Unidentified & Uncultured organism clone ctg_CGOCA20 (DQ395515) & 100 \\
\hline EPR4059-B2-Bc21 & alpha-Proteobacteria & Uncultured alpha proteobacterium clone LC1-34 (DQ289904) & 93 \\
\hline EPR4059-B2-Bc22 & gamma-Proteobacteria & Uncultured bacterium clone LC1133B-61 (DQ270619) & 92 \\
\hline EPR4059-B2-Bc23 & Planctomycetes & Uncultured planctomycete clone SC3-8 (DQ289943) & 97 \\
\hline EPR4059-B2-Bc24 & Planctomycetes & Uncultured planctomycete clone SC3-8 (DQ289943) & 96 \\
\hline EPR4059-B2-Bc27 & alpha-Proteobacteria & Uncultured Hyphomicrobium sp. clone JdFBGBact_73 (DQ070833) & 99 \\
\hline EPR4059-B2-Bc30 & Planctomycetes & Uncultured planctomycete clone AKYG983 (AY922083) & 98 \\
\hline EPR4059-B2-Bc31 & alpha-Proteobacteria & Mucus bacterium 23 (AY654769) & 95 \\
\hline EPR4059-B2-Bc34 & alpha-Proteobacteria & Uncultured bacterium clone SZB47 (AM176849) & 97 \\
\hline EPR4059-B2-Bc35 & gamma-Proteobacteria & Dechloromarinus chlorophilus (AF170359) & 90 \\
\hline EPR4059-B2-Bc36 & gamma-Proteobacteria & Pseudomonas sp. RLD-1 (DQ339153) & 90 \\
\hline EPR4059-B2-Bc37 & CFB group & Psychroserpens sp. 18III/A01/061 (AY576714) & 94 \\
\hline EPR4059-B2-Bc38 & alpha-Proteobacteria & Uncultured bacterium clone G74 (DQ480486) & 95 \\
\hline EPR4059-B2-Bc39 & gamma-Proteobacteria & Uncultured gamma proteobacterium clone MBMPE52 (AJ567564) & 95 \\
\hline EPR4059-B2-Bc40 & Actinobacteria & Uncultured bacterium clone A20 (AY373407) & 99 \\
\hline EPR4059-B2-Bc41 & Unidentified & Uncultured organism clone ctg_CGOCA20 (DQ395515) & 94 \\
\hline EPR4059-B2-Bc44 & alpha-Proteobacteria & Uncultured alpha proteobacterium clone LC1-34 (DQ289904) & 92 \\
\hline EPR4059-B2-Bc46 & Planctomycetes & Uncultured planctomycete clone SC3-8 (DQ289943) & 96 \\
\hline EPR4059-B2-Bc47 & Acidobacteria & Uncultured sponge symbiont PAUC26f (AF186410) & 92 \\
\hline EPR4059-B2-Bc49 & alpha-Proteobacteria & Uncultured organism clone ctg_CGOAB55 (DQ395481) & 92 \\
\hline EPR4059-B2-Bc51 & gamma-Proteobacteria & $\begin{array}{l}\text { Uncultured gamma proteobacterium clone Belgica2005/10-140-23 } \\
\text { (DQ351795) }\end{array}$ & 98 \\
\hline EPR4059-B2-Bc52 & Acidobacteria & Uncultured bacterium clone FS266-75B-03 (DQ513088) & 93 \\
\hline EPR4059-B2-Bc53 & gamma-Proteobacteria & $\begin{array}{l}\text { Uncultured gamma proteobacterium clone Belgica2005/10-140-23 } \\
\text { (DQ351795) }\end{array}$ & 98 \\
\hline EPR4059-B2-Bc54 & Planctomycetes & Uncultured bacterium clone 031H09_P_BA_P3 (BX294860) & 95 \\
\hline EPR4059-B2-Bc55 & alpha-Proteobacteria & Uncultured alpha proteobacterium clone:JTB131 (AB015245) & 98 \\
\hline EPR4059-B2-Bc57 & gamma-Proteobacteria & Uncultured gamma proteobacterium clone AT-s68 (AY225627) & 98 \\
\hline
\end{tabular}




\begin{tabular}{|c|c|c|c|}
\hline Clone & $\begin{array}{l}\text { Phylogenetic } \\
\text { Affiliation }\end{array}$ & Closest 16S rRNA BLAST match (accession number) & $\begin{array}{c}\text { Identity } \\
\text { (\%) }\end{array}$ \\
\hline EPR4059-B2-Bc58 & gamma-Proteobacteria & Uncultured bacterium clone P101-46 (DQ009038) & 94 \\
\hline EPR4059-B2-Bc59 & alpha-Proteobacteria & Uncultured bacterium clone: RB312 (AB240333) & 97 \\
\hline EPR4059-B2-Bc60 & alpha-Proteobacteria & Uncultured organism clone ctg_CGOAA09 (DQ395453) & 99 \\
\hline EPR4059-B2-Bc61 & alpha-Proteobacteria & $\begin{array}{l}\text { Uncultured proteobacterium clone DR938CH110701SACH95 } \\
\text { (DQ230971) }\end{array}$ & 90 \\
\hline EPR4059-B2-Bc64 & alpha-Proteobacteria & Uncultured bacterium clone Napoli-1B-13 (AY592570) & 98 \\
\hline EPR4059-B2-Bc65 & Planctomycetes & Uncultured bacterium clone FS275-22B-03 (DQ513104) & 92 \\
\hline EPR4059-B2-Bc66 & Chlamydiae & Simkania negevensis (U68460) & 89 \\
\hline EPR4059-B2-Bc67 & gamma-Proteobacteria & Uncultured gamma proteobacterium clone PI_4h10g (AY580822) & 98 \\
\hline EPR4059-B2-Bc68 & alpha-Proteobacteria & Uncultured Hyphomicrobium sp. clone JdFBGBact_73 (DQ070833) & 99 \\
\hline EPR4059-B2-Bc69 & Actinobacteria & Uncultured organism clone ctg_CGOAA79 (DQ395467) & 93 \\
\hline EPR4059-B2-Bc70 & alpha-Proteobacteria & Mucus bacterium 23 (AY654769) & 95 \\
\hline EPR4059-B2-Bc71 & alpha-Proteobacteria & Uncultured bacterium clone: IheB2-3 (AB099936) & 99 \\
\hline EPR4059-B2-Bc72 & CFB group & Psychroserpens sp. 18III/A01/061 (AY576714) & 95 \\
\hline EPR4059-B2-Bc73 & Actinobacteria & Uncultured organism clone ctg_CGOAA79 (DQ395467) & 98 \\
\hline EPR4059-B2-Bc74 & Actinobacteria & Unidentified bacterium wb1_P06 (AF317769) & 99 \\
\hline EPR4059-B2-Bc75 & gamma-Proteobacteria & Dechloromarinus chlorophilus (AF170359) & 90 \\
\hline EPR4059-B2-Bc76 & alpha-Proteobacteria & Uncultured organism clone ctg_CGOAB13 (DQ395499) & 100 \\
\hline EPR4059-B2-Bc77 & alpha-Proteobacteria & Uncultured Hyphomicrobium sp. clone JdFBGBact_73 (DQ070833) & 99 \\
\hline EPR4059-B2-Bc78 & Acidobacteria & Uncultured bacterium clone NS71 (AY172653) & 94 \\
\hline EPR4059-B2-Bc81 & alpha-Proteobacteria & Uncultured bacterium clone G74 (DQ480486) & 95 \\
\hline EPR4059-B2-Bc82 & gamma-Proteobacteria & Oceanospirillum sp. MED92 (AY136116) & 87 \\
\hline EPR4059-B2-Bc83 & Actinobacteria & Unidentified bacterium wb1_P06 (AF317769) & 99 \\
\hline EPR4059-B2-Bc84 & alpha-Proteobacteria & Uncultured bacterium clone SZB47 (AM176849) & 98 \\
\hline EPR4059-B2-Bc85 & Planctomycetes & Uncultured planctomycete clone GWS-K12 (AY515488) & 98 \\
\hline EPR4059-B2-Bc86 & alpha-Proteobacteria & Uncultured bacterium clone SZB47 (AM176849) & 97 \\
\hline EPR4059-B2-Bc87 & alpha-Proteobacteria & Uncultured bacterium clone SZB47 (AM176849) & 98 \\
\hline EPR4059-B2-Bc88 & gamma-Proteobacteria & Dechloromarinus chlorophilus (AF170359) & 90 \\
\hline EPR4059-B2-Bc89 & alpha-Proteobacteria & Uncultured organism clone ctg_CGOAB55 (DQ395481) & 99 \\
\hline EPR4059-B2-Bc90 & delta-Proteobacteria & Uncultured delta proteobacterium clone LC1-13 (DQ289938) & 91 \\
\hline EPR4059-B2-Bc91 & alpha-Proteobacteria & Uncultured alpha proteobacterium clone:JTB131 (AB015245) & 98 \\
\hline EPR4059-B2-Bc92 & alpha-Proteobacteria & Uncultured organism clone ctg_CGOAA08 (DQ395424) & 98 \\
\hline EPR4059-B2-Bc93 & Unidentified & Uncultured organism clone ctg_CGOCA20 (DQ395515) & 94 \\
\hline EPR4055-N3-Bc10 & gamma-Proteobacteria & Marinobacter sp. ASs2019 (DQ665806) & 100 \\
\hline EPR4055-N3-Bc11 & gamma-Proteobacteria & Uncultured Colwellia clone: JT58-11 (AB189338) & 99 \\
\hline EPR4055-N3-Bc12 & gamma-Proteobacteria & Marinobacter sp. ASs2019 (DQ665806) & 99 \\
\hline EPR4055-N3-Bc13 & gamma-Proteobacteria & Uncultured Colwellia clone: JT58-11 (AB189338) & 98 \\
\hline EPR4055-N3-Bc14 & alpha-Proteobacteria & Uncultured organism clone ctg_CGOF269 (DQ395675) & 99 \\
\hline EPR4055-N3-Bc15 & gamma-Proteobacteria & Colwellia sp. MT41 (DQ027051) & 95 \\
\hline EPR4055-N3-Bc17 & gamma-Proteobacteria & Uncultured Colwellia clone: JT58-11 (AB189338) & 99 \\
\hline EPR4055-N3-Bc18 & gamma-Proteobacteria & Marinobacter sp. ASs2019 (DQ665806) & 100 \\
\hline EPR4055-N3-Bc19 & gamma-Proteobacteria & Uncultured bacterium clone F2 (AY375114) & 98 \\
\hline EPR4055-N3-Bc20 & gamma-Proteobacteria & Moritella marina (AB121097) & 99 \\
\hline EPR4055-N3-Bc21 & gamma-Proteobacteria & Marinobacter sp. ASs2019 (DQ665806) & 100 \\
\hline EPR4055-N3-Bc22 & gamma-Proteobacteria & Uncultured Pseudomonas sp. clone SQ2a_Pitesti (DQ366084) & 99 \\
\hline EPR4055-N3-Bc23 & gamma-Proteobacteria & Uncultured Pseudomonas sp. clone SQ2a_Pitesti (DQ366084) & 100 \\
\hline EPR4055-N3-Bc25 & gamma-Proteobacteria & Halomonas sp. MAN K30 (AB166936) & 100 \\
\hline EPR4055-N3-Bc26 & gamma-Proteobacteria & Moritella marina (AB121097) & 99 \\
\hline EPR4055-N3-Bc27 & gamma-Proteobacteria & Moritella marina (AB121097) & 99 \\
\hline $\begin{array}{l}\text { EPR4055-N3-Bc28 } \\
\text { EPR4055-N3-Bc29 }\end{array}$ & $\begin{array}{l}\text { gamma-Proteobacteria } \\
\text { gamma-Proteobacteria }\end{array}$ & $\begin{array}{l}\text { Marinobacter sp. ASs2019 (DQ665806) } \\
\text { Marinobacter sp. ASs2019 (DQ665806) }\end{array}$ & $\begin{array}{l}99 \\
99\end{array}$ \\
\hline EPR4055-N3-Bc30 & gamma-Proteobacteria & Uncultured organism clone ctg_NISA124 (DQ396363) & 99 \\
\hline
\end{tabular}




\begin{tabular}{|c|c|c|c|}
\hline Clone & $\begin{array}{l}\text { Phylogenetic } \\
\text { Affiliation }\end{array}$ & Closest 16S rRNA BLAST match (accession number) & $\begin{array}{l}\text { Identity } \\
(\%)\end{array}$ \\
\hline EPR4055-N3-Bc31 & gamma-Proteobacteria & Marinobacter sp. ASs2019 (DQ665806) & 99 \\
\hline EPR4055-N3-Bc35 & alpha-Proteobacteria & Uncultured bacterium clone CM9 (DQ832646) & 97 \\
\hline EPR4055-N3-Bc38 & beta-Proteobacteria & Curvibacter gracilis (AB109889) & 100 \\
\hline EPR4055-N3-Bc39 & gamma-Proteobacteria & Marinobacter sp. ASs2019 (DQ665806) & 99 \\
\hline EPR4055-N3-Bc42 & gamma-Proteobacteria & Uncultured bacterium clone D12 (AY375136) & 99 \\
\hline EPR4055-N3-Bc45 & gamma-Proteobacteria & Uncultured Colwellia clone: JT58-11 (AB189338) & 98 \\
\hline EPR4055-N3-Bc46 & gamma-Proteobacteria & Uncultured Colwellia clone: JT58-11 (AB189338) & 98 \\
\hline EPR4055-N3-Bc47 & gamma-Proteobacteria & Marinobacter sp. ASs2019 (DQ665806) & 99 \\
\hline EPR4055-N3-Bc50 & gamma-Proteobacteria & Alteromonas sp. U70 (AJ832999) & 99 \\
\hline EPR4055-N3-Bc51 & gamma-Proteobacteria & Marinobacter sp. ASs2019 (DQ665806) & 99 \\
\hline EPR4055-N3-Bc52 & gamma-Proteobacteria & Halomonas sp. 3022 (AM110977) & 100 \\
\hline EPR4055-N3-Bc53 & gamma-Proteobacteria & Uncultured Colwellia clone: JT58-11 (AB189338) & 99 \\
\hline EPR4055-N3-Bc54 & gamma-Proteobacteria & Uncultured bacterium clone F2 (AY375114) & 98 \\
\hline EPR4055-N3-Bc55 & gamma-Proteobacteria & Uncultured Arctic sea ice bacterium clone ARKXV/1-140 (AY165592 & 100 \\
\hline EPR4055-N3-Bc59 & gamma-Proteobacteria & Marine bacterium SCRIPPS_740 (AF359550) & 100 \\
\hline EPR4055-N3-Bc60 & alpha-Proteobacteria & Uncultured organism clone ctg_CGOF083 (DQ395914) & 100 \\
\hline EPR4055-N3-Bc61 & gamma-Proteobacteria & Uncultured Colwellia clone: JT58-11 (AB189338) & 98 \\
\hline EPR4055-N3-Bc62 & gamma-Proteobacteria & Uncultured Colwellia clone: JT58-11 (AB189338) & 98 \\
\hline EPR4055-N3-Bc63 & gamma-Proteobacteria & Uncultured Colwellia clone: JT58-11 (AB189338) & 98 \\
\hline EPR4055-N3-Bc67 & gamma-Proteobacteria & Uncultured Colwellia clone: JT58-11 (AB189338) & 99 \\
\hline EPR4055-N3-Bc70 & gamma-Proteobacteria & Halomonas sp. 3022 (AM110977) & 99 \\
\hline EPR4055-N3-Bc71 & alpha-Proteobacteria & Sphingomonas sp. oral clone AV069 (AF385529) & 100 \\
\hline EPR4055-N3-Bc73 & gamma-Proteobacteria & Uncultured Colwellia clone: JT58-11 (AB189338) & 99 \\
\hline EPR4055-N3-Bc74 & gamma-Proteobacteria & Halomonas frigidi (AJ431369) & 100 \\
\hline EPR4055-N3-Bc75 & gamma-Proteobacteria & Uncultured Colwellia clone: JT58-11 (AB189338) & 98 \\
\hline EPR4055-N3-Bc76 & gamma-Proteobacteria & Moritella marina (AB121097) & 99 \\
\hline EPR4055-N3-Bc77 & gamma-Proteobacteria & Uncultured Pseudomonas sp. clone SQ2a_Pitesti (DQ366084) & 100 \\
\hline EPR4055-N3-Bc78 & gamma-Proteobacteria & Halomonas sp. 3022 (AM110977) & 100 \\
\hline EPR4055-N3-Bc79 & gamma-Proteobacteria & Uncultured Colwellia clone: JT58-11 (AB189338) & 99 \\
\hline EPR4055-N3-Bc80 & gamma-Proteobacteria & Marinobacter sp. ASs2019 (DQ665806) & 99 \\
\hline EPR4055-N3-Bc81 & gamma-Proteobacteria & Uncultured Colwellia clone: JT58-11 (AB189338) & 99 \\
\hline EPR4055-N3-Bc82 & gamma-Proteobacteria & Uncultured Colwellia clone: JT58-11 (AB189338) & 98 \\
\hline EPR4055-N3-Bc83 & gamma-Proteobacteria & Uncultured Colwellia clone: JT58-11 (AB189338) & 98 \\
\hline EPR4055-N3-Bc84 & gamma-Proteobacteria & Uncultured bacterium clone CTD005-53B-02 (DQ513057) & 100 \\
\hline EPR4055-N3-Bc85 & beta-Proteobacteria & Curvibacter gracilis (AB109889) & 100 \\
\hline EPR4055-N3-Bc86 & gamma-Proteobacteria & Uncultured Colwellia clone: JT58-11 (AB189338) & 99 \\
\hline EPR4055-N3-Bc87 & gamma-Proteobacteria & Halomonas sp. 3022 (AM110977) & 99 \\
\hline EPR4055-N3-Bc88 & gamma-Proteobacteria & Marinobacter sp. ASs2019 (DQ665806) & 100 \\
\hline EPR4055-N3-Bc89 & alpha-Proteobacteria & Uncultured organism clone ctg_CGOF269 (DQ395675) & 99 \\
\hline EPR4055-N3-Bc90 & gamma-Proteobacteria & Uncultured Pseudomonas sp. clone SQ2a_Pitesti (DQ366084) & 100 \\
\hline EPR4055-N3-Bc91 & gamma-Proteobacteria & Uncultured Colwellia clone: JT58-11 (AB189338) & 99 \\
\hline $\begin{array}{l}\text { EPR4055-N3-Bc92 } \\
\text { EPR4055-N3-Bc93 }\end{array}$ & $\begin{array}{l}\text { gamma-Proteobacteria } \\
\text { gamma-Proteobacteria }\end{array}$ & $\begin{array}{l}\text { Alcanivorax sp. I2 (AB053126) } \\
\text { Uncultured organism clone ctg NISA077 (DQ396347) }\end{array}$ & $\begin{array}{l}100 \\
100\end{array}$ \\
\hline
\end{tabular}




\subsection{References}

Alt, J.C. (1995) Subseafloor processes in mid-ocean ridge hydrothermal systems. In Seafloor hydrothermal systems: physical, chemical, biological, and geological interactions. Humphris, S.E., Zierneberg, R.A., Mullineaux, L.S., and Thomson, R.E. (eds). Washington, DC: American Geophysical Union, pp. 85-114.

Altschul, S.F., Madden, T.L., Schaffer, A.A., Zhang, J., Zhang, Z., Miller, W., and Lipman, D.J. (1997) Gapped BLAST and PSI-blast: a new generation of protein database search programs. Nucl. Acids Res. 25: 3389-3402.

Bach, W., and Edwards, K.J. (2003) Iron and sulfide oxidation within the basaltic ocean crust: Implications for chemolithoautotrophic microbial biomass production. Geochim. Cosmochim. Acta 67: 3871-3887.

Barber, R.T. (1968) Dissolved organic carbon from deep water resists microbial oxidation. Nature 220.

Bowman, J.P., and McCuaig, R.D. (2003) Biodiversity, community structural shifts, and biogeography of prokaryotes within Anarcttic Continental Shelf Sediment. Appl. Environ. Microbiol. 69: 2463-2483.

Carbotte, S., and Macdonald, K.C. (1992) East Pacific Rise 8 degrees-10 degrees 30'N; evolution of ridge segments and discontinuities from Sea Marc II and threedimensional magnetic studies. J. Geophys. Res.97: 6959-6982.

Chao, A. (1984) Nonparametric estimation of the number of classes in a population. Scand. J. Statist. 11: 265-270.

Chao, A., Shen, T.J., and Hwang, W.H. (2006) The applications of Laplace's boundarymode approximations to estimate species richness and shared species richness. Aust. N. Z. J. Stat. 48: 117-128.

Chao, A., Chazdon, R.L., Colwell, R.K., and Shen, T.J. (2005) A new statistical approach for assessing similarity of species composition with incidence and abundance data. Ecol. Lett. 8: 148-159.

Cole, J.R., Chai, B., Marsh, T.L., Farris, R.J., Wang, Q., Kulam, S.A. et al. (2003) The ribosomal database project (RDP-II): previewing a new autoaligner that allows regular updates and the new prokaryotic taxonomy. Nucl. Acids Res. 31: 442-443.

Cowen, J.P., Giovannoni, S.J., Kenig, F., Johnson, H.P., Butterfield, D., Rappe, M.S. et al. (2003) Fluids from aging ocean crust that support microbial life. Science 299: $120-123$.

Delgado, O., Quillaguaman, J., Bakhtiar, S., Mattiasson, B., Gessesse, A., and HattiKaul, R. (2006) Nesterenkonia aethiopica sp. nov., an alkaliphilic, moderate halophile isolated from an Ethiopian soda lake. Int. J. Syst. Evol. Microbiol. 56: 1229-1232.

Eberhard, C., Wirsen, C.O., and Jannasch, H.W. (1995) Oxidation of polymetal sulfides by chemolithoautotrophic bacteria from deep-sea hydrothermal vents. Geomicrobiology Journal 13: 145-164.

Edwards, K.J., Bach, W., and McCollom, T.M. (2005) Geomicrobiology in oceanography: microbe-mineral interactions at and below the seafloor. TRENDS in Microbiology 13. 
Edwards, K.J., Bond, P.L., Gihring, T.M., and Banfield, J.F. (2000) An archaeal ironoxidizing extreme acidophile important in acid mine drainage. Science 287: 17961799.

Edwards, K.J., Rogers, D.R., Wirsen, C.O., and McCollom, T.M. (2003) Isolation and characterization of novel psychrophilic, neutrophilic, Fe-oxidizing, chemolithoautotrophic alpha- and gamma-proteobacteria from the deep sea. Appl. Environ. Microbiol. 69: 2906-2913.

Edwards, K.J., Bach, W., McCollom, T.M., and Rogers, D.R. (2004) Neutrophilic ironoxidizing bacteria in the ocean: Their habitats, diversity, and roles in mineral deposition, rock alteration, and biomass production in the deep-sea. Geomicrobiology Journal 21: 393-404.

Fornari, D.J., Haymon, R.M., Perfit, M.R., Gregg, T.K.P., Edwards, M.H. (1998) Axial summit trough of the East Pacific Rise $9^{\circ}-10^{\circ} \mathrm{N}$ : Geological characteristics and evolution of the axial zone of fast spreading mid-ocean ridges. J. Geophys. Res. 103: 9827-9856.

Gulledge, J., Ahmad, A., Steudler, P.A., Pomerantz, W.J., and Cavanaugh, C.M. (2001) Family- and genus-level 16S rRNA-targeted oligonucleotide probes for ecological studies of methanotrophic bacteria. Appl. Environ. Microbiol. 67: 4726-4733.

Huber, J., Butterfield, D., and Baross, J. (2003) Bacterial diversity in a subseafloor habitat following a deep-sea volcanic eruption. FEMS Microbiol. Ecol. 43: 393409.

Huber, J.A., Johnson, H.P., Butterfield, D.A., and Baross, J.A. (2006) Microbial life in ridge flank crustal fluids. Appl. Environ. Microbiol. 8: 88-99.

Karner, M.B., DeLong, E.F., and Karl, D.M. (2001) Archaeal dominance in the mesopelagic zone of the Pacific Ocean. Nature 409: 507-510.

Khan, S.T., Horiba, Y., Yamamoto, M., and Hiraishi, A. (2002) Members of the family Comamonadaceae as primary poly (3-hydroxybutyrate-co-3-hydroxyvalerate)degrading denitrifiers in activated sludge as revealed by a polyphasic approach. Appl. Environ. Microbiol. 68: 3206-3214.

Klappenbach, J.L., Saxman, P.R., Cole, J.R., and Schmidt, T.M. (2001) rrndb: the ribosomal RNA operon copy number database. Nucl. Acids Res. 29: 181-184.

Knittel, K., Boetius, A., Lemke, A., Eilers, H., Lochte, K., Pfannkuche, O. et al. (2003) Activity, distribution, and diversity of sulfate reducers and other bacteria in sediments above gas hydrate (Cascadia Margin, Oregon). Geomicrobiology Journal 20: 269-294.

Kwon, K.K., Lee, H.S., Yang, S.H., and Kim, S.J. (2005) Kordiimonas gwangyangensis gen. nov., sp. nov., a marine bacterium isolated from marine sediments that forms a distinct phyletic lineage (Kordiimonadales ord. nov.) in the 'Alphaproteobacteria'. Int. J. Syst. Evol. Microbiol. 55: 2033-2037.

Ley, R.E., Harris, J., K., Wilcox, J., Spear, J.R., Miller, S.R., Bebout, B.M. et al. (2006) Unexpected diversity and complexity of the Guerrero Negro hypersaline microbial mat. Appl. Environ. Microbiol. 72: 3685-3695.

Li, L., Kato, C., and Horikoshi, K. (1999) Microbial diversity in sediments collected from the deepest cold-seep area, the Japan Trench. Mar. Biotechnol. 1: 391-400. 
Lin, L.H., Hall, J., Onstott, T.C., Gihring, T.M., Sherwood Lollar, B., Boice, E. et al. (2006) Planktonic microbial communities associated with fracture-derived groundwater in a deep gold mine of South Africa. Geomicrobiology Journal 23: 475-497.

Lopez-Garcia, P., Superron, S., Philippot, P., Foriel, J., and Moreira, D. (2003) Bacterial diversity in hydrothermal sediment and epsilonproteobacterial dominance in experimental microcolonizers at the Mid-Atlantic Ridge. Environ. Microbiol. 5: 961-976.

Lovley, D.R., and Chapelle, F.H. (1995) Deep subsurface microbial processes. Reviews of Geophysics 33: 365-381.

Lysnes, K., Thorseth, I.H., Steinsbu, B.O., Ovreas, L., Torsvik, T., and Pedersen, R.B. (2004) Microbial community diversity in seafloor basalt from the Arctic spreading ridges. FEMS Microbiol. Ecol. 50: 213-230.

Moyer, C.L., Tiedge, J.M., Dobbs, F.C., and Karl, D.M. (1998) Diversity of deep-sea hydrothermal vent Archaea from Loihi Seamount, Hawaii. Deep-Sea Res. II 45: 303-317.

Nadkarni, M.A., Martin, F.E., Jacques, N.A., and Hunter, N. (2002) Determination of bacterial load by real-time PCR using a broad-range (universal) probe and primers set. Microbiology 148: 257-266.

Norris, P.R., Clark, D.A., Owen, J.P., and Waterhouse, S. (1996) Characteristics of Sulfobacillus acidophilus sp. nov. and other moderately thermophilic mineralsulphide-oxidizing bacteria. Microbiology 142: 775-783.

Page, R.D.M. (1996) TREEVIEW: An application to display phylogenetic trees on personal computers. Computer Applications in the Biosciences 12: 357-358.

Pedersen, K., Ekendahl, S., Tullborg, E.-L., Furnes, H., Thorseth, I., and Tumyr, O. (1997) Evidence of ancient life at $207 \mathrm{~m}$ depth in a granitic aquifer. Geology 25: 827-830.

Posada, D., and Crandall, K.A. (1998) MODELTEST: testing the model of DNA substitution. Bioinformatics 14: 817-818.

Purkhold, U., Wagner, M., Timmermann, G., Pommerening-Roser, A., and Koops, H.P. (2003) 16S rrNA and amoA-based phylogeny of 12 novel betaproteobacterial ammonia-oxidizing isolates: extension of the dataset and proposal of a new lineage within the nitrosomonads. Int. J. Syst. Evol. Microbiol. 53: 1485-1494.

Reysenbach, A.-L., Longnecker, K., and Kirshtein, J. (2000) Novel bacterial and archaeal lineages from an in situ growth chamber deployed at a Mid-Atlantic ridge hydrothermal vent. Appl. Environ. Microbiol. 66: 3798-3806.

Schloss, P.D., and Handelsman, J. (2005) Introducing DOTUR, a computer program for defining operational taxonomic units and estimating species richness. Appl. Environ. Microbiol. 71: 1501-1506.

Schloss, P.D., and Handelsman, J. (2006) Introducing SONS, a tool for operational taxonomic unit-based comparisons of microbial community memberships and structures. Appl. Environ. Microbiol. 72: 6773-6779.

Schmid, M., Walsh, K., Webb, R., Rijpstra, W.I., van de Pas-Schnoonen, K., Verbruggen, M.J. et al. (2003) Candidatus 'Scalindua broadae'. sp. nov. 
Candidatus 'Scalindua wagneri', sp. nov., two new species of anaerobic ammonium oxidizing bacteria. Syst. Appl. Microbiol. 26: 529-538.

Schmidt, J.M., Sharp, W.P., and Starr, M.P. (1982) Metallic-oxide encrustations of the nonprosthecate stalks of naturally occurring populations of Planctomyces bekefii. Curr. Microbiol. 7: 389-394.

Sims, K.W.W., Goldstein, S.J., Blichert-Toft, J., Perfit, M.R., Kelemen, P., Fornari, D.J. et al. (2002) Chemical and isotopic constraints on the generation and transport of magma beneath the East Pacific Rise. Geochim. Cosmochim. Acta 66: 3481-3504.

Sorokin, D.Y., Tourova, T.P., Antipov, A.N., Muyzer, G., and Kuenen, J.G. (2004) Anaerobic growth of the haloalkaliphilic denitrifying sulfur-oxidizing bacterium Thioalkalivibrio thiocyanodentrificans sp. nov. with thiocyanate. Microbiology 150: 2435-2442.

Straub, K.L., Schonhuber, W.A., Buchholz-Cleven, B.E.E., and Schink, B. (2004) Diversity of ferrous iron-oxidizing, nitrate-reducing bacteria and their involvement in oxygen-independent iron cycling. Geomicrobiology Journal 21: 371-378.

Stroncik, N.A., and Schmincke, H. (2001) Evolution of palagonite: Crystallization, chemical changes, and element budget. Geochemistry, Geophysics, Geosystems 2.

Suzuki, Y., Inagaki, F., Takai, K., Nealson, K.H., and Horikoshi, K. (2004) Microbial diversity in inactive chimney structures from deep-sea hydrothermal systems. Microbial Ecology 47: 186-196.

Takai, K., and Horikoshi, K. (2000) Rapid detection and quantification of members of the archaeal community by quantitative PCR using fluorogenic probes. Appl. Environ. Microbiol. 66: 5066-5072.

Takai, K., Suzuki, M., Nakagawa, S., Miyazaki, M., Suzuki, Y., Inagaki, F., and Horikoshi, K. (2006) Sulfurimonas paralvinellae sp. nov., a novel mesophilic, hydrogen- and sulfur-oxidizing chemolithoautotroph within the Epsilonproteobacteria isolated from a deep-sea hydrothermal vent polychaete nest, reclassification of Thiomicrospira denitrificans as Sulfurimonas denitrificans comb. nov. and amended description of the genus Sulfurimonas. Int. J. Syst. Evol. Microbiol. 56: 1725-1733.

Teske, A., Alm, E., Regan, J.M., Toze, S., Rittmann, B.E., and Stahl, D.A. (1994) Evolutionary relationships among ammonia- and nitrite-oxidizing bacteria. $J$. Bacteriol. 176: 6623-6630.

Thorseth, I.H., Torsvik, T., Torsvik, V., Daae, F.L., Pedersen, R.B., and Party, K.-S. (2001) Diversity of life in ocean floor basalt. Earth Planet. Sci. Lett. 194: 31-37.

Torsvik, V., Goksoyr, J., and Daae, F.L. (1990) High diversity in DNA of soil bacteria. Appl. Environ. Microbiol. 65: 782-787.

Tringe, S.G., von Mering, C., Kobayashi, A., Salamov, A.A., Chen, K., Chang, H.W. et al. (2005) Comparative metagenomics of microbial communities. Science 22: 554-557.

Venter, J.C., Remington, K., Heidelberg, J.F., Halpern, A.L., Rusch, D., Knap, J.A. et al. (2004) Environmental genome shotgun sequencing of the Sargasso Sea. Science 304: 66-74. 
Wirsen, C.O., Jannasch, H.W., and Molyneaux, S.J. (1993) Chemosynthetic microbial activity at Mid-Atlantic Ridge hydrothermal vent sites. Journal of Geophysical Research-Solid Earth 98: 9693-9703.

Yue, J.C., and Clayton, M.K. (2005) A similarity measure based on species proportions. Commun. Stat. Theor. Methods 34: 2123-2131.

Zwickl, D.J. (2006) Genetic algorithm approaches for the phylogenetic analysis of large biological sequence datasets under the maximum likelihood criterion. In: The University of Texas at Austin. 


\title{
Chapter 4. The subsurface ocean crust biosphere: Evidence suggesting low biomass in old ( $>10 \mathrm{Ma})$ ocean crust
}

\author{
Cara M. Santelli ${ }^{1}$, Neil Banerjee ${ }^{2}$, Wolfgang Bach ${ }^{3}$, Katrina J. Edwards ${ }^{4 *}$ \\ ${ }^{1}$ MIT/WHOI Joint Program in Oceanography and Ocean Engineering \\ ${ }^{2}$ Department of Earth Sciences, University of Western Ontario, London, Ontario, N6A \\ 5B7, Canada \\ ${ }^{3}$ Fachbereich Geowissenschaften, Universität Bremen, Postfach 3304 40, D-28334 \\ Bremen, Germany \\ ${ }^{4 *}$ Geomicrobiology Group, Department of Biological Sciences, Marine Environmental \\ Biology, University of Southern California, 3616 Trousdale Blvd, Los Angeles, CA, USA
}

\begin{abstract}
The extent to which microorganisms inhabit the ocean crust, both laterally and vertically, is largely undefined. It is hypothesized that redox reactions occurring in igneous crust could support chemosynthetic communities wherever fluid-rock interactions occur. Ocean crust sustains the most extensive aquifer and therefore could harbor the largest endolithic microbial community on the planet. This potentially immense biosphere, however, is just beginning to be examined using molecular biological techniques. This study attempts to identify microbiological communities existing within ocean crust from several different ages, $\sim 15 \mathrm{Ma}, \sim 24 \mathrm{Ma}$, and $\sim 123 \mathrm{Ma}$, to better constrain the boundaries of the subseafloor biotope. Culture-based enrichment techniques and phylogenetic analyses were carried out on drill-core samples of volcanic basement from various locations in the Pacific Ocean, which were obtained on three separate Ocean Drilling Program (ODP) research cruises: Leg 192, Leg 205, and Leg
\end{abstract}


206. The methods and protocols used here have successfully obtained genetic information from young seafloor minerals and rocks (Edwards et al., 2003; Rogers et al., 2003, Chapters 2 and 3). Results from this study, however, failed to provide indisputable evidence for microbial life inhabiting the subseafloor ocean crust. We empirically determined the efficiency and sensitivity of our DNA extraction and amplification protocols and show that our methods are capable of detecting low quantities of biomass. Comprehensively, these analyses suggest that the subsurface biosphere in "old" ocean crust is substantially less relative to "young" seafloor crust and may be below the detection level of most molecular and microbiological techniques.

\subsection{Introduction}

The subsurface biosphere is a topic that has garnered significant interest in the past years. The majority of studies exploring the oceanic deep biosphere have concentrated on deeply buried sediments (Biddle et al., 2006; D'Hondt et al., 2004; Kormas et al., 2003) as well as mid-temperature hydrothermal fluids emanating from the flanks of mid-ocean ridges (MORs) (Cowen et al., 2003; Huber et al., 2003; Huber et al., 2006). An array of studies also suggests that a rock-hosted biosphere could extend laterally beyond the MOR environment and extend deep into the ocean crust (Banerjee and Muehlenbachs, 2003; Fisk et al., 1998; Furnes and Staudigel, 1999; Furnes et al., 2001; Giovannoni et al., 1996; Thorseth et al., 1992; Thorseth et al., 2003; Torsvik et al., 1998). In this environment, large volumes of oxygenated seawater interact with the highly permeable and porous basaltic rock, which contains reduced elements such as Fe, 
Mn, and S. Microorganisms can catalyze sluggish redox reactions (as further described in Bach and Edwards (2003) and Edwards et al. (2005)) to gain metabolic energy for growth. Because ocean crust covers $>60 \%$ of the Earth's surface, this basaltic substrate has the potential to support one of the most extensive endolithic communities on the planet. Consequently, even modest amounts of mineral-microbe interactions in the ocean crust could contribute significantly to geochemical processes such as mineral dissolution rates, mineral precipitation, lithification, and elemental cycles over geological time scales.

The majority of evidence supporting the existence of an endolithic community in off-axis, subsurface ocean crust has been limited to petrographic studies of basalt glass from drill cores of volcanic crust, with supporting evidence from isotopic and microbeam chemical analyses. In short, studies using transmitted light microscopy and scanning electron microscopy (SEM) have revealed either irregular granular or tubular, segmented channels observed throughout the alteration front and extending into fresh glass (Banerjee and Muehlenbachs, 2003; Fisk et al., 1998; Furnes et al., 2001; Thorseth et al., 1995). These features have been observed in a wide variety of geographical settings and geological environments (depth, crustal age, etc). Geochemical analyses of these alteration features commonly reveal an enrichment of biologically relevant elements such as $\mathrm{C}, \mathrm{N}, \mathrm{P}, \mathrm{S}$, and $\mathrm{K}$ relative to fresh, unaltered glass. Additionally, stable carbon isotope analyses of carbonates disseminated within the altered, bulk rock reveal depleted $\delta^{13} \mathrm{C}$ values compared to either fresh basalt or expected inorganically precipitated marine carbonate values. These anomalous alteration patterns and geochemical signatures have 
been interpreted to be a consequence of microbial activity within the ocean crust (Banerjee and Muehlenbachs, 2003; Fisk et al., 1998; Furnes and Staudigel, 1999; Furnes et al., 2001; Giovannoni et al., 1996; Thorseth et al., 1992; Thorseth et al., 2003; Torsvik et al., 1998). This hypothesis, however, is subject to debate as there is a lack of direct evidence (for example, laboratory based studies) linking any microbe with the capability of producing such alteration textures or signatures in basalt glass. Neither abiotically nor biologically mediated laboratory experiments carried out thus far have been successful at replicating these long (tens to hundreds of micrometers long), tubular alteration features (Staudigel et al., 1995; Thorseth et al., 1995) in glass.

Several recent Ocean Drilling Program (ODP) research cruises have attempted to integrate micro- and molecular biological studies with the more standard geochemical and petrological analyses of igneous basement drill cores to determine the extent of the subsurface biosphere in older, off-axis ocean crust (Fisk et al., 2000; Giovannoni et al., 1996; Lysnes et al., 2004b). On the first two of these research drilling cruises (Fisk et al., 2000; Giovannoni et al., 1996), researchers were either entirely unable to identify rockhosted, microbial communities using molecular biological techniques (Fisk et al., 2000; Giovannoni et al., 1996) or have issued caution in linking the phylogenetic affiliations (in which the sequences have never been published in a public database) with the basalt community (Fisk et al., 2000; Giovannoni et al., 1996). However, in a later study from the Southeast Indian Ridge (Lysnes et al., 2004b), researchers successfully cultured bacteria and archaea as well as extracted and amplified bacterial DNA from 18-28 Ma ocean crust for analyses. Sequences were retrieved from 6 major phylogenetic bacterial 
groups, including the Gamma- and Beta- Proteobacteria, the CFB group, Actinobacteria, Green nonsulfur bacteria, and the Bacillus/Clostridium group. It was additionally concluded through enrichment studies that Fe-reduction and methanogenesis are important physiologies for microbial communities residing in the deep subsurface. The degree to which drilling related contamination influenced their findings, however, was not directly addressed in that study. For example, sequences related to green nonsulfur bacteria, primarily phototrophic microorganisms, and Pseudoalteromonas flavipulchra, a type of bacterioplankton, were recovered from the subsurface basalts. These microorganisms would not be expected to actively metabolize in a deep subsurface environment where light is not an available energy source.

To date, few reports have indisputably determined the phylogenetic diversity of the subseafloor environment using molecular biological techniques. A single study (Lysnes et al., 2004b), while revealing, is insufficient in describing entire microbial communities that inhabit the ocean crust, the overall extent of this biosphere, and the extent to which these microorganisms impact subseafloor weathering processes. With these and other relevant drilling studies (Reysenbach et al., 1998), it is apparent that many specific challenges (e.g. molecular biological methods and drilling capabilities) are present in studies that attempt to rigorously determine the true nature of rock-hosted microbial communities in the subsurface environment.

In this study, we set out to further elucidate the subsurface endolithic microbial biotope by using both culture-dependent and culture-independent $16 \mathrm{~S}$ rRNA phylogenetic analyses of volcanic basement drill core samples. We collected and 
analyzed basalt rock and glass samples from three different ODP drill sites in the Pacific Ocean, which represent a variety of different environments (in terms of age, likely fluid flux, extent of alteration, depth, etc.) within the ocean crust. Cultivation approaches were also used to enrich for microorganisms with defined physiological capabilities, such as $\mathrm{Fe}$ oxidation, Fe reduction, sulfide oxidation, sulfate reduction, and methanogenesis. These approaches were designed to provide an assessment of the extent of the ocean crust biosphere at these sites, and provide insight into the role that microbial activity plays in crustal evolution.

\subsection{Geological Setting}

Samples were collected on three separate research cruises, Legs 192, 205, and 206, of the Ocean Drilling Program (ODP). More specifically, the volcanic basement drill cores analyzed for this study were obtained from Holes 1184A, 1253, and 1256D. All three drill sites lie in the Pacific Ocean (Figure 4.1), however the geological and physical settings of these localities is variable.

\subsubsection{Hole 1184A}

Hole 1184A is located on the Ontong Java Plateau, the world's largest volcanic oceanic plateau. The volcaniclastic sequence, estimated to have been emplaced at $123 \pm$ 1.8 Ma (Chambers et al., 2004), consists of massive layers of coarse lithic-vitric tuff, lapilli tuff, and lapillistone, with thin interstitial layers of fine ash. The entire cored sequence is altered to varying degrees, but consistently by low-temperature fluids 
(Mahoney et al., 2001). Much of the volcanic glass is completely altered, but unaltered glass shards rimmed with smectite were found in several cores (Mahoney et al., 2001) Petrographic analyses (Banerjee and Muehlenbachs, 2003) show vitric shards containing abundant textural features that are consistent with previously described evidence for microbial alteration. Several of these samples were included in analyses for this study (Table 4.1).

\subsubsection{Hole 1253}

Hole 1253 is located at the Costa Rica subduction zone on the Cocos plate, just $\sim 200 \mathrm{~m}$ seaward of the deformation front. The oceanic crust at this site was formed $\sim 24$ Ma at the East Pacific Rise (EPR). The top of the volcanic unit, which was not sampled for this study, is dominated by a thick sill that intruded sediments. Petrological studies (Morris et al., 2003) indicate that the lower igneous unit could either be a sill complex or a series of slowly cooled lava flows. In general, the cryptocrystalline to microcrystalline basalt contains some fresh glass, which shows incipient alteration to zeolites and clays, particularly at depth. Neither tubular nor granular textures consistent with microbial alteration were identified in thin sections for shipboard analyses. In addition to the glassy lavas, a number of carbonate and zeolite filled veins are also present, and were sampled for this study (Table 4.1). Heat flow anomalies, pore fluid chemistry of sediments from this drill hole and from a nearby drill site (ODP Leg 170) (Silver, 2001), and initial results from the in-situ monitors (CORK IIs - Circulation Obviation Retrofit Kits) 
(Shipboard Scientific Party, 2004) all indicate active fluid flow within the uppermost basement.

\subsubsection{Hole 1256D}

Hole $1256 \mathrm{D}$ is also located on the Cocos plate in ocean crust that was formed at the East Pacific Rise. It has been determined that the superfast spreading rate at the time of crust formation (approximately $15 \mathrm{Ma}$ ) was $\sim 200-220 \mathrm{~mm} / \mathrm{yr}$ (Wilson, 1996). The volcanic stratigraphy is dominated by basaltic sheet flows with chilled margins, massive flows (the most predominant one being at the sediment/basement interface similar to Hole 1253), subordinate flows of pillow lavas, breccias, and rare dikes (Wilson et al., 2003). Hole 1256D has been subsequently deepened to 1507 meters below seafloor through sheeted dikes and into gabbro (Wilson et al., 2006). Independent of subseafloor depth, glassy margins from pillow flows and breccias are commonly altered and veined by carbonate, celadonite, and iron oxyhydroxide. Samples specific to this study were primarily glassy with slight to moderate degrees of alteration (Table 4.1). Initial shipboard examination of thin sections did not reveal any irregular alteration features, only smooth alteration fronts (Wilson et al., 2003). Subsequent work has identified granular alteration textures and depleted carbon isotope values in several hyaloclastite samples (Banerjee, unpub. data).

\subsection{Methods}

\subsubsection{Sample Collection}


Because the drill cores were sampled specifically for microbiological and molecular biological studies, extra care was taken during the recovery and sampling process to minimize and account for contamination. Upon core recovery, the core liner was cut without disturbing the selected core, and sterile tools were used to collect samples for immediate processing and sub-sampling in the microbiology lab. Samples for all DNA analyses were stored at $-80^{\circ} \mathrm{C}$, and all enrichment media was inoculated immediately. A more detailed description of the sampling and sub-sampling procedures can be found in the Explanatory Notes sections for Legs 205 and 206 (Morris et al., 2003; Wilson et al., 2003). Although careful techniques were used at all times in the laboratory, the samples should be treated as potentially contaminated. This is because drilling is not a sterile procedure, in part because surface seawater is used throughout the

process. Both chemical and particulate tracer tests (Smith et al., 2000a; Smith et al., 2000b) were used on Legs 205 and 206 to help quantify contamination. These tests may not be reliable for all instances of drilling in igneous rock (Staudigel et al., 2004), although there has been some success with the use of perfluorocarbon tracers (Lever et al., 2006).

\subsubsection{Enrichment Cultures}

Drillcore sub-samples were crushed using sterile techniques and made into a slurry with artificial seawater as described previously (Morris et al., 2003). The slurry was used as an inoculum for a number of different media types to select for microorganisms of specific autotrophic and heterotrophic physiologies, such as $\mathrm{Fe}$ 
reduction, Fe oxidation, sulfate reduction, sulfide oxidation, and methanogenesis. Each media type started with artificial seawater and was amended with a variety of compounds or minerals as described previously (Morris et al., 2003) to be used as energy sources. A more complex approach was used to enrich for neutrophilic Fe-oxidizing bacteria, which utilized gradient tubes made with $\mathrm{FeS}$ or $\mathrm{FeCO}_{3}$ as described by Emerson and Moyer (1997). Duplicate or triplicate enrichment cultures were started from all samples, except for those obtained from Hole 1184A on ODP Leg 192.

\subsubsection{DNA Extraction}

Samples stored at $-80^{\circ} \mathrm{C}$ for DNA extraction were first fragmented using a sterile hammer and chisel and then ground to a finer particle size with a sterile mortar and pestle. Two different protocols for extracting genomic DNA from basalt samples were used. The first method (protocol PC) involved a lysozyme treatment with numerous freeze-thaw steps and a phenol-chloroform isolation as described previously (Edwards et al., 2000). Other studies on low biomass samples (Webster et al., 2003) suggest that similar freeze-thaw methods are inadequate at extracting either quantifiable or amplifiable (using Polymerase Chain Reaction, PCR) DNA. The second DNA extraction method (protocol MB) utilized the Ultraclean ${ }^{\mathrm{TM}}$ soil DNA kit (MoBio Laboratories Inc.) following the manufacturer's alternative protocol for maximum yields with slight modifications. A 10 -minute heating step at $70^{\circ} \mathrm{C}$ was incorporated as recommended in the kit protocol for cells that are difficult to lyse. Additionally, $200 \mu \mathrm{g}$ of poly-adenylic 
acid (poly A) was added to the lysis mixture as recommended in previous studies (Webster et al., 2003).

\subsubsection{PCR Amplification}

Following extraction, numerous PCR protocols were attempted in order to amplify the 16S rRNA region of the environmental DNA. Variations included one or more of the following: (i) annealing temperature $\left(47^{\circ} \mathrm{C}-52^{\circ} \mathrm{C}\right)$, (ii) primer sets (described below), (iv) template concentration (1-3 $\mu \mathrm{L} / \mathrm{rxn})$, (v) template dilutions (1:1, 1:10, 1:100, 1:1000), (vi) primer concentration $(0.2-0.4 \mu \mathrm{M})$, (vii) Bovine Serum Albumin (BSA) concentration $(0-.5 \mu \mathrm{g} / \mu \mathrm{L})$. Successful amplification of DNA used a partial nested PCR technique. To amplify bacterial DNA, primer set 8F (5'AGAGTTTGATCCTGGCTCAG) and 1492R (5'-GGTTACCTTGTTACGACTT) were used for the initial reaction, followed by primer set $515 \mathrm{~F}$ (5'GTGCCAGCMGCCGCGGTAA) and 1492R. Primer sets 4F (5'TCCGGTTGATCCTGCCRG) and 1492R followed by 4F and 915R targeted archaeal DNA. The protocol for bacterial DNA amplification was: (i) initial denaturation for 5 min at $94^{\circ} \mathrm{C}$, (ii) 20 cycles (initial reaction) or 18 cycles (nested reaction) of $94^{\circ} \mathrm{C}$ ( $1 \mathrm{~min}$ $30 \mathrm{sec}), 47^{\circ} \mathrm{C}(1 \mathrm{~min} 30 \mathrm{sec})$, and $72^{\circ} \mathrm{C}(3 \mathrm{~min})$, and (iii) a final extension for $10 \mathrm{~min}$ at $72^{\circ} \mathrm{C}$. The PCR reaction mixture $(50 \mu \mathrm{L})$ contained $1 \mathrm{X}$ PCR buffer, $0.2 \mathrm{mM}$ PCR nucleotide mix (Promega), $0.2 \mu \mathrm{M}$ of each primer (SigmaGenosys), $1.5 \mathrm{mM} \mathrm{MgCl} 2,1 \mathrm{U}$ of $\operatorname{Taq}{ }^{\circledR}$ DNA polymerase (Promega), and $1 \mu \mathrm{L}$ of template. All PCR reactions were 
carried out using an iCycler thermal cycler (Bio-Rad Laboratories) or a Thermocycler 2400 (Applied Biosystems). PCR products were visualized using gel electrophoresis.

A PCR kill control was also performed on both the environmental samples and the extraction efficiency controls. Kill controls were used to ensure the absence of a PCR inhibitor (in the case of template not amplifying) and were done both prior to, and following a PCR purification procedure using the QIAquick nucleotide removal kit (Qiagen). For each reaction, $1 \mathrm{uL}$ of each the positive control (purified genomic E. coli DNA) and the template DNA was added to the PCR reaction. If the PCR reaction successfully amplified with both the genomic and template DNA, it can be assumed that there was no inhibitory substance in the template DNA.

\subsubsection{Extraction/Amplification Efficiency Tests}

In order to test the efficiency and sensitivity of the two DNA extraction methods and PCR amplification protocol used on our samples, control extractions utilizing a pure culture and sterilized substrates relevant to the composition of ocean crust were also performed. The extraction methods were tested with a pure culture of Marinobacter aquaeolei and two different materials: basaltic glass from the EPR (both methods PC and MB) and a nontronite reference clay from Washington, provided by the Source Clay Minerals Repository at the University of Missouri, (method MB only). The fresh EPR basaltic glass is representative of the majority of samples analyzed in this study. The nontronite control represents the more altered, clay rich basalt samples and clay-filled veins. Immediately prior to inoculating with cells, the control materials were sterilized 
by soaking in $100 \%$ ethanol and autoclaving. These minerals were then washed in a dilute acid to remove excess oxides and rinsed several times in sterile, distilled water. In each control, a known quantity of biomass (ranging from $10^{3}-10^{7}$ cells) was incubated overnight on 1 gram of each of the materials (basalt glass or clay) and frozen overnight at $-80^{\circ} \mathrm{C}$ before performing DNA extractions. The freezing step was included to more closely emulate the conditions in which the environmental samples were handled prior to processing. DNA was then extracted from the entire experiment (basalt chips and culture) with the two different methods and amplified using protocols outlined above.

\subsubsection{Clone Library Construction and Sequencing}

Positively amplified PCR products were purified using the QIAquick ${ }^{\circledR}$ nucleotide removal kit (Qiagen) following the manufacturer's instructions for the cleanup of nonradiogenic samples using centrifugation. Cleaned products were then cloned using the pCR ${ }^{\circledR 2.1-T O P O}{ }^{2} v e c t o r($ Invitrogen) and clone libraries constructed as described in a previous publication (Rogers et al., 2003). Plasmid extractions were done by alkaline lysis at the Keck Facility of the Josephine Bay Paul Center (JBPC), Marine Biological Laboratories (MBL). Sequencing reactions were carried out using the primer M13F (5'GTAAAACGACGGCCAG) and the ABI v 3.1 BigDye terminator sequencing kit (Applied Biosystems). Reactions were analyzed on an ABI3730XL capillary sequencer at the JBPC.

Chromatograms generated by sequencing were viewed and manually edited using the 4Peaks (v1.5) freeware program. The edited sequences were imported into the 
BLAST nucleotide search program through the National Center for Biotechnology Information (NCBI) website (http://www.ncbi.nlm.nih.gov/BLAST/). These searches were performed to find closely related sequences within the GenBank database. Sequences will be submitted to the GenBank data base as soon as the manuscript is submitted for review.

\subsection{Results}

\subsubsection{DNA extraction/amplification efficiency experiments}

Results from the extraction experiment controls (Table 4.2) indicate that method $\mathrm{MB}$ is more efficient at extracting amplifiable genomic DNA from low biomass basalt samples compared to method PC. In experiments with basalt glass as the substrate, method PC was entirely incapable of extracting DNA from Marinobacter aquaeolei suitable for PCR in those experiments containing less than $10^{7}$ cells/g basalt glass. Method MB however, which utilizes a more aggressive bead-beating technique to break open cell walls, was more proficient at extracting amplifiable genomic DNA from as little as $\sim 10^{4}$ cells/g basalt glass. During the extraction procedure, the genomic DNA was eluted into a total volume of $50 \mu \mathrm{L}$. Assuming the extraction method was $100 \%$ efficient and taking into consideration the PCR protocol, which used $1 \mu \mathrm{L}$ of a 100 -fold diluted extraction product, PCR products were obtained by effectively amplifying as few as $\sim 10$ cells. Dilutions of more than (1:100) did not yield any amplification products, suggesting that the DNA was diluted to extinction. In experiments using nontronite as the mineral substrate, the MB method was completely ineffective at extracting amplifiable genomic 
DNA from clay materials. As in the environmental samples, kill controls were successful in showing that PCR inhibiting compounds were absent in all extraction method experiments.

\subsubsection{Environmental Samples}

Environmental DNA extractions were performed on 35 drill core samples using both the PC and MB methods. DNA was not visible for any of the samples by gel electrophoresis. For all variations of extraction protocols, only a single sample amplified DNA with confidence (repeated sample amplifications were consistently successful) from the starting set of 35 samples. This single, successfully amplified sample, in which only bacterial DNA could be identified, was from ODP Site 1184A, Core 45. Kill controls on all samples were effective in amplifying DNA, indicating the absence of PCR inhibitory substances.

\subsubsection{Phylogenetic Analyses}

A bacterial clone library was constructed from the ODP Site 1184A, Core 45 sample. Sequencing results of 34 clones are summarized in Table 4.3. The rRNA sequences from the clone library are compared to other sequences available in the GenBank database by using BLAST analyses (Altschul et al., 1997). These results show that the clone library is composed of representative species from the $\alpha-, \beta-$, and $\gamma$ Proteobacteria phylogenetic groups, as well as the CFB (Cytophaga-FlavobacteriumBacteroides) group. Clones belonging to the $\gamma$ - Proteobacteria group have closest 
relatives that are all isolated and cultured microorganism. Of these clones, 4 different phylotypes emerge. Three belong to the genera Acinetobacter and one closely matches a plant pathogenic Pantoea species. The three Acinetobacter species could potentially be pathogens as well, as they were isolated from a mosquito midgut, human clinical species, and from human subgingival dental plaque. The majority of clones are closely related $(\geq 97 \%)$ to cultured organisms, including those isolated from human clinical species, activated sludge, an estuary, onion seed, and drinking water. The majority of clones belonging to the $\beta$-Proteobacteria group are most closely related to other environmental clones; for example, from a biodegraded oil reservoir, a deep-sea octacoral, a PCE contaminated site, and a river in India. Interestingly, 3 clones are most closely related to environmental sequences obtained from groundwater emanating from a terrestrial, deep subsurface fracture zone in a South African gold mine characterized by a high energy, low diversity microbial biome (Lin et al., 2006b). Several of the other $\beta$-Proteobacteria clones are most similar to a denitrifying bacterium isolated from activated sludge at a wastewater treatment facility. The single $\alpha$-Proteobacteria clone is also most closely related to a clone from the South African mine (Lin et al., 2006b), and is also related to several different Sphingomonas species. All 8 clones belonging to the CFB group appear to be dominated by a single phylotype which is most closely related to an environmental clone extracted from water just downstream from equine manure. These clones are also closely related to numerous Bacteroidetes species isolated from both Arctic and Antarctic sea ice (Brinkmeyer et al., 2003). 


\subsubsection{Enrichment cultures}

Enrichment cultures were set up from subsets of all drill core samples listed in Table1, except for those samples from ODP Leg 192, Hole 1184A. These enrichments were designed to target microorganisms with physiological capabilities that are energetically favorable in the subseafloor ocean crust environment, for example $\mathrm{Fe}$ oxidation or reduction, sulfate reduction, sulfide oxidation, and methanogenesis (Bach and Edwards, 2003). However, not one single enrichment culture of the numerous cultures started succeeded in producing microbial growth.

\subsection{Discussion}

\subsubsection{Protocol Development and Extraction Method Experiments}

The difficulties encountered in extracting and amplifying environmental DNA from subsurface lavas first suggested that either our methods were not proficient for the sample type or that there is a nominal biomass present in old subsurface ocean crust obtained from the study sites. Method PC and the nested PCR protocols, however, were highly successful on weathered sulfide samples obtained from the seafloor (Rogers et al., 2003). Furthermore, method MB has been shown to be very effective in extracting environmental DNA from other volcanic lavas (Chapters 2 and 3). In that study in which the phylogenetic diversity of microorganisms inhabiting young ocean crust exposed at the seafloor was examined, lavas were collected from the East Pacific Rise in the vicinity of $9^{\circ} \mathrm{N}$ by submersible. Basaltic glass samples ranged from fresh (no visible oxide products) to slightly weathered (glass altered to palagonite and clay, oxide crusts on 
surfaces). Method MB was used to extract environmental DNA, however nested PCR was not performed on these samples because all samples readily amplified with primer set 8F-1492. The dramatic difference in ease of PCR amplification protocol between the two different studies (young seafloor EPR lavas vs. old subsurface ocean crust) was our first indication that the biomass in older (likely $>10 \mathrm{Ma}$ ) subsurface ocean crust is considerably less than in young crust.

To test the efficiency of extracting DNA from rocky substrates and ensure that our techniques were sensitive to low biomass, our extraction methods and PCR protocols were tested with active cultures and substrates relevant to the subseafloor. These experiments also gave us a theoretical lower limit of the number of cells which were able to be detected with each of the methods. Our results (Table 4.2) show that method MB is sensitive for glassy lavas with low biomass, however a critical mass (on the order of $10^{4}$ cells total/g material) is necessary in order for the extraction method to be effective. This biomass is comparable to that found in ambient bottom seawater $\left(\sim 10^{4}\right.$ cells $/ \mathrm{mL}$ seawater). Most studies that perform molecular or microbiological biological analyses on deep seawater, however, highly concentrate the biomass on filters using several liters of fluid (e.g., Cowen et al., 2003; DeLong et al., 2006)

Based on our extraction efficiency experiments with nontronite, however, we observed that the presence of clays may dramatically decrease the sensitivity of our methods. A potential explanation for this decrease is that once the cells are lysed the DNA binds tightly to the clay surface in the presence of cations, such as $\mathrm{Na}^{+}$from either the seawater medium or the sodium dodecyl sulfate reagent used in the MoBio extraction 
kit. Subsequently, the DNA is not released from the mineral surfaces during the extraction procedure prior to amplification. This behavior has been observed in numerous studies (Alvarez et al., 1998; Franchi et al., 2003) where negatively bound clay minerals rapidly and tightly bind similarly charged nucleic acids in the presence of cations, which serve as a "link" between the two materials. Another possible explanation is that a high concentration of metals either adsorbed to the clays or incorporated into their structures is being released into solution during the DNA extraction protocol. Metals such as Fe can inhibit the PCR reaction. Because the kill control PCR reactions were successful in amplifying DNA from the nontronite experiments (suggesting the absence of an inhibitor), the more favorable explanation is that the DNA is lysed from the cells and subsequently adsorbed to the clay mineral surfaces during the extraction protocol.

Additionally, it has been suggested that greater sample volumes are required for DNA extractions from low biomass samples. If the basalts are highly altered and contain abundant clays, then using more sample with low cell densities could exacerbate the situation. This will continue to be an issue in future studies trying to find the limits of the ocean crust biosphere, as the clay fraction of basalt increases considerably with age of the crust. Protocols may need to be optimized for inactivating the clay surfaces during the DNA extraction procedure.

\subsubsection{Phylogenetic Analyses}


Analyses of the clone library constructed from the single sample in which DNA was obtained (Hole 1184A, Core 45) show a nominally diverse bacterial community. As can be seen in Table 4.1, this sample also contained abundant tubular alteration features extending from the alteration front into the fresh glass. These anomalous alteration features are similar to those described by Banerjee and Muehlenbachs (2003). Representative clones from only 4 different phylogenetic classes were obtained. Of serious concern is the large number of clones with closest relatives or high degrees of similarity $(\geq 97 \%)$ to cultured microorganisms. Typically in studies from poorly researched or unique environments, the vast majority of clones is most similar to other environmental clones and only distantly related to any isolated microbes (Nercessian et al., 2005; Skidmore et al., 2005). These results may suggest that the samples may have been compromised, possibly by the drilling process.

A more in-depth analysis of the most closely related microorganisms further supports the hypothesis of drilling contamination in this sample. Specifically, all of the related cultured representatives appear to be obligate heterotrophs. Many of the clones are related to various Acinetobacter species, which are generally considered to be pathogenic bacteria. Several other clones are related to bacteria isolated from carbon rich environments that suggest their role in biodegradation; for example, a clone belonging to $\alpha$-Proteobacteria (ODP1184-45B49) is 97\% similar to a Sphingomonas species that are, as a group, chemoheterotrophic bacteria generally known for their biodegrative capabilities. Additionally, two $\beta$-Proteobacteria clones (ODP1184-45B9 and -45B13) are 99\% similar to Diaphrobacter nitroreducens (Khan et al., 2002), which is a poly-carbon 
degrading denitrifying bacterium isolated from activated sludge. While it is not completely unexpected to find chemoheterotrophic microorganisms inhabiting the deep subsurface, it is expected that the complex organic compounds required for growth of these types of biodegrative organisms would be generated by chemolithoautotrophs serving as the primary producers in the subsurface environment (Shock and Holland, 2004).

Additional support for the interpretation of our results comes from our comparative study at the EPR, in which contamination is of little concern due to the sampling techniques which utilize specially designed bioboxes to recover the samples from the seafloor by submersible (Chapter 2). In this study, phylogenetic analyses of young, seafloor lavas depict a very different microbial community. Highly diverse, yet distinct populations of bacteria inhabit these lavas, and this diversity increases with increased basalt glass alteration. Only a minimal portion of the observed diversity can be attributed to deep seawater microbial communities. Although physiology can only be inferred from the phylogeny in a few cases, our results suggest that both lithoautotrophic and heterotrophic communities exist on these surfaces in a generally organic carbon-poor environment. This implies that these autotrophic bacteria could be deriving their energy from the oxidation of the basalt glass, thus supporting a more complex (more productive) community. Further phylogenetic analyses revealed that the majority of the clones from the younger basalt samples are most similar to other highly relevant environmental clones, and often only distantly related to any isolated microorganisms. These results are 
more in-line with what other relevant environmental studies find in molecular biological analyses.

While the phylogenetic analyses of our findings from the drill-core sample relative to the EPR seafloor samples are strongly suggestive of drilling-related contamination, it is also difficult to rule out the possibility that the results are real. Specifically, 4 of the total clones were most closely related to other environmental clones from the terrestrial deep subsurface and several others were most similar to clones from a deep-sea coral specimen. Regardless, we feel that the sample may have been compromised from the drilling process and even the more promising results should be examined with caution.

\subsubsection{Enrichment cultures}

Media was designed to enrich for microbes with physiologies capable of existing and growing in this light-absent, oligotrophic environment, such as those that gain energy by catalyzing redox reactions with $\mathrm{Fe}$ or $\mathrm{S}$. All inoculated media failed to produce microbial growth. Unfortunately, enrichment cultures were never started with samples collected from Hole 1184a, from which the single successfully sequenced sample was obtained. The complete absence of any growth could signify inadequate media design, particularly since the drill core samples have been recovered from great depths (nearly 4 $\mathrm{km}$ below sea level for the majority of samples). However, the same protocol used in this study to isolate Fe-oxidizing bacteria was successfully used in a previous study in which several neutrophilic Fe oxidizers were cultivated from seafloor sulfide minerals (Edwards 
et al., 2003). Additionally, protocols to enrich for Fe-reducers, Fe-oxidizers, and sulfate reducers were successful in obtaining microbial growth from young seafloor lavas from the East Pacific Rise described earlier (Santelli, unpublished results). These enrichment cultures, however, have not been analyzed to determine the physiological capabilities of the microbes. While attempts to isolate microorganisms from subseafloor ocean crust were unsuccessful, the negative results further support our hypothesis that old ocean crust harbors minimal biomass. This interpretation is applied cautiously, however, as steps were not taken to grow these cultures under pressures equivalent to in situ conditions (and sample depths of basalts recovered from the seafloor are at $\sim 2.5 \mathrm{~km}$ compared to $>4$ km depth for subsurface samples).

\subsubsection{Other evidence suggesting minimal biomass}

It has been hypothesized that microbial communities can exist within ocean crust as long as hydrothermal circulation is supported (Staudigel et al., 2004). Heat flow measurements suggest that fluid circulation occurs in crust less than or equal to $\sim 65 \pm \mathrm{Ma}$ (Stein et al., 1995), and porosity and permeability measurements infer circulation to depths up to 200-500 m, e.g. (Alt, 1995). However, it is becoming increasingly apparent that the majority of basalt alteration, an indication of fluid-rock interaction, occurs in young crust on the flank of mid-ocean ridge systems where hydrothermal circulation is most vigorous (Bach and Edwards, 2003; Staudigel et al., 1981; Stein et al., 1995). There is a significant decrease in the permeability of upper ocean crust within the first several million years (Fisher and Becker, 2000) of crustal evolution, suggesting the sealing of 
voids and fractures due to the development of secondary minerals. Age dating of secondary minerals (Staudigel et al., 1981), as well as $\mathrm{Fe}^{3+} / \sum \mathrm{Fe}$ measurements of basalt samples from numerous drill cores (Bach and Edwards, 2003; Johnson and Semyan, 1994), further supports this observation by demonstrating that oxidative alteration occurs primarily in the first 10-15 million years. A petrographic study (Furnes et al., 2001) compared different alteration textures, considered to be either biologically or abiotically induced, in basalt glass from a wide variety of drill cores. Results show that most alteration (a function of the $\%$ of fresh glass remaining regardless of the mechanism) occurs primarily in young crust and increases only slightly in crust $>6 \mathrm{Ma}$.

\subsubsection{Unique approaches to access the deep subsurface}

In an attempt to minimize or completely bypass the complexities associated with drilling, numerous studies have utilized unique approaches to explore the microbial communities inhabiting younger subseafloor lavas. Several recent studies looked at the bacterial (Huber et al., 2003) and archaeal (Huber et al., 2002) communities in diffuseflow hydrothermal fluids emerging from a seamount on the Juan de Fuca Ridge. Both particle-attached and free-living microorganisms were analyzed from the fluids to decipher the subseafloor microbial community. In a related study (Huber et al., 2006), a novel sampling device was designed to directly sample fluids as they emerged from the 3.5 Ma crust with minimal contamination from the overlying sediment and water column. A similar study analyzed basement fluids in a borehole (ODP Leg186, Hole 1026B) from the Juan de Fuca Ridge flank to examine the phylogenetic diversity of $3.5 \mathrm{Ma}$ crust 
(Cowen et al., 2003). Here, a CORK (Circulation Obviation Retrofit Kits) long-term monitoring observatory was retrofitted with a sampling device to collect fluid samples specifically for micro- and molecular biological studies. The results from all of these studies can be used to infer possible microbial communities in older and/or deeper ocean crust. Phylogenetic analyses of these communities are similar, yet distinct, from studies of young seafloor lavas (Chapter 2 and Lysnes et al., 2004a), but are vastly different than those analyzed in this study.

While these unique approaches have provided invaluable information regarding the ocean crust biosphere, this information is limited in the sense that the microorganisms are collected from subseafloor fluid samples. Most microorganisms, particularly in oligotrophic environments, adhere strongly to surfaces. By analyzing only the free-living microorganisms of this biosphere as in the case of the CORK fluid samples, the potentially largest portion of the overall community could remain unresolved. Additionally, in order to determine if and how microbial activity contributes to the evolution of the ocean crust and overlying water column, actual rock samples must be obtained and analyzed with a combination of geochemical and molecular biological techniques.

\subsection{Conclusions}

While not producing any conclusive evidence of a subseafloor biosphere, the cumulative results of this study, in combination with our previous studies in young ocean crust (Chapters 2 and 3), suggest that microbial life may be scarce in deep and old 
igneous basement in the oceans. These findings are further substantiated by physical and geochemical analyses of fluid fluxes and basalt alteration products with respect to evolution of the crust. In many cases, examination of this diminutive biosphere is greatly hindered by the difficulty in accessing this deep, rocky environment as well as insufficient molecular biological tools capable of discerning microbial communities from such a habitat.

The sensitivity and efficiency of protocols used here, however, do not indicate an absence of rock-hosted communities inhabiting the subsurface ocean crust. Ridge flank and off-axis hydrothermal circulation, although considerably less than at mid-ocean ridges, still carries fluids rich in electron acceptors and micronutrients to the crust where energy-yielding redox reactions can support microbial growth. It is recommended that innovative approaches, e.g. the borehole sample device employed by Cowen et al. (2003), be considered and further developed to obtain rock samples. The low biomass samples could also be an outstanding candidate for novel techniques in molecular biology, such as those used in the genomic sequencing of extinct cave bears (Noonan et al., 2005). Although the biomass in crust $\geq 15$ Ma may be less that anticipated, even nominal mineral-microbe interactions in this expansive environment could significantly impact the geochemical cycling of numerous elements in the oceans and in the crust.

\subsection{Acknowledgements}

The authors thank the Co-Chief scientists and staff scientists for ODP Legs 192, 205, and 206 for accommodating and facilitating the sampling techniques required for 
microbiological and molecular biological analyses. This research used samples and data provided by the Ocean Drilling Program (ODP). ODP was sponsored by the NSF and participating countries under management of Joint Oceanographic Institutions (JOI), Inc. Funding for this research was provided by a Schlanger Ocean Drilling Fellowship to C.M.S. and U.S.S.S.P Post-Cruise Award \#TX A\&M F001724 to C.M.S. and K.J.E. Participation for NB on ODP Legs 192 and 206 was provided by Canada ODP. 


\subsection{Tables}

\section{Table 4.1. Sample locations and descriptions}

\begin{tabular}{|c|c|c|c|c|}
\hline $\begin{array}{l}\text { ODP } \\
\text { Leg }\end{array}$ & Hole & Core & $\begin{array}{l}\text { Depth } \\
\text { (mbsf) }\end{array}$ & Sample Description \\
\hline 192 & $1184 \mathrm{~A}$ & 13 & 224.57 & glass shards with abundant tubular alteration textures \\
\hline 192 & $1184 \mathrm{~A}$ & 39 & 471.71 & glass shards with granular alteration textures \\
\hline 192 & $1184 \mathrm{~A}$ & 45 & 520.64 & glass shards with granular and small tubular textures \\
\hline 205 & 1253 & 32 & 543.33 & unaltered microcrystalline basalt, clay-filled veins \\
\hline 205 & 1253 & 33 & 549.40 & microcrystalline basalt, slightly altered to clay/zeolite \\
\hline 205 & 1253 & 34 & 554.73 & microcrystalline basalt, slight alteration, green clay-filled veins \\
\hline 205 & 1253 & 36 & 563.00 & microcrystalline basalt, slight alteration \\
\hline 205 & 1253 & 37 & 566.30 & microcrystalline basalt, veins filled with clay, zeolite, and altered glass \\
\hline 205 & 1253 & 38 & 573.51 & microcrystalline basalt, very slight alteration, thin veins \\
\hline 205 & 1253 & 39 & 578.50 & microcrystalline basalt, small fractures \\
\hline 205 & 1253 & 41 & 587.05 & microcrystalline basalt, unaltered \\
\hline 205 & 1253 & 42 & 592.40 & microcrystalline basalt, rare alteration, green clay-filled veins \\
\hline 205 & 1253 & 43 & 598.73 & $\begin{array}{l}\text { microcrystalline basalt, veins filled with green clay, cryptocrystalline } \\
\text { groundmass, altered glass }\end{array}$ \\
\hline 206 & 1256 & 4 & 286.30 & slightly altered, fine-grained massive basalt, saponite filled veins \\
\hline 206 & $1256 \mathrm{D}$ & 6 & 298.54 & slightly altered, fine-grained massive basalt, saponite filled veins \\
\hline 206 & $1256 \mathrm{D}$ & 8 & 318.37 & slightly altered, fine-grained massive basalt, saponite filled veins \\
\hline 206 & $1256 \mathrm{D}$ & 11 & 335.52 & slightly altered, fine-grained massive basalt, saponite filled veins \\
\hline 206 & $1256 \mathrm{D}$ & 14 & 361.30 & $\begin{array}{l}\text { cryptocrystalline basalt with glassy margins, moderately altered glass, } \\
\text { saponite and celadone-filled veins }\end{array}$ \\
\hline 206 & $1256 \mathrm{D}$ & 15 & 364.33 & $\begin{array}{l}\text { cryptocrystalline basalt with glassy margins, highly altered glass, saponite } \\
\text { and celadonite-filled veins } \\
\text { slightly altered cryptocrystalline basalt }\end{array}$ \\
\hline 206 & $1256 \mathrm{D}$ & 18 & 378.38 & glassy volcanic breccia, highly altered glass shards \\
\hline 206 & $1256 \mathrm{D}$ & 21 & 398.27 & slightly altered cryptocrystalline basalt with glassy margins, saponite filled \\
\hline 206 & $1256 \mathrm{D}$ & 22 & 409.88 & veins \\
\hline 206 & $1256 \mathrm{D}$ & 24 & 420.52 & slightly altered cryptocrystalline basalt \\
\hline 206 & $1256 \mathrm{D}$ & 26 & 440.26 & slightly altered cryptocrystalline basalt \\
\hline 206 & $1256 \mathrm{D}$ & 30 & 461.69 & moderately altered hyaloclastite breccia, slightly altered glass \\
\hline 206 & $1256 \mathrm{D}$ & 33 & 481.08 & slightly altered cryptocrystalline basalt, saponite-filled veins \\
\hline 206 & $1256 \mathrm{D}$ & 40 & 517.72 & cryptocrystalline basalt with slightly altered glassy margins \\
\hline 206 & $1256 \mathrm{D}$ & 43 & 534.09 & glass clasts within an altered glassy matrix \\
\hline 206 & $1256 \mathrm{D}$ & 46 & 563.91 & slightly altered cryptocrystalline basalt, large vesicles \\
\hline 206 & $1256 \mathrm{D}$ & 50 & 592.32 & slightly altered cryptocrystalline basalt, glassy margins \\
\hline 206 & $1256 \mathrm{D}$ & 51 & 598.28 & $\begin{array}{l}\text { moderately altered hyaloclastite breccia, fresh to slightly altered glass shards } \\
\text { slightly altered microcrystalline basalt }\end{array}$ \\
\hline 206 & $1256 \mathrm{D}$ & 60 & 669.75 & slightly altered cryptocrystalline basalt \\
\hline 206 & $1256 \mathrm{D}$ & 62 & 687.29 & slightly altered cryptocrystalline basalt with glassy margins, saponite filled \\
\hline 206 & $1256 \mathrm{D}$ & 70 & 729.18 & veins \\
\hline 206 & $1256 \mathrm{D}$ & 74 & 747.25 & slightly altered cryptocrystalline basalt \\
\hline
\end{tabular}


Table 4.2. DNA Extraction/Amplification Efficiency Results

\begin{tabular}{lccccc}
\hline \multirow{2}{*}{$\begin{array}{l}\text { Biomass } \\
\text { cells g }^{-1} \text { material) }\end{array}$} & \multicolumn{2}{c}{ Method PC } & & \multicolumn{2}{c}{ Method MB } \\
\cline { 2 - 3 } \cline { 6 - 6 } & Gasalt & Clay & & Basalt & Clay \\
Glass & \\
\hline$\sim 10^{3}$ & No $^{\mathrm{a}}$ & $\mathrm{NA}^{\mathrm{b}}$ & & No & No \\
$\sim 10^{4}$ & No & NA & & Yes & No \\
$\sim 10^{5}$ & No & NA & & Yes & No \\
$\sim 10^{6}$ & No & NA & & Yes & No \\
$\sim 10^{7}$ & Yes & NA & & Yes & No \\
\hline
\end{tabular}

a. Indication of successful DNA amplification.

b. Experiment wasn't performed. 
Table 4.3. Clone library results.

\begin{tabular}{|c|c|c|c|c|}
\hline Clone & $\begin{array}{l}\text { Phylogenetic } \\
\text { group }\end{array}$ & $\begin{array}{c}\text { Closest aligned GenBank } \\
\text { sequence (organism or clone) }\end{array}$ & $\begin{array}{c}\% \\
\text { similarity } \\
\end{array}$ & $\begin{array}{l}\text { Description of most } \\
\text { related isolate/clone }\end{array}$ \\
\hline ODP1184-45B1 & $\gamma$-Proteobacteria & Acinetobacter junii (AF417863) & $98 \mathrm{n}$ & microbiota of a mosquito midgut \\
\hline ODP1184-45B2 & $\mathrm{CFB}$ & clone $150 \mathrm{ds} 20(\mathrm{AY} 212601)$ & 97 & water downstream of equine manure \\
\hline ODP1184-45B3 & $\gamma$-Proteobacteria & Pantoea sp. BD 336 (AY530794) & 98 & onion seed pathogen \\
\hline ODP1184-45B8 & $\gamma$-Proteobacteria & Acinetobacter parvus(AJ293691) & 98 & $\begin{array}{l}\text { small-colony-forming species from } \\
\text { human clinical specimens }\end{array}$ \\
\hline ODP1184-45B9 & $\beta$-Proteobacteria & clone PL-30B5 (AY570616) & 99 & $\begin{array}{l}\text { low-temperature biodegraded } \\
\text { Canadian oil reservoir }\end{array}$ \\
\hline ODP1184-45B11 & $\beta$-Proteobacteria & clone ctg_NISA211 (DQ396042) & 97 & cloned from deep-sea octacoral \\
\hline ODP1184-45B12 & CFB & clone $150 \mathrm{ds} 20(\mathrm{AY} 212601)$ & 97 & water downstream of equine manure \\
\hline ODP1184-45B13 & $\beta$-Proteobacteria & clone PL-30B5 (AY570616) & 97 & $\begin{array}{l}\text { low-temperature biodegraded } \\
\text { Canadian oil reservoir }\end{array}$ \\
\hline ODP1184-45B15 & $\beta$-Proteobacteria & clone MP104-SW-b5 (DQ088795) & 98 & $\begin{array}{l}\text { high energy, low diversity crustal } \\
\text { biome }\end{array}$ \\
\hline ODP1184-45B16 & $\gamma$-Proteobacteria & Acinetobacter parvus(AJ293691) & 99 & $\begin{array}{l}\text { small-colony-forming species from } \\
\text { human clinical specimens }\end{array}$ \\
\hline ODP1184-45B22 & $\gamma$-Proteobacteria & Pantoea sp. BD 336 (AY530794) & 98 & onion seed pathogen \\
\hline ODP1184-45B24 & $\gamma$-Proteobacteria & $\begin{array}{l}\text { Acinetobacter genomosp. } \\
\text { C1(AY278636) }\end{array}$ & 98 & $\begin{array}{l}\text { microflora associated with human } \\
\text { dental caries }\end{array}$ \\
\hline ODP1184-45B26 & CFB & clone $150 \mathrm{ds} 20(\mathrm{AY} 212601)$ & 97 & water downstream of equine manure \\
\hline ODP1184-45B27 & $\beta$-Proteobacteria & CLi28 (AF529329) & 98 & $\begin{array}{l}\text { uncultured beta-proteobacteria from } \\
\text { PCE-contaminated site }\end{array}$ \\
\hline ODP1184-45B29 & $\beta$-Proteobacteria & $\begin{array}{l}\text { Burkholderia sp. clone AK168 } \\
\text { (AY005032) }\end{array}$ & 98 & $\begin{array}{l}\text { isolated from human subgingival } \\
\text { dental plaque }\end{array}$ \\
\hline ODP1184-45B35 & $\gamma$-Proteobacteria & Pantoea sp. BD 336 (AY530794) & 99 & onion seed pathogen \\
\hline ODP1184-45B36 & CFB & clone & 98 & water downstream of equin \\
\hline ODP1184-45B40 & $\beta$-Proteobacteria & $\begin{array}{l}\text { Acidovorax sp. R-24607 } \\
\text { (AM084011) }\end{array}$ & 99 & denitrifying bacteria \\
\hline ODP1184-45B43 & $\gamma$-Proteobacteria & Pantoea sp. BD 336 (AY530794) & 99 & onion seed pathogen \\
\hline ODP1184-45B44 & CFB & clone $150 \mathrm{ds} 20$ (AY21 & 98 & water downstream of equine manure \\
\hline ODP1184-45B48 & $\beta$-Proteobacteria & $\begin{array}{l}\text { Acidovorax sp. R-24607 } \\
\text { (AM084011) }\end{array}$ & 99 & denitrifying bacteria \\
\hline ODP1184-45B49 & $\alpha$-Proteobacteria & $\begin{array}{l}\text { clone MP104-0927-b68 } \\
\text { (DQ088806) }\end{array}$ & 99 & $\begin{array}{l}\text { high energy, low diversity crustal } \\
\text { biome }\end{array}$ \\
\hline ODP1184-45B53 & CFB & clone $150 \mathrm{ds} 20(\mathrm{AY} 212601)$ & 98 & water downstream of equine manure \\
\hline ODP1184-45B54 & $\beta$-Proteobacteria & $\begin{array}{l}\text { Acidovorax sp. R-24607 } \\
\text { (AM084011) }\end{array}$ & 98 & denitrifying bacteria \\
\hline ODP1184-45B59 & $\beta$-Proteobacteria & $\begin{array}{l}\text { Acidovorax sp. R-24607 } \\
\text { (AM084011) }\end{array}$ & 99 & denitrifying bacteria \\
\hline ODP1184-45B63 & $\beta$-Proteobacteria & clone SB108 (AY193940) & 97 & $\begin{array}{l}\text { microbial diversity in water from } \\
\text { Mula-Mutha river in India }\end{array}$ \\
\hline ODP1184-45B64 & CFB & clone $150 \mathrm{ds} 20$ (AY212601) & 97 & water downstream of equine manure \\
\hline ODP1184-45B69 & $\beta$-Proteobacteria & clone MP104-SW-b5 (DQ088795) & 99 & $\begin{array}{l}\text { high energy, low diversity crustal } \\
\text { biome }\end{array}$ \\
\hline ODP1184-45B72 & $\beta$-Proteobacteria & clone ctg_NISA211 (DQ396042) & 99 & cloned from \\
\hline ODP1184-45B77 & $\gamma$-Proteobacteria & Acinetobacter parvus(AJ293691) & 99 & $\begin{array}{l}\text { small-colony-forming species from } \\
\text { human clinical specimens }\end{array}$ \\
\hline ODP1184-45B80 & $\gamma$-Proteobacteria & Acinetobacter parvus(AJ293691) & 98 & $\begin{array}{l}\text { small-colony-forming species from } \\
\text { human clinical specimens }\end{array}$ \\
\hline ODP1184-45B81 & $\beta$-Proteobacteria & clone MP104-SW-b5 (DQ088795) & 99 & $\begin{array}{l}\text { from a high energy, low diversi1 } \\
\text { crustal biome }\end{array}$ \\
\hline ODP1184-45B88 & CFB & clone $150 \mathrm{ds} 20$ (AY212601) & 98 & $\begin{array}{l}\text { from water downstream of } \\
\text { equine manure }\end{array}$ \\
\hline & $\gamma$-Proteobacteria & Acinetobacter junii (AF417863) & 99 & microbiota of a mosquito midgu \\
\hline ODP1184-45B92 & $\beta$-Proteobacteria & clone ctg_NISA211 (DQ396042) & 99 & cloned from deep-sea octacoral \\
\hline
\end{tabular}




\subsection{Figures}

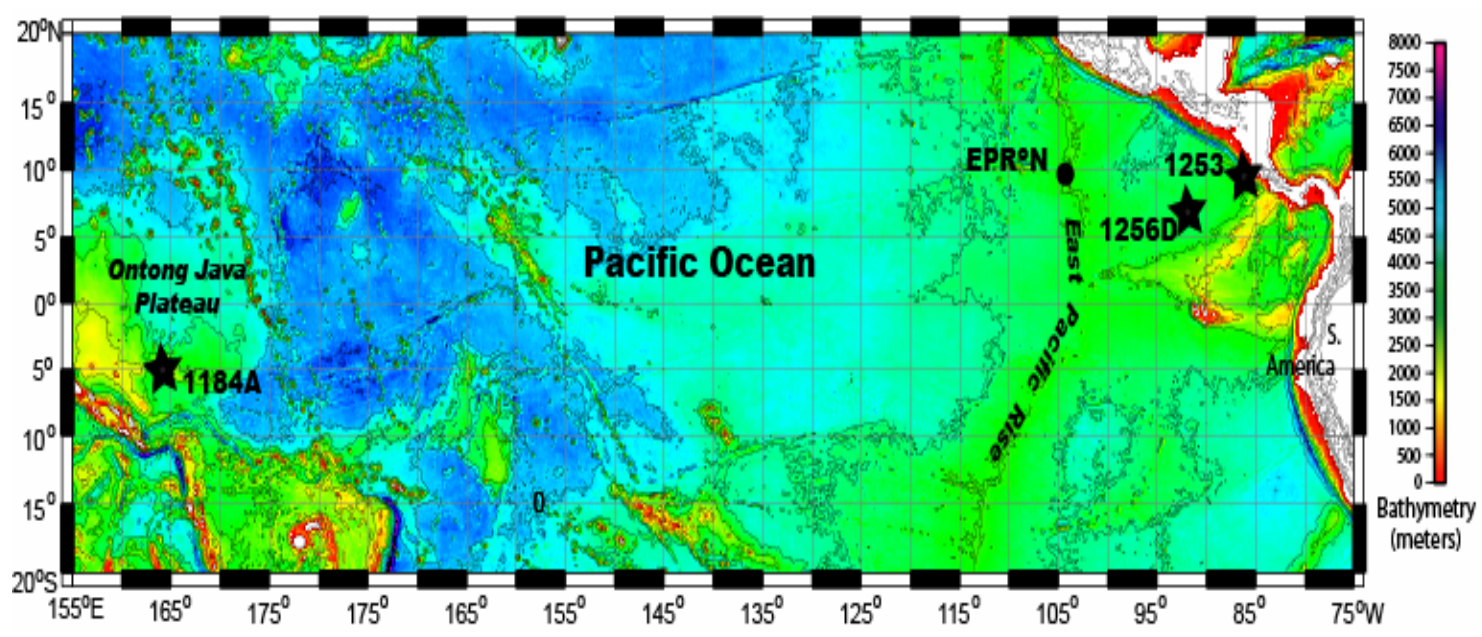

Figure 4.1. Bathymetric map of the Equatorial Pacific Ocean showing locations (ODP Holes 1184A, 1253, and 1256D) of samples used in this study. This map was produced using GeoMapApp. 


\subsection{References}

Alt, J.C., 1995. Subseafloor processes in mid-ocean ridge hydrothermal systems. In: S.E. Humphris, R.A. Zierneberg, L.S. Mullineaux and R.E. Thomson (Editors), Seafloor hydrothermal systems: physical, chemical, biological, and geological interactions. AGU Monograph. American Geophysical Union, Washington, DC, pp. 85-114.

Altschul, S.F. et al., 1997. Gapped BLAST and PSI-blast: a new generation of protein database search programs. Nucl. Acids Res., 25: 3389-3402.

Alvarez, A.J., Khanna, M., Toranzos, G.A. and Stotzky, G., 1998. Amplification of DNA bound on clay minerals. Molecular Ecology, 7: 775-778.

Bach, W. and Edwards, K.J., 2003. Iron and sulfide oxidation within the basaltic ocean crust: Implications for chemolithoautotrophic microbial biomass production. Geochim. Cosmochim. Acta, 67(20): 3871-3887.

Banerjee, N.R. and Muehlenbachs, K., 2003. Tuff life: Bioalteration in volcaniclastic rocks from the Ontong Java Plateau. Geochemistry Geophysics Geosystems, 4: Art. No. 1037(art. no. 1037).

Biddle, J.F. et al., 2006. Heterotrophic archaea dominate sedimentary subsurface ecosystems off Peru. Proc. Natl. Acad. Sci., 103(10): 3846-3851.

Brinkmeyer, R. et al., 2003. Diversity and structure of bacterial communities in Arctic versus Antarctic pack ice. Applied and Environmental Microbiology, 69(11): 6610-6619.

Chambers, L.M., Pringle, M.S. and Fitton, J.G., 2004. Phreatomagmatic eruptions on the Ontong Java Plateau: an Aptian ${ }^{40} \mathrm{Ar} /{ }^{39} \mathrm{Ar}$ age for volcaniclastic rocks at ODP Site 1184. In: J.G. Fitton, J.J. Mahoney, P.J. Wallace and A.D. Saunders (Editors), Origin and Evolution of the Ontong Java Plateau. Geological Society, London, Special Publications, 229.

Cowen, J.P. et al., 2003. Fluids from aging ocean crust that support microbial life. Science, 299(5603): 120-123.

DeLong, E.F. et al., 2006. Community genomics among stratified microbial assemblages in the ocean's interior. Science, 311: 496-503.

D'Hondt, S. et al., 2004. Distributions of microbial activities in deep subseafloor sediments. Science, 306: 2216-2221.

Edwards, K.J., Bach, W. and McCollom, T.M., 2005. Geomicrobiology in oceanography: microbe-mineral interactions at and below the seafloor. TRENDS in Microbiology, 13(9).

Edwards, K.J., Bond, P.L., Gihring, T.M. and Banfield, J.F., 2000. An archaeal ironoxidizing extreme acidophile important in acid mine drainage. Science, 287(5459): 1796-1799.

Edwards, K.J., Rogers, D.R., Wirsen, C.O. and McCollom, T.M., 2003. Isolation and characterization of novel psychrophilic, neutrophilic, Fe-oxidizing, chemolithoautotrophic alpha- and gamma-proteobacteria from the deep sea. Appl. Environ. Microbiol., 69(5): 2906-2913. 
Emerson, D. and Moyer, C., 1997. Isolation and characterization of novel iron-oxidizing bacteria that grow at circumneutral pH. Appl. Environ. Microbiol., 63(12): 47844792.

Fisher, A.T. and Becker, K., 2000. Channelized fluid flow in oceanic crust reconciles heat-flow and permeability data. Nature, 403: 71-74.

Fisk, M.R., Giovannoni, S.J. and Thorseth, I.H., 1998. Alteration of Oceanic Volcanic Glass? Textural Evidence of Microbial Activity. Science, 281(978-980).

Fisk, M.R., Thorseth, I.H., Urbach, E. and Giovannoni, S.J., 2000. Investigation of microorganisms and DNA from subsurface thermal water and rock from the east flank of Juan de Fuca Ridge. Proceedings of the Ocean Drilling Program, Scientific Results, 168: 167-174.

Franchi, M., Ferris, J.P. and Gallori, E., 2003. Cations as mediators of the adsorption of nucleic acids on clay surfaces in prebiotic environments. Origins of Life and Evolution of the Biosphere, 33: 1-16.

Furnes, H. and Staudigel, H., 1999. Biological mediation in ocean crust alteration: how deep is the deep biosphere. Earth and Planetary Science Letters, 166: 97-103.

Furnes, H. et al., 2001. Bioalteration of basaltic glass in the oceanic crust. Geochemistry, Geophysics, Geosystems, 2: Art. No. 2000GC000150.

Giovannoni, S.J., Fisk, M.R., Mullins, T.D. and Furnes, H., 1996. Genetic evidence for endolithic microbial life colonizing basaltic glass/sea water interfaces.

Proceedings of the Ocean Drilling Program, Scientific Results, 148: 207-213.

Huber, J., Butterfield, D. and Baross, J., 2003. Bacterial diversity in a subseafloor habitat following a deep-sea volcanic eruption. FEMS Microbiol. Ecol., 43(3): 393-409.

Huber, J.A., Butterfield, D.A. and Baross, J.A., 2002. Temporal Changes in Archaeal Diversity and Chemistry in a Mid-Ocean Ridge Subseafloor Habitat. Appl. Environ. Microbiol., 68(4): 1585-1594.

Huber, J.A., Johnson, H.P., Butterfield, D.A. and Baross, J.A., 2006. Microbial life in ridge flank crustal fluids. Appl. Environ. Microbiol., 8(1): 88-99.

Johnson, H.P. and Semyan, S.W., 1994. Age variation in the physical properties of oceanic basalts: Implications for crustal formation and evolution. J. Geophys. Res., 99: 3123-3134.

Khan, S.T., Horiba, Y., Yamamoto, M. and Hiraishi, A., 2002. Members of the family Comamonadaceae as primary poly (3-hydroxybutyrate-co-3-hydroxyvalerate)degrading denitrifiers in activated sludge as revealed by a polyphasic approach. Appl. Environ. Microbiol., 68(7): 3206-3214.

Kormas, K.A., Smith, D.C., Edgcomb, V. and Teske, A., 2003. Molecular analysis of deep subsurface microbial communities in Nankai Trough sediments (ODP Leg 190, Site 1176). FEMS Microbiology Ecology, 45: 115-125.

Lever, M.A. et al., 2006. Trends in basalt and sediment core contamination during IODP Expedition 301. Geomicrobiology Journal, 23: 517-530.

Lin, L.H. et al., 2006. Long-term sustainability of a high-energy, low-diversity crustal biome. Science, 314: 479-482.

Lysnes, K. et al., 2004a. Microbial community diversity in seafloor basalt from the Arctic spreading ridges. FEMS Microbiology Ecology, 50: 213-230. 
Lysnes, K., Torsvik, T., Thorseth, I.H. and Pedersen, R.B., 2004b. Microbial populations in ocean floor basalt: Results from ODP Leg 187. In: R.B. Pedersen, D.M.

Christie and D.J. Miller (Editors), Proceedings of the Ocean Drilling Program, Scientific Results.

Mahoney, J.J., Fitton, J.G., Wallace, P.J. and etal., 2001. Proc. ODP, Init. Repts., 192 [Online]. Available from World Wide Web: http://wwwodp.tamu.edu/publications/192 IR/192ir.htm.

Morris, J.D., Villinger, H.W., Klaus, A. and etal., 2003. Proc. ODP, Init. Repts., 205: College Station, TX (Ocean Drilling Program).

Nercessian, O., Fouquet, Y., Pierre, C., Prieur, D. and Jeanthon, C., 2005. Diversity of Bacteria and Archaea associated with a carbonate-rich metalliferous sediment samlple from the Rainbow vent field on the Mid-Atlantic Ridge. Environmental Microbiology, 7(5): 698-714.

Noonan, J.P. et al., 2005. Genomic sequencing of pleistocene cave bears. Science, 309: 597-600.

Reysenbach, A.L., Holm, N.G., Hershberger, K., Prieur, D. and Jeanthon, C., 1998. In search of a subsurface biosphere at a slow-spreading ridge. Proceedings of the Ocean Drilling Program, Scientific Results, 158: 355-360.

Rogers, D.R., Santelli, C.M. and Edwards, K.J., 2003. Geomicrobiology of deep-sea deposits: estimating community diversity from low-temperature seafloor rocks and minerals. Geobiology, 1: 109-117.

Shipboard_Scientific_Party, 2004. Costa Rica hydrogeology. IODP Prel. Rept., 301T. doi:10:2204/iodp.pr.301T.2004.

Shock, E.L. and Holland, M.E., 2004. Geochemical energy sources that support the subsurface biosphere. In: W.S.D. Wilcock, E.F. DeLong, D.S. Kelley, J.A. Baross and S.C. Cary (Editors), The Subseafloor Biosphere at Mid-Ocean Ridges. American Geophsyical Union.

Silver, E.A., 2001. Leg 170: synthesis of fluid-structural relationships of the Pacific margin of Costa Rica. In: E.A. Silver, G. Kimura, P. Blum and T.H. Shipley (Editors), Proceedings of the Ocean Drilling Program, Scientific Results, pp. 111.

Skidmore, M., Anderson, S.P., Sharp, M., Foght, J. and Lanoil, B.D., 2005. Comparison of microbial community compositions of two subglacial environments reveals a possible role for microbes in chemical weathering processes. Applied and Environmental Microbiology, 71(11): 6986-6997.

Smith, D.C. et al., 2000a. Methods for quantifying potential microbial contamination during deep ocean coring. ODP Technical Note, 28: 1-19.

Smith, D.C. et al., 2000b. Tracer-Based Estimates of Drilling-Induced Microbial Contamination of Deep Sea Crust. Geomicrobiology Journal, 17: 207-219.

Staudigel, H., Chastain, R.A., Yayanos, A. and Bourcier, W., 1995. Biologically mediated dissolution of glass. Chemical Geology, 126: 147-154.

Staudigel, H., Hart, S.R. and Richardson, S.H., 1981. Alteration of the oceanic crust: Processes and timing. Earth Planet. Sci. Lett., 52(2): 311-327. 
Staudigel, H. et al., 2004. The ocean crust as a bioreactor. In: W.S.D. Wilcock, E.F. DeLong, D.S. Kelley, J.A. Baross and S.C. Cary (Editors), The Subseafloor Biosphere at Mid-Ocean Ridges. American Geophysical Union, pp. 325-341.

Stein, C.A., Stein, S. and Pelayo, A., 1995. Heat flow and hydrothermal circulation. In: S.E. Humphris, R.A. Zierenberg, L.S. Mullineaux and R.E. Thomson (Editors), Seafloor Hydrothermal Systems: Physical, Chemical, Biological, and Geological Interactions. American Geophysical Union, pp. 425-445.

Thorseth, I.H., Furnes, H. and Heldal, M., 1992. The importance of microbiological activity in the alteration of natural basaltic glass. Geochim. Cosmochim. Acta, 56: 845-850.

Thorseth, I.H., Furnes, H. and Tumyr, O., 1995. Textural and chemical effects of bacterial activity on basaltic glass: an experimental approach. Chemical Geology, 119: 139-160.

Thorseth, I.H., Pedersen, R.B. and Christie, D.M., 2003. Microbial alteration of 0-30-Ma seafloor and sub-seafloor basaltic glasses from the Australian Antarctic Discordance. Earth and Planetary Science Letters, 215: 237-247.

Torsvik, T., Hurnes, H., Muehlenbachs, K., Thorseth, I.H. and Tumyr, O., 1998. Evidence for microbial activity at the glass-alteration interface in oceanic basalts. Earth and Planetary Science Letters, 162: 165-176.

Webster, G., Newberry, C.J., Fry, J.C. and Weightman, A.J., 2003. Assessment of bacterial community structure in the deep sub-seafloor biosphere by $16 \mathrm{~S}$ rDNAbased techniques: a cautionary tale. Journal of Microbiological Methods, 55: 155164.

Wilson, D.S., 1996. Fastest known spreading on the Miocene Cocos-Pacific plate boundary. Geophys. Res. Lett., 23: 3003-3006.

Wilson, D.S., Teagle, D.A.H., Acton, G.D. and etal., 2003. Proc. ODP, Init. Repts., 206. College Station, TX (Ocean Drilling Program).

Wilson, D.S. et al., 2006. Drilling to gabbro in intact ocean crust. Science, 312: 10161020. 


\section{Chapter 5. Characterization of Low-Temperature Alteration Products Associated with Young Basaltic Glasses from the East Pacific Rise at $9^{\circ} \mathrm{N}$ : Results of Synchrotron-based $\mu \mathrm{XRF}$ and $\mu \mathrm{XAS}$ Studies}

\subsection{Introduction}

The ocean crust, which is continually being produced at mid-ocean ridges, covers nearly $60 \%$ of the earth's surface. Large quantities of seawater circulate through the permeable, upper portion of the crust (Fisher and Becker, 2000) that is composed mainly of basaltic lava flows, and it is estimated that the entire volume of the ocean flows through the crust every one million years. As a consequence, basalt-seawater interactions at and below the seafloor largely control the geochemical cycles of many elements in both seawater and the ocean crust (Alt, 1995; Alt and Teagle, 1999; Bach et al., 2003; Elderfield and Schultz, 1996; Humphris and Thompson, 1978; Seyfried Jr. and Bischoff, 1979; Staudigel and Hart, 1983; Taylor and Lasaga, 1999; Wheat et al., 2003). At and below the seafloor, low-temperature, oxidative alteration reactions of reduced compounds (i.e., $\mathrm{FeO}$ and $\mathrm{MnO}$ ) in basaltic glass and minerals are thermodynamically favorable. These redox reactions represent a potential source of chemical energy for microbial growth (Bach and Edwards, 2003; Edwards et al., 2005). Indeed, 16S rRNA gene surveys indicate remarkable phylogenetic diversity and suggest likely metabolic diversity among communities of microorganisms in the volcanic lavas while quantitative gene analysis indicates high biomass (chapers 2 and 3 ). The role that the microorganisms play in the early stages of basalt weathering, however, is poorly constrained, principally 
because the initial processes and mechanisms controlling low-temperature alteration in the environment are not fully elucidated.

Laboratory experiments (Crovisier et al., 2003 and references therein; Daux et al., 1997; Gislason and Arnorsson, 1993; Gislason and Oelkers, 2000; Seyfried Jr. and Bischoff, 1979; Seyfried Jr. and Bischoff, 1981; Techer et al., 2001) have demonstrated that basaltic glass alteration and dissolution mechanisms are highly dependant on a number of factors: temperature, solution chemistry, glass geochemistry, alteration layer mineralogy, and extent of alteration. The study by Crovisier et al. (1987) shows that at low temperatures (between 0 and $60^{\circ} \mathrm{C}$ ), the long-term, steady-state dissolution of basaltic glass in a seawater medium under laboratory conditions proceeds through congruent dissolution of the glass matrix with subsequent precipitation of secondary minerals at the glass/seawater interface producing an alteration rind. In addition to these abiological factors, laboratory experiments with chemolithoautotrophic bacteria have demonstrated that the dissolution of basaltic rock (Daughney et al., 2004) and glass (Edwards et al., 2004) are greatly enhanced (increased release of Fe, Mn, and Si relative to abiotic controls) in the presence of these microorganisms. Thus, biological processes could be included as a potential "factor" that controls basaltic glass alteration, however the mechanism by which these microorganisms contribute to alteration processes, and specifically which microorganisms contribute to alteration in-situ, has not yet been determined.

To establish the relevance of these lab-based experiments to the deep-sea and subsurface environments, numerous studies have characterized the geochemical 
composition (both elemental and isotopic) and mineralogy of naturally altered basaltic lavas. Most of these studies to date have employed electron-based techniques (e.g., electron microprobe, EMPA; transmission electron microscopy, TEM; analytical electron microscopy, AEM; and selected area electron diffraction, SAED) or bulk analysis, powder X-ray diffraction (XRD). These techniques have provided a wealth of information regarding the chemistry and mineralogy of basalt glass alteration products (e.g. Alt and Mata, 2000; Banerjee and Muehlenbachs, 2003; Giorgetti et al., 2001; Pichler et al., 1999; Porter et al., 2000; Schramm et al., 2005). However, these studies have primarily been limited to older, more pervasively altered basalts with highly crystallized secondary minerals. The concentration on examining older lavas may be a reflection of the difficulty in characterizing poorly ordered/poorly crystalline materials found in early basalt alteration stages using electron-based techniques (it is suggested that the alteration rind, commonly referred to as the palagonite layer, starts as an amorphous gel in the early stages of alteration which develops into clay type minerals with age).

Synchrotron-based X-ray techniques such as micro X-ray fluorescence ( $\mu \mathrm{SXRF})$, micro X-ray absorption spectroscopy ( $\mu \mathrm{SXAS})$, and micro X-ray diffraction ( $\mu \mathrm{SXRD}$ ) present a unique opportunity to analyze poorly crystalline minerals and their spatial relationship with respect to fresh, unaltered glass and other alteration products. These techniques may be less susceptible to difficulties associated with electron-based techniques. For example, electron beams have the potential to change or destroy the local atomic structure or chemical speciation (Manceau et al., 2002). A study by Alt et al. (2000) notes that the alteration layer, although appearing amorphous, is easily damaged 
by the electron beam. Another study (Crovisier et al., 1987) observed clay-like structures within the alteration layer that were destroyed under the beam prior to obtaining a SAED image. Additionally, electron diffraction is not sensitive to materials with a high number of structural defects or vacancies, or with short-range order (Manceau et al., 2002). Xrays, on the other hand, are typically less damaging to environmental samples prone to beam damage, and are more sensitive for discerning mineral structures that have a low degree of polymerization. The advantage of synchrotron-produced x-rays is that these $\mathrm{x}$ rays have a very high flux (for detection of elements at low concentrations), can be focused down to nanometer scales, and have a wide range of tunable energies (all elements have characteristic absorption energies). To date, not a single study has been published using synchrotron-based X-ray techniques to characterize naturally altered basalt glasses.

Here we present the results of spatially-resolved, micrometer scale analyses of low-temperature volcanic glass alteration using synchrotron based XRF and XAS. Our goal was to identify the phases produced in the early stages of basalt glass weathering in geologically young $(<20 \mathrm{kyrs})$, seafloor volcanic glasses. The samples analyzed were collected from the seafloor at the East Pacific Rise spreading center and ridge flanks at $9^{\circ} \mathrm{N}$. We used $\mu \mathrm{SXRF}$ in cross-section (depth profile) of weathered materials to map the chemical distribution and co-occurrence of some geochemically relevant elements, namely $\mathrm{Fe}, \mathrm{Mn}, \mathrm{Zn}, \mathrm{V}, \mathrm{Ti}, \mathrm{Ca}, \mathrm{Si}, \mathrm{Cr}, \mathrm{Ti}, \mathrm{Cu}$, and $\mathrm{K}$. At physically discrete and chemically different environments, we collected Fe K-edge micro-extended X-ray absorption fine structure ( $\mu \mathrm{EXAFS}$ ) spectra to identify the structure and speciation of the 
different Fe weathering products and mineral accumulations (e.g., Fe oxyhydroxides, Febearing clays, and Mn oxides). Our data show the presence of Fe-rich clays, similar to nontronite and ferruginous smectite, lining the surfaces of fresh glass in the alteration rind. Additionally, we observed isolated patches of Fe-oxides and oxyhydroxides and Mn-oxide minerals both filling the fractures and accumulating on the surfaces exposed directly to seawater. These spectroscopy data will allow us to better understand the processes involved in low-temperature alteration of basalt glasses, a process which could support a significant microbiological community.

\subsection{Materials and Methods}

\subsubsection{Sample collection and description}

Basaltic lava samples were collected in 2004 from the East Pacific Rise (EPR), $9^{\circ} \mathrm{N}$, using the DSV Alvin during research cruises AT11-7 and AT11-20 on the R/V Atlantis. These samples were collected from the seafloor up to $\sim 3 \mathrm{~km}$ away from the ridge axis. Based on the spreading rate of the EPR, these samples can be no older than 20 ky. Upon retrieval, samples were first sub-sampled for micro- and molecular biological analyses. The remaining portions were frozen at $-80^{\circ} \mathrm{C}$. Frozen samples for this study were thawed, rinsed in distilled water and dried at $\sim 60^{\circ} \mathrm{C}$ prior to embedding in epoxy to preserve the alteration layers on the lavas. Polished thin sections $(\sim 30 \mu \mathrm{m}$ thick $)$ were prepared from the epoxy embedded samples and used for X-ray analyses.

Three basalt samples representing different stages of glass alteration were selected

for analyses (descriptions given in Table 5.1). Sample AT11-7_3970_8 (Research 
cruise\#_Alvin dive\#_sample\#), referred to as sample EPR3970 for the remainder of the chapter, was collected from the a hackly sheet flow approximately $1.5 \mathrm{~km}$ east of the ridge axis in the $9^{\circ} 30^{\prime}$ area. The "top" of the sample (direction based on how the sample was sitting in situ) is composed entirely of glass that is coated with a thick $(\sim 2-5 \mathrm{~mm})$ ferromanganese crust (as observed in the thin-section light microscopy image in Fig. 5.4a) whereas one "side" directly exposed to seawater has dense crowns of Fe oxides surrounded by small particles of mixed Fe and Mn oxides (as observed in Fig. 5.6a). Sample AT11-20_4055_B1, referred to as sample EPR4055, was collected from $\sim 3 \mathrm{~km}$ west of the ridge axes at $9^{\circ} 30^{\prime} \mathrm{N}$. The upper portion of this aphyric, microcrystalline basalt sample from a lobate sheet flow has a $2 \mathrm{~mm}$ thick glass layer that is coated with a 2-3 mm thick ferromanganese crust. The origin of sample EPR7A, collected on research cruise AT11-7 is undocumented, although mineralogical observations suggest this sample was collected several kilometers from the ridge axis. This sample is composed entirely of glass, and is encrusted in a $2 \mathrm{~mm}$ thick layer of $\mathrm{Fe}$ and $\mathrm{Mn}$ oxides. As described, all three samples are encrusted in a thick layer of Mn oxides and Fe oxyhydroxides. It appears that these oxide crusts preserved the alteration layers on the basalt glass because these sections were the only ones in which the glass alteration rinds remained intact after the thin-sectioning process.

\subsubsection{Synchrotron-based micro X-ray fluorescence ( $\mu$-SRXF)}

The XRF measurements were performed on Beamline 10.3.2 at the Advanced Light Source (ALS), Lawrence Berkeley National Lab (Marcus et al., 2004a). Elemental 
maps of select areas on the thin-sections were collected using a monochromatic beam first with an incident energy of 13 or $10 \mathrm{keV}$ and again at $4 \mathrm{eV}$ (to map lower atomic mass elements, e.g., $\mathrm{Si}$ and $\mathrm{K}$ ). For all elements except $\mathrm{Fe}$, the $\mathrm{K}_{\alpha} \mathrm{X}$-ray emission lines were recorded. For mapping of $\mathrm{Fe}$, the $\mathrm{K}_{\beta}$ emission line was used because the $\mathrm{K}_{\alpha}$ line overlaps with $\mathrm{Mn} \mathrm{K}_{\beta}$. The beam size ranged from $5 \times 5 \mu \mathrm{m}$ to $16 \times 7 \mu \mathrm{m}$ and fluorescence was collected with a seven-element Fe solid-state detector. The step size was $20 \times 20 \mu \mathrm{m}$ for the coarse scale map of sample EPR3970_8 and $10 \times 10 \mu \mathrm{m}$ for coarse scale maps of samples EPR7A and EPR4055_B1. Fine scale maps were collected with a step size of 5 $\times 5 \mu \mathrm{m}$ or $3 \times 3 \mu \mathrm{m}$. Tricolor elemental maps using red, green, and blue as end-member colors were constructed by combing the low and high energy maps using software designed by the beamline scientist, Dr. Matthew Marcus.

\subsubsection{Micro X-ray absorption spectroscopy ( $\mu$ XAS)}

XAS, and more specifically EXAFS, is a powerful tool for determining the local structure of a specific element at the molecular scale, which can lead to the identification of the host mineral. For example, EXAFS spectroscopy produces information regarding the number of nearest neighbor atoms to a specific element ( $\mathrm{Fe}$ in this case) as well as the interatomic distances to those neighbors. EXAFS spectra are produced by exposing the sample material to X-rays of increasing energy (E) typically $40-1000 \mathrm{eV}$ above the threshold of an absorption edge (which is specific to a particular element). Photoelectrons are ejected from the absorbing atom which results in oscillations in the measured absorption coefficient due to backscattering of the wave from the nearest 
atomic neighbors. Normalized EXAFS spectra, $\chi(\mathrm{E})$, are converted from $\mathrm{E}$ space into $\mathrm{k}$ space $(\chi(\mathrm{k}))$, which is a function of the photoelectron wave vector $\left(\mathrm{k}\left(\AA^{-1}\right)\right)($ Teo, 1986).

Iron K-edge EXAFS measuresments were performed at Beamline 10.3.2 at the ALS using the same detector as that used for SXRF. Spectra were collected in fluorescence detection mode from discrete spots of interest identified using the elemental map. EXAFS spectra were collected from a range of -200 to $+500 \mathrm{eV}$ around the Fe Kedge. Numerous spectra were recorded from each point of interest, and beam damage was not evident. Reference spectra were graciously provided by the Scott Fendorf group (Department of Geological and Environmental Sciences, Stanford University) as raw, unnormalized data. These spectra have been described in a previous study (Hansel et al., 2003) and are further discussed below.

Both the experimental and reference Fe EXAFS spectra were processed and analyzed using the SIXPack Software (Webb, 2005). Processing of the fluorescence spectra background incorporated averaging of multiple spectra from the sample location, background subtraction, normalization, and spline fitting. The EXAFS spectra $(\chi(k))$ were then $k^{3}$-weighted, represented as $\chi(\mathrm{k}) \mathrm{k}^{3}$. A threshold energy $\left(\mathrm{E}_{0}\right)$ of $7123 \mathrm{keV}$ was used for all spectra. Principal component analysis (PCA) was performed with $k^{3}$ weighted spectra to determine the minimum number of components required to best describe the data, which is specified by the minimum of the indicator function. Target transformations were then performed on the same suite of spectrua used for PCA to evaluate the likelihood that a specific reference spectra exists as an end-member 
component, as indicated by the SPOIL parameter. SPOIL values less than 1.5 are considered excellent, 1.5-3 good, 3-4.5 fair and 4.5-6 poor (Malinowski, 1978).

The constraint on the number of components determined by PCA analysis was used as support for linear least-squares fitting of the experimental spectra with the reference spectra. The spectra were first analyzed using the 'Combination Fit' program designed by Matthew A. Marcus (ALS beamline scientist, Beamline 10.3.2) to determine the best several combinations of references to fit the experimental data. SIXPack was used for final least-squares fitting of the data using the combinations of components recommended by 'Combination Fit'. For fitting the data, energy was not allowed to float and the component sum was not forced to equal 1.0. Linear-least squares fitting was used to approximate the proportion of each component (reference) in each EXAFS spectrum. The precision of linear fitting is $\pm 5-10 \%$ (Manceau et al., 2002). The statistical goodness-of-fit between the experimental data and the spectral fit is provided by the reduced chi-squared $\left(\chi_{v}^{2}\right)$ parameter (Ravel, 2000). The best statistical fit has the lowest $\chi_{v}^{2}$ parameter (Newville, 2001).

\subsubsection{Reference materials}

A number of Fe-bearing minerals were used as model compounds for PCA and linear least-squares fitting of the data. As mentioned previously, all reference spectra, with the exception of the biogenic ferrihydrite and fresh basalt glass, were provided by the Dr. Scott Fendorf lab at Stanford University. The ferric iron oxide and iron hydroxide minerals (referred to collectively as Fe oxides) include several different 
ferrihydrite minerals (biogenic, $2 \mathrm{~L}$, and $6 \mathrm{~L}$ ). The chemical formula for ferrihydrite is suggested as $\mathrm{Fe}(\mathrm{OH})_{3} \bullet \mathrm{nH}_{2} \mathrm{O}$ (Hansel et al., 2003), although the structure of this mineral is not fully established and may depend on the environment or conditions in which it is formed. It is suggested that $6 \mathrm{~L}$-ferrihydrite has a similar structure, but higher degree of polymerization than 2L-ferrihydrite. However, a study by Manceau and Drits (1993) suggests that the $6 \mathrm{~L}$-ferrihydrite structure may be significantly different than the socalled 'protoferrihydrite' (2L-ferrihydrite). The 2L- and 6L- ferrihydrite reference materials (synthetic) and spectra used in this study are characterized in Hansel et al. (2003). What we refer to as "biogenic ferrihydrite" in this study was obtained from insitu incubation experiments of sulfide minerals at the seafloor (Edwards et al., 2003). As described in detail by Edwards et al., microscopic analyses complemented by molecular biological techniques strongly suggest that the ferrihydrite analyzed here was formed by chemolithoautotrophic Fe-oxidizing bacteria. The structure of this material is currently being determined using Fe EXAFS analyses by a post-doctoral investigator, Dr. Brandy Toner. Preliminary results indicate that the structure of the biogenic ferrihydrite exhibits lower structural ordering (shorter range order) by comparison to 2L-ferrihydrite, and also suggests a structural carbon component (Brandy Toner, unpublished data).

The reference spectra used in this study were collected from iron oxyhydroxide mineral references goethite $(\alpha-\mathrm{FeOOH})$ and lepidocrocite $(\gamma-\mathrm{FeOOH})$, which differ structurally by the arrangement of edge-sharing Fe-O octahedra (Schwertmann and Cornell, 2000). These materials were also synthesized and characterized by Hansel et al (2003), as were the three different green rust phases used as model reference spectra. 
Green rust phases $\left(\mathrm{Fe}_{6-\mathrm{x}}^{\mathrm{II}} \mathrm{Fe}^{\mathrm{III}}{ }_{\mathrm{x}}[\mathrm{OH}]_{12}\left[\left(\mathrm{SO}_{4}\right)_{\mathrm{x} / 2} \cdot 3 \mathrm{H}_{2} 0\right]\right)$ are hydrotalcite-group doublelayer hydrates with positively charged $\mathrm{Fe}(+2,+3)$ and $\mathrm{O}$ layers intercalated by chloride, sulfate, or carbonate anions (Bernal et al., 1959).

The phyllosilicate reference spectra were obtained from two different naturally occurring dioctahedral smectite-type clays, a ferruginous smectite and a nontronite clay. Both of these clays were initially obtained from the Source Clays Repository of the Clay Minerals Society, Columbia, Missouri. The ferruginous smectite SWa-1 is a nontronite clay, but the 'ferruginous smectite' name is typically applied to this specific source clay. The structural formula for SWa-1 is $\mathrm{Na}_{0.87}\left(\mathrm{Si}_{7.38} \mathrm{Al}_{0.62}\right)\left(\mathrm{Fe}^{\mathrm{III}}{ }_{2.67} \mathrm{Fe}^{\mathrm{II}}{ }_{0.01} \mathrm{Al}_{1.06} \mathrm{Mg}_{0.23}\right)$ $\left(\mathrm{O}_{20}(\mathrm{OH})_{4}\right)$ (Manceau et al., 2000). The nontronite clay is source clay NAu-1 with the structural formula $\mathrm{M}_{1.05}^{+}\left[\mathrm{Si}_{6.98} \mathrm{Al}_{1.02}\right]\left[\mathrm{Al}_{0.29} \mathrm{Fe}_{3.68} \mathrm{Mg}_{0.04}\right] \mathrm{O}_{20}(\mathrm{OH})_{4}$ (Keeling et al., 2000).

The basalt glass spectrum was taken from a spot on Sample EPR3970 used in this study. The location of the spectrum showed no evidence of alteration, thus represents the unaltered glass endmember.

\subsection{Results}

\subsubsection{Elemental mapping}

Three basaltic lavas collected from the seafloor at the East Pacific Rise were prepared into thin-sections and characterized by light microscopy and $\mu \mathrm{SXRF}$. Examination of the lava thin-sections using light microscopy reveals that all three of these lavas contain abundant fresh, unaltered glass. A thin layer $(\leq 100 \mu \mathrm{m}$ thick) of altered glass is present on the surfaces of these lavas and in fractures where seawater 
contacted the rock, as indicated by the yellow-orange "palagonite" rinds on the surfaces and filling the fractures (Figs. 1a, 2a, 4b, 5a, and 6a). These images also show black mineral precipitates coating the surfaces and partially filling many of the fractures.

The results of elemental mapping by $\mu \mathrm{SXRF}$ of the areas of interest in each sample are shown in Figs 1-7. All grayscale maps are shown in positive contrast, so that the bright areas represent high concentrations of the element of interest. These results are semi-quantitative so that the brightest areas indicate the highest concentrations). The tricolor (red, green, and blue) and two-color maps were produced to show the co-location of multiple elements in a single image. The brightness of a color represents concentration, as in the grayscale maps. However, the hue represents differences in concentration (Manceau et al., 2002). A color triangle is provided in the appendix of this chapter (Appendix 5.1) to visualize the color spectra produced from co-location of the end-member color-coded elements. For example, mixing of equal concentrations of red and green produce yellow, red and blue produces magenta, and mixing of green and blue produces cyan. The co-location of all three color-coded elements in equal concentrations produces white. The tricolor maps presented here are qualitative (i.e., uncalibrated), and are presented in false color. The red, green, and blue channels presented for each figure may be individually scaled to compare elements of high concentration (like Fe) with elements of low concentration (like V) (e.g., the element coded by red may have a concentration 100 times higher than the green coded element, but the scaling of the brightness of each color may render them visually equal). 
Elemental mapping results for Sample EPR7A are showing in Figures 5.1-3. A light microscope image is shown in Fig. 5.1a. As can be seen, some locations of the sample have been destroyed (primarily in the fresh to altered glass transition zones) by the thin-section preparation process. The tricolor map in Fig. 5.1b shows Mn in red, Si in green, and $\mathrm{K}$ in blue. From this map, we see that $\mathrm{Mn}$ is found primarily in the black oxide crust, $\mathrm{Si}$ is primarily in the fresh glass, and $\mathrm{K}$ is primarily in the alteration rind and in the fractures. The bright blue/turquoise areas prevalent in some of the fractures suggest that both $\mathrm{Si}$ and $\mathrm{K}$ are present in some of the material filling these fractures. The relatively pure red and green colors indicate that $\mathrm{Mn}$ and $\mathrm{Si}$ do not occur together in the same materials within the fluorescence limit of detection. The grayscale maps for individual elements $\mathrm{Fe}, \mathrm{Mn}, \mathrm{Ca}, \mathrm{Si}, \mathrm{Zn}$, and $\mathrm{K}$ are shown in Figs $1 \mathrm{c}-\mathrm{h}$. Here we see that Fe is present in high concentrations in all portions of the sample. $\mathrm{Mn}$ is highly concentrated in the outer oxide layer, but very low concentrations are present in the glass and alteration layer. $\mathrm{Ca}$ is enriched in the fresh glass, depleted in the alteration rind, and has low concentration in the oxide layer. $\mathrm{Si}$ is also enriched in the fresh glass, but only slightly depleted (relative to fresh glass) in the altered glass layer and more depleted in the oxide layer. $\mathrm{Zn}$ appears to correlate with $\mathrm{Fe}$, and $\mathrm{Zn}$ concentrations are highest in the oxide layer where Fe and $\mathrm{Mn}$ are both present. $\mathrm{K}$ is highly concentrated in the alteration rind, but is entirely absent in the fresh glass and very depleted in the oxide layer. The diffuse glow in the $\mathrm{K}$ signal in the lower right area of the section suggests that a small amount of $\mathrm{K}$ is present in the slide on which the section was prepared. 
Figure 5.2 presents a higher resolution close-up view of Region 1A (outlined in Fig. 5.1b) in Sample EPR7A. This area represents a fracture in the fresh glass that is filled with oxides and rimmed with a palagonitic layer (Fig. 5.2a). The tricolor map (Fig. 5.2b) with Mn coded as red, Fe coded as green, and $\mathrm{K}$ as blue shows that $\mathrm{Fe}$ and $\mathrm{Mn}$ coexist in most of the highly concentrated oxide layer (indicated by the orange hue), but a small portion of this layer appears highly depleted in Mn (indicated by the pure green color). Grayscale maps of $\mathrm{Fe}$ and Mn (Figs. 2c and 2d) support this observation. The points of interest in Fig. 5.2b (labeled as 1 in the oxide layer and 20 in the glass alteration layer) show the locations of spots for Fe EXAFS analyses. It should be noted that the notation for the regions and points of interest for EXAFS throughout this study are not numbered sequentially, but rather follow the numbering scheme used during the collection of the data (not all data are presented). Elemental maps for $\mathrm{Ca}, \mathrm{Si}, \mathrm{K}$, and $\mathrm{Zn}$ (Figs. 2e-h) confirm observations made in Fig. 5.1, and maps of $\mathrm{Cr}$ and $\mathrm{Cu}$ (Figs. 2i and $2 \mathrm{j}$, respectively) show that these two elements are present in the oxide layer.

A more detailed, high resolution examination of Region 1B of sample EPR7A is provided in Fig. 5.3. This area shows a small portion of altered glass (upper left corner of each image) encrusted in a thick layer of oxides. The numbers on the tricolor map in Fig. 5.3a show the locations of spot analyses for Fe EXAFS. This map demonstrates a similar relationship for $\mathrm{Fe}$ and $\mathrm{Mn}$ in the oxide crust as in Fig. 5.2b, and supports our observation that $\mathrm{K}$ is not co-located in this layer. Figures $5.3 \mathrm{~b}, 5.3 \mathrm{f}$, and $5.3 \mathrm{~h}$ show that $\mathrm{Ti}$ and $\mathrm{V}$ also co-exist in the oxide layer. 
Sample EPR3970 is examined in Figs. 4-6. Three different regions were analyzed, two of which are outlined in the tricolor map, Fig. 5.4a, as Region 1 and Region 3. The third region, Region 6 , is several centimeters away from the area shown in Fig. 5.4a. A higher magnification image of EPR3970_Region1 is shown in Figure 5.4b. Here, a 'pillow' of oxides is sitting on altered glass. The lower right corner of this image is an area where the basaltic glass has been plucked out during sample preparation. The palagonitic layer (orange) differs slightly from that observed in sample EPR7A in that dark oxides are present both on top of the layer (exposed directly to seawater) and the bottom of the layer (closest to fresh, unaltered glass). Spots 1-5 (Fig. 5.4c) indicate the location of Fe EXAFS measurements. Tricolor maps (Figs. 4c and 4d) show the colocation of $\mathrm{Fe}, \mathrm{Zn}$, and $\mathrm{Mn}$ in the oxide pillow, but $\mathrm{Mn}$ appears to be excluded from the altered glass layer. Grayscale maps (Figs. 4e-4g) for $\mathrm{Fe}, \mathrm{Mn}$, and $\mathrm{Zn}$ confirm this observation.

Characterization of Region 3 of sample EPR3970 is shown in Figure 5.5. A light microscope image (Fig 5a) depicts fractures of various widths filled with altered glass and oxides. Examination of the tricolor map with $\mathrm{Mn}$ in red, $\mathrm{Fe}$ in green, and $\mathrm{K}$ in blue indicates that, unlike the oxide filled fractures in EPR7A, Mn is depleted in these areas. These dark particles are composed almost exclusively of Fe (for the elements measured here). Spots 1-5 (Figure 5.5b) indicate the location of Fe EXAFS measurements. The grayscale map for Mn (Fig. 5.5e) confirms that the Mn concentration is low in these Fe enriched particles. Both K (Fig. 5.5d) and Ca (Fig. 5.5f), however, show similar concentration patterns as in EPR7A. 
Region 6 of sample EP3970 was chosen specifically for analyses because of differences visualized in the oxide coating (Fig 6a) compared to all regions of interested examined to this point for both EPR3970 and EPR7A. In the light microscopy images of Region 6, it is apparent that the color of the oxides is not black, but rather a deep red hue. A higher magnification image (Fig. 5.6c) shows distinct morphologies of long sheath-like and stalk-like particles. These particular particle morphologies are similar to the particle morphologies produced by Fe-oxidizing bacteria isolated from the Loihi Seamount (Emerson and Moyer, 2002). Elemental mapping suggests that these particles are different geochemically from oxide coating examined previously in that the $\mathrm{Fe}$ and $\mathrm{Mn}$ do not seem to coexist (as seen in the both tricolor maps $6 \mathrm{~b}$ and $6 \mathrm{~d}$ ). Mn does however appear to be present in the basaltic glass. The numbers in Fig. 5.4b indicate the locations of Fe EXAFS analyses. The grayscale map of $\mathrm{Cr}$ (Fig. 5.6g) indicates that this element is enriched in the alteration rind. The Zn (Fig. 5.6h) and V (Fig. 5.6j) concentrations seem to follow that of Fe.

Elemental mapping of sample EPR4055 is shown in Figure 5.7. The numbers 1-3 in Fig. 5.7a indicate the 3 spot analyses for Fe EXAFS (spot 1 in the alteration rind, spot 2 in the oxide coatings, and 3 in a small fracture). The tricolor map and grayscale maps show that the oxide layer is enriched in both Fe (Fig. 5.7c) and Mn (Fig. 5.7d), as well as Cr (Fig. 5.7e) and V (Fig. 5.7i). Both Ca (Fig. 5.7f) and Ti (Fig. 5.7g) appear to be located only in the fresh glass, whereas Si (Fig. 5.7h) is most concentrated in the fresh glass, somewhat depleted in the alteration rind, and more depleted in the oxide coatings. 


\subsubsection{Fe EXAFS Analysis}

Fe K-edge EXAFS spectra were collected for 23 different points of interest based on the results of elemental mapping of the three different samples. The experimental spectra are compared to spectra for materials with known mineralogical structure and composition. These fully processed, $\mathrm{k}^{3}$-weighted reference spectra are shown in Fig. 5.8 for the range of 2-11 $\AA^{-1}$. The differences in phase, amplitude, and structure of the spectra are attributed to structure differences for the different minerals. The gray shaded areas (indicator regions) act as a guide to highlight some of the spectral features used distinguishing many of the $\mathrm{Fe}$ oxides and oxyhydroxides. For example, amplitude differences in the peak around $7.5 \AA^{-1}$ (the second indicator region) between $2 \mathrm{~L}$ ferrihydrite and goethite is likely the result of a lower Fe coordination number (fewer FeFe bonds) for ferrihydrite representing differences in mineralogical structure. Although basaltic glass is considered amorphous, Fe-O bonds are detected by Fe EXAFS and give rise to the oscillations observed.

The experimental Fe EXAFS spectra are shown in Figures 5.9-5.11. These spectra are separated into three different groups based on visible spectral similarities and differences compared with the references, rather than being grouped by sample. Figure 5.9 shows a summary of all spectra that resemble the clay reference spectra (which is shown at the bottom of spectra 1-6). Not surprisingly, all of the spots from which these spectra were collected are located in the alteration rind on the surface or in veins with the exception of spectrum \#5 (EPR3970_Reg1_Spot5), which is an odd bean-shaped feature in the oxide crust. 
A number of spectra highly resembled the poorly crystalline Fe-oxides such as the biogenic ferrihydrite or 2L-ferrihydrite, and these spectra are depicted in Figure 5.10 with the biogenic ferrihydrite reference spectra. Most of the locations of these spectra are in the thick, black oxide crusts (based on thin-section visualization) or black mineral precipitates filling in the fractures. None of these spectra, however, were collected from EPR3970_Region6 (Fig. 5.6) where the red stalk-like mineral precipitates were observed. The experimental spectra from these red minerals are are grouped together in Fig. 5.11, which shows spectra that resemble the more crystalline Fe oxides (e.g. 2L-ferrihydrite) or spectra that have features suggesting they may be a mixture of different minerals.

It is apparent upon visual inspection that some of the experimental Fe K-edge EXAFS spectra closely resemble certain reference spectra, whereas some experimental spectra are slightly different and may resemble mixtures of different spectra. In order to identify the number of different spectral components in the multicomponent dataset, principal component analysis (PCA) was performed using the experimental Fe EXAFS spectra. PCA determines the number of statistically significant spectral components or signatures supported by the dataset (Manceau et al., 2002). PCA performed using all experimental spectra (\#s 1-23) suggests that 3 different spectral components are present in the entire data set. However, only two components are required to explain all $\mathrm{Fe}$ oxide-like and intermediate spectra (\#s 7-23), indicating that the clay-like spectra (\#s 1-6) are mathematically accounted for by a single spectral component.

PCA is informative for objectively calculating the number of spectral components best supported by a dataset. These spectral components however, do not correspond 
directly to specific Fe-bearing minerals. For example, the first and most statistically significant component of this dataset is composed of the spectral features common to most of the Fe EXAFS spectra (most likely the spectral features of Fe(III)-O octahedra). PCA results may be interpreted as providing the number of significantly different mineral structures represented in a spectral dataset. However, if a specific spectral feature (representing a specific mineral) is present in all spectra, then this component may not be counted separately in the analysis, leaving one with a "missing" mineral. As with all statistical analyses of complex data, the information extracted from the analyses must be interpreted with the known chemistry of the system in mind.

Target transformation, a complementary technique to PCA, was employed to evaluate which reference spectra from the reference library, in this case minerals, are the best candidates for describing the experimental data (Manceau et al., 2002). Target transformations were performed using all the reference spectra presented in Figure 5.8 to the various spectral groupings as indicated for PCA. Results of target transformations are shown in Table 5.2. The three most suitable reference spectra for the entire spectral dataset, based on the SPOIL values, are ferruginous smectite, nontronite, and 6Lferrihydrite; however, only the two phyllosilicates rank as 'excellent' representatives for the dataset, and all of the reference spectra produce 'good' SPOIL values. Although the two clay references have the lowest numbers, it does not necessarily mean that both of these clays are required to explain the data. It is likely that only a single clay reference is necessary to fit the data (especially since the spectra are structurally very similar). The two best reference spectra to describe the experimental spectra 7-23 (all spectra except 
the clay-like spectra) are those collected from 6L-ferrihydrite and goethite, although all spectra, with the exception of basalt glass, produced 'good' SPOIL values.

It has been previously identified that EXAFS spectra can be treated as linear combinations of component spectra (Manceau et al., 1996), which provides another way of determining the best references (or in this case, the Fe-bearing minerals) that fit the experimental spectra. Linear least-squares fitting is used to fit each individual spectrum to the reference library spectra, and the fitting can accommodate multiple component (multi-mineral) mixtures. Each experimental spectrum in this study has been fit using 1, 2 , or 3 components based on all combinations of references used in this library. The best fits (the combinations with the lowest $\chi_{v}{ }^{2}$ value) for each spectrum are shown in Figures $5.12-5.21$.

Linear least-squares fitting of all clay-like spectra (Figure 5.11) suggests that spectra 2-6 are all statistically indistinguishable (fitting produced similar results for the best mixture of components and the relative proportion of each; data not shown), and that spectrum \#1 is slightly different. Spectrum \#1, sample EPR7A_Reg1B_Spot3, is collected from the center of an area highly enriched in $\mathrm{K}$ that looks like highly altered basalt glass. Figure 5.12A shows the fit for this spectrum using 1 component (a), 2 components (b), and 3 components (c). PCA suggests that only a single component is necessary to fit the clay-like spectra. However, a slight shift in phase to higher energies (i.e., a higher $\mathrm{k}$ value) is observed for the fitted data (dotted) relative to the experimental data (solid line) for a one component fit. The addition of components does not significantly improve the spectral fit. Figure 5.12B shows that ferruginous smectite 
produces the best single component fit, but the component only sums to 0.82 (a perfect fit would sum to 1.0 ) and the reduced $\chi^{2}$ value of 1.08 is quite high, suggesting a relatively poor fit. The best two and three component fits suggest that green rust-SO $\mathrm{SO}_{4}$ and lepidocrocite, respectively, each improve the fit (the sum is closer to 1.0 and the reduced $\chi^{2}$ is lower). However, visual inspection of the fits to the data shows that although the phase shift is not as large, the spectra feature on the shoulder of the first oscillation at approximately $5 \AA^{-1}$ is not longer evident.

Spectrum \#4, which is collected from the glass alteration rind in EPR3970_Reg1_Spot4, is taken as a representative for spectra 2-6. The results of the linear least-squares fitting are shown in Figure 5.13. Only 1 and 2 component fits are shown, as a 3 component fit did not improve the fit based on the goodness-of-fit indicator. Like spectrum \#1, the best fit to the experimental data using a single component has a slight phase shift to higher $\mathrm{k}$ values for the entire spectrum, although the spectral features and amplitudes match fairly well. Fits for both nontronite and ferruginous smectite are shown to demonstrate the striking similarities between these spectra, which suggests that fingerprinting tools that we use in this study (PCA, linear least-squares fitting) may not be useful for distinguishing different types of clays. Figure 5.13B shows that this clay-like spectrum also identifies the ferruginous reference as the best one component fit, but nontronite with the addition of green rust-Cl provides the best two component fit. The improvement using two components for spectrum \#4 is similar to spectrum \#1, i.e., although the goodness of fit and phase shift is improved, the spectral feature at $5 \AA^{-1}$ again is no longer present. 
Linear least-squares fitting for the poorly-crystalline Fe oxide-like spectra in Fig. 5.10 fall into two separate subgroups. The first subgroup (spectra 7-12) is represented by spectrum \#9, an EXAFS spectra collected in the black oxide crust from sample EPR4055_Region1_Spot2. The best linear fit results are shown in Figure 5.14. As can be seen in part A of this figure, the fitted spectrum matches the experimental spectrum very well. A slight phase shift is detected at higher $\mathrm{k}$ values, but the data in this region are somewhat noisy. The reference spectra used for creating the best single component fit is biogenic ferrihydrite, and indeed the $\chi^{2}$ value is very good (low), as shown in Fig. 5.14B. The addition of a small proportion of ferruginous smectite improves the fit only slightly. The second subgroup determined for the spectra shown in Fig. 5.10 consists of spectra 13-15, from which spectrum \#14 is chosen as a representative. Spectrum \#14 was collected from an area that intersects a basalt glass alteration rind and a fracture in sample EPR3970_Region1_Spot1 that appears to be filled with altered glass and black oxide particles. The linear fit results for this spectrum are shown in Figure 5.15. The one component fit to the experimental data (a), shows a slight phase shift starting in the second oscillation (around $6 \AA^{-1}$ ) which worsens at higher $\mathrm{k}$ values. A two component fit (b) better matches the experimental spectra, but the addition of a third component does not improve the fit. Indeed, these observations are confirmed in Fig. 5.15B, which shows the sum of components and goodness-of-fit parameter using two components. Biogenic ferrihydrite contributes the most to the fit (0.9) and lepidocrocite contributes to a small proportion (0.14) of the two component fit. 
The remaining spectra (\#s 16-23 in Fig. 5.11) analyzed in this study were grouped because they resembled Fe-oxide spectra with a greater degree of structure (e.g., goethite or lepidocrocite) or they did not match any of the reference spectra based on visual inspection. Spectrum \# 16 was collected from an alteration rind within a fracture in EPR7A_Region1A_Spot20. The result of two component linear least-squares fitting is shown in Figure 5.16. One and three component fits were also performed with the data, but the one component fit did not yield a good fit and the three component fit did not improve the fit, thus the results are not shown here. The best two component fit is produced with biogenic ferrihydrite (largest contribution; 0.85 of 1.16) and nontronite (0.31) which closely resembles the experimental spectra (Fig. 5.16A), however the phase is shifted slightly to higher $\mathrm{k}$ values. The results of linear least-squares fitting suggest that this spectrum is unique with respect to the overall data set.

Spectrum \#17 also represents a unique spectrum within the data set, as shown in Figure 5.17. This spectrum was collected from EPR7A_Region1B_Spot4, a Fe-rich/ Mnpoor crust on altered basalt glass. The best one component (lepidocrocite) and two component (lepidocrocite and biogenic ferrihydrite) fits are shown for the experimental data. Although a two component fit is statistically a better fit (lower $\chi^{2}$ value shown in Fig. 5.17B), analysis of the plotted spectra in Fig. 5.17A shows that the two component fit does not reproduce the spectral structure observed at $\sim 4.5 \AA^{-1}$. Using three components, not shown, did not statistically improve the fit. In contrast, linear fitting of spectrum \#18 from EPR7A_Region1B_Spot5 (a region next to an oxide crust that looks like it entrained some altered glass chips) does not yield good results using either a one or 
two component fit (Figure 5.18). The best three component fit for the experimental spectrum uses contributions from 6L-ferrihydrite, lepidocrocite, and green rust-Cl (listed in order of decreasing proportion) to fit the data.

The best fits for both spectra \#19 and \#20, which were collected at two different spots within the oxide and palagonite filled fractures of EPR3970_Region3, are produced using two components. In Figure 5.19 (spectrum \#19), basaltic glass is the dominant component (1.01/1.24) and lepidocrocite accounts for a small $(0.24 / 1.24)$, but statistically relevant $(>10 \%)$, proportion of the fit. However, the two components producing the best fit for spectrum \#20 are biogenic ferrihydrite and ferruginous smectite (Figure 5.20). As observed in Fig. 5.20A, however, the fit falls short of simulating the data in the $\mathrm{k}$ range of $\sim 7-8$.

Linear least-squares fitting of the final three spectra (\#s 21-23) suggests that these spectra are statistically undifferentiated. Interestingly, these three spectra were all from EPR3970_Region6, an area with the red Fe-rich/Mn-poor, morphologically distinct particles resembling biogenically formed Fe oxides. Spectrum \#22 was chosen as a representative for this area, and the linear fit results are shown in Figure 5.21. Here, the best one (a) and two component (b) fits are compared to the experimental spectrum. Although a one component fit with 6L-ferrihydrite is statistically good, visualization of the fitted spectrum relative to the experimental spectrum (Fig. 5.21A) shows mismatches at two of the indicator regions (5 and $7.5 \AA^{-1}$ as highlighted in Fig. 5.8). Adding biogenic ferrihydrite as a second component (with an approximately equal proportion to 6Lferrihydrite) improves the goodness of fit. 


\subsection{Discussion}

Synchrotron-based $\mu \mathrm{XRF}$ and $\mu \mathrm{XAS}$ have been used in this study to characterize the geochemistry and mineralogy of basaltic glasses in the early stages of lowtemperature alteration. Three lava samples collected from the seafloor at the East Pacific Rise were analyzed in thin-section using these techniques to identify the products formed during basaltic glass alteration. Additionally, analysis of the oxide minerals coating the lavas provides insight as to the geochemical cycling of $\mathrm{Fe}$ and $\mathrm{Mn}$ at the seafloor as well as trace elements, such as $\mathrm{V}$, that may be affected by the accumulation of these crusts. The thick crusts that are visible on the surfaces of the lavas and continue into many of the fractures are not a result of basalt alteration, rather they are an accumulation of particle fallout and precipitation from distant hydrothermal activity. The Fe and Mn are oxidized in the buoyant plume, transported off-axis in the plume, and rain out onto the seafloor. Studies show that basaltic glass alteration also produces Fe oxides and oxydroxides, which may be some of the first products formed during the initial stages of alteration (Crovisier et al., 1987; Pichler et al., 1999; Schramm et al., 2005). Unfortunately, we are unable to distinguish between Fe oxides formed as alteration products from those precipitated as a result of hydrothermal activity. In this study, we assume that most of the oxides that encrust the surfaces and fill the fractures are a hydrothermal signature. However, Fe oxides observed specifically within an alteration rind or within palagonitefilled fractures could potentially result from basalt alteration processes. Regardless of the source, these oxides represent a significant portion (both spatially and geochemically) of 
each sample and the effect of these oxides crusts, which are prevalent in many areas along the seafloor, on the geochemisty of the seawater and the underlying basalt are not fully understood.

\subsubsection{Elemental Mapping by $\mu S X R F$}

Elemental mapping of the basaltic glasses using $\mu \mathrm{XRF}$ is an efficient method that provides an immediate assessment of the overall geochemistry of the sample. The elements mapped in this study were chosen because they potentially undergo redox transformations and concentration changes during low-temperature basalt glass alteration. Of course, there are some elements not shown here that are of interest for understanding seafloor alteration processes, particularly with respect to microbiological or other organic influences, such as P or C. Each synchrotron beamline is designed differently for specific uses, and the beamline used here (10.3.2 at the ALS) is not capable of analyzing elements below $\mathrm{Si}$ in atomic number. It should also be noted that $\mathrm{As}$ and Se were also mapped, but the concentration of these elements was below the detection limit of the instrument.

Although not a purely quantitative technique, XRF provides a clear visual picture of the changes in concentration in fresh glass relative to the altered glass layers on the surfaces and fractures, as well as the locations of one element with respect to another (as in the tricolor maps). Some of the clearly defined trends observed in XRF mapping of all three samples are: the depletion of $\mathrm{Ca}$ and $\mathrm{Si}$ and the enrichment of $\mathrm{K}$ in the altered glass

relative to fresh glass. These are trends typically observed in glass alteration studies (Alt, 1995; Alt and Mata, 2000; Schramm et al., 2005; Seyfried Jr. and Bischoff, 1979; 
Staudigel and Hart, 1983). In laboratory experiments with entirely fresh glass, the alkali and alkaline-earth metals are the first elements lost during alteration due to proton exchange, and the loss of Si (due to breaking of Si-O bonds) occurs after the other metals have been leached from the glass (Oelkers, 2001; Oelkers and Gislason, 2001). This initially incongruent dissolution mechanism occurs rapidly and stoichiometric (congruent) dissolution is attained within hours (Guy and Schott, 1989). The uptake of K from seawater is a trend commonly observed in basalts altered at low temperature and represents the fixation of this element in phyllosilicates, like celadonite and smectite (Alt, 1995; Staudigel and Hart, 1983; Staudigel et al., 1981).

The distribution of Mn, although highly enriched in the oxide crusts, was not discernable in the glass or the alteration products. However, Fe is easily detected in both the glass and the altered glass. It appears that $\mathrm{Fe}$ is depleted in all alteration rinds in which the altered glass can be clearly discerned from the hydrothermal oxides (Fig. 5.2c and Fig. 5.7c). These results contrast with the majority of studies which suggest that $\mathrm{Fe}$ passively increases in the palagonite layer relative to fresh glass through the precipitation of oxides and oxyhydroxides. Although a study by Staudigel and Hart (1983) show that this trend is non-systematic, which they attribute to varying redox conditions. An alteration layer depleted in Fe relative to fresh glass was also observed by Alt and Mata (2000), and they conclude that the glass was altered in reducing conditions with Fe(II) mobilized from the glass. Reducing conditions, particularly on the surfaces directly exposed to seawater, are not expected however in this study. The interpreted depletion needs to be confirmed with independent techniques such as electron microbe analysis. 
Based on the results of other studies, it was expected that the concentrations of $\mathrm{Zn}, \mathrm{Cu}$ and $\mathrm{Cr}$ in the altered glass would be depleted relative to fresh glass (Staudigel and Hart, 1983). In most cases, the difference in intensity of $\mathrm{Zn}$ between these two states was not discernable (Figs. 5.1g, 5.2h, and 5.6h). However, sample EPR3970 (Fig. 5.4g) shows a clear increase in $\mathrm{Zn}$ in the palagonite layer. The dark mineral precipitates observed in the light microscopy image (Fig. 5.4b) suggest the possible entrainment of $\mathrm{Fe}$ oxides in this area, however Fe EXAFS analyses shows the best fit to the spectrum collected in this area (Fig . 5.13) is a ferruginous smectite. To understand the geochemical trends observed in this region, subsequent analyses using X-ray diffraction and electron microprobe will be necessary. Differences in intensity of the $\mathrm{Cu}$ signal are not observed between fresh and altered glass in Fig. 5.2j, but Fig. 5.2i suggests a small depletion of $\mathrm{Cr}$ in the alteration layer. Unfortunately, elemental mapping of $\mathrm{Cr}$ using XRF can be misleading because the fluorescence emission lines for $\mathrm{Cr}$ and $\mathrm{Mn}$ are very close, causing the Mn signal to leak into the $\mathrm{Cr}$ channel. This is typically only a problem where the Mn signal is very high, but this is likely the cause of the observed $\mathrm{Cr}$ enrichments observed in Figs. 5.2i and 5.7e. On the other hand, data from Region 6 of sample EPR3970 show that the Cr signal (Fig. 5.6g) correlates strongly with the K signal (Fig. 5.6i), which suggests that $\mathrm{Cr}$ is enriched in the alteration layer. Because $\mathrm{Mn}$ is not detectable in the alteration layer and is quite low even in the oxide layer, the $\mathrm{Cr}$ enrichment is likely real. Its implication is unclear, but the $\mathrm{Cr}$ enrichment may be a consequence of incongruent dissolution of the basalt glass and residual enrichment of $\mathrm{Cr}$ oxide. 
The oxide crust appears to be composed primarily of a mixture of Fe and Mn oxides and oxyhydroxides (although the structure of the Mn oxides has yet to be determined). Elemental mapping of these crusts shows that the signals for $\mathrm{Ca}, \mathrm{K}$, and $\mathrm{Si}$ are detectable, although the concentrations of these elements relative to glass are significantly diminished. On the other hand, Zn concentrations appear to be highest in the oxide crust. Previous examination of a ferromanganese marine nodule (Marcus et al., 2004b) shows $\mathrm{Zn}$ from anthropogenic and natural sources is sorbed to the Mn oxide minerals but not the Fe oxide minerals. These results suggest that indeed $\mathrm{Zn}$ may be present primarily as a sorbed species, rather than a Zn-bearing mineral, in the samples analyzed in this study. Not surprisingly, $\mathrm{V}$ also appears to be enriched in the oxide crust. $\mathrm{V}$ exists as an oxyanion in seawater and has been demonstrated to be incorporated into iron oxyhydroxides during the entrainment of seawater into the hydrothermal plume where the oxides are forming (Edmonds and German, 2004). Although most elemental mapping of $\mathrm{V}$ in this study shows $\mathrm{V}$ enrichment in areas where the oxide crust has high concentrations of both Fe and $\mathrm{Mn}$ (making it difficult to discern if $\mathrm{V}$ is sorbed entirely to Fe or both Mn and Fe), the elemental maps of EPR3970_Region6 (Fig. 5.6) show that the V map corresponds directly with that for the Fe map, but not for the Mn map. The high resolution tricolor map in Fig. 5.6d supports this theory ( $\mathrm{Mn}$ in red remains a pure color, but the green $(\mathrm{Fe})$ and blue $(\mathrm{V})$ colors are blended). The structure of $\mathrm{V}$ incorporated into the Fe oxides has not been determined (Edmonds and German, 2004), but V EXAFS represents a potential tool for determining the structure. 


\subsubsection{Fe EXAFS}

Fe EXAFS was used to identify the Fe-bearing minerals produced in the basaltic glass alteration rind as well as in the hydrothermally derived oxides coating the surfaces and filling in portions of the fractures. PCA was first used to identify the number of minerals (components) required to explain the Fe EXAFS data set. For example, PCA of all experimental spectra suggests that 3 different minerals are present, and more specifically only one mineral is required to fit the clay-like spectra (\#s 1-6) and the rest of the spectra (\#s 7-23) can be explained by 2 different minerals. These last 17 spectra were not broken into smaller subgroups for PCA due to computational concerns (PCA performs more accurately with larger data sets). Target transformations suggest that essentially all of the different Fe-bearing reference minerals used in this study could potentially account for each of these different components, or minerals. Based on visual inspection of the $\mathrm{k}^{3}$-weighted Fe EXAFS reference spectra (Fig. 5.8), the target transformation results are not entirely surprising. With the exception of the three green rust references (which are not true Fe oxide minerals), all of the Fe oxide (inclusive of oxyhydroxides) spectra are structurally quite similar (major oscillations occur at the same

$\mathrm{k}$ values). With the exception of the asymmetry near $5 \AA^{-1}$, the clay spectra also resemble the oxide spectra. It is somewhat surprising that the green rust references (mixed Fe(II) and Fe(III) minerals) are also suggested as 'good' component matches, not only because the structures of the spectra are quite different (and do not seem to visually match the experimental spectra), but because these minerals are typically observed in environments where the oxidation of $\mathrm{Fe}$ is incomplete, such as in anaerobic soils (Hansel et al., 2003; 
Trolard et al., 1997). Based on the environmental conditions in which we presume alteration to occur and ferromanganese crusts to form (for the samples examined here), minerals formed primarily in highly oxic environments or where $\mathrm{Fe}(\mathrm{II})$ is rapidly oxidized are expected. The presence of green rust could imply micro-scale differences in conditions in which these minerals form. Additionally, green rust phases are of interest in environmental and geo-biological sciences because of their ability to act as templates for organic molecule formation and stabilization (Arrhenius, 2003).

Identification of the mineral(s) at each point of interest was achieved using linear least-squares fitting of the individual EXAFS spectrum with respect to the Fe mineral reference library. Therefore, all results shown are specific to the references shown in Fig. 5.8. While the reference library presented here covers a wide variety of Fe-bearing minerals typically observed in deep-sea environments, the list is not exhaustive. For example, oxydroxides such as akaganeite $(\beta-\mathrm{FeOOH})$ and feroxyhite $(\delta$ '-FeOOH $)$ are not included in this library, simply because we do not currently have these spectra available to us (although these minerals may soon be prepared for spectral analysis based on initial analysis which suggests that feroxyhite may be a good candidate for some of the spectra analyzed here). Additionally, we do not have a reference spectrum for celadonite, an Febearing micaceous mineral typically observed in altered basalts. We also note that hematite $\left(\alpha-\mathrm{Fe}_{2} \mathrm{O}_{3}\right)$ and magnetite $\left(\mathrm{Fe}_{3} \mathrm{O}_{4}\right)$ were included in all PCA target transformation analyses and linear fitting, but yielded very poor matches with the data set and the results are not shown. The high quality of linear fits observed (both visually and statistically) for 
almost all spectra, however, suggests that the minerals represented in the current reference library are in fact the correct components.

The initial goal of this study was to use synchrotron-based techniques to characterize the products formed during the early stages of low-temperature basaltic glass alteration. As discussed earlier, the identification of minerals formed specifically as a result of glass alteration are not easily discernable from those from unrelated sources, such as hydrothermal plume fallout and sorption. Based on microscopic analysis and XRF measurements of the 23 points of interest, we will treat the following spectra as glass alteration products: EPR7A_Reg1A_Spot20 (spectrum \#16), EPR7A_Reg1B_Spot3 (spectrum \#1), EPR7A_Reg1B_Spot3a (spectrum \#2), EPR3970_Reg1_Spot4 (spectrum \#4), EPR3970_Reg3_Spot3 (spectrum \#6), EPR3970_Reg3_Spot4 (spectrum \#19), and EPR4055_Reg1_Spot1 (spectrum \#3). Products filling in fractures, however, are particularly non-trivial to identify the source because the yellowish 'palagonite' has been only partially infiltrated with black opaque minerals that may or may not be related to glass alteration, especially the fracture in-fill of EPR3970_Reg3 that is enriched in K relative to fresh glass. Therefore, these other 3 spots could be regarded as "potential" alteration products.

One of the more recent studies to examine the evolution of palagonite layer formation (Stroncik and Schmincke, 2001) suggests that the glass alters in phases, and the first phase is the formation of an amorphous gel-like layer followed by a mix of amorphous and poorly-crystalline phases which ages to a highly crystalline layer rich in smectite-type minerals. The view of an amorphous layer formation during alteration has 
been observed by others (Alt, 1995; Staudigel and Hart, 1983). Other reports, however, suggest that distinct (although sometimes poorly crystalline) minerals occur immediately without the formation of an amorphous layer (e.g, Crovisier et al., 1987).

Linear least-squares fitting of all of the Fe EXAFS glass alteration spectra mentioned above clearly identifies distinct mineral(s) within the alteration layer, with the exception of spectra 16 and 19. Most of these spectra suggest that the ferruginous smectite reference provides the best one component fit (as recommended by PCA). Although spectrum \#1 was initially treated separately, the final result is still similar to spectra 2-6 assuming a one component fit, and the spectrum clearly shows structural features distinct from the basaltic glass spectrum, a truly amorphous material. The studies that report amorphous palagonite layers were performed on glass samples that were several (or many several) millions of years old. In this study, the ability to undoubtedly identify smectite clay minerals within young $(<20 \mathrm{kya})$ basalts suggests that either: 1. the glasses sampled here altered immediately to smectites without an amorphous transition. 2. the different alteration zones (defined by Stroncik and Schmincke (2001)) are too small to be resolved with this technique and averaging of the area results in a strong, indistinguishable signal by the smectites. 3. the intensity of the synchrotron-produced X-ray beam is able to resolve structures not typically seen using previous techniques.

The linear fit results of spectrum \#19 suggest that the materials filling in the fracture are a mix of basaltic glass and lepidocrocite. This interpretation of the spectrum could rule out the first hypothesis that an amorphous transition is not observed in these 
samples. Because the fracture is so small, however, it is possible that the beam was wide enough to collect information from both the fresh glass and the alteration products. Interestingly, the identification of lepidocrocite in this area suggests that smectites are not the only product formed during basalt glass alteration, however elemental mapping of this fracture identifies products enriched in $\mathrm{K}$ and depleted in Fe relative to the fresh glass. Clearly, this area may need further examination to elucidate the spectral components.

Linear fit results for spectrum \# 16 also shows the presence of Fe oxides (biogenic ferrihydrite) in addition to nontronite within the alteration layer, however the best fit for this spectrum still does not match the data very well (as seen in Fig. 5.16). This suggests the presence of a different, unidentified phase. Although not shown, a number of $\mathrm{Fe}$ EXAFS spectra were collected at very small intervals $(1 \mu \mathrm{m})$ across the palagonite layer observed in Fig. 5.2a. These spectra were not analyzed due to the high level of noise and because they all resembled the glass spectra. Spectrum \#16 was chosen for analysis because it did not specifically resemble the fresh glass spectra or the biogenic oxide spectra (identified as the mineral phase adjacent to palagonite layer as discussed below), which suggests an intermediate phase that is potentially amorphous. In hindsight, better care should have been taken to collect higher quality spectra suitable for further analysis all along the alteration front.

It is clear that interpretations 1 and 2 regarding the crystallinity of the alteration layer are difficult to either corroborate or refute. Testing of hypothesis \#3 may provide a way of helping clarify the structure of alteration layer. Examining samples of previously 
defined "amorphous, gel-like" alteration layers with the synchrotron-based techniques presented in this study could at least determine if the definition of amorphous is specific to the electron-based and laboratory X-ray-based techniques used in the other studies.

Linear least-squares fitting of Fe EXAFS spectra for the hydrothermally derived Fe oxides identifies several different mineral phases. Many of these spectra have very good one component fits with the biogenic ferrihydrite reference spectra, which would suggest that oxides produced in the plume are produced as a metabolic by-product of chemolithotrophic Fe oxidizing bacteria. An abiotic formation mechanism cannot be ruled out at this stage, however. Determination of the biogenic ferrihydrite structure suggests a strong association with C (Dr. Brandy Toner, personal communitication), so further analysis (shell-by-shell fitting) of the hydrothermal oxide crust Fe EXAFS spectra could eventually elucidate the formation mechanism (biologic or abiotic).

Surprisingly, none of the spectra resembling biogenic ferrihydrite were collected from the EPR3970_Region6 in which the oxide particle morphologies so closely resemble those produced by $\mathrm{Fe}$ oxidizing bacteria (including the morphologies of the oxides from which the biogenic ferrihydrite reference was collected). These sheath-like and twisted stalk morphologies (shown in Fig. 5.6c) have only been reproduced by biological processes in the laboratory. Linear fitting of spectra 21-23 (from EPR3970_Region 6) shows a two-component mixture of 6L-ferrihydrite and biogenic ferrihydrite. 6L-ferrihydrite, however, is not typically observed at the seafloor, and this spectrum is an excellent candidate for feroxyhite (Dr. Alain Manceau, personal communication) which can be verified with future analyses (discussed below). These 
results bring about a number of questions regarding the formation of these oxides: 1 . are the morphologies a good indicator for microbiological oxidation?, 2. does microbial activity produce minerals other than ferrihydrite?, and 3. could feroxyhite result from aging of biogenic ferrihydrite?. Question number 1 has been addressed above, and although an abiotic formation mechanism cannot be excluded, the strong resemblance to known biogenic morphologies supports the interpretation of a biologically induced formation mechanism. A search through the current literature does not explicitly link the formation of feroxyhite to microbial activity (Question 2), however feroxyhite is not observed in many environments except for in ferromanganese nodules (Carlson and Schwertmann, 1980; Manceau and Drits, 1993). One study did report that a small amount of feroxyhite was observed with biogenically formed ferrihydrite, but the mechanism of formation for the ferrihydrite was not speculated on (Banfield et al., 2000). Regarding question 3, studies examining the structure (Manceau and Drits, 1993) and occurrence (Carlson and Schwertmann, 1980) of feroxyite suggest that this mineral phase, like ferrihydrite, is a direct result of rapid $\mathrm{Fe}(\mathrm{II})$ oxidation and does not form from aging of other phases lacking long-range structural order. Additionally, a study by Schwertmann et al. (1968) shows that the presence of organic compounds inhibits the formation of Fe oxides with higher crystallinity, so the ageing of biogenic ferrihydrite is unlikely to result in the formation of feroxyhite.

One other $\mathrm{Fe}$ oxide phase, other than biogenic ferrihydrite and feroxyhite, accounts for some of the other spectral components identified by linear least-squares fitting. Lepidocrocite provides the best one-component fit for spectrum \#17, and 
accounts for one of the two components (with biogenic ferrihydrite) recovered in spectra 13-15. Lepidocrocite is typically found in environments where Fe(II) oxidation is slow due to low oxygen concentrations, and a biological mechanisms of formation has never been demonstrated. The observation of lepidocrocite with biogenic ferrihydrite is thus somewhat puzzling and needs to be confirmed with independent techniques, although a positive confirmation could be explained by the formation of micro-environments within the oxide crusts.

\subsection{Future Direction}

\subsubsection{Synchrotron-based micro $X$-ray diffraction ( $\mu$-SXRD)}

As was discussed earlier, EXAFS analysis is not the proper technique to distinguish the types of clays observed in the alteration layer on the basalt glass. X-ray diffraction is necessary to resolve the atomic structures of these minerals. Synchrotronbased XRD represents a powerful technique for determining the precise locations of different minerals and for studying poorly-crystalline minerals, multi-component mixtures, or minerals with small particle sizes (i.e. nanoparticles) due to the bright and focused nature of the X-rays relative to conventional laboratory based X-ray diffractometers (Manceau et al., 2002).

The proposal funding the work in this chapter supported analyses using two different end-stations at the ALS, Beamline 10.3.2 used for XRF and XAS analyses and Beamline 7.3.3 for XRD. Beamline 7.3.3 was decommissioned nearly a year ago and moved to a new end-station with a more focused (down to a $0.5 \times 0.5 \mu \mathrm{m}$ spot size) and 
powerful beam. Because of the transition, XRD analyses were not able to be completed. However, time has been allotted to finish this project starting this April (2007). These XRD measurements will allow us to distinguish the types of clays present in the alteration rind as well as confirm the interpretation of our XAS results with respect to the oxide minerals. Also, we will be able to identify any minerals present that were not accounted for in our reference library (especially celadonite) and confirm our interpretation of multi-component spectra.

\subsubsection{Electron microprobe analysis}

After completion of the XAS work, electron microprobe analysis (EMPA) will be performed at the University of Kiel, Germany to supplement the geochemical data provided by the synchrotron-based XRD, XAS and XRF analyses. EMPA will be used to quantify the elements from points of interest used for the previous analyses as well as provide additional elemental information, particularly of the low atomic weight elements ( $\mathrm{S}, \mathrm{P}, \mathrm{C}, \mathrm{N}$, etc.) that can not be detected by the XRF techniques used here. Additionally,

elements such as $\mathrm{Mg}, \mathrm{Si}$, and $\mathrm{Al}$ will be quantified to complement the data provided by XRD spot analyses. This approach is necessary to determine the atomic structure of clay minerals in the basaltic glass alteration rind.

\subsubsection{Manganese oxides}

As shown previously, thick layers of Fe and Mn oxides/oxyhydroxides are accumulating on the surfaces of the basalts. Although ferromanganese nodules and crust 
have been studied for many years (primarily geochemical composition), very little is known about the mechanisms of formation particularly with respect to the Mn oxide minerals. The abiotic oxidation rates of $\mathrm{Mn}(\mathrm{II})$ in deep seawater, although thermodynamically favorable, are very slow and it is suggested that microorganisms (which can catalyze $\mathrm{Mn}(\mathrm{II})$ oxidation) may potentially be playing a role in the formation of these minerals (Tebo et al., 2004). Soluble Mn(II) in the deep-sea is largely provided by hydrothermal venting, but it has been observed that $\mathrm{Mn}$ is also released from basaltic glass during low-temperature water-rock reactions (Staudigel and Hart, 1983). The Mn (primarily existing as $\mathrm{Mn}$ (II) in basaltic glass) could precipitate out at the basaltic surface, especially if Mn oxidizing bacteria are present. Indeed, phylogenetic evidence (Chapter 3) and culturing studies (Templeton et al., 2005) suggest that Mn oxidizing bacteria exist on young, basaltic glass surfaces.

XAS techniques provide a way of characterizing the mineralogical structure of Mn oxides and oxyhydroxides that are notoriously difficult to examine by nonsynchrotron techniques due to their typically short-range structure. Additionally, XAS has proven to be extremely efficient and consistent at determining the speciation of $\mathrm{Mn}$ $(+2,+3$, or +4$)$ oxides. To our knowledge, XAS techniques have only been used in a single study to examine ferromanganese minerals from a marine environment (Marcus et al., 2004b). The authors were able to characterize the Mn oxide mineralogy and structure within a ferromanganese nodule from the Baltic Sea. More spatially resolved, systematic studies to characterize the Mn mineralogy and speciation of $\mathrm{Mn}$ in deep-sea environments are necessary to elucidate their formation mechanisms. 
We have begun collecting Mn K-edge micro X-ray absorption near edge structure ( $\mu$ XANES) spectra of Mn-rich particles on basalt glass surfaces and in veins to characterize the structure and speciation of these oxide minerals. We are also interested in the potential for Mn oxides to contribute to basalt alteration process by oxidizing $\mathrm{Fe}$ (II) in the glass. Spatially resolving the speciation of $\mathrm{Mn}$ in the oxide layer (e.g. directly in contact with the glass or glass alteration layer vs. on top of the ferromanganese crust exposed to seawater) could possibly provide information necessary to assess this reaction.

\subsection{Summary}

Sychrotron-based $\mu \mathrm{XAS}$ and $\mu \mathrm{XRF}$ provides a powerful and unique way of examining the mineralogical phases associated with the early stages of basaltic glass alteration at the seafloor. Besides being non-destructive, the spatial resolution of these techniques allows for micro-scale characterization of large areas within the sample. In this study, we were able to clearly resolve several mineral phases (Fe-bearing clays and oxides) within the glass alteration layer, in the fractures, and within the hydrothermally derived $\mathrm{Fe}$ and $\mathrm{Mn}$ oxide crusts that infiltrate the samples. Our primary results suggest that smectite clays are both the initial and the dominant alteration product of basaltic glass alteration in this environment. An amorphous gel-layer typically cited as being the initial result of glass hydration is not explicitly observed, although this may be disputed with futher analysis. Additionally, Fe EXAFS analyses suggest a biological formation mechanism for much of the oxide crust, although abiotic formation in the presence of organic compounds cannot be ruled out at this time. Synchrotron-based $\mu$ XRD and 
EMPA analyses will complement the current observations to help resolve the formation mechanisms of these Fe-bearing phases. 


\subsection{Tables}

Table 5.1. Description of seafloor basalts sampled from the EPR used for XAS and XRF analyses.

\begin{tabular}{|c|c|c|c|c|c|}
\hline Sample $^{\mathrm{a}}$ & $\begin{array}{l}\text { Latitude } \\
\left({ }^{\circ} \mathrm{N}\right)\end{array}$ & $\begin{array}{l}\text { Longtitude } \\
\left({ }^{\circ} \mathrm{W}\right)\end{array}$ & $\begin{array}{l}\text { Depth } \\
\text { (m) }\end{array}$ & Field site description & Sample description \\
\hline $\begin{array}{l}\text { EPR3970 } \\
\text { AT11-7_3970_8) }\end{array}$ & $9^{\circ} 30^{\prime} 11^{\prime \prime}$ & $104^{\circ} 13^{\prime} 48^{\prime \prime}$ & 2667 & $\begin{array}{l}\text { Hackly flow between two } \\
\text { pillow mounds } \sim 0.5 \mathrm{~km} \\
\text { east of the } \mathrm{AST}^{\mathrm{c}}\end{array}$ & $\begin{array}{l}\text { Ferromanganese oxide crust } \\
\text { coating folded basalt with a } \\
\text { thin, altered glassy exterior }\end{array}$ \\
\hline $\begin{array}{l}\text { EPR4055 } \\
\text { (AT11-20_4055-B1) }\end{array}$ & $9^{\circ} 29^{\prime} 82^{\prime \prime}$ & $104^{\circ} 16^{\prime} 01^{\prime \prime}$ & 2665 & $\begin{array}{l}\sim 3 \mathrm{~km} \text { to west of AST- } \\
\text { basalt talus within } \\
\text { sediment }\end{array}$ & $\begin{array}{l}\text { Microcrystalline basalt with } \\
2 \mathrm{~mm} \text { thick glass rind, } \\
\text { slightly altered surface, thick } \\
\text { oxide coating }\end{array}$ \\
\hline $\begin{array}{l}\text { EPR7A } \\
\text { (AT11-7_7A) }\end{array}$ & $\mathrm{ND}^{\mathrm{b}}$ & ND & ND & ND & $\begin{array}{l}\text { Sample composed entirely of } \\
\text { glass, alteration on surface/ } \\
\text { fractures, } 2 \mathrm{~mm} \text { thick coating } \\
\text { of ferromanganese oxides }\end{array}$ \\
\hline
\end{tabular}

${ }^{a}$ Short sample name used throughout study and full sample name in parentheses below.

${ }^{b}$ Not determined

${ }^{c}$ Axial Summit Trough (AST) marking the mid-ocean ridge spreading axis along the EPR, often where new lava is erupted from. 
Table 5.2. Target transformation results for the experimental Fe EXAFS spectra with each reference spectra.

\begin{tabular}{lcc}
\hline & \multicolumn{2}{c}{ SPOIL function } \\
\cline { 2 - 3 } Reference Spectra & Spectra & Spectra \\
\hline basalt glass & 2.42 & $7-23$ \\
biogenic ferrihydrite & 2.40 & 3.28 \\
2L-Ferrihydrite & 2.52 & 2.83 \\
6L-Ferrihydrite & $1.59^{*}$ & $1.52^{*}$ \\
Goethite & 1.89 & $1.81^{*}$ \\
Lepidocrocite & 2.47 & 2.13 \\
Greenrust-CO & 2.19 & 2.96 \\
Greenrust-SO & 2.64 & 2.20 \\
Greenrust-Cl & 2.66 & 2.39 \\
Ferruginous smectite & $1.39^{*}$ & 2.24 \\
Nontronite & $1.37^{*}$ & 1.93 \\
\hline
\end{tabular}

${ }^{\#}$ SPOIL function values less than 1.5 are considered excellent, 1.5-3 good, 3-4.5 fair and 4.5-6 poor (Malinowski, 1978).

*Best reference spectra based on target transformations. The number of starred references per group of spectra represents the minimum number of components based on PCA results. 


\subsection{Figures}
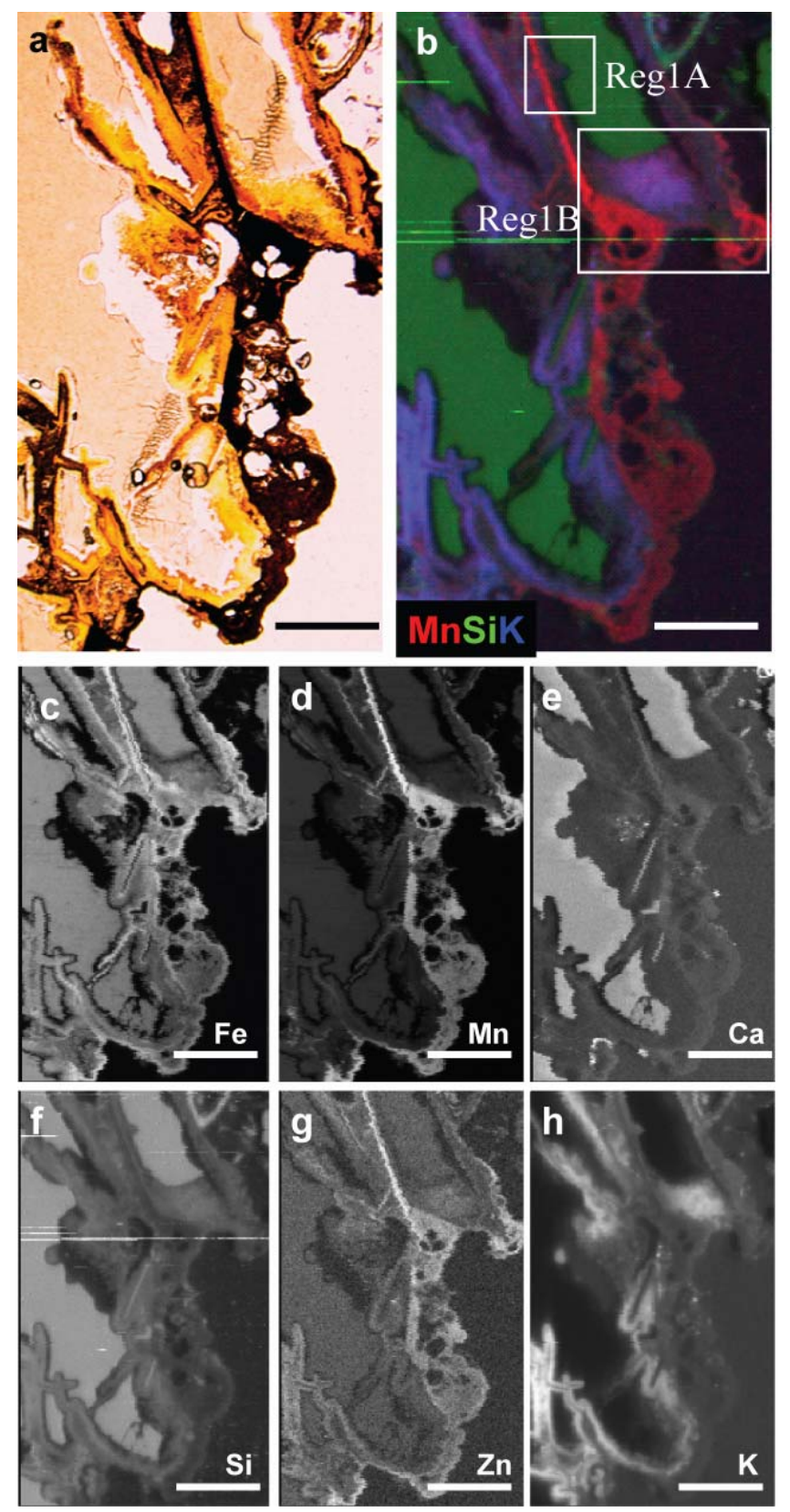

Figure 5.1. Characterization of EPR7A. (a) Light microscope image of the thin-section ( $\sim 30 \mu \mathrm{m}$ thick) showing the transition from fresh glass (beige color) through the alteration layer (yellow-orange rind) and into the Fe- and Mn-oxide coated surface (black). (b) $\mu \mathrm{SXRF}$ tricolor map with $\mathrm{Mn}$ coded as red, $\mathrm{Si}$ as green, and $\mathrm{K}$ as blue. The white boxes outline the regions (1A and 1B) used for more detailed analyses. (c-f) Grayscale maps for elements $\mathrm{Fe}, \mathrm{Mn}, \mathrm{Ca}, \mathrm{Si}, \mathrm{Zn}$, and $\mathrm{K}$ where bright colors indicate high concentrations. Scale bar is $500 \mu \mathrm{m}$ in all images. 

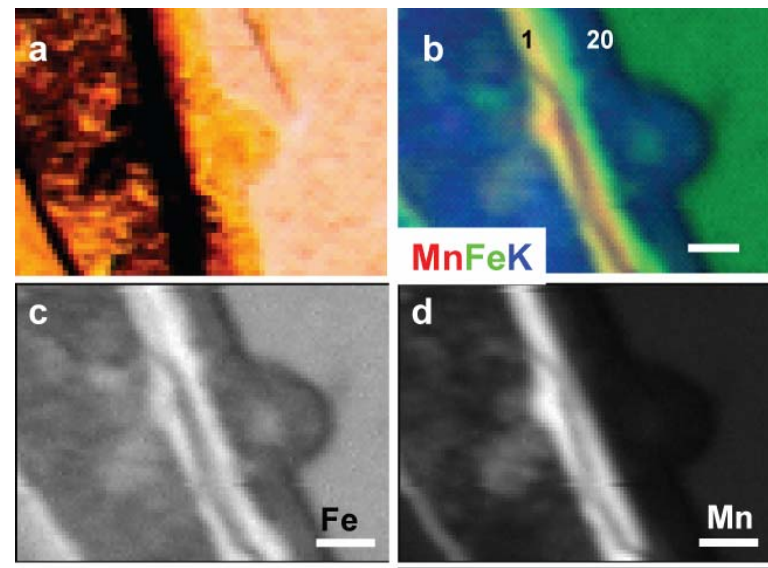

d
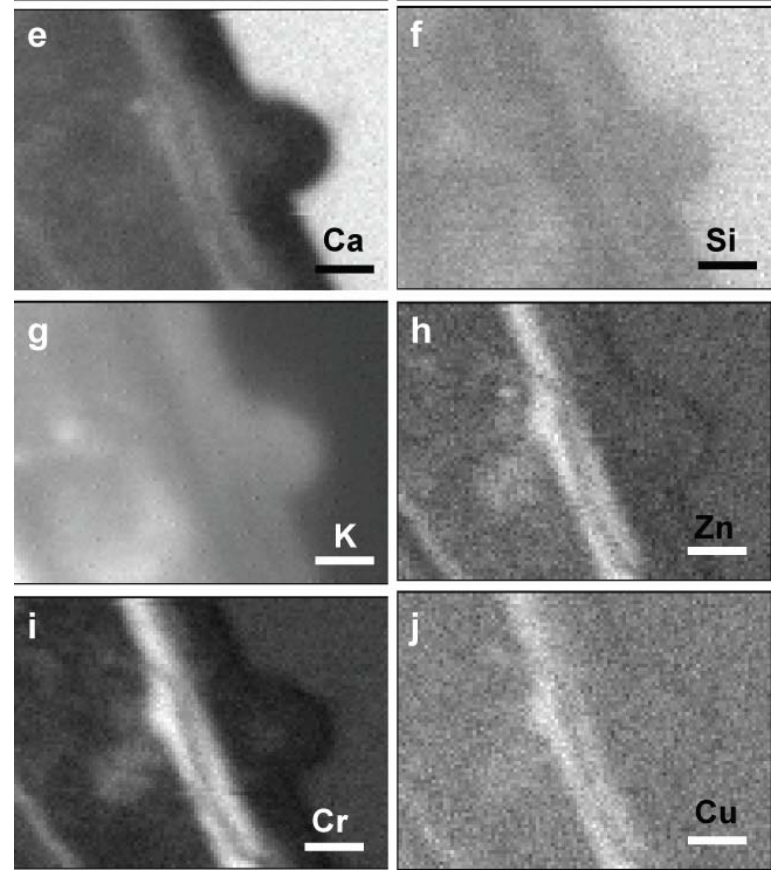

Figure 5.2. Elemental maps of EPR7A_Region1A. (a) Light microscope image of the fracture filled with altered glass and black mineralsprecipitates, through the alteration layer (yellow-orange rind), and into the fresh glass. (b) $\mu \mathrm{SXRF}$ tricolor map with Mn coded as red, Fe as green, and $\mathrm{K}$ as blue. The numbers ( 1 and 20) represent the locations of spot analyses used for EXAFS measurements. (c-j) Grayscale maps for elements Fe, $\mathrm{Mn}, \mathrm{Ca}, \mathrm{Si}, \mathrm{K}, \mathrm{Zn}, \mathrm{Cr}$, and $\mathrm{Cu}$ where bright colors indicate high concentrations. Scale bar is $50 \mu \mathrm{m}$ in all images. 

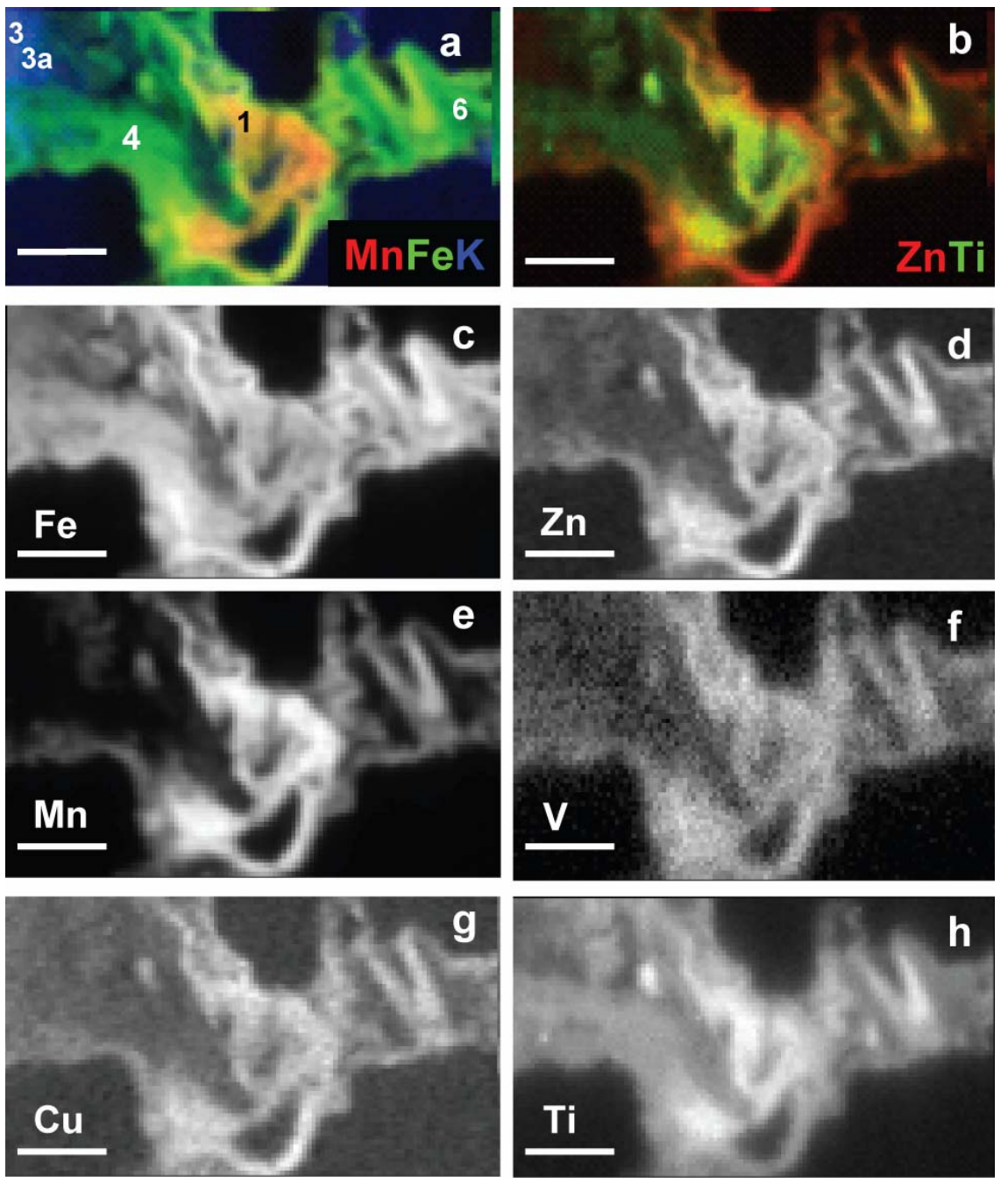

Figure 5.3. Elemental maps of EPR7A_Region1B (altered basalt glass coated with a thick layer of oxides). (a) $\mu \mathrm{SXRF}$ tricolor map with Mn coded as red, Fe as green, and $\mathrm{K}$ (an indication of the presence of clays) as blue. The numbers (1, 3, 3a, 4, and 6) represent the locations of spot analyses used for EXAFS measurements. (b) $\mu \mathrm{SXRF}$ tricolor map of the oxide crust with $\mathrm{Zn}$ coded as red and $\mathrm{Ti}$ as blue. (c-h) Grayscale maps for elements $\mathrm{Fe}, \mathrm{Zn}, \mathrm{Mn}, \mathrm{Ca}, \mathrm{V}, \mathrm{Cu}$, and $\mathrm{Ti}$ where bright colors indicate high concentrations. Scale bar is $100 \mu \mathrm{m}$ in all images. 

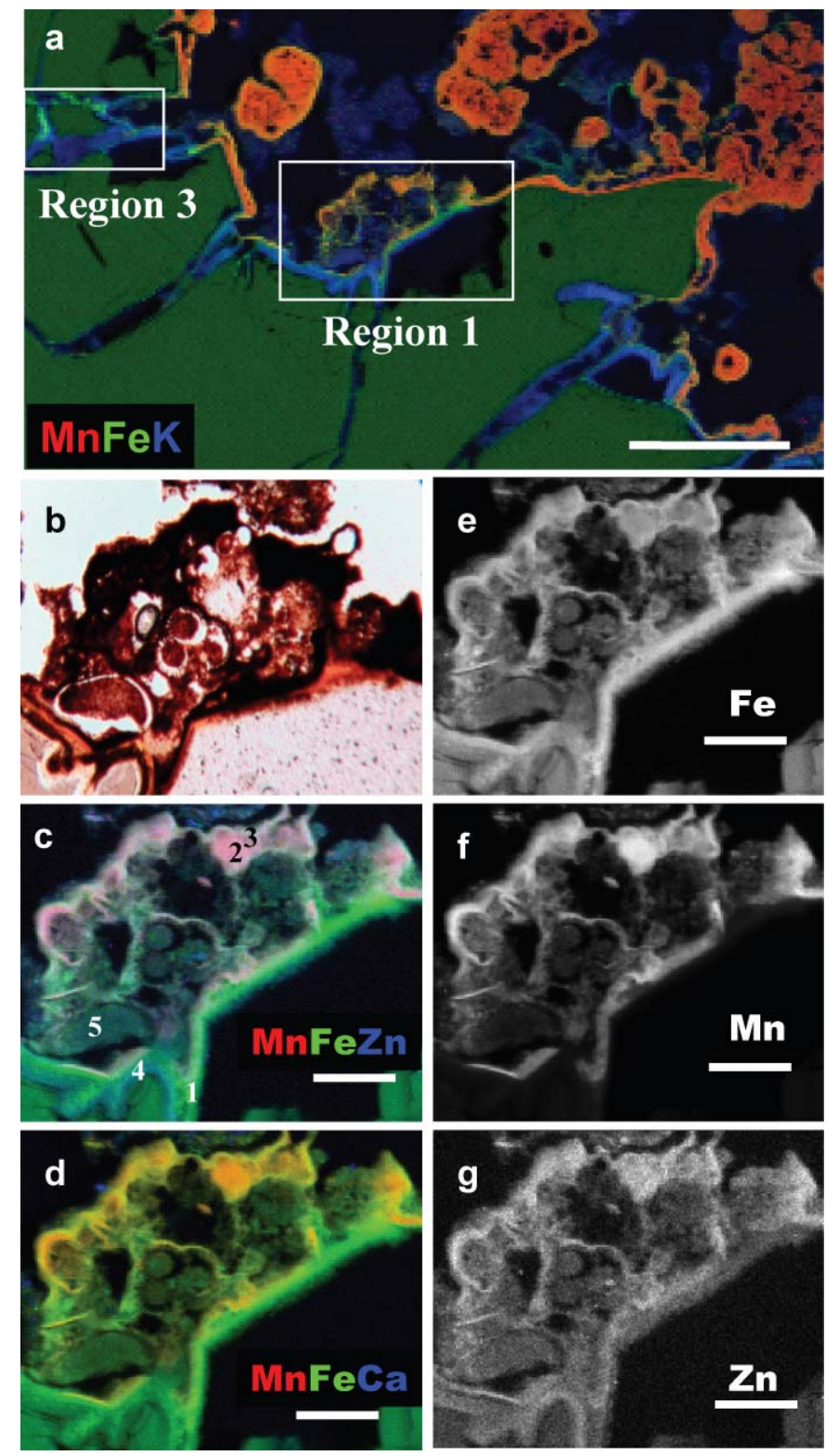

Figure 5.4. Characterization of EPR3970. (a) $\mu \mathrm{SXRF}$ tricolor map with Mn coded as red, $\mathrm{Fe}$ as green, and $\mathrm{K}$ as blue. The dark green area represents fresh basaltic glass, the blue area indicates the glass alteration layer, and the orange area represents a mixture of Fe and Mn-bearing minerals (most likely oxides). The white boxes outline the regions (1 and 3) used for more detailed analyses. Scale bar is $1 \mathrm{~mm}$. (b) Light microscope image of Region 1 in thin-section ( $30 \mu \mathrm{m}$ thick). (c-d) $\mu \mathrm{SXRF}$ tricolor maps of Region1 with Mn coded as reg, Fe as green and Zn (Fig. 5.4c) or Ca (Fig. 5.4d) as blue. The numbers (1-5) represent the locations of spot analyses used for Fe EXAFS measurements. (e-g) Grayscale maps for elements $\mathrm{Fe}, \mathrm{Mn}$, and $\mathrm{Zn}$ where bright colors indicate high concentrations. Scale bar is $200 \mu \mathrm{m}$ in images b-g. 

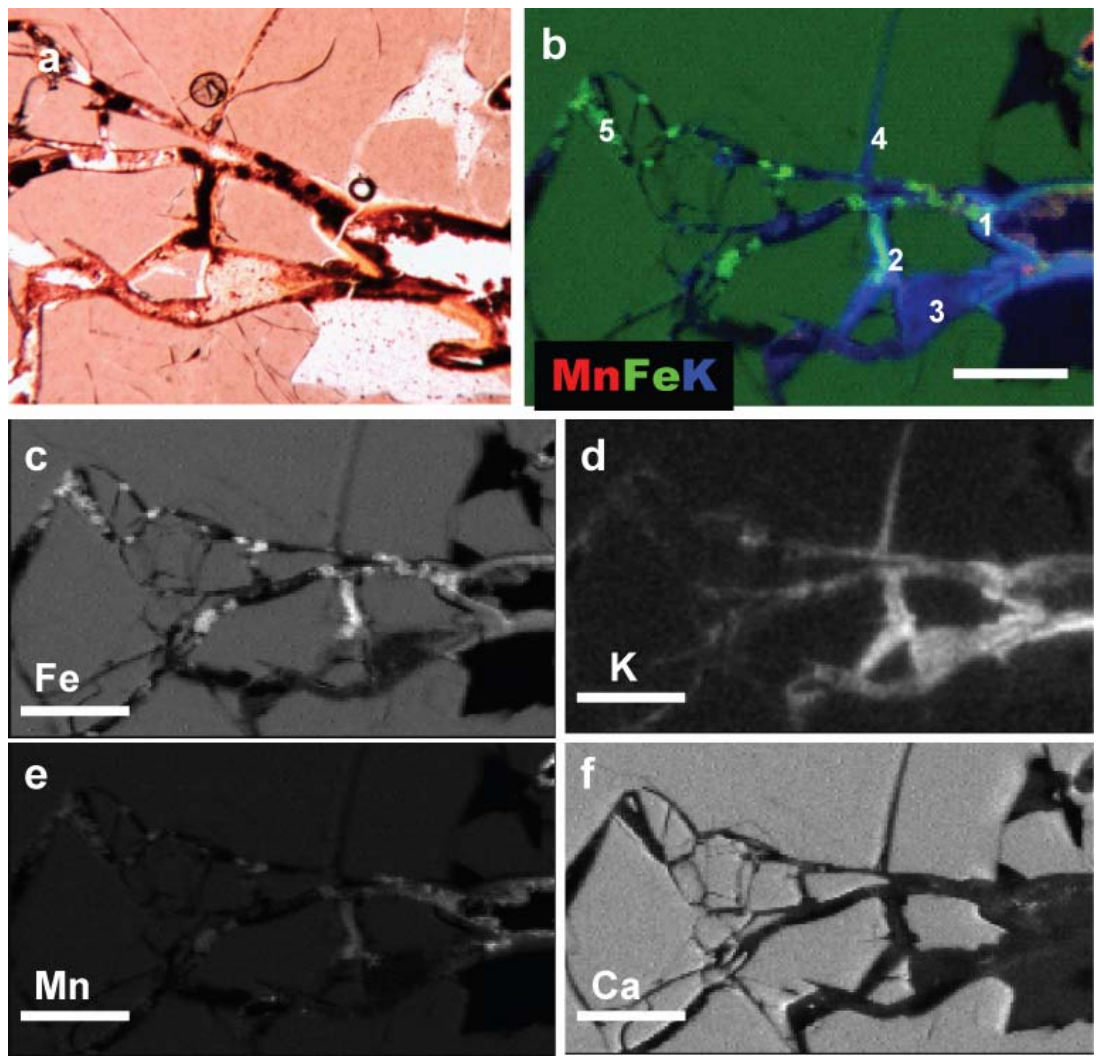

Figure 5.5. Elemental maps of EPR3970_Region3 (fractured glass filled with alteration products). (a) Light microscope image of the veins in thin-section. (b) $\mu \mathrm{SXRF}$ tricolor map with $\mathrm{Mn}$ coded as red, Fe as green, and $\mathrm{K}$ as blue. The numbers (1 -5) represent the locations of spot analyses used for Fe EXAFS measurements. (c-j) Grayscale maps for elements $\mathrm{Fe}, \mathrm{K}, \mathrm{Mn}$, and $\mathrm{Ca}$ where bright colors indicate high concentrations. Scale bar is $400 \mu \mathrm{m}$ in all images. 


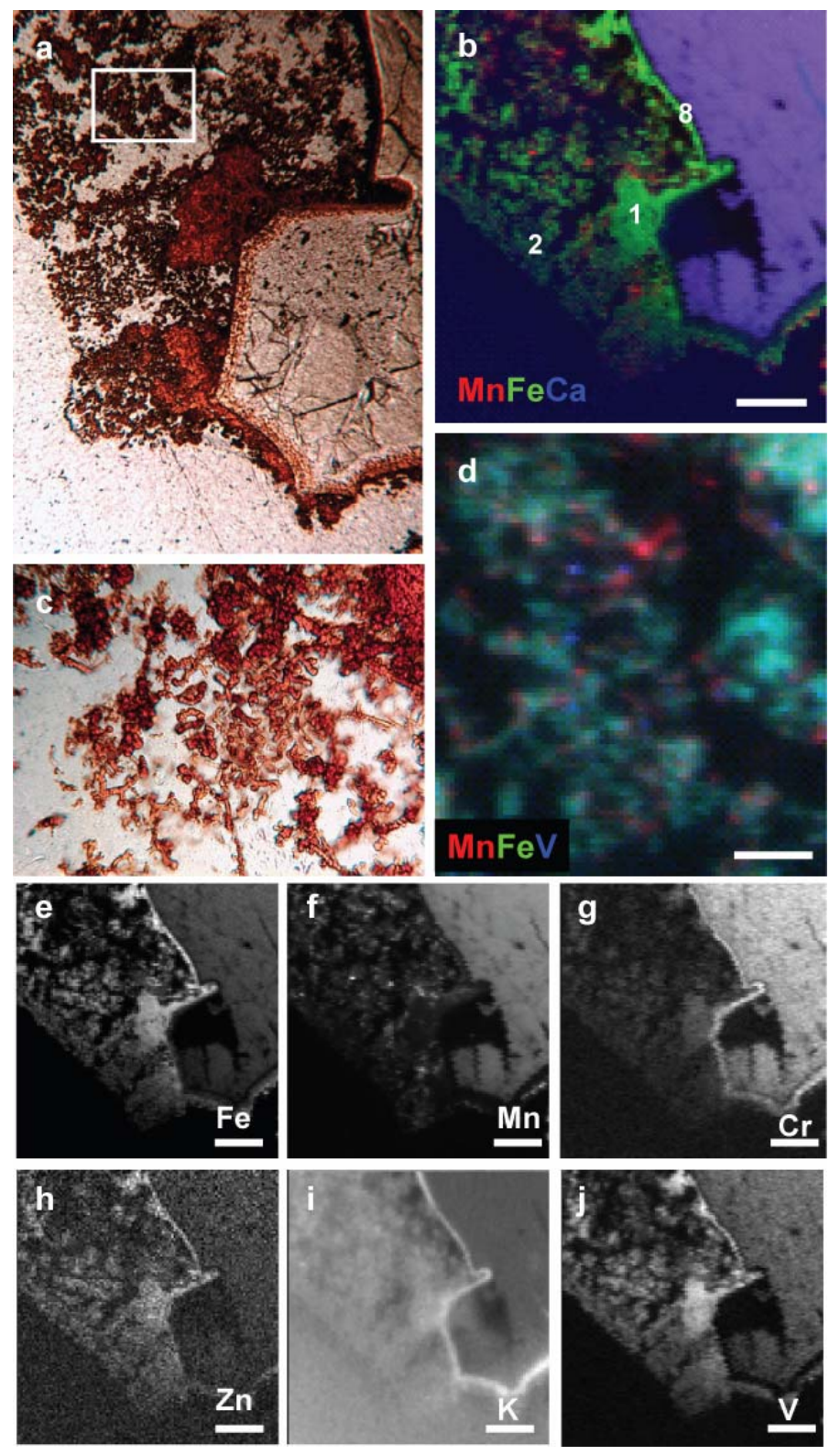

Figure 5.6. Characterization of Region 6 from sample EPR3970 (area not visible in overview map in Figure 5.3a). (a) Light microscope image of the thin section showing glass with thin alteration layer encrusted in reddish-orange minerals. (b) $\mu \mathrm{SXRF}$ tricolor map or Region 6 with $\mathrm{Mn}$ coded as red, Fe as green, and $\mathrm{Ca}$ as blue. The numbers (1-2, 8) represent the locations of spot analyses used for EXAFS measurements. (c) Higher magnification light microscope image of the area highlighted (white box) in Fig. 5.6a showing the twisted stalk morphologies of the particles. (d) High resolution $\mu \mathrm{SXRF}$ tricolor map or the oxide particles with Mn coded as red, Fe as green, and $\mathrm{V}$ as blue. Scale bar is $50 \mu \mathrm{m}$. (e-j) Grayscale maps for elements Fe, Mn, Cr, Zn, K, and V where bright colors indicate high concentrations. Scale bar is $200 \mu \mathrm{m}$ in images b, e-j. 

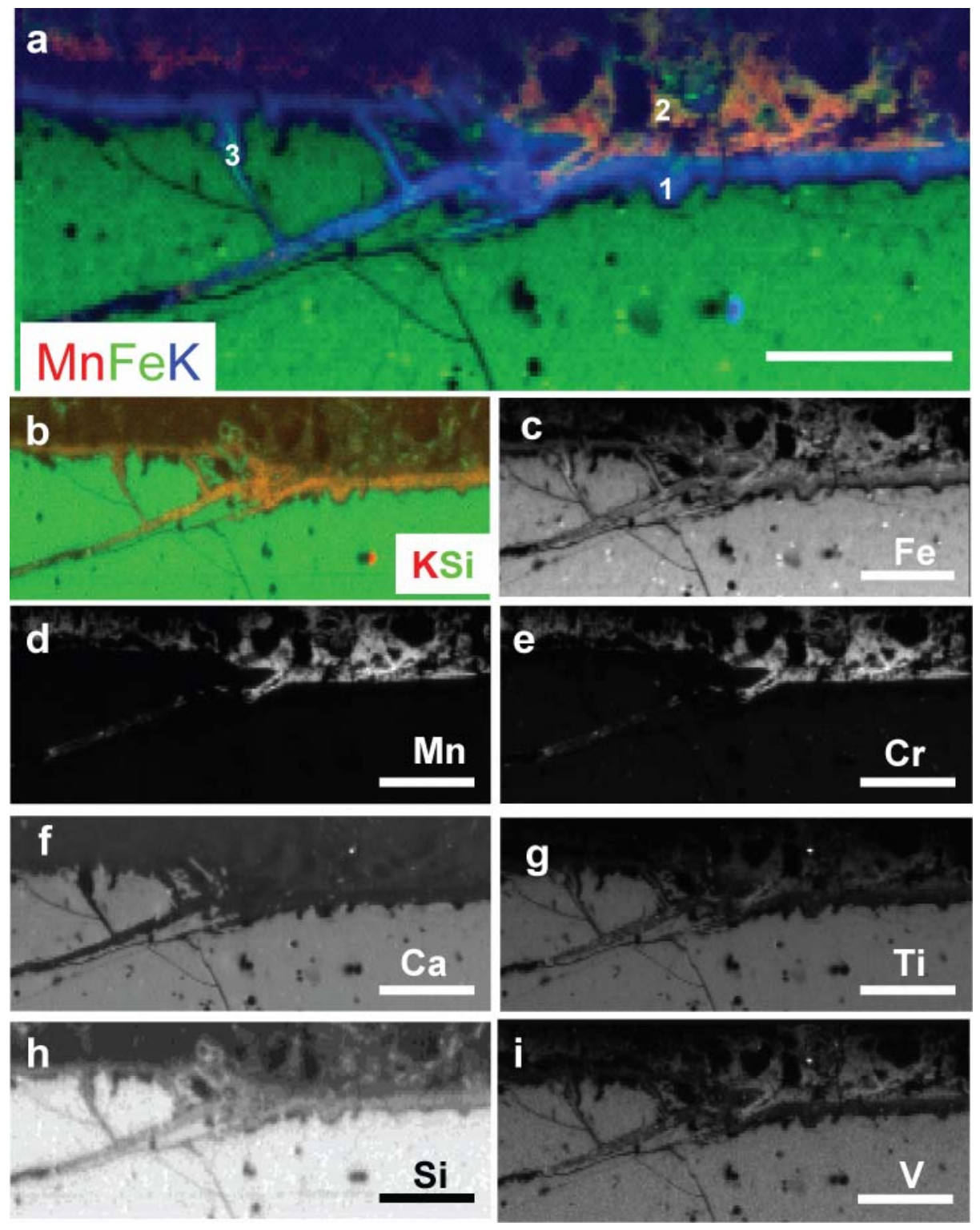

Figure 5.7. Characterization of EPR4055. (a) $\mu \mathrm{SXRF}$ tricolor map of Region 1 with Mn coded as red, $\mathrm{Fe}$ as green, and $\mathrm{K}$ as blue. The dark green area represents fresh basaltic glass, the blue area highlights the alteration products, and the orange area represents mixed Fe and Mn-oxides coating the lava surface. The numbers (1-3) represent the locations of spot analyses used for Fe EXAFS measurements. (b) $\mu$ SXRF two-color map of Region 1 with $\mathrm{K}$ coded as red and $\mathrm{Si}$ as green. (c-j) Grayscale maps for elements $\mathrm{Fe}$, $\mathrm{Mn}, \mathrm{Cr}, \mathrm{Ca}, \mathrm{Ti}, \mathrm{Xi}$, and $\mathrm{V}$ where bright colors indicate high concentrations. Scale bar is $500 \mu \mathrm{m}$ in all images. 


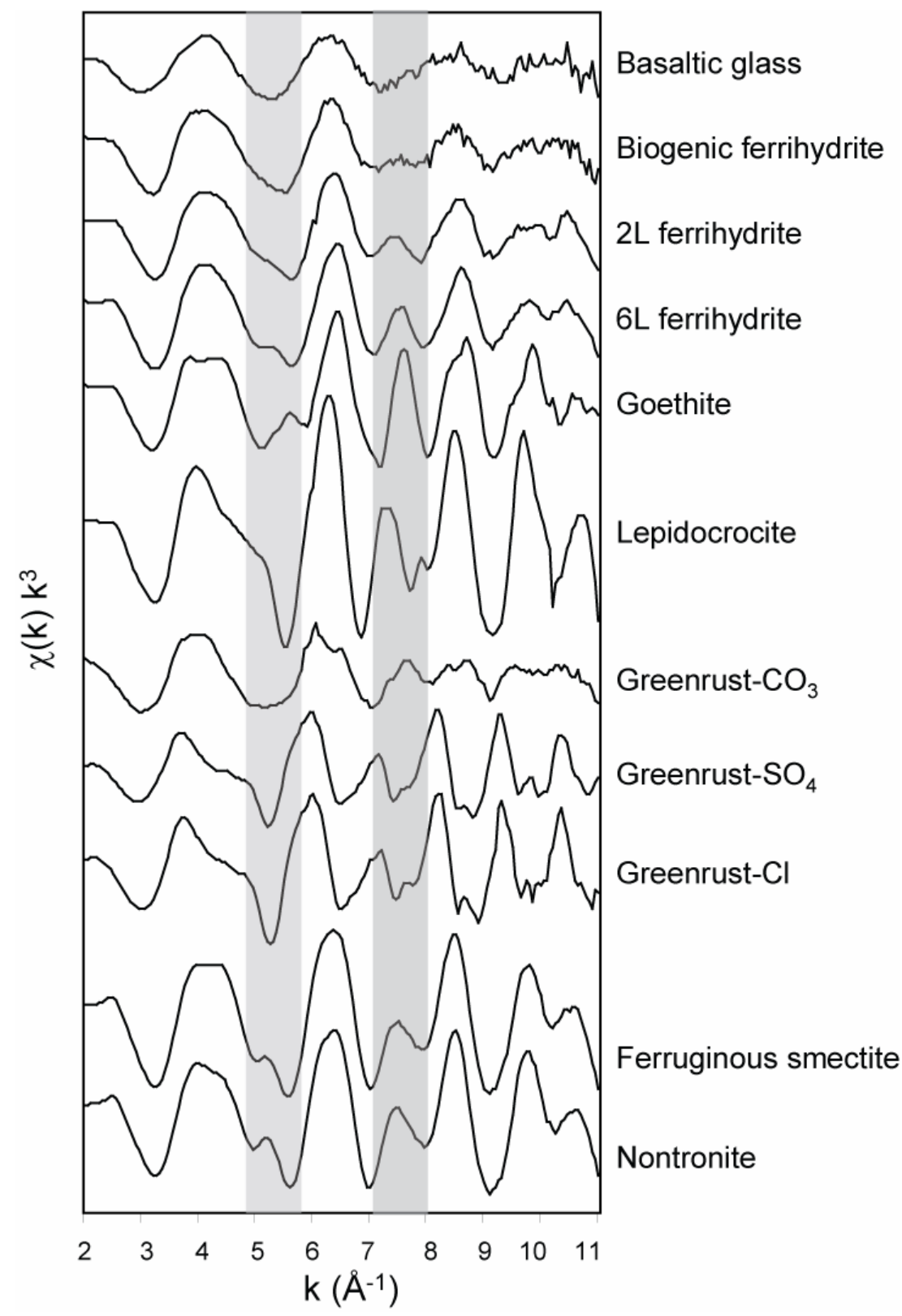

Figure 5.8. Summary of $\mathrm{k}^{3}$-weighted Fe EXAFS spectra of reference materials used for principal componenent analysis (PCA) and linear least-squares fitting. The gray boxes (indicator regions) highlight features relevant for distinguishing the $\mathrm{Fe}$ oxides and oxyhdroxides. The phase shifts and amplitude differences represent structural changes of these minerals. 


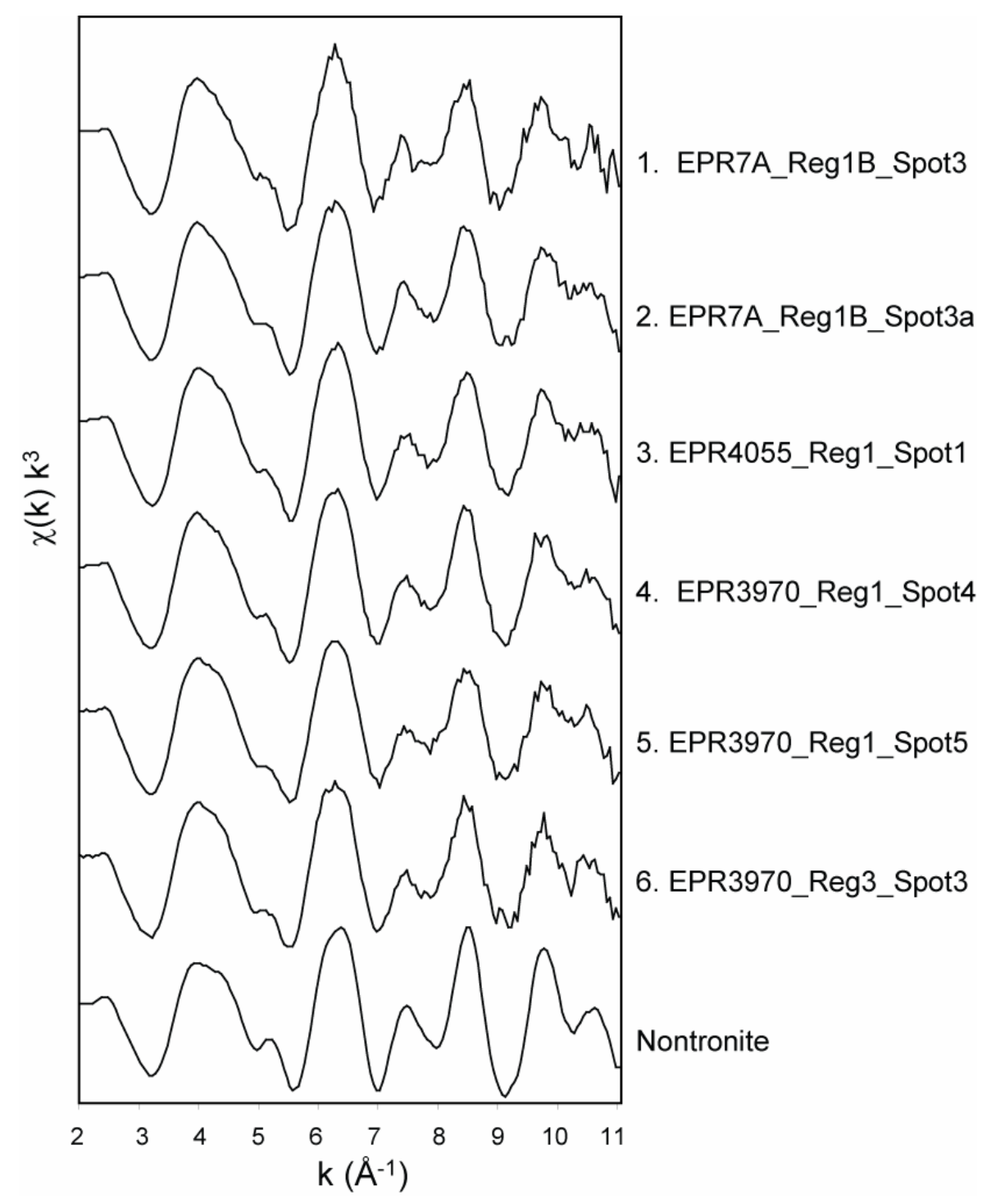

Figure 5.9. Summary of $\mathrm{k}^{3}$-weighted Fe EXAFS spectra of clay-like spectra collected from the three different altered basaltic glass samples from the seafloor at the East Pacific Rise. A nontronite reference spectrum is included to highlight similarities with the sample spectra (numbers 1-6; listed by sample name_region_spot). 


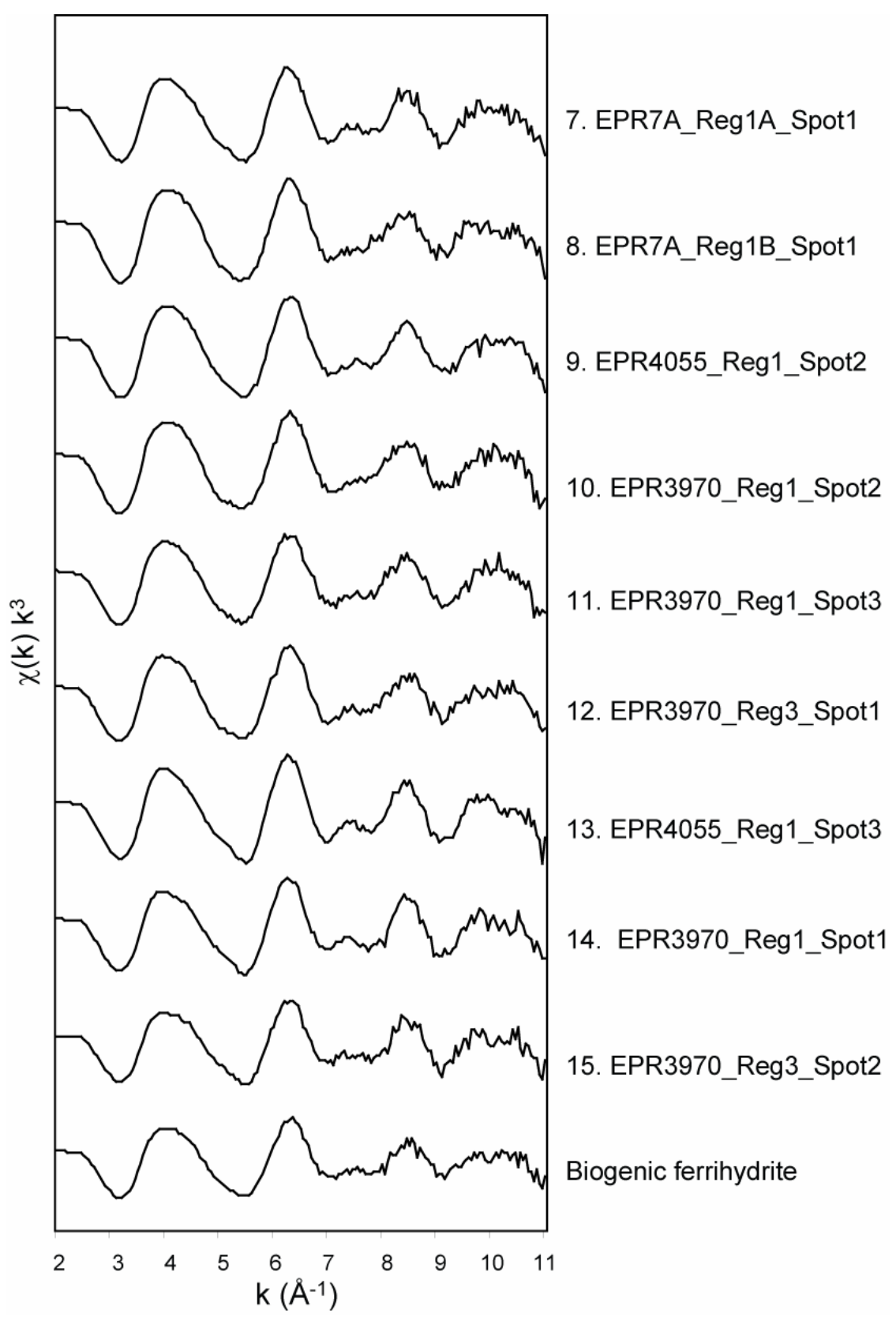

Figure 5.10. Summary of $\mathrm{k}^{3}$-weighted $\mathrm{Fe}$ EXAFS spectra resembling poorlycrystalline,Fe-oxide like spectra collected from the three different altered basaltic glass samples from the East Pacific Rise. A biogenic ferrihydrite reference spectrum is included to highlight similarities with the sample spectra (numbers 7-15). 


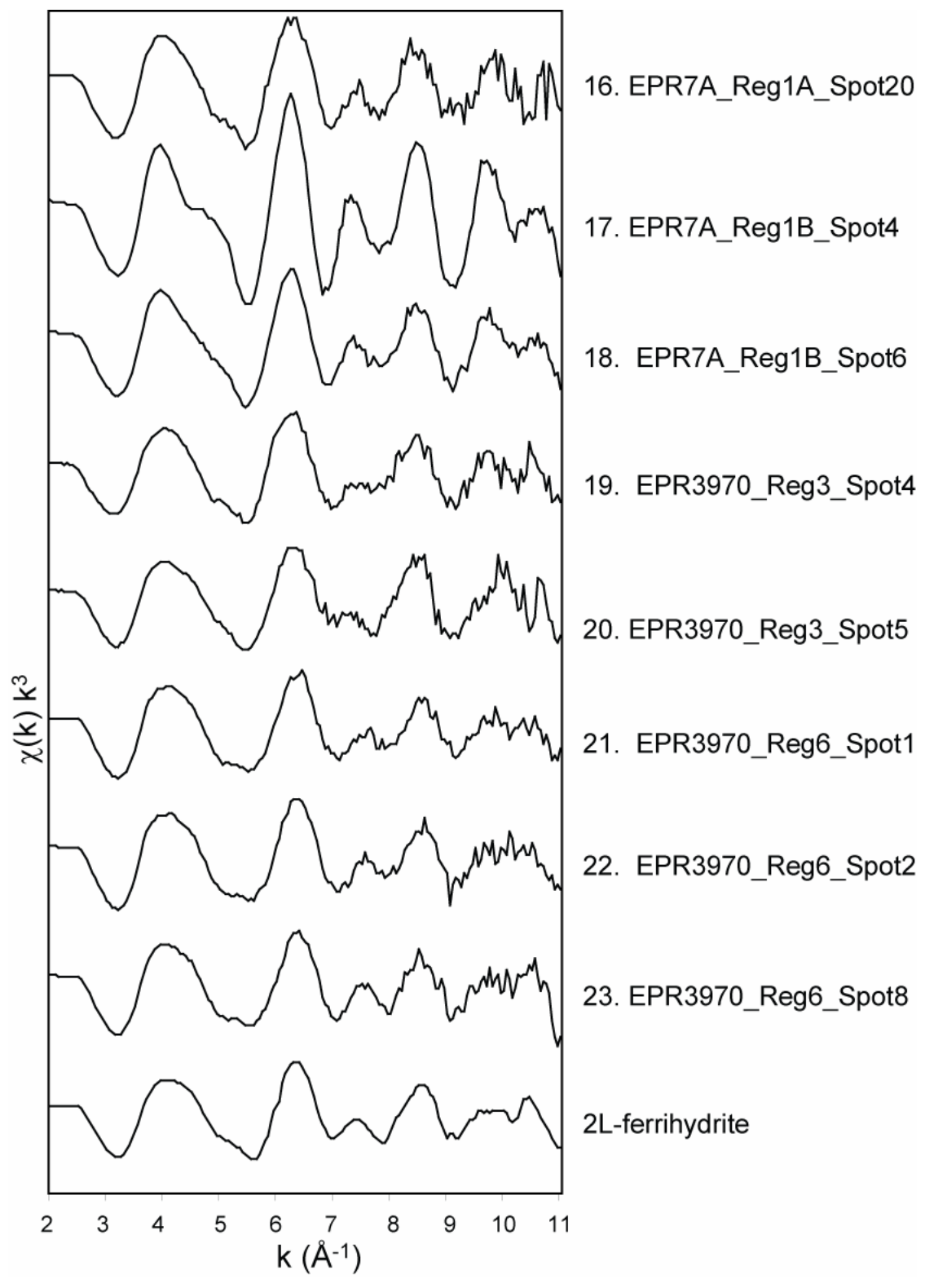

Figure 5.11. Summary of $\mathrm{k}^{3}$-weighted Fe EXAFS spectra resembling Fe-oxide-like spectra with a higher degree of polymerization or intermediate oxide/clay spectra that were collected from the three different altered basaltic glass samples from the East Pacific Rise. A 2L-ferrihydrite reference spectra is included to highlight similarities and differences with the sample spectra (numbers 16-23). 
A

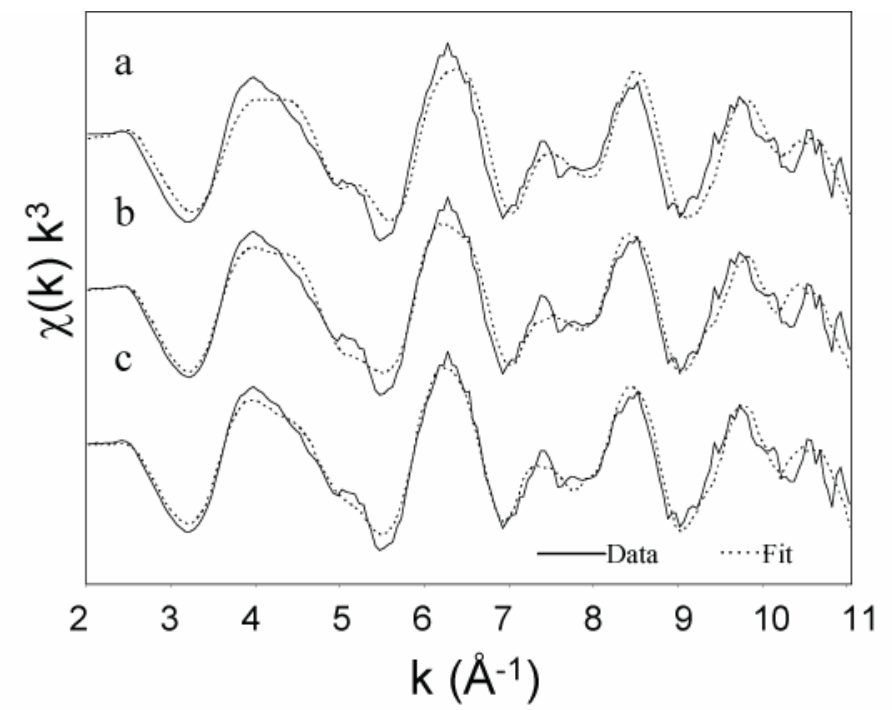

\begin{tabular}{|ccccccc|}
\hline & \multicolumn{3}{c}{ Spectral components } & & \\
\cline { 2 - 4 } $\begin{array}{c}\# \\
\text { components }\end{array}$ & $\begin{array}{c}\text { ferruginous } \\
\text { smectite }\end{array}$ & $\begin{array}{c}\text { greenrust- } \\
\text { SO4 }\end{array}$ & lepidocrocite & SUM & $\chi^{2}$ \\
\hline 1 & 0.82 & ---- & ---- & 0.82 & 1.08 \\
2 & 0.81 & 0.31 & ---- & 1.12 & 0.72 \\
3 & 0.54 & 0.31 & 0.24 & 1.10 & 0.31 \\
\hline
\end{tabular}

Figure 5.12. (A) Linear least-squares fits to $\mathrm{k}^{3}$-weighted Fe EXAFS spectrum collected from EPR7A_Reg1B_Spot3 (spectrum \#1). The data (solid line) are compared to the best spectral fits (dotted line) for a single component (a), two components (b), and three components (c). (B) Fraction of each component used for spectral fits, the sum of each component (not forced to equal one), and the reduced chi-squared parameter as an indication of the goodness of fit. 


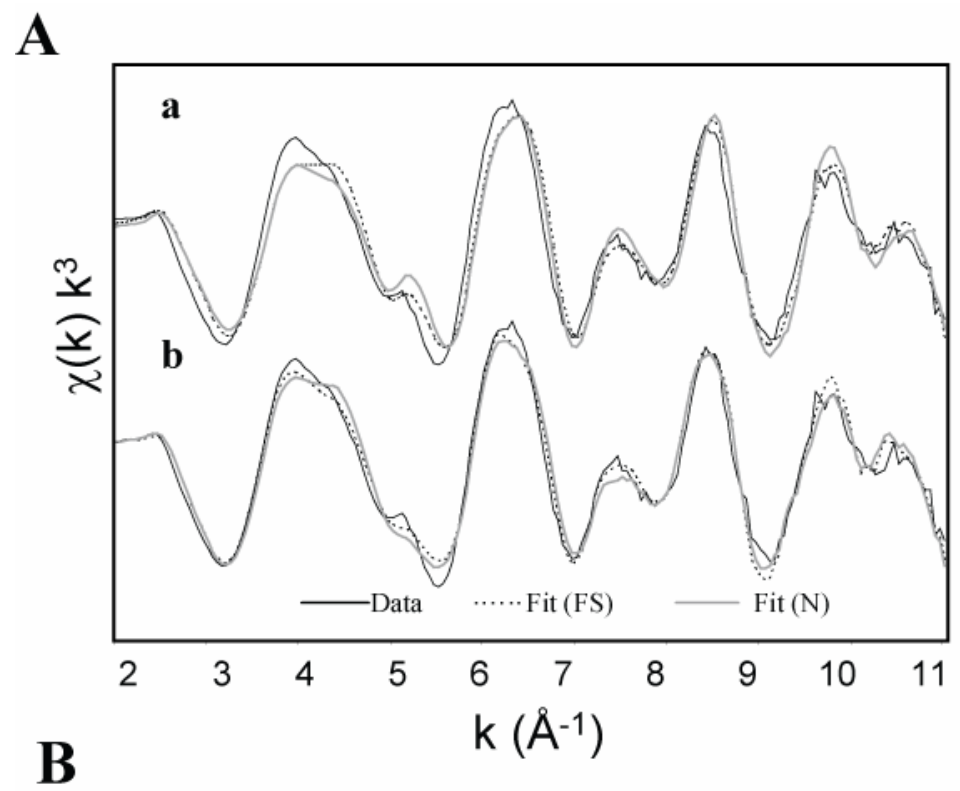

\begin{tabular}{|ccccc|}
\hline \multirow{2}{*}{$\begin{array}{c}\text { \# } \\
\text { components }\end{array}$} & $\begin{array}{c}\text { ferruginous } \\
\text { smectite }\end{array}$ & greenrust-Cl & SUM & $\chi^{2}$ \\
\hline 1 & 0.87 & ---- & 0.87 & 0.68 \\
2 & 0.84 & 0.22 & 1.07 & 0.38 \\
& nontronite & greenrust-Cl & & \\
\hline 1 & 0.92 & ----- & 0.92 & 0.86 \\
2 & 0.89 & 0.31 & 1.20 & 0.27 \\
\hline
\end{tabular}

Figure 5.13. (A) Linear least-squares fits to $\mathrm{k}^{3}$-weighted Fe EXAFS spectrum collected from EPR3970_Reg1_Spot4 (spectrum \#4), taken as a representative for spectra \#2-6. The data (solid line) are compared to the best spectral fits for a single component (a) and two components (b) using ferruginous smectite (dotted line) or nontonite (gray line) as the primary clay component. (B) Fraction of each component used for spectral fits, the sum of each component (not forced to equal one), and the reduced chi-squared parameter as an indication of the goodness of fit. 


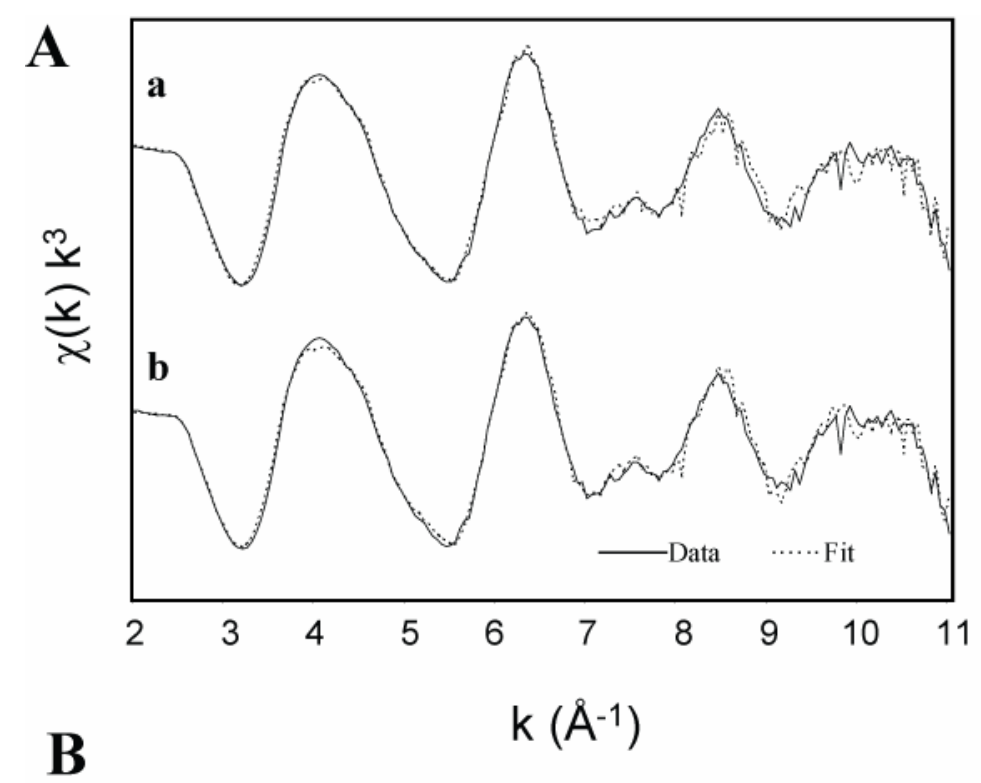

\begin{tabular}{|ccccc|}
\hline \multirow{2}{*}{ \# components } & \multicolumn{2}{c}{ Spectral components } & & \\
\cline { 2 - 3 } & $\begin{array}{c}\text { biogenic } \\
\text { ferrihydrite }\end{array}$ & $\begin{array}{c}\text { ferruginous } \\
\text { smectite }\end{array}$ & SUM & $\chi^{2}$ \\
\hline 1 & 1.30 & $-\cdots--$ & 1.30 & 0.17 \\
2 & 1.05 & 0.14 & 1.19 & 0.13 \\
\hline
\end{tabular}

Figure 5.14. (A) Linear least-squares fits to $\mathrm{k}^{3}$-weighted Fe EXAFS spectrum collected from EPR4055_Reg1_Spot2 (spectrum \#9), taken as a representative for spectra \#7-12. The data (solid line) are compared to the best spectral fits (dotted line) for a single component (a) and two components (b). (B) Fraction of each component used for spectral fits, the sum of each component (not forced to equal one), and the reduced chisquared parameter as an indication of the goodness of fit. 


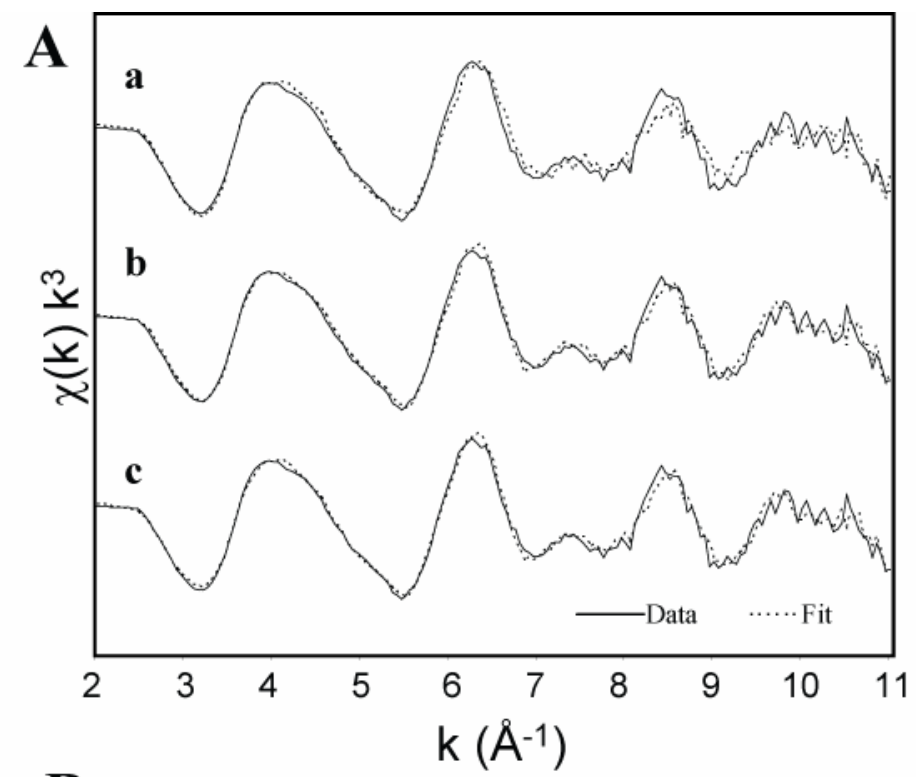

B

\begin{tabular}{|cccccc|}
\hline & \multicolumn{3}{c}{ Spectral components } & & \\
\cline { 2 - 5 } $\begin{array}{c}* \\
\text { components }\end{array}$ & $\begin{array}{c}\text { biogenic } \\
\text { ferrihydrite }\end{array}$ & lepidocrocite & $\begin{array}{c}\text { basaltic } \\
\text { glass }\end{array}$ & SUM & $\chi^{2}$ \\
\hline 1 & 1.18 & ---- & ---- & 1.18 & 0.30 \\
2 & 0.90 & 0.14 & ---- & 1.04 & 0.15 \\
3 & 0.73 & 0.15 & 0.24 & 1.12 & 0.14 \\
\hline
\end{tabular}

Figure 5.15. (A) Linear least-squares fits to $\mathrm{k}^{3}$-weighted Fe EXAFS spectrum collected from EPR3970_Reg1_Spot1 (spectrum \#14), taken as a representative for spectra \#13-15. The data (solid line) are compared to the best spectral fits (dotted line) for a single component (a), two components (b), and 3 components (c). (B) Fraction of each component used for spectral fits, the sum of each component (not forced to equal one), and the reduced chi-squared parameter as an indication of the goodness of fit. 


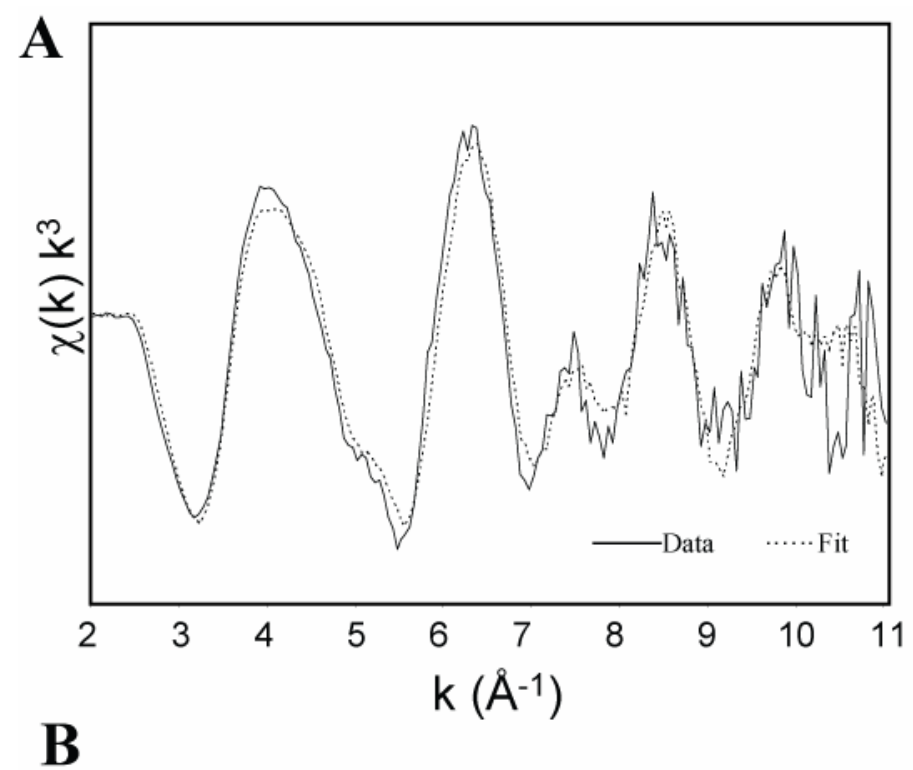

\begin{tabular}{|cccccc|}
\hline \multirow{4}{*}{ \# components } & \begin{tabular}{c}
\multicolumn{2}{c}{ Spectral components } \\
biogenic \\
\cline { 2 - 5 } \\
ferrihydrite
\end{tabular} & nontronite & SUM & $\chi^{2}$ \\
\hline 2 & 0.85 & 0.31 & 1.16 & 0.84 \\
\hline
\end{tabular}

Figure 5.16. (A) Linear least-squares fits to $\mathrm{k}^{3}$-weighted Fe EXAFS spectrum collected from EPR7A_Reg1A_Spot20 (spectrum \#16). The data (solid line) are compared to the best spectral fit (dotted line) for a two component fit only (which was the substantially better fit). (B) Fraction of each component used for spectral fits, the sum of each component (not forced to equal one), and the reduced chi-squared parameter as an indication of the goodness of fit. 


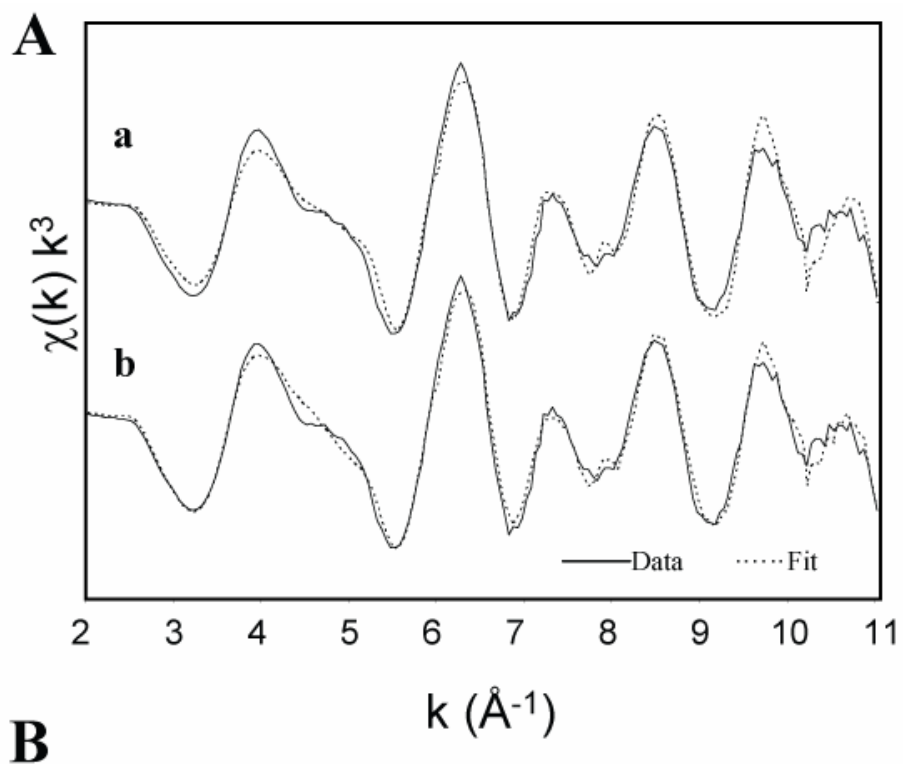

\begin{tabular}{|ccccc|}
\hline & \multicolumn{2}{c}{ Spectral components } & & \\
\cline { 2 - 4 } $\begin{array}{c}\text { components } \\
\text { connnn}\end{array}$ & lepidocrocite & $\begin{array}{c}\text { biogenic } \\
\text { ferrihydrite }\end{array}$ & SUM & $\chi^{2}$ \\
\hline 1 & 0.83 & ---- & 0.83 & 0.62 \\
2 & 0.67 & 0.49 & 1.17 & 0.36 \\
\hline
\end{tabular}

Figure 5.17. (A) Linear least-squares fits to $\mathrm{k}^{3}$-weighted Fe EXAFS spectrum collected from EPR7A_Reg1B_Spot4 (spectrum \#17). The data (solid line) are compared to the best spectral fits (dotted line) for a single component (a) and two components (b). (B) Fraction of each component used for spectral fits, the sum of each component (not forced to equal one), and the reduced chi-squared parameter as an indication of the goodness of fit. 


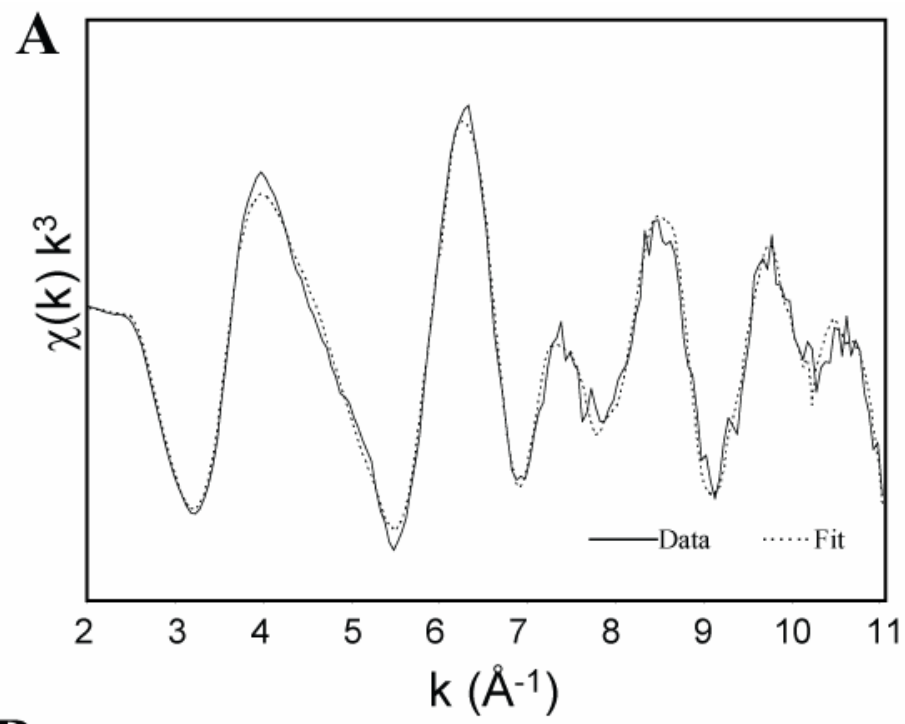

B

\begin{tabular}{|ccccccc|}
\hline & \multicolumn{4}{c}{ Spectral components } & & \\
\cline { 2 - 5 } $\begin{array}{c}\# \\
\text { components }\end{array}$ & 6L-ferrihydrite & greenrust-Cl & lepidocrocite & SUM & $\chi^{2}$ \\
\hline 3 & 0.45 & 0.23 & 0.33 & 1.01 & 0.13 \\
\hline
\end{tabular}

Figure 5.18. (A) Linear least-squares fits to $\mathrm{k}^{3}$-weighted Fe EXAFS spectrum collected from EPR7A_Reg1B_Spot6 (spectrum \#18). The data (solid line) are compared to the best spectral fit (dotted line) for a three component fit only (which was the substantially better fit). (B) Fraction of each component used for spectral fits, the sum of each component (not forced to equal one), and the reduced chi-squared parameter as an indication of the goodness of fit. 


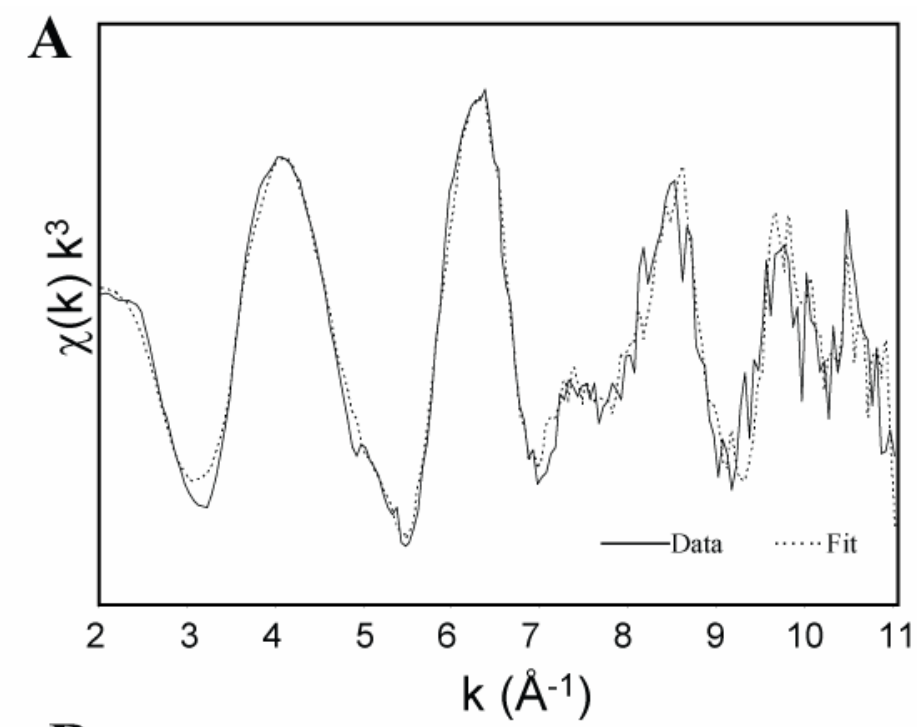

B

\begin{tabular}{|ccccc|}
\hline & \multicolumn{2}{c}{ Spectral components } & & \\
\cline { 2 - 4 } $\begin{array}{c}* \\
\text { components }\end{array}$ & $\begin{array}{c}\text { basaltic } \\
\text { glass }\end{array}$ & lepidocrocite & SUM & $\chi^{2}$ \\
\hline 2 & 1.01 & 0.24 & 1.24 & 0.37 \\
\hline
\end{tabular}

Figure 5.19. (A) Linear least-squares fits to $\mathrm{k}^{3}$-weighted Fe EXAFS spectrum collected from EPR3970_Reg3_Spot4 (spectrum \#19). The data (solid line) are compared to the best spectral fits (dotted line) for a two component fit only (which was the substantially better fit). (B) Fraction of each component used for spectral fits, the sum of each component (not forced to equal one), and the reduced chi-squared parameter as an indication of the goodness of fit. 


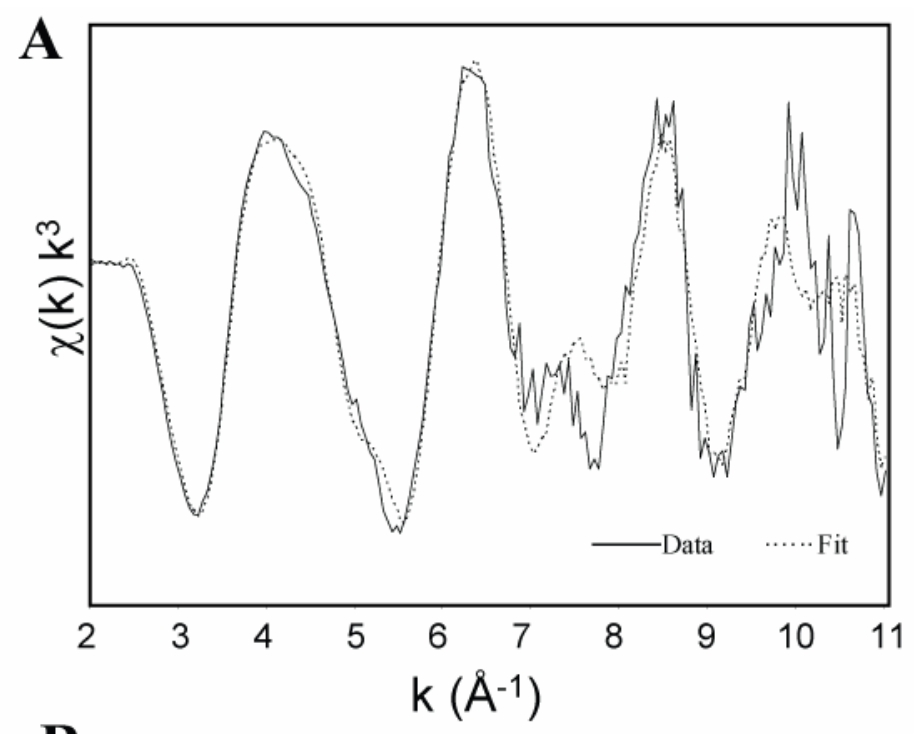

B

\begin{tabular}{|ccccc|}
\hline \multirow{2}{*}{$\begin{array}{c}\text { \# } \\
\text { components }\end{array}$} & $\begin{array}{c}\text { Spectral components } \\
\text { biogenic } \\
\text { ferrihydrite }\end{array}$ & $\begin{array}{c}\text { ferruginous } \\
\text { smectite }\end{array}$ & SUM & $\chi^{2}$ \\
\hline 2 & 0.59 & 0.33 & 0.92 & 0.60 \\
\hline
\end{tabular}

Figure 5.20. (A) Linear least-squares fits to $\mathrm{k}^{3}$-weighted Fe EXAFS spectrum collected from EPR3970_Reg3_Spot5 (spectrum \#20). The data (solid line) are compared to the best spectral fit (dotted line) for a two component fit only (which was the substantially better fit). (B) Fraction of each component used for spectral fits, the sum of each component (not forced to equal one), and the reduced chi-squared parameter as an indication of the goodness of fit. 


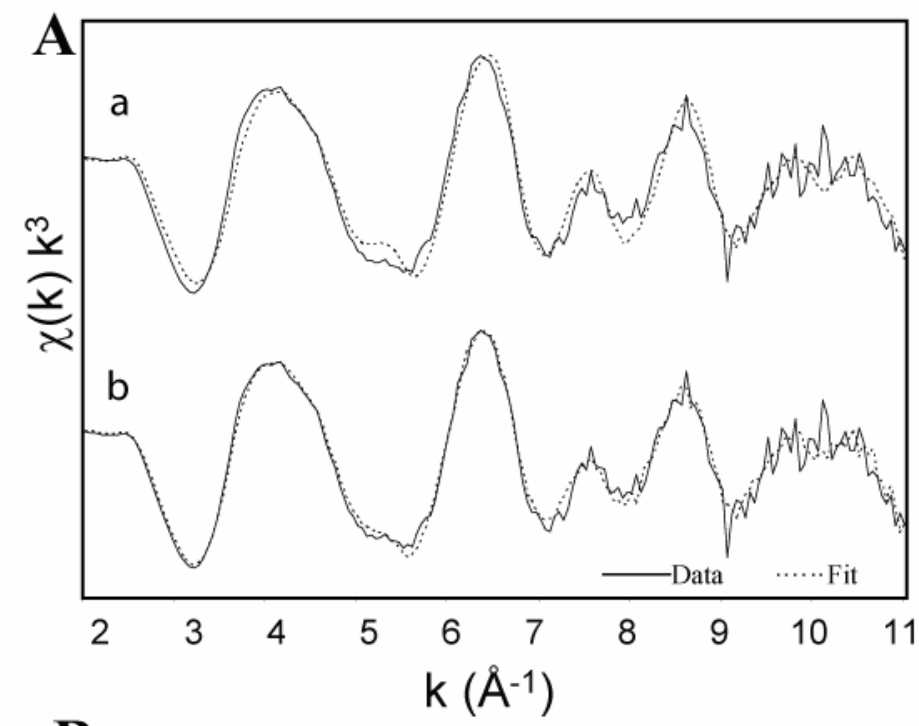

B

\begin{tabular}{|ccccc|}
\hline & \multicolumn{2}{c}{ Spectral components } & & \\
\cline { 2 - 3 } \# components & $\begin{array}{c}6 \mathrm{~L}- \\
\text { ferrihydrite }\end{array}$ & $\begin{array}{c}\text { biogenic } \\
\text { ferrihydrite }\end{array}$ & SUM & $\chi^{2}$ \\
\hline 1 & 0.92 & ---- & 0.92 & 0.31 \\
2 & 0.51 & 0.58 & 1.09 & 0.19 \\
\hline
\end{tabular}

Figure 5.21. (A) Linear least-squares fits to $\mathrm{k}^{3}$-weighted Fe EXAFS spectrum collected from EPR3970_Reg6_Spot2 (spectrum \#22), taken as a representative for spectra \#21-23. The data (solid line) are compared to the best spectral fits (dotted line) for a single component (a) and two components (b). (B) Fraction of each component used for spectral fits, the sum of each component (not forced to equal one), and the reduced chisquared parameter as an indication of the goodness of fit. 


\subsection{Appendix}

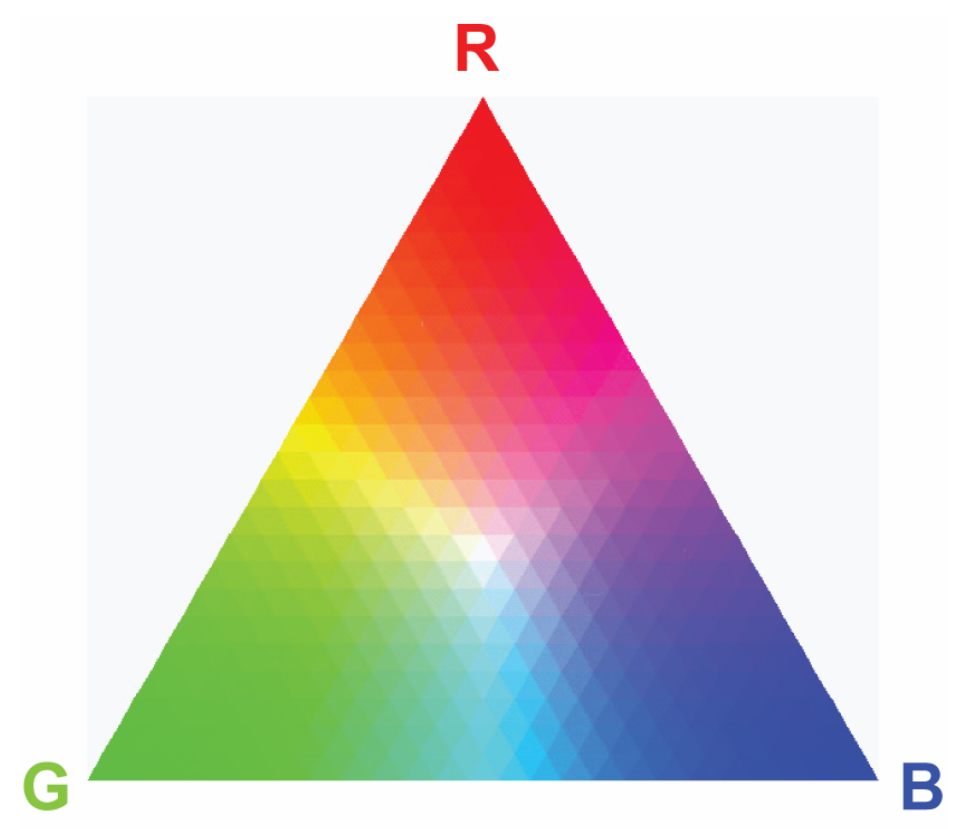

Figure A5.1. Red-Green-Blue color triangle depicting the color spectra produced from mixing (co-location) of different proportions of the end-member color choices (representing specific elements). For example, the co-location of red and green elements produces yellow, co-location of red and blue produces magenta, and green and blue produces cyan. Co-location of all three end-member in approximately equal proportions elements produces white. 


\subsection{References}

Alt, J.C., 1995. Subseafloor processes in mid-ocean ridge hydrothermal systems. In: S.E. Humphris, R.A. Zierneberg, L.S. Mullineaux and R.E. Thomson (Editors), Seafloor hydrothermal systems: physical, chemical, biological, and geological interactions. AGU Monograph. American Geophysical Union, Washington, DC, pp. 85-114.

Alt, J.C. and Mata, P., 2000. On the role of microbes in the alteration of submarine basaltic glass: a TEM study. Earth and Planetary Science Letters, 181(301-313).

Alt, J.C. and Teagle, D.A., 1999. The uptake of carbon during alteration of ocean crust. Geochim. Cosmochim. Acta, 63(20): 1527-1535.

Arrhenius, G.O., 2003. Crystals and Life. Helvetica Chimica Acta, 86: 1569-1586.

Bach, W. and Edwards, K.J., 2003. Iron and sulfide oxidation within the basaltic ocean crust: Implications for chemolithoautotrophic microbial biomass production. Geochim. Cosmochim. Acta, 67(20): 3871-3887.

Bach, W., Peucker-Ehrenbrink, B., Hart, S.R. and Blusztajn, J.S., 2003. Geochemistry of hydrothermally altered oceanic crust: DSDP/ODP Hole 504B - Implications for seawater-crust exchange budgets and $\mathrm{Sr}$ - and $\mathrm{Pb}$-isotopic evolution of the mantle. GEOCHEMISTRY GEOPHYSICS GEOSYSTEMS, 4(3): 1-29.

Banerjee, N.R. and Muehlenbachs, K., 2003. Tuff life: Bioalteration in volcaniclastic rocks from the Ontong Java Plateau. Geochemistry Geophysics Geosystems, 4: Art. No. 1037(art. no. 1037).

Banfield, J.F., Welch, S.A., Zhang, H.Z., Thornsen Ebert, T. and Penn, R.L., 2000. Aggregation-based crystal growth and microstructure development in natural iron oxyhydroxide biomineralization products. Science, 289: 751-754.

Bernal, J.D.H., Dasqupta, D.R. and Mackay, A.L., 1959. The oxides and hydroxides of iron and their structural inter-relationships. Clay Mineral. Bull., 4: 15-30.

Carlson, L. and Schwertmann, U., 1980. Natural occurrence of feroxyhite (delta'FeOOH). Clays and Clay Minerals, 28(4): 272-280.

Crovisier, J.-L., Advocat, T. and Dussossoy, J.-L., 2003. Nature and role of natural alteration gels formed on the surface of ancient volcanic glasses (Natural analogs of waste containment glasses). Journal of Nuclear Materials, 321: 91-109.

Crovisier, J.-L., Honnorez, J. and Eberhart, J.P., 1987. Dissolution of basaltic glass in seawater: mechanism and rate. Geochim. Cosmochim. Acta, 51(11): 2977-2990.

Daughney, C.J., Rioux, J.-P., Fortin, D. and Pichler, T., 2004. Laboratory Investigation of the Role of Bacteria in the Weathering of Basalt Near Deep Sea Hydrothermal Vents. Geomicrobiology Journal, 21: 21-31.

Daux, V., Guy, C., Advocat, T., Crovisier, J.-L. and Stille, P., 1997. Kinetic aspects of basaltic glass dissolution at $90^{\circ} \mathrm{C}$ : role of aqueous silicon and aluminium.

Chemical Geology, 142: 109-126.

Edmonds, H.N. and German, C.R., 2004. Particle geochemistry in the Rainbow hydrothermal plume, Mid-Atlantic Ridge. Geochim. Cosmochim. Acta, 68(4): 759-772. 
Edwards, K.J., Bach, W. and McCollom, T.M., 2005. Geomicrobiology in oceanography: microbe-mineral interactions at and below the seafloor. TRENDS in Microbiology, 13(9).

Edwards, K.J., Bach, W., McCollom, T.M. and Rogers, D.R., 2004. Neutrophilic ironoxidizing bacteria in the ocean: Their habitats, diversity, and roles in mineral deposition, rock alteration, and biomass production in the deep-sea. Geomicrobiology Journal, 21: 393-404.

Edwards, K.J., McCollom, T.M., Konishi, H. and Buseck, P.R., 2003. Seafloor bioalteration of sulfide minerals: results from in situ incubation studies. Geochim. Cosmochim. Acta, 67(15): 2843-2856.

Elderfield, H. and Schultz, A., 1996. Mid-ocean ridge hydrothermal fluxes and the chemical composition of the ocean. Annu. Rev. Earth Planet. Sci., 24: 191-224.

Emerson, D. and Moyer, C.L., 2002. Neutrophilic Fe-oxidizing bacteria are abundant at the Loihi Seamount hydrothermal vents and play a major role in Fe oxide deposition. Applied and Environmental Microbiology, 68(6): 3085-3093.

Fisher, A.T. and Becker, K., 2000. Channelized fluid flow in oceanic crust reconciles heat-flow and permeability data. Nature, 403: 71-74.

Giorgetti, G., Marescotti, P., Cabella, R. and Lucchetti, G., 2001. Clay mineral mixtures as alteration products in pillow basalts from the eastern flank of Juan de Fuca Ridge: a TEM-AEM study. Clay Minerals, 36(75-91).

Gislason, S.R. and Arnorsson, S., 1993. Dissolution of primary basaltic minerals in natural waters: saturation state and kinetics. Chem. Geol., 105: 117-135.

Gislason, S.R. and Oelkers, E.H., 2000. Chemical Weathering rate of basaltic glass as a function of temperature, $\mathrm{pH}$, organic acids and solution composition. Journal of Conference Abstracts, 5(2): 443.

Guy, C. and Schott, J., 1989. Multisite surface reaction versus transport control during the hydrolysis of a complex oxide. Chemical Geology, 78: 181-204.

Hansel, C.M. et al., 2003. Secondary mineralization pathways induced by dissimilatory iron reduction of ferrihydrite under advective flow. Geochimica et Cosmochimica Acta, 67(16): 2977-2992.

Humphris, S. and Thompson, G., 1978. Hydrothermal alteration of oceanic basalts by seawater. Geochimica et Cosmochimica Acta, 42(1): 107-125.

Keeling, J.L., Raven, M.D. and Gates, W.P., 2000. Geology and characterization of two hydrothermal nontronites from weathered metamorphic rocks at the Uley Graphite Mine, South Australia. Clays and Clay Minerals, 48(5): 537-548.

Malinowski, E.R., 1978. Theory of error for target factor analysis with application to mass spectrometry and nuclear magnetic resonance spectrometry. Anal. Chim. Acta, 103: 339-354.

Manceau, A. et al., 1996. Direct determination of lead speciation in contaminated soils by EXAFS spectroscopy. Env. Sci. Tech., 30: 1540-1552.

Manceau, A. and Drits, V.A., 1993. Local structure of ferrihydrite and feroxyhite by EXAFS spectroscopy. Clay Minerals, 28: 165-184. 
Manceau, A. et al., 2000. Oxidation-reduction mechanism of iron in dioctahedral smectites: 1. Crystal chemistry of oxidized reference nontronites. American Mineralogist, 85: 133-152.

Manceau, A., Marcus, M.A. and Tamura, N., 2002. Quantitative speciation of heavy metals in soils and sediments by synchrotron X-ray techniques. In: P.A. Fenter, M.L. Rivers, N.C. Sturchio and S.R. Sutton (Editors), Applications in lowtemperature geochemistry and environmental science. Reviews in mineralogy and geochemistry. Mineralogical Society of America, Washington D.C., pp. 341-428.

Marcus, M.A. et al., 2004a. Beamline 10.3.2 at ALS: a hard X-ray microprobe for environmental and materials sciences. J. Synch. Rad., 11: 239-247.

Marcus, M.A., Manceau, A. and Kersten, M., 2004b. Mn, Fe, Zn, and As speciation in a fast-growing ferromanganese marine nodule. Geochim. Cosmochim. Acta, 68(14): 3125-3136.

Newville, M., 2001. IFEFFIT: interactive XAFS analysis and FEFF fitting. J. Synch. Rad., 8: 324-332.

Oelkers, E.H., 2001. General kinetic description of multioxide silicate mineral and glass dissolution. Geochimica et Cosmochimica Acta, 65(21): 3703-3719.

Oelkers, E.H. and Gislason, S.R., 2001. The mechanism, rates and consequences of basaltic glass dissolution: I. An experimental study of the dissolution rates of basaltic glass as a function of aqueous $\mathrm{Al}, \mathrm{Si}$ and oxalic acid concentration at $25 \mathrm{C}$ and $\mathrm{pH}=3$ and 11. Geochimica et Cosmochimica Acta, 65(21): 3671-3681.

Pichler, T., Ridley, W.I. and Nelson, E., 1999. Low-temperature alteration of dredged volcanics from the Southern Chile Ridge: additional information about early stages of seafloor weathering. Marine Geology, 159: 155-177.

Porter, S., Vanko, D.A. and Ghazi, A.M., 2000. Major and trace element compositions of secondary clays in basalts altered at low temperature, eastern flank of the Juan de Fuca Ridge. In: A.T. Fisher, E.E. Davis and C. Escutia (Editors), Proceedings of the Ocean Drilling Program, Scientific Results.

Ravel, B., 2000. EXAFS analysis using FEFF and FEFFIT. A workshop with course materials available on CD-ROM at ,http://feff.Phys.washington.edu/ravel/course/>.

Schramm, B., Devey, C.W., Gillis, K.M. and Lackshewitz, K., 2005. Quantitative assessment of chemical and mineralogical changes due to progressive lowtemperature alteration of East Pacific Rise basalts from 0 to $9 \mathrm{Ma}$. Chemical Geology, 218: 281-313.

Schwertmann, U. and Cornell, R.M., 2000. Iron oxides in the laboratory: Preparation and characterization. WILEY-VCH, Weinheim, Germany, 188 pp.

Schwertmann, U., Fischer, W.R. and Papendorf, H., 1968. The influence of organic compounds on the formation of iron oxides. Trans. 9th Int. Congr. Soil Sci. Adelaide, 1: 645-655.

Seyfried Jr., W.E. and Bischoff, J.L., 1979. Low temperature basalt alteration by seawater: an experimental study at $70^{\circ} \mathrm{C}$ and $150^{\circ} \mathrm{C}$. Geochimica et Cosmochimica Acta, 43: 1937-1947. 
Seyfried Jr., W.E. and Bischoff, J.L., 1981. Experimental seawater-basalt interaction at $300^{\circ} \mathrm{C}, 500$ bars, chemical exchange, secondary mineral formation and implication for the transport of heavy metals. Geochimica et Cosmochimica Acta, 45(2): 135-147.

Staudigel, H. and Hart, S.R., 1983. Alteration of basaltic glass: Mechanisms and significance for the oceanic crust-seawater budget. Geochim. Cosmochim. Acta, 47: 337-350.

Staudigel, H., Hart, S.R. and Richardson, S.H., 1981. Alteration of the oceanic crust: Processes and timing. Earth Planet. Sci. Lett., 52(2): 311-327.

Stroncik, N.A. and Schmincke, H., 2001. Evolution of palagonite: Crystallization, chemical changes, and element budget. Geochemistry, Geophysics, Geosystems, 2.

Taylor, A.S. and Lasaga, A.C., 1999. The role of basalt weathering in the Sr isotope budget of the oceans. Chem. Geol., 161: 199-214.

Humphris, S. and Thompson, G., 1978. Hydrothermal alteration of oceanic basalts by seawater. Geochimica et Cosmochimica Acta, 42(1): 107-125.

Teo, B., 1986. EXAFS: Basic Principles and Data Analysis. Springer-Verlag.

Tebo, B.M. et al., 2004. Biogentic manganese oxides: properties and mechanisms of formation. Annu. Rev. Earth Planet. Sci., 32: 287-328.

Techer, I., Advocat, T., Lancelot, J. and Liotard, J.-M., 2001. Dissolution kinetics of basaltic glasses: control by solution chemistry and protective effect of the alteration film. Chem. Geol., 176: 235-263.

Templeton, A.S., Staudigel, H. and Tebo, B.M., 2005. Diverse Mn(II)-oxidizing bacteria isolated from submarine basalts at Loihi Seamount. Geomicrobiology Journal, 22: 127-139.

Trolard, F. et al., 1997. Identification of a green rust mineral in a reductive soil by Mossbauer and Raman spectroscopies. Geochim. Cosmochim. Acta, 61: 11071111.

Webb, S.M., 2005. SIXPACK: A graphical user interface for XAS analysis using IFEFFIT. Phys. Scr., T115: 1011-1014.

Wheat, C.G., Jannasch, H.W., Kastner, M., Plant, J.N. and DeCarlo, E.H., 2003. Seawater transport and reaction in upper oceanic basaltic basement: chemical data from continuous monitoring of sealed boreholes in a ridge flank environment. Earth and Planetary Science Letters, 6867: 1-16. 


\section{Chapter 6. Summary}

\subsection{Summary}

The ocean crust biosphere has garnered significant attention in recent years (Bach and Edwards, 2003; Banerjee and Muehlenbachs, 2003; Cowen et al., 2003; Edwards et al., 2005; Fisk et al., 1998; Furnes et al., 2001; Huber et al., 2006; Lysnes et al., 2004; Staudigel et al., 2004; Thorseth et al., 1995; Torsvik et al., 1998) as several studies present evidence suggesting that this rocky, crustal aquifer is not a barren landscape devoid of all life, but rather a productive environment with a dynamic microbial community. These findings are potentially of great consequence as the ocean crust covers more than $60 \%$ of the Earth's surface (possibly representing one of the most extensive endolithic communities on the planet) and an active biosphere has the potential to play a large role in the geochemical cycling of numerous elements in the crust and the deep-sea. Despite thermodynamic and bioenergetic calculations (Bach and Edwards, 2003) that estimate that the complete oxidation of $\mathrm{Fe}$ and $\mathrm{S}$ by chemolithoautotrophic microorganism in basaltic crust could support significant biomass production, very few studies (Cowen et al., 2003; Huber et al., 2003; Huber et al., 2006; Lysnes et al., 2004) have produced direct, indisputable evidence supporting this paradigm shift. Furthermore, no studies have yet been published regarding the activity and abundances of the communities in the ocean crust. The objectives of this thesis were to provide direct evidence regarding the types, distribution, abundances, and activities of microorganisms inhabiting this rocky substrate, and to further progress our understanding of the energy 
sources fueling this biosphere. Additionally, we set out to gain a better understanding of processes involved in the early stages of low-temperature basaltic glass alteration, which could be relevant for determining the effect of microbial activity on the alteration and evolution of the ocean crust.

In chapters 2 and 3, we examined the microbial communities existing on (and within) 5 geochemically different basaltic lavas collected from the seafloor at the East Pacific Rise (EPR), $9^{\circ} \mathrm{N}$. In chapter 2 we established the potential global importance of the seafloor ocean crust biosphere (in young, relatively fresh crust) with what was perhaps one of our most surprising results: The bacterial species richness, a measure of phylogenetic diversity based on 16S rRNA clone library studies, recovered from the seafloor lavas is not only greater than seawater and other surrounding deep-sea environments but is near that of a soil environment. This comparison is significant as soil environments are considered to harbor the greatest bacterial diversity on Earth (Torsvik et al., 1990). The observation of high species richness is paralleled by the observation of high biomass in young, seafloor lavas. Prokaryotic biomass estimates using quantitative PCR (qPCR) in very young ( $<3 \mathrm{kyrs}$ ), fresh laves recovered from the ridge axis show approximately $10^{6}$ cells $\mathrm{g}^{-1}$ basalt, and this microbial community is heavily dominated by Bacteria. These observations are depicted in Fig. 6.1 (Indicated by region A on the diagram). Lavas exposed for a longer period of time at the seafloor near the mid-ocean ridge axis, and thus showing signs of oxidative alteration, support a more dense community (dominated by Bacteria) that is $1-3$ orders of magnitude greater than observed in the fresh lavas (see Fig. 6, region B). Furthermore, CARD-FISH analyses not only 
confirm that bacterial communities are more abundant, but show that the majority of the total population is actively metabolizing on these basalt surfaces. Our biomass measurements are supported by thermodynamic and bioenergetic calculations (based on previous calculations by Bach and Edwards (2003)) that estimate approximately $10^{7}-10^{9}$ cells $\mathrm{g}^{-1}$ fresh basalt can be supported by basalt oxidation reactions. In comparison, deep seawater supports only $\sim 10^{4}$ cells $\mathrm{mL}^{-1}$ fluid (equivalent to $10^{4}$ cells $\mathrm{g}^{-1}$ fluid) of which the community is equally divided between Bacteria and Archaea (Karner et al., 2001) (Fig. 6.1, region G). The cumulative analyses provide evidence supporting the hypothesis that oxidative basalt alteration reactions are fueling the ocean crust biosphere.

Chapter 3 is a detailed examination of the phylogenetic types and abundances of Bacteria that inhabit increasingly altered basaltic lavas. We show that the species richness observed in Chapter 2 is attributed to taxa belonging to 17 different major taxonomic groups, and these taxa are widely distributed amongst numerous families and genera - rarely were multiple clones recovered from the samples assigned to the same taxa (based on a 3\% distance cut-off). In comparison, taxa belonging to only 3 major groups were recovered from the surround deep seawater. Additionally, phylogenetic relationships between the environmental clones and well-characterized, cultured organisms were detailed, and these analyses suggest that the geochemical cycling of $\mathrm{Mn}$, $\mathrm{S}, \mathrm{N}$, and $\mathrm{C}$ may be affected by microbial activity occurring within the basaltic lavas. Most importantly, we established a positive correlation between the extent of rock alteration and the abundances, diversity, and species richess of the bacterial communities. The summation of the results in Chapters 2 and 3 provides even more evidence that 
microbial communities recovered from this oligotrophic, bare-rock environment are directly linked to basalt alteration. Essentially, these results further demonstrate that the overall basalt-hosted community is likely underpinned by chemosynthetic microorganisms fueled by oxidative alteration reactions occurring on the basalt surfaces.

We have established that young, seafloor basaltic lavas host an incredibly diverse and active microbial community, however the lateral and vertical extent of this biosphere is not fully elucidated. A study by Huber et al. (2006) demonstrated the existence of a relatively small population $\left(\sim 10^{5}\right.$ cells $\mathrm{g}^{-1}$ fluid) of bacteria in warm, hydrothermal fluids emanating from the ocean crust in crust nearly $3.5 \mathrm{Ma}$ (as depicted in Fig. 6.1, region C). In Chapter 4, we used similar techniques as in our previous two chapters to examine the microbial communities in old ( $>10 \mathrm{Ma})$, subsurface ocean crust, most of which was formed millions of years ago at the East Pacific Rise. Extracting and amplifying DNA from these drill-core lavas proved to be non-trivial. In fact, DNA was successfully recovered from only 1 out of 35 total samples after trying numerous modifications of the techniques used to extract DNA from young lavas. Sequencing results of the DNA from the successfully amplified sample suggest that this sample was severely compromised from contamination during the sampling process. Additionally, tests to determine the efficiency and sensitivity of our DNA extraction and amplification protocols show that our methods are capable of detecting low quantities of biomass. Therefore we conclude that the subsurface biosphere in "old" ocean crust is substantially less relative to "young" seafloor crust and may be below the detection level of most molecular and microbiological techniques. These results are depicted in Fig. 6.1, regions D-F). 
Throughout these studies, the relationship between microorganisms and basalt alteration is emphasized. However, we cannot definitively distinguish if the microorganisms are directly contributing to the basalt alteration processes or if the microbial communities are controlled by redox gradients formed during abiotic basalt oxidation/hydration reactions, hydrothermal input, and/or secondary mineral precipitation. To identify the foundation for this relationship, a clear understanding of the basalt alteration processes, both with and without the presence of microorganisms, is required. In chapter 5 , we examined the alteration products in young seafloor lavas from the EPR to gain a better understanding of the early stages of basaltic glass alteration. Synchrotron-based, micro-scale XAS and XRF techniques were successful in identifying smectite and $\mathrm{Fe}$ oxide phases in the glass alteration layers that are typically difficult to characterize by most modern techniques. An amorphous gel-layer often observed in studies examining volcanic glasses was not clearly discerned, however. The reason for these differences, however, is not clearly understood and may be clarified with further examination using synchrotron-based XRD analyses and EMPA analyses.

The overall results of this study clearly establish that the ocean crust biosphere is a dynamic system that requires closer examination to ascertain the full potential and impact of this ecosystem on the environment. The contrast in our results for young versus old lavas strongly supports a paradigm shift in our thinking about the rates, mechanisms, and pathways of geochemical exchange between ocean crust and the deepsea. 


\subsection{Figures}

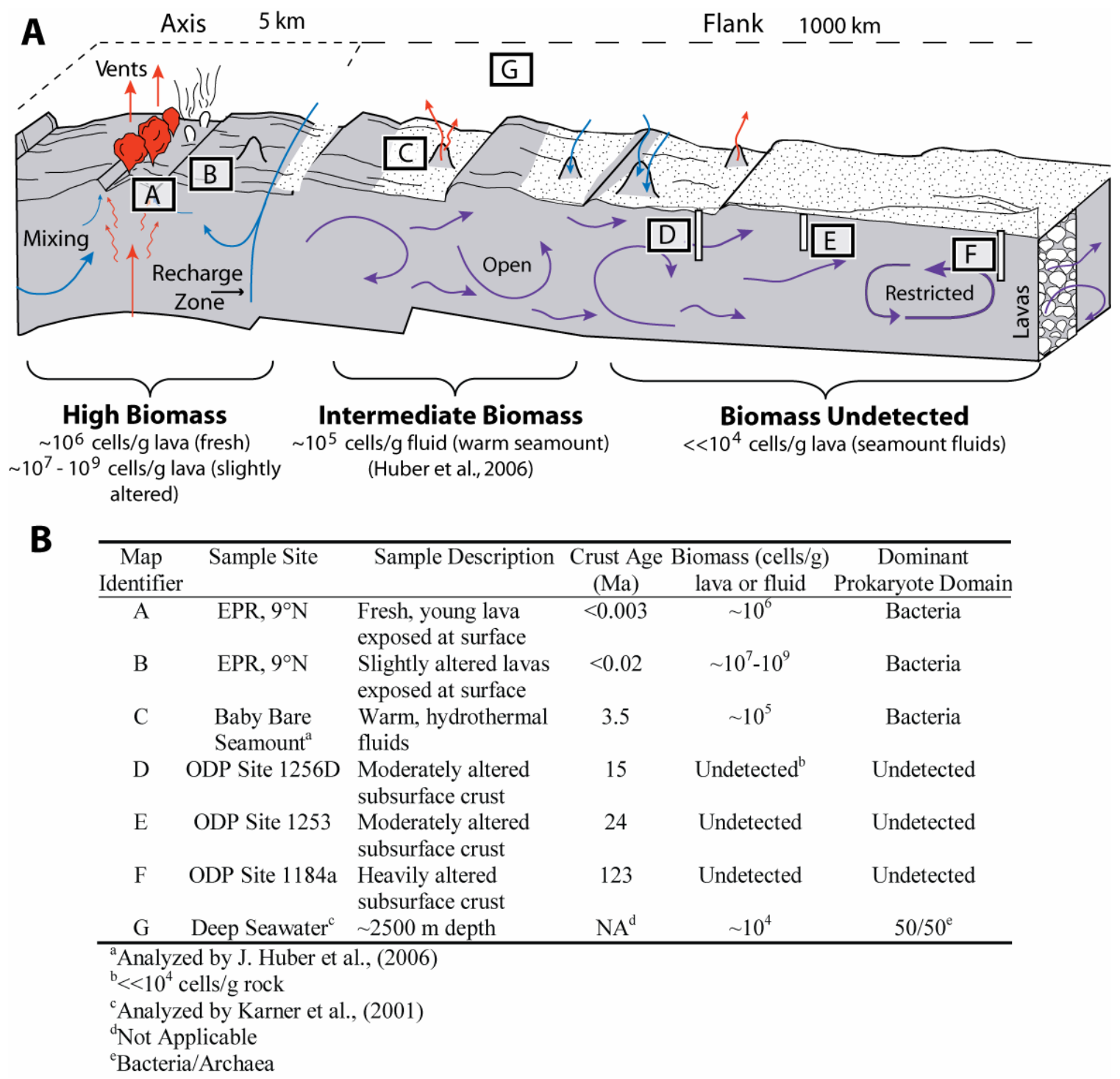

Figure 6.1. (A) Cross-sectional diagram of the upper portion (basaltic lava flows) of the ocean crust demonstrating the high prokaryotic biomass in young crust that generally decreases with increasing age of the crust (modified after Edwards et al., (2005)). Very young crust $(<20 \mathrm{kyrs})$, however, shows an increase in biomass during initial stages of basalt alteration. Microbial communities in old, subsurface crust could not be detected using standard, molecular biological tools. Letter indicators on map are described in table below. (B) Table describing map identifier (letters A-G). All analyses are from this study unless indicated. 


\subsection{References}

Bach, W. and Edwards, K.J., 2003. Iron and sulfide oxidation within the basaltic ocean crust: Implications for chemolithoautotrophic microbial biomass production. Geochim. Cosmochim. Acta, 67(20): 3871-3887.

Banerjee, N.R. and Muehlenbachs, K., 2003. Tuff life: Bioalteration in volcaniclastic rocks from the Ontong Java Plateau. Geochemistry Geophysics Geosystems, 4: Art. No. 1037(art. no. 1037).

Cowen, J.P. et al., 2003. Fluids from aging ocean crust that support microbial life. Science, 299(5603): 120-123.

Edwards, K.J., Bach, W. and McCollom, T.M., 2005. Geomicrobiology in oceanography: microbe-mineral interactions at and below the seafloor. TRENDS in Microbiology, 13(9).

Fisk, M.R., Giovannoni, S.J. and Thorseth, I.H., 1998. Alteration of Oceanic Volcanic Glass? Textural Evidence of Microbial Activity. Science, 281(978-980).

Furnes, H. et al., 2001. Bioalteration of basaltic glass in the oceanic crust. Geochemistry, Geophysics, Geosystems, 2: Art. No. 2000GC000150.

Huber, J., Butterfield, D. and Baross, J., 2003. Bacterial diversity in a subseafloor habitat following a deep-sea volcanic eruption. FEMS Microbiol. Ecol., 43(3): 393-409.

Huber, J.A., Johnson, H.P., Butterfield, D.A. and Baross, J.A., 2006. Microbial life in ridge flank crustal fluids. Appl. Environ. Microbiol., 8(1): 88-99.

Karner, M.B., DeLong, E.F. and Karl, D.M., 2001. Archaeal dominance in the mesopelagic zone of the Pacific Ocean. Nature, 409: 507-510.

Lysnes, K. et al., 2004. Microbial community diversity in seafloor basalt from the Arctic spreading ridges. FEMS Microbiology Ecology, 50: 213-230.

Staudigel, H. et al., 2004. The ocean crust as a bioreactor. In: W.S.D. Wilcock, E.F. DeLong, D.S. Kelley, J.A. Baross and S.C. Cary (Editors), The Subseafloor Biosphere at Mid-Ocean Ridges. American Geophysical Union, pp. 325-341.

Thorseth, I.H., Torsvik, T., Furnes, H. and Muehlenbachs, K., 1995. Microbes play an important role in the alteration of oceanic crust. Chemical Geology, 126: 137-146.

Torsvik, T., Hurnes, H., Muehlenbachs, K., Thorseth, I.H. and Tumyr, O., 1998.

Evidence for microbial activity at the glass-alteration interface in oceanic basalts. Earth and Planetary Science Letters, 162: 165-176.

Torsvik, V., Goksoyr, J. and Daae, F.L., 1990. High diversity in DNA of soil bacteria. Appl. Environ. Microbiol., 65(3): 782-787. 